University of Louisville

ThinkIR: The University of Louisville's Institutional Repository

Electronic Theses and Dissertations

$12-2005$

\title{
Novel processes for large area gallium nitride single crystal and nanowire growth.
}

Hongwei Li 1975-

University of Louisville

Follow this and additional works at: https://ir.library.louisville.edu/etd

\section{Recommended Citation}

Li, Hongwei 1975-, "Novel processes for large area gallium nitride single crystal and nanowire growth." (2005). Electronic Theses and Dissertations. Paper 825.

https://doi.org/10.18297/etd/825

This Doctoral Dissertation is brought to you for free and open access by ThinkIR: The University of Louisville's Institutional Repository. It has been accepted for inclusion in Electronic Theses and Dissertations by an authorized administrator of ThinkIR: The University of Louisville's Institutional Repository. This title appears here courtesy of the author, who has retained all other copyrights. For more information, please contact thinkir@louisville.edu. 
NOVEL PROCESSES FOR LARGE AREA GALLIUM NITRIDE SINGLE CRYSTAL AND NANOWIRE GROWTH

\author{
By
}

Hongwei Li

B. S., Shandong University of Technology, Jinan, China, 1997

M. S., Beijing University of Chemical Technology, Beijing, China, 2000

\author{
A Dissertation \\ Submitted to the Faculty of the \\ Graduate School of the University of Louisville \\ in Partial Fulfillment of the Requirements \\ for the Degree of
}

Doctor of Philosophy

Department of Chemical Engineering
University of Louisville
Louisville, KY, 40292

December 2005 

NOVEL PROCESSES FOR LARGE AREA GALLIUM NITRIDE SINGLE CRYSTAL AND NANOWIRE GROWTH

$$
\text { By }
$$

Hongwei Li

B. S., Shandong University of Technology, Jinan, China, 1997

M. S., Beijing University of Chemical Technology, Beijing, China, 2000

A Dissertation Approved on

August 26, 2005

by the following Dissertation Committee:

Prof. Mahendra K. Sunkara (Dissertation Director)

\begin{tabular}{c}
\hline Prof. Thomas L. Starr \\
\hline Prof. Raul Miranda \\
\hline Prof. Kevin M. Walsh \\
\hline Prof. Shi-yu Wu
\end{tabular}

Prof. Gamini Sumanasekera 


\section{DEDICATION}

This dissertation is dedicated to

my father Huaichun Li

and

my mother Shixiu Wang

who have never stopped supporting me for my education and every progress. 


\section{ACKNOWLEDGEMENTS}

I gratefully acknowledge all those people who help me through my studies and make the dissertation possible. My sincere appreciation first goes to my thesis advisor Prof. Mahendra K. Sunkara for his seasoned direction, continuous encouragement and support. I also greatly appreciate my thesis committee members Prof. Thomas Starr, Prof. Raul Miranda, Prof. Kevin Walsh, Prof. Shi-yu Wu, Prof. Gamini Sumanasekera for taking time to review this work and giving valuable suggestions. I also want to thank my colleagues, those who have graduated and those who will be graduating, Dr. Shashank Sharma, Dr. Radhika Mani, Dr. Gopinath Bhimarasetti, Mr. Hari Chandrasekaran and Mr. Sreeram Vaddiraju for their valuable help and keeping me energetic during my studies. Helpful discussions for the GaN crystal growth studies with Dr. Roy Gat at Coating Technology Solutions Inc., MA are also appreciated.

I also acknowledge my collaborators Prof. K. Rajan and Mr. M. Stukowski at Rensselaer Polytechnic Institute for help on TEM studies, Prof. Z. Sitar and Dr. R. Collazo at North Carolina State University for assistance with XRD texture analysis, Prof. J. Chaudhuri at Wichita State University for the synchrotron X-ray topography studies, Dr. S. I. Borenstain at El-Mul Technologies, Ltd., Israel for the cathodoluminescence characterization, Dr. W. Xu at University of Kentucky for help on HRTEM characterization and Dr. A. Chin at NASA Ames Nanotechnology Research Center for photoluminescence analysis. Financial supports from Army research, Air Force Office of 
Scientific Research (AFOSR), Kentucky Science \& Technology Council (KSTC) and National Science Foundation (NSF) are greatly appreciated.

I would like to express my grateful acknowledgement to my wife Ms. Jiru Xie for accompanying me in U.S. where I am far away from my parents and relatives. Her constant support and encouragement make the studies possible.

Finally my sincere thanks go to my parents, my father Huaichun Li and my mother Shixiu Wang for making all this possible with their endless supports. I owe them too much and no words could express my gratitude.

I was born in a small, typical rural village in China. I couldn't remember but I could imagine the hardness my parents experienced at that time when people were still struggling for adequate food and clothing. I survived from twice serious drown when I was 2-3 years old, but resulted in serious cough from the accidents. My parents worried about me, spent every penny they had to try every recipe they could get to cure the disease. Thanks to my parents' carefully and meticulously effort, I finally recovered and grow strong enough to be here to achieve the dream.

My father could have had gone to college after high school if there were no the culture revolutionary. My mother only had finished primary school and had to quit to support her family. Though not well educated, they know the importance of education for their children's future. With only about 1 acre land and almost everything being done by hands, they planted, irrigated, harvested the crops to feed the family, though most time the harvest has to be under the mercy of weather. They worked as long as 16 hours a day, sometimes even in the sweltering summer to try their best to give us a better living. Because of very less profits from only growing wheat, my father also grew vegetables to 
sell them on the market and did many kinds of small businesses to subsidize the family. I tried to help them by doing things as much as I could since I was in primary school. I still remember those times of hard labor works: I often got up early morning to help crop the vegetables for sale; I was waked up from sweet dream at midnight to pick up the sweet potato being dried on the land before the unexpected rain came; I helped plant the wheat with chilly water in the mid fall morning before sun rising; We cracked the peanuts by hands during the winter nights till the hands ached. I understand the arduousness my parents had gone through by experiencing these hard labor works. All these experiences provide me with valuable assets of hard working, frugality and always stimulate me to move forward.

My parents are always proud of me and never stop supporting my pursuit. Without them, I would never make this journey. Living far away from my parents, my home country, I want share my every success with them and this dissertation is my best present for them! 


\section{ABSTRACT \\ NOVEL PROCESSES FOR LARGE AREA GALLIUM NITRIDE SINGLE CRYSTAL AND NANOWIRE GROWTH}

Hongwei Li

August 26, 2005

III-nitrides (InN, GaN, AlN) are some of the most promising materials for making blue light emitting diodes (LED), blue laser diodes (LD) and high power, high temperature field effect transistors (FET). Current techniques produce GaN films with defect densities on the order of $10^{7} / \mathrm{cm}^{2}$ or higher. The performance and life-time of the devices critically depend upon the defect densities and high power, high frequency devices require the defect densities to be lower than $10^{4} / \mathrm{cm}^{2}$. So, the need for new processes to produce large size GaN crystals with defect densities less than $10^{7} / \mathrm{cm}^{2}$ is immediate. In addition to large area single crystals (or wafers), the nanowires also present as an alternating platform for making devices. So, the processes for controlled synthesis, modifying and integrating sub $100 \mathrm{~nm}$ nanowires into electronic devices are of great interest.

This thesis presents a new concept of 'self-oriented growth' of GaN platelets shaped crystals on molten gallium to produce near single crystal quality GaN films over large areas $\left(>1 \mathrm{~cm}^{2}\right)$. The process involves direct nitridation of Ga films using nitrogen plasma at low pressures (few mTorr). GaN flakes with areas over $25 \mathrm{~mm}^{2}$ have been successfully obtained. Raman spectra of the resulting GaN crystals show no stress and 
low native donor concentration on the order of $10^{17} / \mathrm{cm}^{3}$. XRD texture analysis showed an overall c-axis tilt of $2.2^{\circ}$ between $\mathrm{GaN}$ domains within the flake. The cross-sectional TEM micrographs showed that the resulting GaN films are free from dislocation crops inside the grains but showed diffraction contrast due to small mis-orientation between the grains. The twist and tilt angles between adjacent columnar grains were determined using convergent beam electron diffraction technique to be less than $8^{\circ}$ and $1^{\circ}$, respectively. HRTEM micrographs of the grain boundaries showed sharp interfaces resulting with both twisted and perfect attachments.

This thesis also presents direct synthesis approach for $\mathrm{GaN}$ nanowires with control on growth directions using modified nitridation conditions. The nitridation in the presence of hydrogen or ammonia resulted in oxide sheath free GaN nanowires as thin as $20 \mathrm{~nm}$ and long as $100 \mu \mathrm{m}$ in $<0001>$ direction. The nitridation using low Ga flux in a vapor transport set-up resulted in sub $100 \mathrm{~nm} \mathrm{GaN} \mathrm{nanowires} \mathrm{with}<10-10>$ growth direction. The difference in the nucleation and growth mechanism allowed control on the nanowire directions. Homo-epitaxial experiments onto pre-synthesized GaN nanowires with the above two growth directions using the vapor transport of $\mathrm{Ga}$ and dissociated ammonia exhibited different morphologies, e.g. micro hexagonal columns for $<0001>$ nanowires and micro belts for nanowires with $<10-10>$ growth direction. The results further illustrate a new phenomenon of enhanced surface diffusion on nanowires in general but more pronounced for wires with $<0001>$ growth direction. The results from homo-epitaxy experiments suggest that the $<10-10>$ direction wires could be used as "seeds" for growing large area GaN crystals in vapor phase homo-epitaxy schemes. 
TABLE OF CONTENTS

PAGE

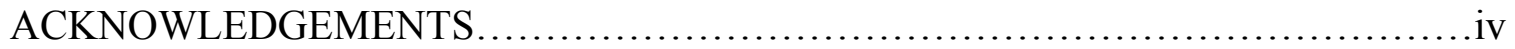

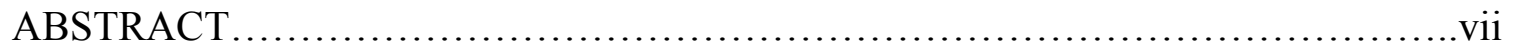

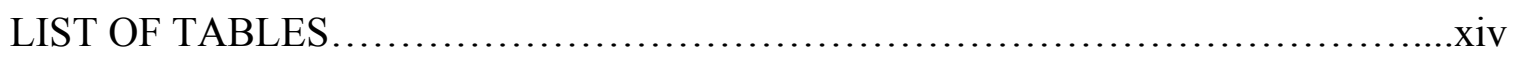

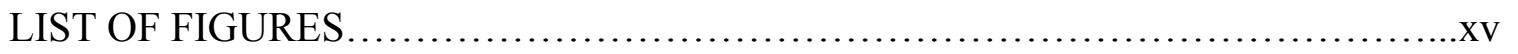

CHAPTER

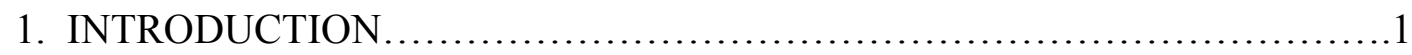

2. BACKGROUND.....................................................

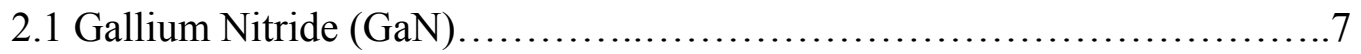

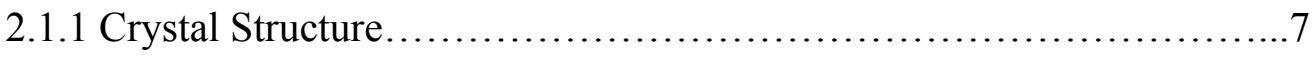

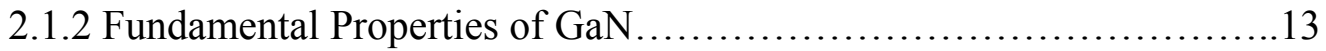

2.1.3 Applications................................................14

2.2 State-of-the-art GaN Crystal Growth.................................16

2.2.1 Epitaxial Growth............................................ 16

2.2.1.1 Metal Organic Chemical Vapor Deposition (MOCVD).....17

2.2.1.2 Molecular Beam Epitaxy (MBE).......................18

2.2.1.3 Hydride Vapor Phase Epitaxy (HVPE)...................20

2.2.1.4 Defects Engineering Techniques......................23

2.2.2 Road Map of GaN Crystal Growth...............................25

2.2.3 Bulk Single Crystal Growth.....................................26

2.2.3.1 High Pressure Solution Growth.........................26 
2.2.3.3 Ammonothermal Growth....................................29

2.2.3.4 Low Pressure Approach....................................30

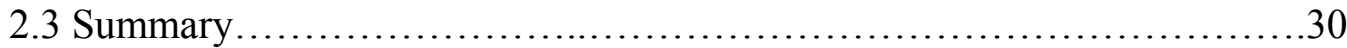

\section{PROPOSED CONCEPTS FOR GALLIUM NITRIDE CRYSTAL GROWTH}

AND NANOWIRE SYNTHESIS …......................................

3.1 GaN Formation Using Nitrogen Plasma at Sub-atmospheric Pressures .....31

3.2 Proposed Concept of Self-oriented GaN Growth on Molten Gallium........32

3.3 Proposed Concept for GaN Nanowires Synthesis with Control on Growth

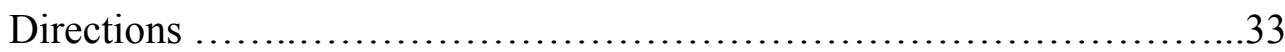

3.4 Proposed Concept of Homo-epitaxy onto Long GaN Nanowires for Large

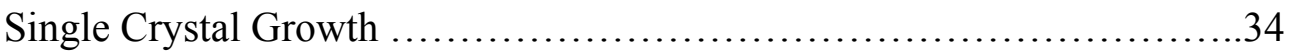

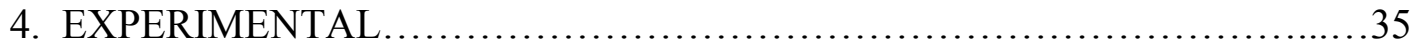

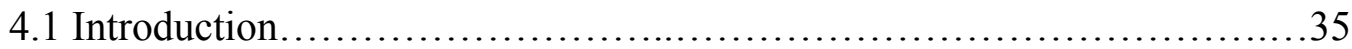

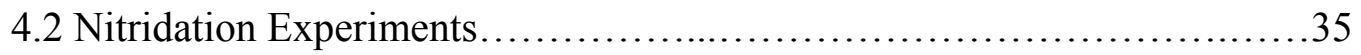

4.2.1 ECR-MW Plasma Reactor.............................................35

4.2.2 Substrate Heater Set-ups..............................................

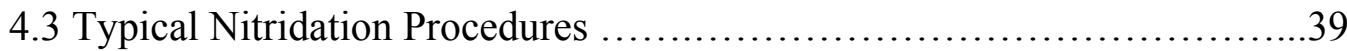

4.4 Materials Characterization Techniques..................................40

4.4.1 X-ray Diffraction................................................ 40

4.4.1.1 Single Crystal Diffraction.................................41

4.4.1.2 Texture Analysis.........................................42

4.4.2 Transmission Electron Microscopy................................44 
4.4.2.1 Convergent Beam Electron Diffraction (CBED)................44

4.4.2.2 Kikuchi Lines..............................................45

4.4.2.3 Scanning Transmission Electron Microscopy (STEM)............46

4.4.2.4 Sample Preparation......................................47

4.4.3 Raman Spectroscopy..........................................48

4.4.4 Luminescence...............................................48

4.4.4.1 Photoluminescence.....................................48

4.4.4.2 Cathodoluminescence................................49

4.4.4.3 Time-resolved Cathodoluminescence........................49

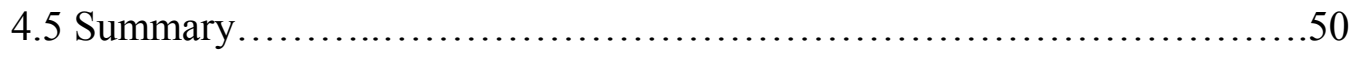

5. ORIENTED GALLIUM NITRIDE GROWTH ON MOLTEN GALLIUM......51

5.1 Introduction..........................................................

5.2 Dynamic Growth Processes of GaN from Molten Ga....................51

5.2.1 Self-spreading Phenomena of Nitrogenated Ga......................51

5.2 .1 .1 Wetting................................................ 51

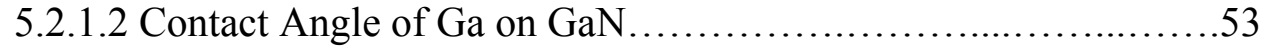

5.2.1.3 Flow of Molten Ga.........................................55

5.2.2 Mechanistic Studies of GaN Crystal Growth........................57

5.2.2.1 Morphologies of GaN Crystals.............................58

5.2.2.1.1 Wulff Theory of Crystal Morphology Applied to GaN Crystal Growth......................................58

5.2.2.1.2 Unusual Morphologies of GaN Crystals....................58

5.2.2.2 Sequential Growth of Self-oriented GaN Film..................65 
5.3 Large Area Self-oriented GaN Film Growth on Molten Ga. 67

5.3.1 Self-oriented GaN on molten Ga.................................67

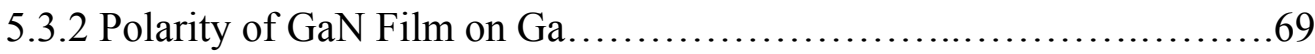

5.3.3 Stresses and Native Carrier Concentration of GaN Grown on Ga........71

5.3.4 Orientation of GaN Film on Molten Ga.............................76

5.3.4.1 XRD Texture Analysis over Large Area Film....................76

5.3.4.2 TEM Studies of Orientation between Individual Crystals...........78

5.3.4.2.1 Cross Sectional TEM Analysis..............................79

5.3.4.2.2 Kikuchi Line Analysis....................................... 80

5.3.4.2.3 High Resolution TEM of Grain Boundaries.................81

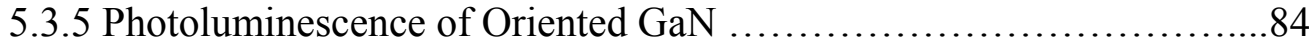

5.3.6 Large Area Self-Oriented Film.........................................86

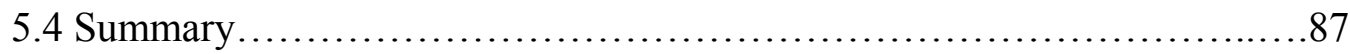

6. DIRECT SYNTHESIS OF GALLIUM NITRIDE NANOWIRES WITH

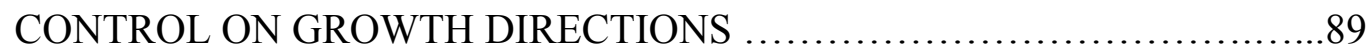

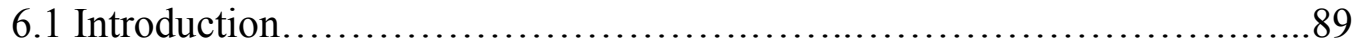

6.2 Experimental ...................................................... 91

6.3 Result and Discussion .............................................. 93

6.3.1 Growth of $<0001>\mathrm{GaN}$ nanowires..............................93

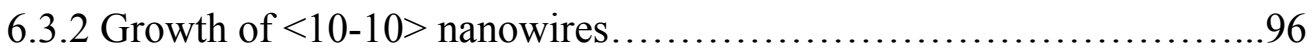

6.3.3 Optical Properties of GaN Nanowires..............................99

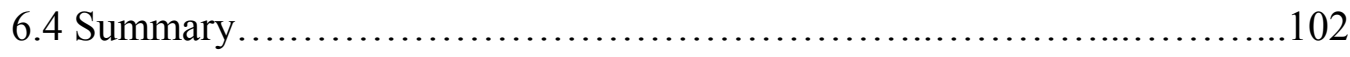

7. HOMO-EPITAXIAL GROWTH OF GALLIUM NITRIDE NANOWIRES....103 
7.1 Introduction................................................... 103

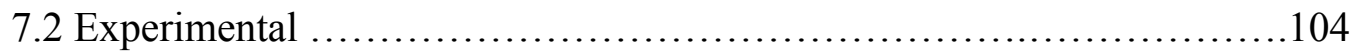

7.3 Result and Discussion......................................... 105

7.3.1 Homo-epitaxial Growth onto $<0001>$ Nanowires.................... 105

7.3.2 Homo-epitaxial Growth onto $<10-10>$ Nanowires.....................107

7.3.3 Kinetic Growth of GaN Nanowires.............................110

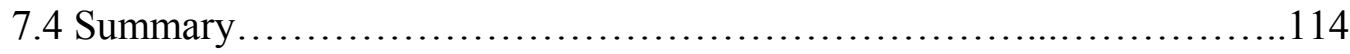

8. CONCLUSIONS ....................................................... 115

9. PERSPECTVIES ................................................ 118

9.1 Large Area GaN Crystal Growth Using Rotating Substrate Stage.........118

9.2 Electronic Properties of GaN nanowires..............................119

9.3 III-nitrides Nanoscale Heterostructures.............................121

REFERENCES........................................................ 125

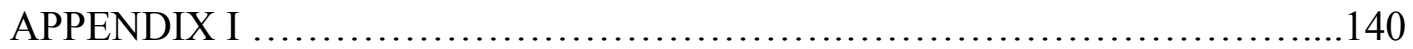

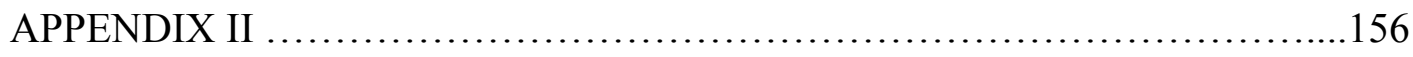

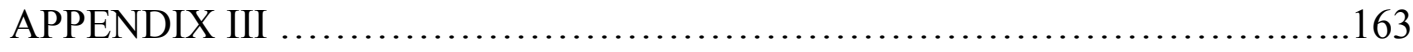

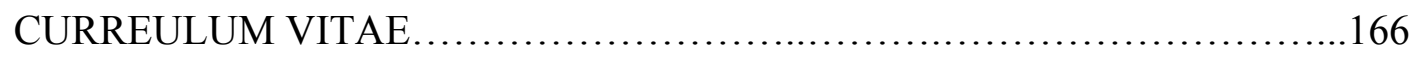




\section{LIST OF TABLES}

TABLE

PAGE

1.1 Comparison of properties of several common semiconductor materials..............

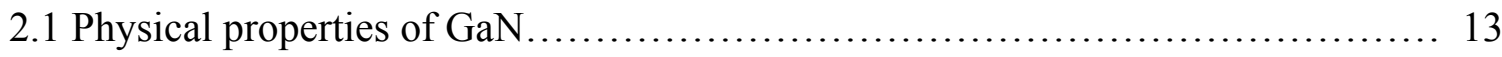

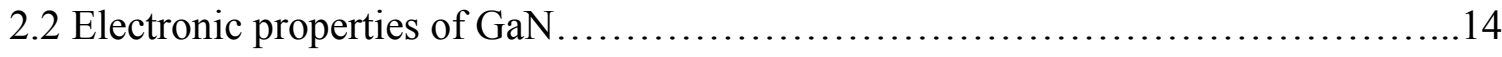

2.3 Comparisons of the three $\mathrm{GaN}$ vapor phase epitaxy growth techniques............22

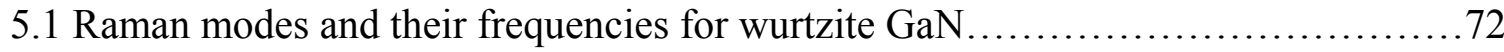

A1.1 Rocking curve of 4 different GaN samples.............................. 145

A3.1 Properties of gallium................................................ 164 


\section{LIST OF FIGURES}

\section{FIGURE}

PAGE

1.1 The lattice constant and band gap of common semiconductor materials.............2

2.1 Structure of HCP lattice.................................................... 8

2.2 Symmetry elements of point group $6 \mathrm{~mm}$ for hexagonal $\mathrm{GaN} \ldots \ldots \ldots \ldots \ldots \ldots \ldots . \ldots . \ldots$

2.3 3-D view of crystallographic structure of hexagonal GaN...................... 10

2.4 Comparison between HCP and FCC structures................................. 11

2.5 The symmetry elements of the FCC structure of cubic GaN......................11

2.6 3-D view of crystallographic structure of cubic GaN..........................12

2.7 Typical chambers for MBE GaN growth..................................... 19

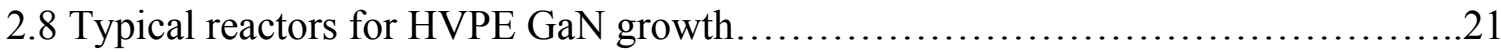

2.9 Sketch of the LEO process for GaN growth................................24

2.10 Road map showing the need for bulk growth techniques for defects reduction.....26

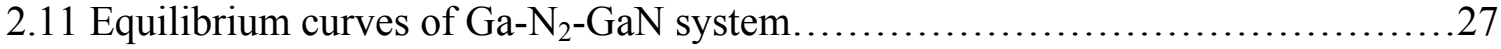

3.1 Sketch of self-oriented growth of GaN platelets on molten gallium to form large area

GaN crystal........................................................... 33

4.1 Sketches of side-view and top-view of the ECR reactor with stationary heater.......36

4.2 Sketch of ECR-MW system........................................... 37 
4.3 Pyrolytic boron nitride (pBN) heating set-up; (a) Ceramic heater assembly (b) Heater

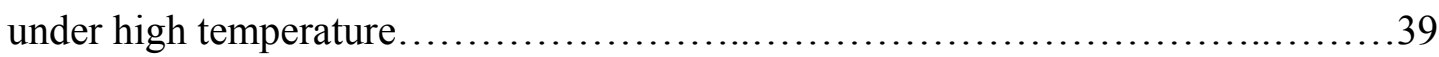

4.4 Configuration of powder XRD setup ....................................41

4.5 X-ray texture diffractometer for determining the orientation of large area oriented GaN crystals........................................................ 43

4.6 (a) Convergent beam mode (b) Sketch of ray diagram for Kikuchi lines in TEM....45

5.1 Wetting of a liquid on a solid surface....

.52

5.2 Spreading of Ga over substrates: (a) Optical photograph illustrating the spreading phenomenon of gallium over quartz substrates (b) Cross sectional SEM micrograph of the region near the original Ga shows the GaN formation and subsequent Ga film spreading onto it .56

5.3 SEM photographs illustrating wetting of $\mathrm{Ga}$ on $\mathrm{GaN}$ during nitridation of Ga droplets using $\mathrm{NH}_{3}$

5.4 (a) GaN platelets-shaped crystals (b) Standing hollow platelets crystals .59

5.5 Standing GaN hollow platelets with struts between two layers indicating growth instability due to convection of gallium.

5.6 GaN crystals with variable depressions on top surfaces.... .61

5.7 Various star-shaped GaN crystals

5.8 The star morphologies with 6-fold symmetry from a hexagon: (a) \& (d) The two basic kinds of stars with 6-fold symmetry from a hexagon; (b, c, e, f) Four different kinds of six-fold symmetrical stars bound by low index planes $(\leq 3)$ .64 
5.9 Side views of the star-shaped GaN crystals .64

5.10 Plan view SEM images of sequential growth of oriented $\mathrm{GaN}$ on top of gallium....65

5.11 Cross sectional SEM micrographs of sequential growth of oriented GaN on top of gallium .66

5.12 Representative SEM images of free-standing, c-plane oriented GaN films after Ga was dissolved away: (a) plan view and (b) cross section view. Inset in (b) shows SEM image of a cross sectional TEM sample after polishing .68

5.13 XRD spectra of GaN films obtained under two different wetting conditions of molten Ga. (a) Nitridation experiment when Ga melt spreads and (b) GaN crust on Ga when Ga does not spread 68

5.14 The SEM images of two different GaN films: (a) GaN grown directly on top of Ga and (b) GaN grown directly on the amorphous Quartz substrate without the Ga buffer layer.

5.15 SEM images of GaN film with $\mathrm{KOH}$ etching for polarity determination. (a) Top surface before etching; (b) Top surface after etching; (c) Bottom surface before etching; (d) Bottom surface after etching. .71

5.16 Raman spectra of oriented GaN film with laser beam parallel and perpendicular to the c-axis 73

$5.17 \mathrm{E}_{2}^{2}$ phonon frequencies of GaN films grown directly on molten $\mathrm{Ga}$; amorphous quartz; single crystalline sapphire. The results indicate that GaN grown on molten $\mathrm{Ga}$ is stress-free. 
5.18 Profile of LO-phonon-plasmon coupled modes for GaN films grown directly on molten $\mathrm{Ga}$ and on amorphous quartz. The results indicates low native carrier concentration with GaN film grown directly on molten Ga......................76

5.19 (a) XRD texture analysis of a $5 \mathrm{~mm} \times 5 \mathrm{~mm}$ free standing GaN flake; (b) SEM image showing the presence of skewed crystals in certain regions within the film which cause the presence of (10-11) reflection in the plot showing in (a); (c) $\chi$ integration of the scan indicates preferential c-plane orientation; (d) $2 \theta$ integration of the (0002) reflection line showing a FWHM of $2.2^{\circ} \ldots \ldots \ldots \ldots \ldots \ldots \ldots \ldots \ldots . . . \ldots . \ldots \ldots$

5.20 (a) TEM bright field image of a cross-section of GaN film with $2^{\text {nd }}$ grain on ZA $<11-20>$ showing contrast resulting from misorientations of few grains. (b) Selected

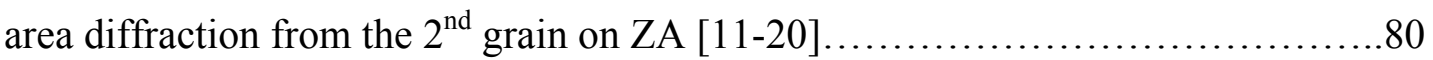

5.21 Experimental and simulated kinematical Kikuchi lines using CBED patterns from four grains with the following misorientation: (1) on ZA [11-20]; (2) $8^{\circ}$ twist, $0.2^{\circ}$ tilt (3) $3^{\circ}$ twist, $-0.4^{\circ}$ tilt; (4) $9.7^{\circ}$ twist, $0.1^{\circ}$ tilt; with respect to grain $1 \ldots \ldots \ldots \ldots \ldots . .81$

5.22 (a) A boundary with no misorientation. Insets show the corresponding CBED patterns for the two grains forming the boundary; (b) HRTEM image of the boundary shows the intermittent disordered layer at the boundary (c) HRTEM images of the bottom part of the boundary shows no disordered layer.

5.23 (a) STEM image of the boundary, the line indicates the region for EDX line scan;

(b) $\mathrm{N}$ concentration profile along the boundary shown in (a), dwell time $15 \mathrm{~s}$, resolution $0.5 \mathrm{~nm}$ .83

5.24 (a) HRTEM images of the straight boundary between grains 2 and 3 shown in Fig. 5.20 with certain a misorientation of $5^{\circ}$ twist in c-plane and $0.6^{\circ} \mathrm{c}$-axis tilt; (b) 
HRTEM images of the (1-101) faceted boundary between grains 3 and 4 shown in

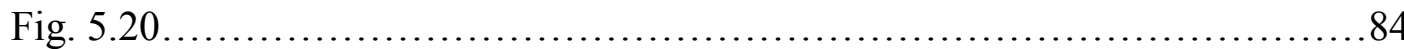

5.25 UV photoluminescence of GaN grown on Ga and on amorphous quartz substrates showing defects related luminescence for $\mathrm{GaN}$ directly grown on quartz..........85

5.26 Visible and UV PL of GaN on Ga showing yellow luminescence centered at $2.2 \mathrm{eV}$ which might originates from $\mathrm{N}$ vacancies at the grain boundaries................86

5.27 Optical images and XRD spectra of large area oriented flakes....................87

6.1 Experimental setup for (a) $<0001>\mathrm{GaN}$ nanowire synthesis from Ga droplets; (b) reactive vapor transport growth of $<10-10>\mathrm{GaN}$ nanowire in $\mathrm{NH}_{3}$ atmosphere....93

6.2 (a) Ga droplets with considerable size distribution on quartz substrates (b) GaN wires on top of gallium droplets $\mathrm{H}_{2}$ and $\mathrm{N}_{2}$ plasma.............................94

6.3 High density of GaN nanowire as thin as $15 \mathrm{~nm}$, up to hundred microns long from direct reaction between $\mathrm{Ga}$ and $\mathrm{NH}_{3}$ .94

6.4 (a) TEM micrographs of $<0001>$ GaN nanowires, inset is a $27 \mathrm{~nm}$ nanowires with stacking faults in c-plane along the wire; (b) HRTEM of GaN showing growth direction of $<0001>$ and no oxide sheath, inset is the FFT pattern with ZA

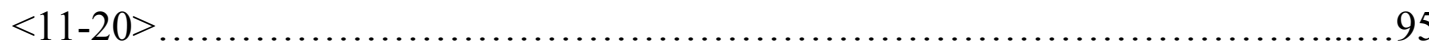

6.5 Sketch of $\mathrm{GaN}<0001>$ nanowires growth with basal growth from Ga melts........96

6.6 (a) $<10-10>\mathrm{GaN}$ nanowires with average diameter of $25 \mathrm{~nm}$ using reactive vapor transport of $\mathrm{Ga}$ in the presence of $\mathrm{NH}_{3}$; (b) TEM micrographs of $<10-10>$ nanowires with no defects and oxide sheath

6.7 Sketch of $<10-10>\mathrm{GaN}$ nanowires growth from the GaN crystals through liquid phase epitaxy 
$6.8 \mathrm{GaN}<10-10>$ nanowires grown from side facets of $\mathrm{GaN}$ hexagonal platelets using reactive vapor transport in the presence of $\mathrm{NH}_{3}$

6.9 UV-PL of (a) $<0001>$ and (b) $<10-10>\mathrm{GaN}$ nanowires with different power density showing red-shift with increasing laser power indicate laser heating effect on the GaN nanowires

6.10 (a) UV PL of $<0001>\mathrm{GaN}$ nanowires with different laser power fraction after correction assuming a Gaussian shape for the spectral dip (b) Bandgap of GaN nanowires with $<10-10>$ direction shows blue-shift about $50 \mathrm{meV}$ compared with $<0001>$ GaN nanowires.....................................................

7.1 Homo-epitaxial experiments onto GaN nanowire in $\mathrm{NH}_{3}$ atmosphere

7.2 SEM images showing the resulting morphologies with homo-epitaxial growth onto $<0001>\mathrm{GaN}$ nanowires

7.3 (a) Low magnification TEM images of homo-grown GaN hexagonal prisms from $<0001>$ nanowires; (b) HRTEM micrograph of the nanowires showing stacking faults in c-plane.

7.4 (a) SEM images showing the resulting micro-belt morphologies obtained with homoepitaxial growth onto $<10-10>$ nanowires. (b) A $900 \mathrm{~nm}$ microbelt with preferential growth from one edge of the $<10-10>$ nanowire 108

7.5 Preferential growth of microbelts from $<10-10>\mathrm{GaN}$ nanowires with vapor transport growth in presence of $\mathrm{NH}_{3}$ in some cases 109

7.6 (a) TEM micrographs of a homo-epitaxial grown $100 \mathrm{~nm}$ micro belts from $<10-10>$ GaN nanowire showing Ga terminated and N-terminated surfaces; (b) SAD pattern 
with zone axis $(\mathrm{ZA})<11-20>$ and high resolution TEM indicating that the direction

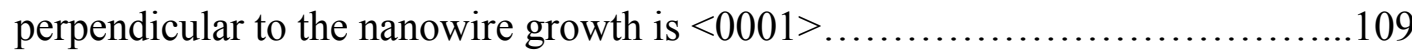

7.7 Raman spectrum of the GaN microbelts showing only $E_{1}(\mathrm{TO})$ peak at $561 \mathrm{~cm}^{-1}$ indicating the large surface of the microbelts is perpendicular to the c-axis.......110

7.8 (a) Growth steps on $\{10-11\}$ pyramidal side facets indicating growth from nanowires onto hexagonal prisms. (b) Schemes of 1-D diffusion growth processes forming hexagonal islands

7.9 Sketch of homo-epitaxial growth processes for homo-epitaxial growth onto $<0001>$ GaN nanowires

7.10 (a) $\{10-10\}$ oriented GaN crystal grown from $\mathrm{GaN}<10-10>$ nanowires (b) Schemes of microbelts formation from $<10-10>$ nanowires

9.1 ECR-MW reactor with rotating substrates stage for controlling the flow of molten gallium for large area oriented $\mathrm{GaN}$ synthesis

9.2 (a) Schematic of a nanowire FET and (b) FE-SEM image of FET made out of GaN nanowires for electronic properties measurement.

9.3 (a) Evaporated In onto GaN nanowires (b) GaN-InN core-shell heterostructures...122

9.4 (a) GaN core-AlN sheath nanostructure; (b) GaN branched nanostructure...........123

9.5 Aligned III-nitride nanostructures...................................... 123

A1.1 Plan view and cross sectional view of two different zones of GaN on sapphire substrates. (a) GaN on $\mathrm{Ga}$ (b) GaN directly on sapphire.

A1.2 Growing and coalescing of GaN crystals on Ga with sapphire substrates.... 142

A1.3 XRD spectra of $\mathrm{GaN}$ with sapphire substrates indicating C-plane orientation 
A1.4 (a) Synchrotron white beam x-ray transmission Laue diffraction pattern from $\mathrm{GaN}$ film on sapphire, exposure time $10 \mathrm{sec}$ and specimen to film distance $10 \mathrm{~cm}$. (b) Enlarged one of the diffraction spot in (b), $g=24 . \overline{2}$, wavelength $=0.2657 \AA, A=$ cellular structure of GaN crystals. (c) Simulated Laue DP of GaN, (0001) face, 50 $\mathrm{KeV}$, film distance $=10 \mathrm{~cm}$. (d) Simulated Laue DP of sapphire, (0001) face, 50

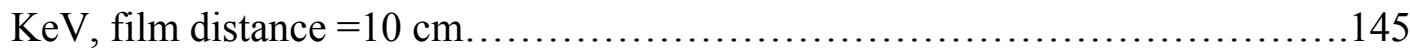

A1.5 Raman spectrum of $\mathrm{GaN}$ on sapphire in back scattering configuration indicates wurtzite phase. The two broad bumps on the right side are from the sapphire substrates. ....

A1.6 Photon counting setup for time-resolved CL measurement. HV-High Vacuum, LGLight guide, MCP-micro-channel plate, PMT-photo multiplier. 148

A1.7 The CL intensity map of the GaN samples with UV filters at room temperature. (a) UV photoluminescence dominating sample (b) Yellow luminescence dominating sample. The bar on the right side denotes the PMT current. .150

A1.8 Normalized CL spectra of GaN from different regions shown in Fig. A1.7; (1) and (2): with dominating near band edge emission; (3) with dominating yellow luminescence. 150

A1.9 Response time of GaN on sapphire with dominating UV emission measured with and without UV filter at room temperature, the line is the fitted curve of data....152

A2.1 (a) Optical micrograph of spun coated gallium film on 2-inch quartz substrate. (b) Optical micrograph of the as-nitrided substrate. (c) SEM image of nano-crystalline GaN film showing crystallite size of $85 \mathrm{~nm}$. .157 
A2.2 XRD of textured nanocrystalline GaN showing dominate (0002) peak of wurtzite phase. 158

A2.3 Cross sectional TEM images of nanocrystalline GaN film on quartz substrate showing sharp interface of amorphous quartz and GaN.......................158

A2.4 Cross section TEM micrographs of nanocrystalline GaN with regrowth show increased grain size (a) one cycle of growth (b) four cycles of growth.............159

A2.5 UV-Vis spectra of nanocrystalline GaN on amorphous quartz....................161

A2.6 Tauc plot of the nanocrystalline GaN on quartz shows bandgap of $3.45 \mathrm{eV}$........162 


\section{CHAPTER 1}

\section{INTRODUCTION}

The invention of first transistor using Ge crystal in 1947 at Bell Labs and later the integrated circuits (IC) in 1950s spurred a new industry, i.e., semiconductor industry. The world has dramatically changed since then. Now there are billions of transistors produced each day and more than $95 \%$ of them are made of silicon. Silicon excels in this field due to its three unique properties: easy availability, stable insulating oxide, easy p-type and ntype doping.

There are still several drawbacks with silicon as a semiconductor material. First, $\mathrm{Si}$ is an indirect bandgap material which has very low quantum efficiency of converting electron energy into light. Secondly, silicon has a bandgap of $1.1 \mathrm{eV}$ at room temperature. So, the thermally excited electrons thus limit the performance to temperatures less than $100{ }^{\circ} \mathrm{C}$. Thirdly, the carrier mobility of silicon is low and not suitable for high-speed electronics. What could be the replacement for silicon? They are III-V, II-VI compound semiconductors. The most widely used compound semiconductor is GaAs since the bulk crystals can readily be produced though they are ten times more expensive than silicon. These compound semiconductors have been widely used in light emitting devices such as LEDs and LDs, high temperature electronics, high power as well as high frequency devices. Wireless revolution is an excellent example of these successes. 
Most compound semiconductors including GaAs shown in Region I of Fig. 1.1 have bandgap less than $3 \mathrm{eV}$. The next generation of solid state lighting and blue light emitting devices are critical for producing white LEDs for replacing light bulbs invented more than a century ago. ZnSe, a material with a bandgap of $2.7 \mathrm{eV}$, was the research focus for blue emitting devices in the late $80 \mathrm{~s}$ and early $90 \mathrm{~s}$ of last century. SiC is another indirect bandgap material for blue to UV emitting devices. However, the quantum efficiency of III-nitrides surpasses the performance of $\mathrm{ZnSe}$ and $\mathrm{SiC}$ based devices. For example, the life time of laser diodes (LD) made using high density defect $\left(10 \% \mathrm{~cm}^{2}\right)$ InGaN is almost comparable to low defects $\left(10^{3} / \mathrm{cm}^{2}\right) \mathrm{ZnSe}$ based LDs [Nakamura \& Fasol, 1997]. Also III-nitrides including AlN, GaN, and InN form a continuous alloy system (InGaN, InAlN, AlGaN) whose direct bandgap range from $0.7 \mathrm{eV}(1770 \mathrm{~nm})$ for $\mathrm{InN}$ and $3.4 \mathrm{eV}(365 \mathrm{~nm})$ for $\mathrm{GaN}$, to $6.2 \mathrm{eV}(200 \mathrm{~nm})$ for AlN which covers the spectrum from infrared to far UV region.

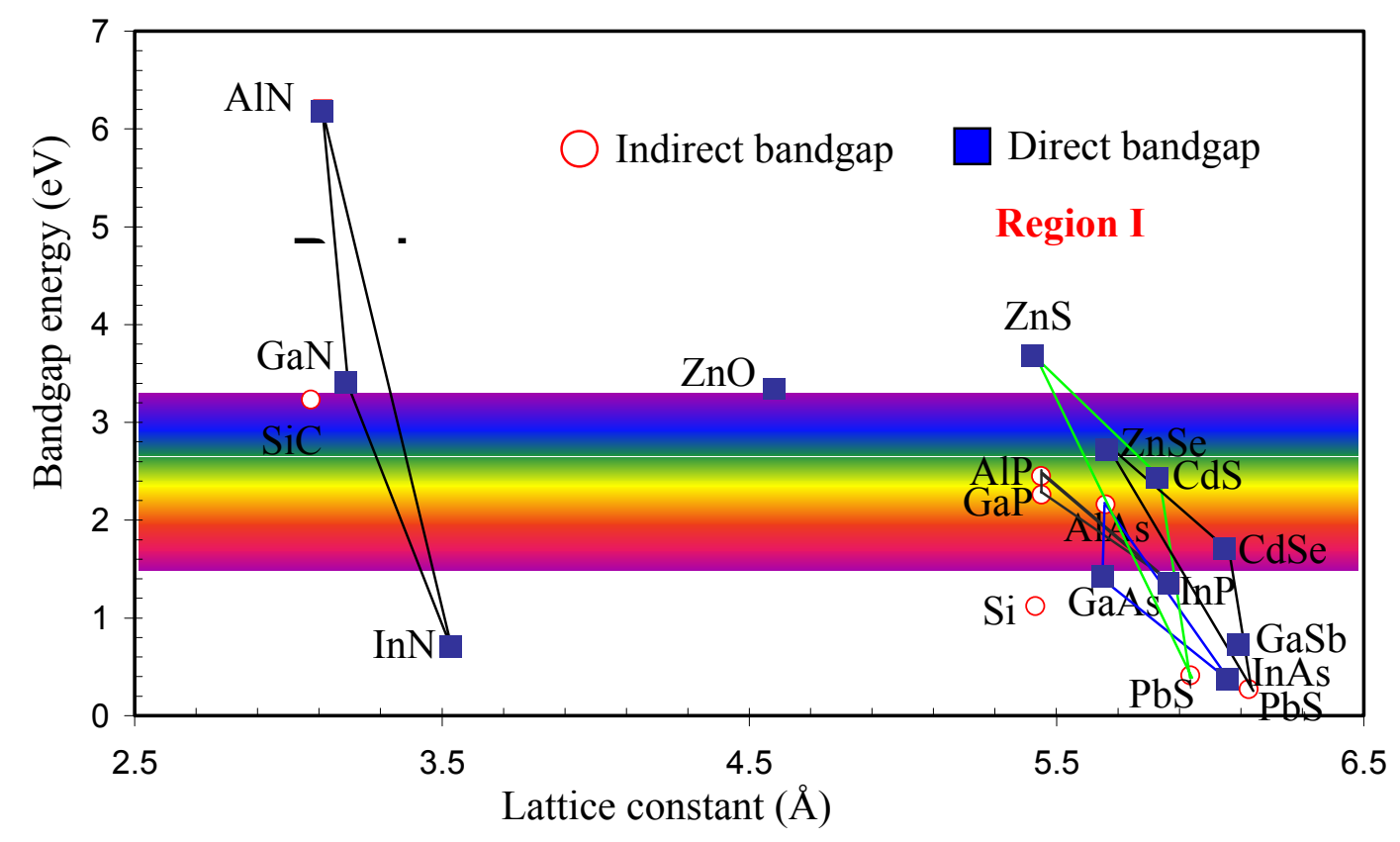

Figure 1.1 The lattice constant and band gap of common semiconductor materials. [Nakamura \& Fasol, 1997] 
Table 1.1 Comparison of properties of several common semiconductor materials.

\begin{tabular}{|c|c|c|c|c|}
\hline & $\mathrm{Si}$ & GaAs & $\mathrm{SiC}$ & $\mathrm{GaN}$ \\
\hline Bandgap (eV) & 1.12 & 1.42 & $3.0-3.2$ & 3.4 \\
\hline Electron mobility $\left(\mathrm{cm}^{2} / \mathrm{V} \cdot \mathrm{s}\right)$ & $\leq 1400$ & $\leq 8500$ & $\leq 1000$ & $\leq 1000$ \\
\hline Thermal conductivity $(\mathrm{W} / \mathrm{cm} \cdot \mathrm{K})$ & 1.3 & 0.55 & $3-5$ & 1.3 \\
\hline Breakdown voltage $\left(\times 10^{6} \mathrm{~V} / \mathrm{cm}\right)$ & 0.6 & 0.65 & $2-3$ & 5 \\
\hline Saturation velocity $\left(\times 10^{7} \mathrm{~cm} / \mathrm{s}\right)$ & 1.0 & 1.0 & 2.0 & 3.0 \\
\hline Melting point $\left({ }^{\circ} \mathrm{C}\right)$ & 1415 & 1238 & 2830 & 2500 \\
\hline \multicolumn{5}{|l|}{ The Johnson Figure of merit } \\
\hline (handling high frequencies & 1 & 10 & 400 & 790 \\
\hline \multicolumn{5}{|l|}{ capacity) } \\
\hline The Baliga figure of merit & & & & \\
\hline (high-power handling capability) & 1 & 17 & 33 & 100 \\
\hline
\end{tabular}

Ever since the first demonstration of blue LED based on III-nitrides in early 1990s, the research on group III-nitrides (AlN, GaN and InN) has been gaining worldwide attention. Furthermore, GaN based electronics are superior over GaAs based devices in several ways:

(1) GaN devices can withstand much higher electric field than that of GaAs devices due to its larger bandgap of $3.4 \mathrm{eV}$. High breakdown voltage is critical for high power and high frequency electronic devices.

(2) GaN possesses high thermal stability. 
(3) III-nitrides are not toxic compared to II-VI. The arsenic from GaAs is very toxic.

Properties of several common semiconductor materials are collected from internet and are listed in Table 1.1. The comparison between $\mathrm{GaN}$ and other semiconductor materials shows that the figure of merit of GaN for high power, high frequency applications is the highest of any semiconductor material excluding that of diamond. The device market based on III-nitrides has dramatically increased since the first GaN based blue LED in 1992 and the first GaN-based blue laser in 1997. Silicon is still going to dominate the semiconductor industry but III-nitrides are going to find their applications where other materials fail to work. In 2004, the III-Nitrides industry was estimated as $\$ 3.2$ billion in revenues, and is expected to grow to $\$ 7.2$ billion in 2009 according to a report from Strategies Unlimited [Strategies Unlimited Report, 2005]. There are estimates that GaN market will reach about $20 \%$ of the whole semiconductor market within next 10 years. III-nitrides materials growth is one of the fundamentals to fully achieve these goals. Epitaxial growth of gallium nitride $(\mathrm{GaN})$ on lattice-mismatched substrates such as sapphire is the state-of-the-art technology for devices fabrications [Liu \& Edgar, 2002]. High density of dislocations in the resulting films due to the lattice mismatch severely degrades the performance (efficiency and lifetime) of devices. Therefore the pursuit of large area, low defect-density single crystal GaN has been the key to achieve the full potential for GaN-based devices.

In addition, the miniaturization of electronic devices has pushed optical lithography technique to its limit and necessitates the creation of bulk nanoscale materials with dimension less than $50 \mathrm{~nm}$. Nanowires, which are one-dimensional nanostructures, 
have become interesting as building blocks for nanoelectronic devices. Also at nanoscale, the materials could exhibit different properties compared to their bulk properties. Examples include the increase in bandgap with decrease in nanoparticle sizes [Wilson, W.L., et al., 1993] and thermal conductivity decrease with shrinking of size [Mingo, 2004]. Quantum confinement, which is the confinement of excitons within the physical boundary of the semiconductor when the physical dimension is less than twice the Bohr radius of the exciton, is also of great interest. These emerging nanostructures and their integration into devices are expected to have an impact. However, controlled growth is critical for integration of nanowires into practical applications, especially for anisotropic crystals like GaN. Therefore, understanding the nucleation and growth kinetics of nanostructures are important to reach these goals.

This thesis presents two concepts: (a) growing large area, near single crystal quality bulk gallium nitride $(\mathrm{GaN})$ crystals and (b) controlled $\mathrm{GaN}$ nanowire synthesis based on bulk nucleation and growth for GaN crystals. In the first concept, we hypothesized that the growth of $\mathrm{GaN}$ crystals from molten gallium would self-align with respect to each other due to the mobility of nitrogenated gallium and form a highly oriented film directly on gallium melt. The self-oriented crystal growth on molten gallium is expected to yield low density of defects $\mathrm{GaN}$.

In the second concept, we hypothesized that the spontaneous nucleation and growth of $\mathrm{GaN}$ crystals from molten gallium could be affected to obtain $\mathrm{GaN}$ nanowires. Further, it is proposed to produce large single crystal using homo-epitaxy onto nanowires as seeds. 
The thesis is organized as the following: the second chapter will detail the fundamental properties of gallium nitride and discuss the state-of-the-art GaN crystal growth techniques. Chapter 3 discusses our proposed concepts of self-oriented GaN crystal growth on molten gallium and controlled GaN nanowires growth. Chapter 4 gives the procedures of the experiments and various materials characterization techniques. Chapter 5 discusses the results of self-oriented $\mathrm{GaN}$ on molten $\mathrm{Ga}$ and chapter 6 will highlight the results on controlled synthesis of GaN nanowires. Chapter 7 describes the homo-epitaxial studies on GaN nanowires grown in different directions. Chapters $8 \& 9$ summarize the results with conclusions and suggest future directions resulting from the research conducted in this work. 


\section{CHAPTER 2}

\section{BACKGROUND}

\subsection{Gallium Nitride (GaN)}

Gallium nitride $(\mathrm{GaN})$ is a group III-V compound semiconductor with a direct band gap of $3.4 \mathrm{eV}$ (which corresponds to a wavelength of $365 \mathrm{~nm}$ ). It is an attractive material for applications in opto-electronics as well as high temperature, high power electronics. GaN can alloy with $\mathrm{AlN}$ and $\mathrm{InN}$ over a continuous range of compositions for bandgap engineering from $0.7 \mathrm{eV}$ to $6.2 \mathrm{eV}$, which is essential for producing any specific wavelength emitters. The unique properties of gallium nitride are closely related to its crystal structure.

\subsubsection{Crystal Structure}

The Ga-N bond in gallium nitride crystal is strongly ionic and the bonding energy is $8.9 \mathrm{eV}$. The strong ionic bond makes gallium nitride very stable and has a melting point of $2500{ }^{\circ} \mathrm{C}$ [Van Vechten, 1973]. Gallium nitride has two different crystal structures. The normally stable structure is hexagonal close-packed (HCP) structure which is one of the two ways where spheres can be packed together in space with the greatest possible density and still have a periodic arrangement. The structure is shown in Fig. 2.1(a). There are two lattice points per unit cell, one is at $(0,0,0)$ and the other one is at $\left(\frac{2}{3}, \frac{1}{3}, \frac{1}{2}\right)$ (or $\left(\frac{1}{3}, \frac{2}{3}, \frac{1}{2}\right)$, which is equivalent). Another representation of the HCP structure is shown 
in Fig. 2.1 (b), the three atoms in the interior of the hexagonal prism are directly above the centers of the alternate triangle in the base. The lattice parameter of $\mathrm{HCP} \mathrm{GaN}$ is $\mathrm{a}_{1}=$ $\mathrm{a}_{2}=3.1887 \AA, \mathrm{c}=5.1853 \AA$. The ratio of $\mathrm{c} / \mathrm{a}$ is 1.626 , which is very close to the standard c/a ratio of 1.633 for hexagonal lattice made of spheres.

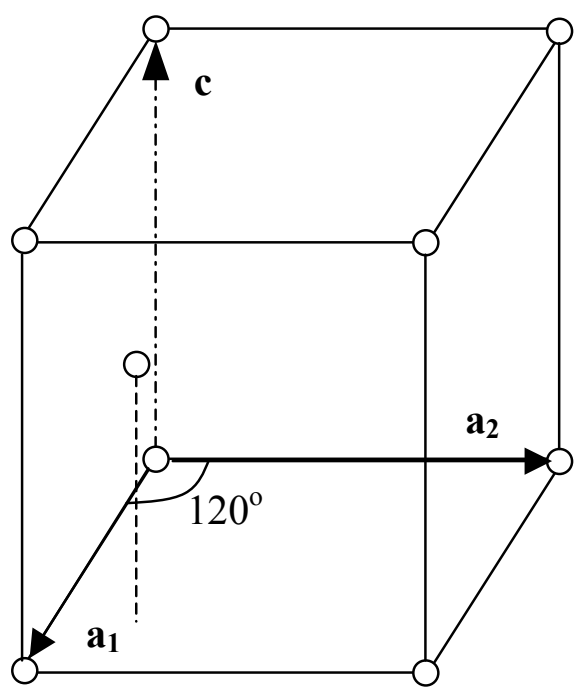

(a)

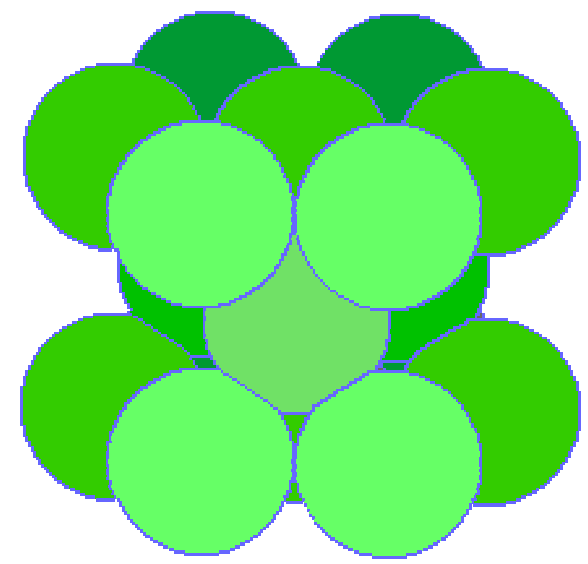

(b)

Figure 2.1 (a) \& (b) Structure of HCP lattice.

As seen in Fig. 2.1(a), the HCP structure of gallium nitride belongs to the primitive hexagonal lattice with

$$
a_{1}=a_{2} \neq c, \quad \alpha=\beta=90^{\circ}, \quad \gamma=120^{\circ}
$$

According to the Pearson symbol [Pearson, 1967] which indicates the crystal symmetry and the number of atoms in the unit cell, the HCP structure for $\mathrm{GaN}$ is designated as hP4. The "h" means "hexagonal", the capital "P" indicates that the centering is "primitive", the number "4" stands for the number of atoms in the unit cell of wurtzite GaN.

The point group representing the symmetry in two-dimensional lattice for $\mathrm{GaN}$ hexagonal lattice is $6 \mathrm{~mm}$. The " 6 " means 6 -fold rotational axial symmetry with the axis of rotation perpendicular to the plane of the two-dimensional lattice. The axis is the c- 
axis in the three-dimensional lattice. The both letters " $\mathrm{m}$ " indicates the presence of the mirror reflection symmetry with two mirror planes (lines in two dimensions) parallel to the rotational c-axis. The symmetry elements of point group $6 \mathrm{~mm}$ for hexagonal $\mathrm{GaN}$ are shown in Fig. 2.2.
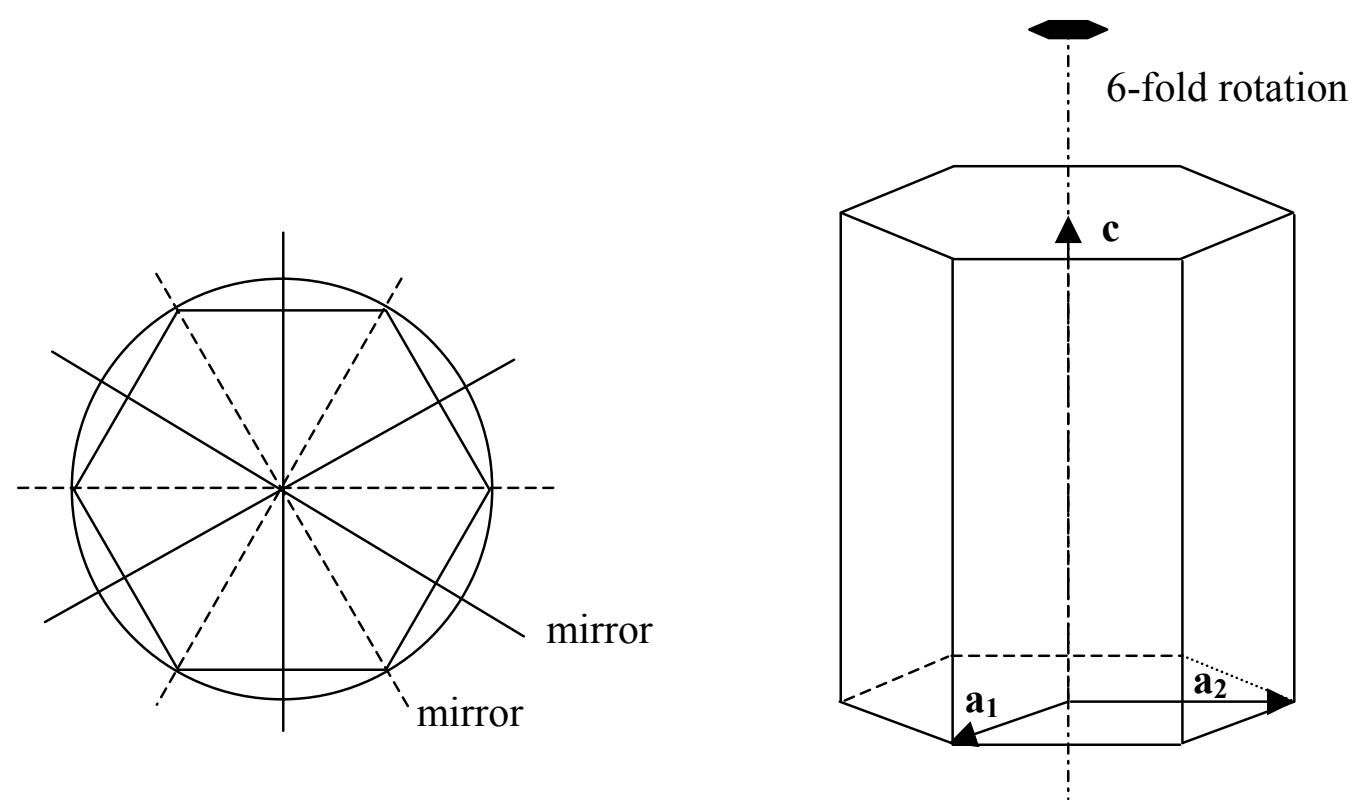

Figure 2.2 Symmetry elements of point group $6 \mathrm{~mm}$ for hexagonal GaN.

A space group involves not only rotation, inversion, and reflection symmetry elements, but also translation [Brown \& Forsyth, 1973]. A combination of rotation and translation can be thought as a symmetry operation with a screw axis, and a combination of reflection and translation can be viewed as an operation with a glide plane of symmetry. The three-dimensional space group of hexagonal gallium nitride lattice belongs to $\mathrm{P} 6_{3} \mathrm{mc}$ (The No. is 186 among total of 230 ). The capital letter "P" is for "primitive". The latter combination of the numbers and letters displays the fact that translation and glide elements are added for a space description of the lattice. The "6 3 " indicates that there is a repetition through rotation on $360 / 6=60^{\circ}$ and translation of $3 / 6 \mathbf{c}$ 
$=\mathbf{c} / 2$. The notation " $m c$ " represents the fact that in three-dimensional sense of the space group, one of the mirror planes has been replaced by the glide plane, which is parallel to the repeat vector $\mathbf{c}$.

For compound $\mathrm{GaN}$ with both $\mathrm{Ga}$ atoms and $\mathrm{N}$ atoms, there are gallium atoms both at $(0,0,0)$ and at $(2 / 3,1 / 3,1 / 2)$. Nitrogen atoms are located at $(0,0, u)$ and $(2 / 3,1 / 3,1 / 2+u)$, where $u$ is 0.377 , which approximately equals to $3 / 8$. The 3 -D view of crystallographic structure of GaN using online software Webemaps [Zuo \& Mabon, UIUC] is shown in Fig. 2.3.

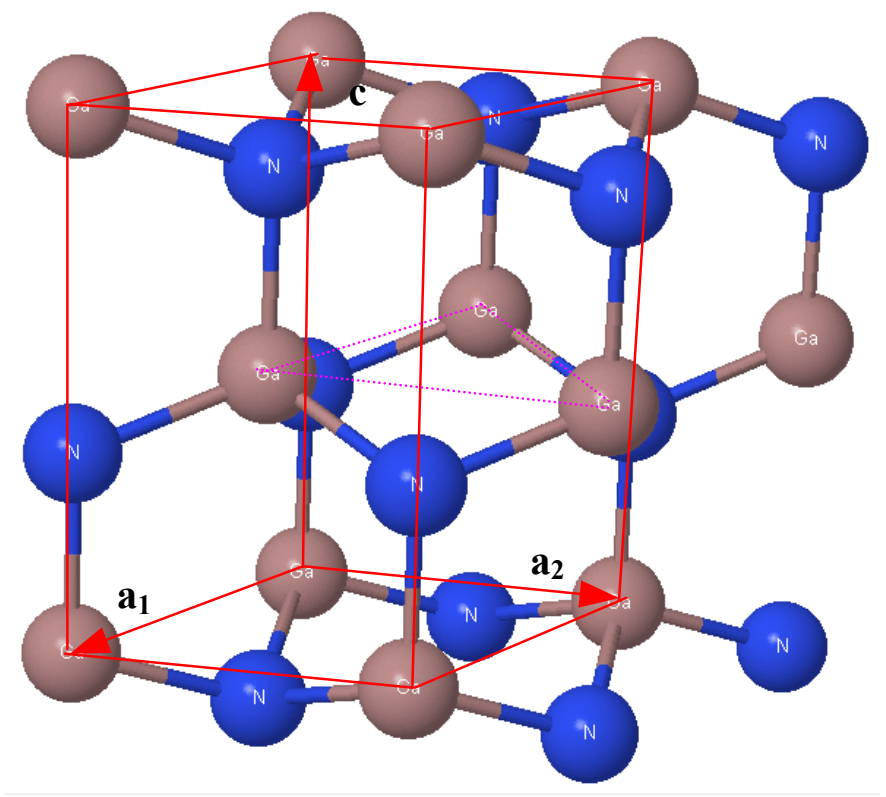

Figure 2.3 3-D view of crystallographic structure of hexagonal GaN.

Another structure of $\mathrm{GaN}$ is the face-centered cubic (FCC) structure which is equally closed packed arrangement with HCP structure. The atoms on the (111) planes of the FCC structure are arranged in a hexagonal pattern just like those atoms on the (0002) planes of the HCP structure. The difference is that the atoms on the third layer are directly above those on the first layer with stacking sequence of ABABA..... for HCP 
structure, while for FCC structure the atoms of the third layer are placed in the hollows of the second layer with stacking sequence of $\mathrm{ABCABC} \ldots .$. The comparison between $\mathrm{HCP}$ and FCC structures are shown in Fig. 2.4.

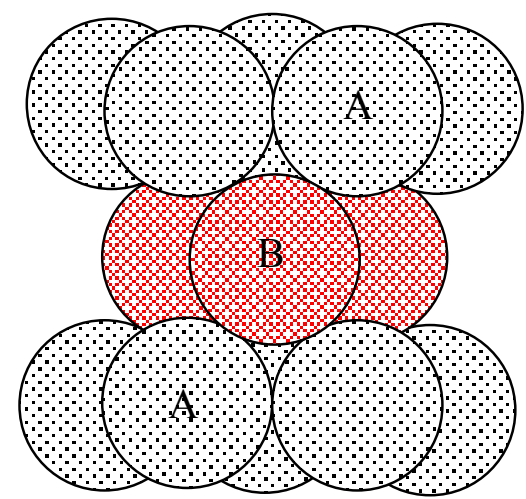

(a)

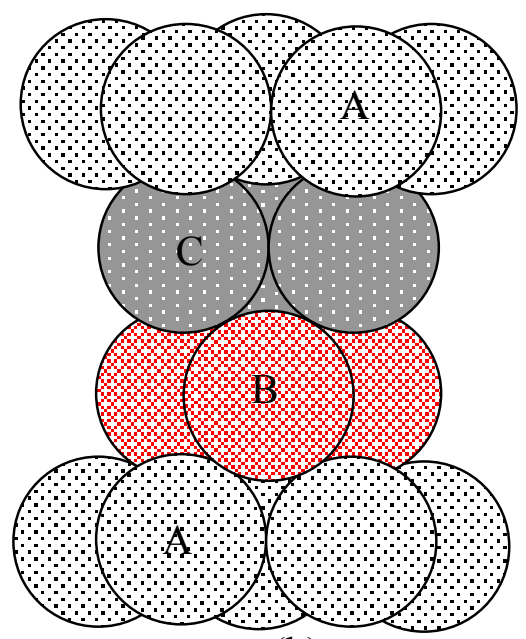

(b)

Figure 2.4 Comparison between HCP and FCC structures: (a) HCP stacking along $<0001>$ direction; (b) FCC stacking along $<111>$ direction.

The Pearson symbol of FCC structure is $\mathrm{cF} 8$. The "c" means "cubic"; the capital "F" stands for "face-centered", the number " 8 " indicates that there are total 8 atoms in the unit cell of cubic $\mathrm{GaN}$, there are $4 \mathrm{Ga}$ and $4 \mathrm{~N}$ atoms respectively.
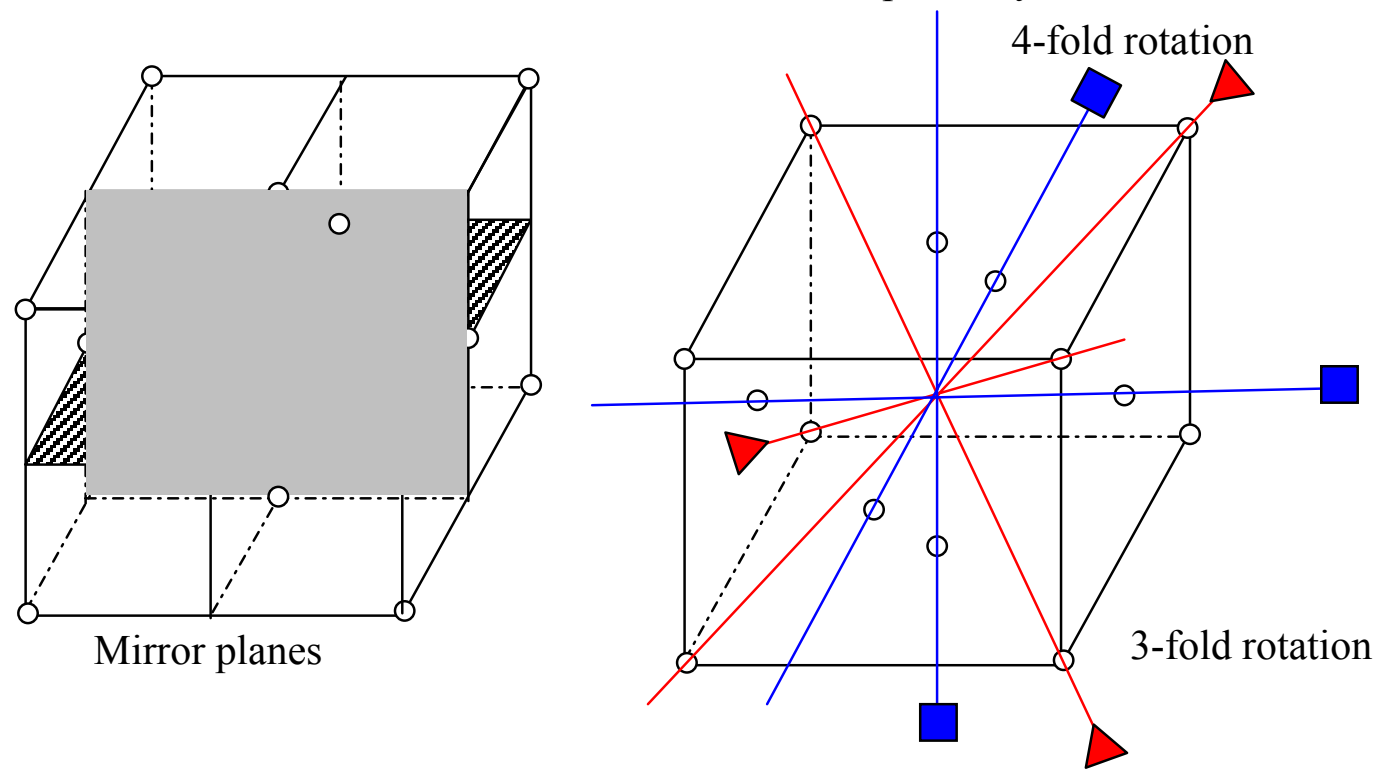

Figure 2.5 The symmetry elements of the FCC structure of cubic GaN. 
The point group of FCC structure is $\overline{4} 3 m$, the " $\overline{4}$ " represents there are 4 fold rotation with simultaneous inversion; the number " 3 " means that there are three fold rotation axis; the " $m$ " indicates the presence of mirror reflection symmetry. The symmetry elements of the FCC structure are shown in Fig. 2.5. The space group of the FCC structure is the No. 216, $F \overline{4} 3 m$.

The unit cell of cubic GaN has 8 atoms, $4 \mathrm{Ga}$ atoms are located at $(0,0,0),(1 / 2$, $1 / 2,0),(1 / 2,0.1 / 2)$, and $(0,1 / 2,1 / 2)$. The positions of $4 \mathrm{~N}$ atoms are $(1 / 4,1 / 4,1 / 4),(3 / 4$, $3 / 4,1 / 4),(3 / 4,1 / 4,3 / 4)$ and $(1 / 4,3 / 4,3 / 4)$. The atom position of cubic $\mathrm{GaN}$ is identical with those in cubic diamond, only difference is one set of positions is $\mathrm{Ga}$, the other set occupied by $\mathrm{N}$ atom is translated along the $<111>$ direction with $\frac{\sqrt{3}}{4}$. The 3-D view of crystallographic structure of cubic GaN is shown in Fig. 2.6.

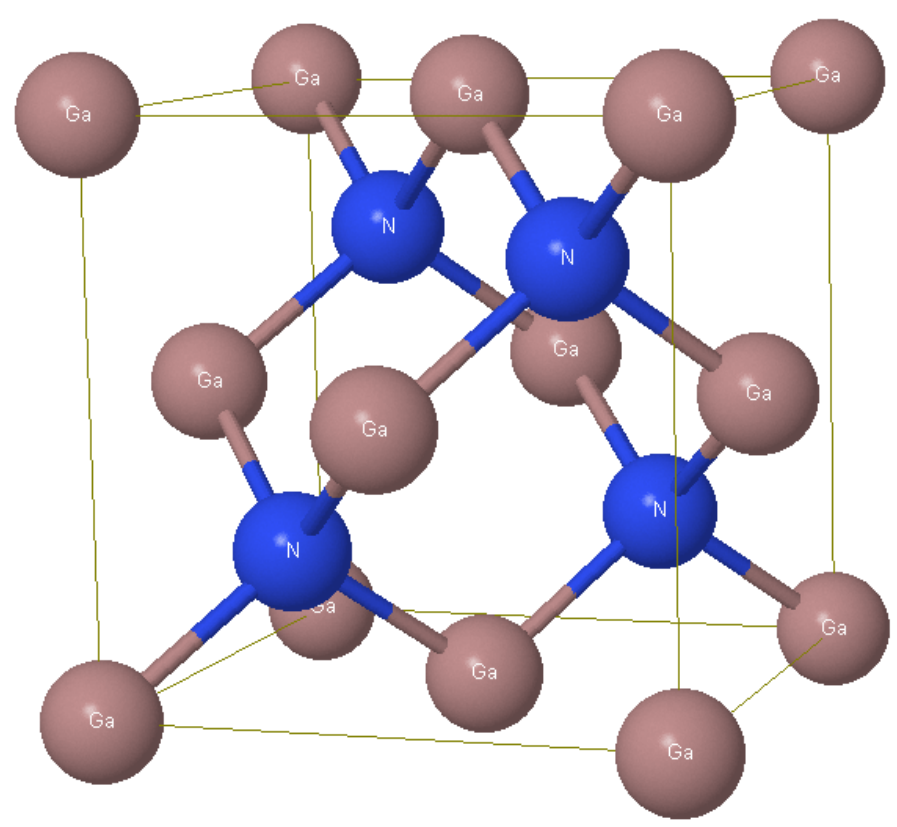

Figure 2.6 3-D view of crystallographic structure of cubic $\mathrm{GaN}$. 


\subsubsection{Fundamental Properties of GaN}

The unique properties of III-nitrides make them so attractive for applications in optoelectronic and electronic devices. The theoretical melting point of $\mathrm{GaN}$ is predicted to be $2500{ }^{\circ} \mathrm{C}$ due to the large ionic bond strength of Ga-N [Van Vechten, 1973]. The wide band gap and good thermal conductivity also contribute to its application in high temperature and high power electronics where devices based on Si or GaAs are not applicable. For example, the GaN based field effect transistor could yield more than 50 times of power capability than that based on GaAs due to the higher breakdown field and larger band gap. The physical and electronic properties of $\mathrm{GaN}$ are listed in Table 1 and 2 respectively.

Table 2.1 Physical properties of GaN.

\begin{tabular}{lccc}
\hline \multicolumn{1}{c}{ Physical Properties (300K) } & $\begin{array}{c}\text { Hexagonal } \\
\text { GaN }\end{array}$ & Cubic GaN & Reference \\
\hline Energy band gap $(\mathrm{eV})$ & 3.39 & 3.2 & Levinshtein, et al. 2001 \\
Density $\left(\mathrm{g} / \mathrm{cm}^{3}\right)$ & 6.15 & 6.15 & Levinshtein, et al. 2001 \\
Melting point $\left({ }^{\circ} \mathrm{C}\right)$ & 2500 & 2500 & Porowski, 1997 \\
Lattice constant $(\AA)$ & $\mathrm{a}=3.189$, & $\mathrm{a}=4.52$ & Levinshtein, et al. 2001 \\
Thermal conductivity & $\mathrm{c}=5.186$ & & \\
$(\mathrm{~W} / \mathrm{cm} \cdot \mathrm{K})$ & 2.1 & 2.1 & Florescu, et al. 2000 \\
Thermal expansion coefficient & $\alpha_{\mathrm{a}}=5.59 \times 10^{-6}$ & & \\
$(1 / \mathrm{K})$ & $\alpha_{\mathrm{c}}=3.17 \times 10^{-6}$ & $\mathrm{~N}$ & Levinshtein, et al. 2001 \\
Thermal diffusivity $(\mathrm{cm} / \mathrm{s})$ & 0.43 & 0.43 & Levinshtein, et al. 2001 \\
Heat capacity $(\mathrm{J} / \mathrm{mol} \cdot \mathrm{K})$ & 35.3 & 35.3 & Krukowski, et al. 1999 \\
Dielectric constant $(\mathrm{static})$ & 8.9 & 9.7 & Levinshtein, et al. 2001 \\
Refractive index & 2.3 & 2.3 & Levinshtein, et al. 2001 \\
Bulk modulus $(\mathrm{GPa})$ & 210 & 210 & Kucheyev, et al. 2000 \\
\hline
\end{tabular}


Table 2.2 Electronic properties of GaN. [Levinshtein, et al. 2001]

\begin{tabular}{lcc}
\hline \multicolumn{1}{c}{ Electronic Properties } & $\begin{array}{c}\text { Hexagonal } \\
\text { GaN }\end{array}$ & Cubic GaN \\
\hline Electron mobility $\left(\mathrm{cm}^{2} / \mathrm{V} \cdot \mathrm{s}\right)$ & $\leq 1000$ & $\leq 1000$ \\
Hole mobility $\left(\mathrm{cm}^{2} / \mathrm{V} \cdot \mathrm{s}\right)$ & $\leq 200$ & $\leq 350$ \\
Effective electron mass $\left(\mathrm{m}_{0}\right)$ & 0.20 & 0.13 \\
Effective hole mass $\left(\mathrm{m}_{0}\right)$ & & \\
$\quad$ Heavy & 1.4 & 1.3 \\
$\quad$ Light & 4.3 & 0.2 \\
Electron affinity $(\mathrm{eV})$ & $\sim 5 \times 10^{6}$ & $\sim 5 \times 10^{6}$ \\
Breakdown field $(\mathrm{V} / \mathrm{cm})$ & & \\
Diffusion coefficient $(\mathrm{cm} / \mathrm{s})$ & 25 & 25 \\
$\quad$ Electrons & 5 & 3.1 \\
$\quad$ Holes & $2.6 \times 10^{5}$ & $9.5 \times 10^{4}$ \\
Electron thermal velocity $(\mathrm{m} / \mathrm{s})$ & $9.4 \times 10^{4}$ & \\
Hole thermal velocity $(\mathrm{m} / \mathrm{s})$ & &
\end{tabular}

\subsubsection{Applications}

The most significant and widely commercialized application of III-nitrides is to fabricate Light Emitting Diodes (LED). LEDs have the advantages of high efficiency and longer life time, which will replace the incandescent lamps as the next generation of lighting. The research on LEDs has been carried out for more than 40 years. As seen from the Fig. 1.1, the III-nitride family itself covers the range of spectrum from near-infrared to visible and far UV. However, the research on nitride based LEDs developed very slowly due to unavailability of bulk substrates, bad quality of the epitaxially grown films and the in-effective p-doping. Until mid-80s of last century, the use of low temperature 
AlN or GaN buffer layer produced smooth, crack free epitaxial GaN film on sapphire substrates [Amano, et al.,1986]. Following that, the successful p-doing GaN was discovered by low-energy electron-beam irradiation (LEEBI) of magnesium-doped GaN [Amano, et al., 1989] and real p-n diode was demonstrated. Another method of producing effective p-doping was found by thermal annealing $\mathrm{Mg}$ doped $\mathrm{GaN}$ under $\mathrm{N}_{2}$ atmosphere [Nakamura, et al.,1992]. The commercial nitride based LEDs quickly came out after these breakthroughs and the white LEDs became possible since all three primary colors LEDs (blue, red and green) are available. The efficiency of LEDs has been increasing ever since and high power LEDs capable of emitting more than $150 \mathrm{~lm} /$ package has recently been fabricated [Steranka, 2002]. The efficiency and output power of LEDs need to be improved further if one were to replace current lighting with solid-state lighting. This can only be done through improvements in the materials quality and other devices fabrication steps.

Ultraviolet (UV) LEDs also have other applications across industry fields such as spectro-fluorometry, photo-catalytic reactions, high-resolution optics, counterfeit detection, chemical detection, medical applications and UV air purifier, etc. The market of LEDs had reached $\$ 2.5$ billion dollars globally in 2002, while growing $20 \%$ each year. It is predicted to reach between 4 and 5 billion dollars by 2007.

According to a study by DOE, the energy savings to date from using of LEDs has exceeded more than 8 billion $\mathrm{kW}$-hours which equals to the power produced from one large electric power plant. It predicts that till 2025, the energy saving associated with solid-state lighting will reach approximately 114 billion $\mathrm{kW}$-hours, which is equivalent to electrical output of about 14 large power plants. 
The III-nitride based laser diodes with shorter wavelength find several applications in data storage and printing. The shorter the wavelength is, the larger storage capacity of a CD has and the higher the resolution of printing. Currently, near infrared laser diode based on AlGaAs with a wavelength of $780 \mathrm{~nm}$ is used to read the $\mathrm{CD}$. The InGaN laser diode with a wavelength of $410 \mathrm{~nm}$ will quadruple the storage capacity of a current CD or DVD. Other applications of laser diode include medical diagnostics and therapy, detection of biological/chemical weapons, projectors, and molding. The worldwide market for laser diodes in non-communications applications is forecast to exceed $\$ 2.2$ billion by 2004 . The biggest obstacle of the III-nitride based laser diode is still the materials, i.e., the lack of high quality materials.

III-nitrides are also good candidates for UV detectors, high power RF electronics and high temperature electronics. III-nitrides based materials perform better than $\mathrm{Si}$ and GaAs and provide a unique set of materials for several applications. Several of the applications mentioned above are still under development and the market is limited. With the progress in the optoelectronics area especially from the materials point of view, the potential market for III-nitride is forecast to reach as high as 5 billion in 2009. Apart from the opto-electronic applications, $\mathrm{GaN}$ is also found to be stable material for $\mathrm{UV}$ induced photon conversion of solar energy for water-splitting reactions [Kocha, S. S., Turner, J. A., et al., 1995].

\subsection{State-of-the-art GaN Crystal Growth}

\subsubsection{Epitaxial Growth}

The epitaxial growth on foreign substrates such as sapphire, silicon, silicon carbide, GaAs, etc has been primarily used for producing $\mathrm{GaN}$ wafers due to non- 
availability of bulk gallium nitride substrates. The different substrates used for GaN epitaxy has been recently reviewed [Liu, L. \& Edgar, J. H., 2002]. The vapor phase epitaxy techniques for $\mathrm{GaN}$ growth include metal organic chemical vapor deposition (MOCVD), molecular beam epitaxy (MBE) and hydride vapor phase epitaxy (HVPE).

\subsubsection{Metal Organic Chemical Vapor Deposition (MOCVD)}

MOCVD, also called MOVPE, is a process which utilizes metalorganic compounds to react with gas source materials to form a solid on substrates. MOCVD has produced the best quality $\mathrm{GaN}$ on sapphire or $\mathrm{SiC}$ to date and is the main stream of commercial process for device fabrication. GaN growth is typically done using reaction between trimethyl gallium (TMGa) and ammonia $\left(\mathrm{NH}_{3}\right)$ :

$$
\left(\mathrm{CH}_{3}\right)_{3} \mathrm{Ga}(\mathrm{g})+\mathrm{NH}_{3}(\mathrm{~g}) \rightarrow \mathrm{GaN}(\mathrm{s})+3 \mathrm{CH}_{4}(\mathrm{~g})
$$

Other source materials include triethylgallium $\left(\left(\mathrm{C}_{2} \mathrm{H}_{5}\right) \mathrm{Ga}\right)$ for gallium precursor and dimethylhydrazine $\left(\mathrm{CH}_{3}\right)_{2} \mathrm{~N}_{2} \mathrm{H}_{2}$ or $\mathrm{HN}_{3}$ for nitrogen source. The actual growth process is rather complex which involves proper heat, mass, and momentum transfer, appropriate gas phase and surface reaction kinetics. Modeling of the growth process has been developed from micro-scale [Hirako, et al., 2002] to reactor scale [Pawlowski, et al., 2000].

The reactor chamber for MOCVD GaN growth has to be designed specially due to the complicated reactions. The formation of adduct between $\mathrm{TMGa}$ and $\mathrm{NH}_{3}$ has to be prevented prior to their interaction with the substrate. The high pressure of $\mathrm{NH}_{3}$ is necessary to overcome the decomposition of GaN film and this requirement induces thermal convection at the high reaction temperature used, which makes the film thickness nonuniform over the substrates. Different designs have been developed to overcome these 
problems such as two-flow reactors [Nakamura, et al., 1991], three-flow reactor [Wang, et al., 2000], as well as rotating substrate stage reactor [Kadinski, et al., 2004].

The growth parameters for MOCVD GaN on sapphire have been optimized over a number of years. The substrate is annealed at high temperature around $1100{ }^{\circ} \mathrm{C}$ followed by a nucleation layer such as AIN [Widmann, et al., 1999] or GaN [Nakamura, 1991] deposited at $500-600{ }^{\circ} \mathrm{C}$. The nucleation layer is critical to achieve layer-by-layer growth and flat surface. Then the nucleation layer is annealed at high temperature and final growth is conducted under a pressure of $40-300$ torr, temperature from $1050^{\circ} \mathrm{C}$ to 1250 ${ }^{\circ} \mathrm{C}$. The growth rate under these conditions is from $1-5 \mu \mathrm{m} / \mathrm{h}$. The $\mathrm{n}$-type doping gas source is typically $\mathrm{SiH}_{4}$ or $\mathrm{Si}_{2} \mathrm{H}_{6}$ and n-type doping gas source is Bis (cyclopentadienyl) magnesium $\left(\mathrm{Cp}_{2} \mathrm{Mg}\right)$. n-type $\mathrm{GaN}$ is easy to achieve but the $\mathrm{Mg}$ doped $\mathrm{GaN}$ needs special treatment due to the passivation of $\mathrm{Mg}$ acceptor by hydrogen [Ohba, \& Hatano, 1994]. Though both low energy electron beam irradiation (LEEBI) [Amano, et al., 1989] and annealing in $\mathrm{N}_{2}$ [Nakamura, et al., 1992] have succeeded in activating the Mg acceptor, $\mathrm{p}$ type GaN with high mobility and high doping concentration is still a challenging task [Doverspike \& Pankove, 1998].

\subsubsection{Molecular Beam Epitaxy (MBE)}

Molecular beam epitaxy (MBE) is a growth technique of depositing semiconductor materials under ultrahigh vacuum (UHV) conditions. The absence of gas phase reactions under UHV conditions allows for precise control of the composition and thickness of the resulting films. It has been widely used in commercial production of IIIarsenide, III-phosphide based electronic devices such as MESFETs, HEMTs, MMICs. The MBE growth of III-nitrides, activated nitrogen species produced by plasma sources 
such as electron cyclotron resonance (ECR) microwave source [Oberman, 1995], radio frequency $(\mathrm{RF})$ source has to be used due to the inert nature of nitrogen. The excited nitrogen species and gallium from the effusion cell come to the heated substrate and react to form $\mathrm{GaN}$ :

$\mathrm{Ga}(\mathrm{l})+\mathrm{N}^{*}(\mathrm{~g}) \rightarrow \mathrm{GaN}(\mathrm{s}) ; \mathrm{N}^{*}$ denotes activated nitrogen species The typical chamber for MBE GaN is shown in Fig. 2.7. Other source materials include $\mathrm{NH}_{3}$ for nitrogen and metalorganic precursor for gallium which results in some hybrid techniques, i.e., chemical beam epitaxy (CBE) [Tsang, 1984], metal organic MBE (MOMBE) [Abernathy, 1995].

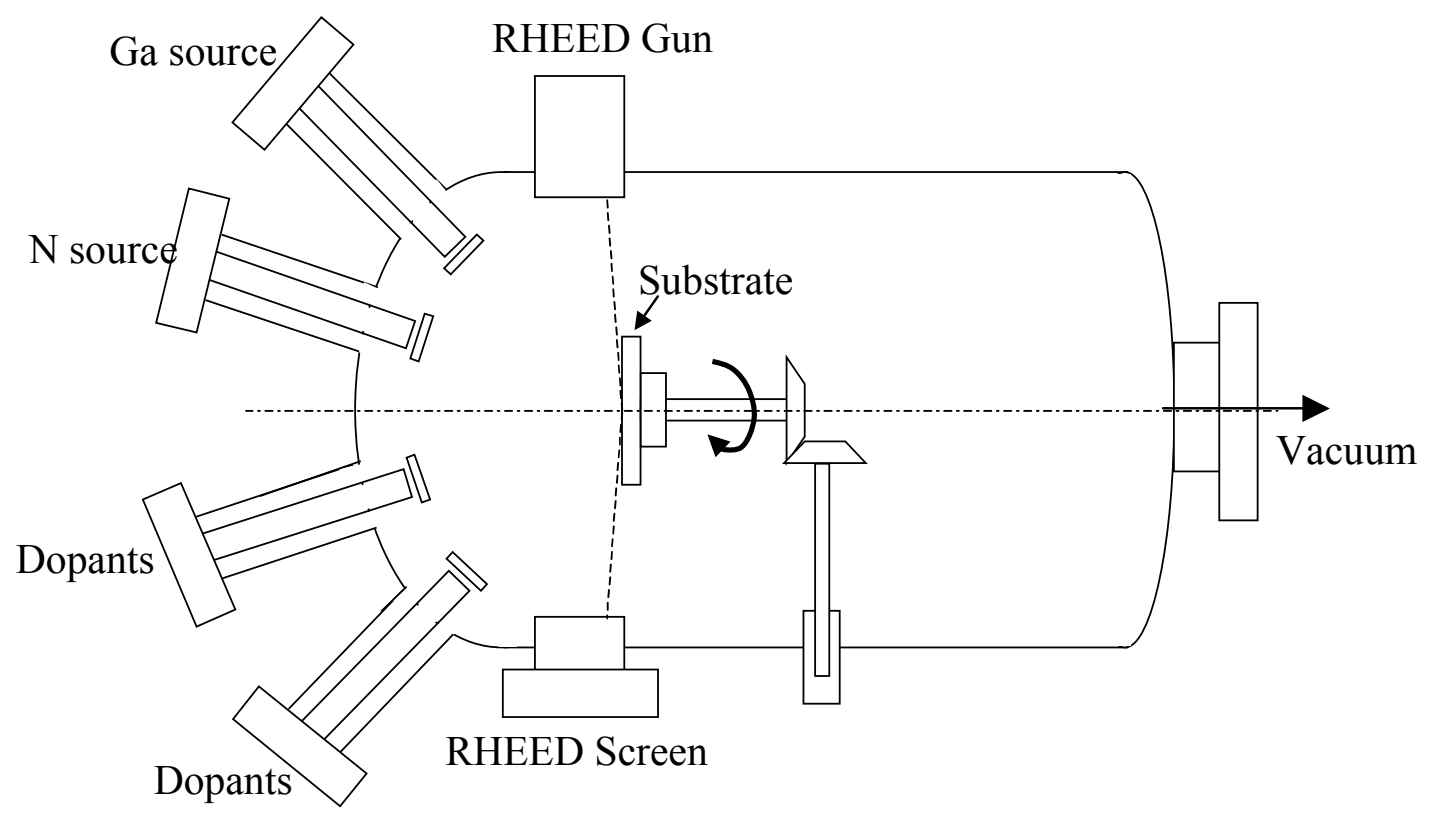

Figure 2.7 Typical chambers for MBE GaN growth.

The MBE GaN growth usually occurs at a temperature of $600-800{ }^{\circ} \mathrm{C}$ and a pressure around $10^{-4}$ torr. The typical growth rate is $0.3-1 \mu \mathrm{m} / \mathrm{h}$, meaning an atomic layer at a time. The growth can be monitored in-situ by Reflection High Energy Electron Diffraction (RHEED) [Held, et al., 1998] or Low Energy Electron Diffraction (LEED) [Pavlovska, 2001]. 
The substrates for MBE GaN growth need to be cleaned and degassed to obtain atomically clean surface to get high quality film [Ruterana, et al., 1997]. The ratio between Ga and nitrogen has significant effects on the surface morphology, structure and optical properties [Haus, et al., 2002]. It is well known that Ga-rich condition is necessary to get smooth (0001) terminated GaN surface in MBE growth and N-rich condition results in rough surface and faceted growth [Heying, et al., 2000; Ramachandran, et al., 2000]. The Ga adlayer reduces the surface energy of (0001) plane and the growth changes to quasi liquid phase eppitaxy (LPE) [Pavlovska, 2001].

The doping of GaN in MBE growth is conducted by using effusion cells containing the dopant source. $\mathrm{Si}$ is the common n-type donor and $\mathrm{Mg}$ acts as the main $\mathrm{p}$ type acceptor [Davis, et al., 1997]. Post-growth process is not needed for MBE grown ptype GaN due to the absence of hydrogen and low temperature growth conditions used. High density incorporation of Mg could deteriorate the film quality [Pezzagna, 2004] and better understanding of this process is needed.

\subsubsection{Hydride Vapor Phase Epitaxy (HVPE)}

Hydride vapor phase epitaxy (HVPE) is a growth technique widely used in semiconductor materials growth. It was the first method to produce epitaxial GaN layer [Maruska, H. P., 1969]. The ability of growing thick GaN with high growth rate makes HVPE as an unique technique compared with above mentioned slow growth rate techniques for producing qausi-bulk GaN substrates [Kim, et al., 1998]. Two-inch GaN wafers with thickness of $500 \mu \mathrm{m}$ have been achieved [Motoki, et al., 2002].

The typical reactor for HVPE shown in Fig. 2.8 usually has several different zones whose temperature can be controlled separately. For GaN growth, the source 
materials $\mathrm{HCl}$ and $\mathrm{NH}_{3}$ are injected into the reactor through the source zone with $\mathrm{N}_{2}$ as a carrier gas. $\mathrm{HCl}$ passes through a crucible contains metallic $\mathrm{Ga}$ in the source zone to form $\mathrm{GaCl}$. Both $\mathrm{GaCl}$ and $\mathrm{NH}_{3}$ are transported to the growth zone and react on the substrate to form $\mathrm{GaN}$. The chemical reactions are:

$$
\begin{aligned}
& 2 \mathrm{Ga}(\mathrm{l})+2 \mathrm{HCl}(\mathrm{g})----2 \mathrm{GaCl}(\mathrm{g})+\mathrm{H}_{2}(\mathrm{~g}) \\
& \mathrm{GaCl}(\mathrm{g})+\mathrm{NH}_{3}(\mathrm{~g})----\mathrm{GaN}(\mathrm{s})+\mathrm{HCl}(\mathrm{g})+\mathrm{H}_{2}(\mathrm{~g})
\end{aligned}
$$

The substrates are usually kept at $950{ }^{\circ} \mathrm{C}$ to $1050{ }^{\circ} \mathrm{C}$ and the pressure of the chamber is about atmosphere pressure.

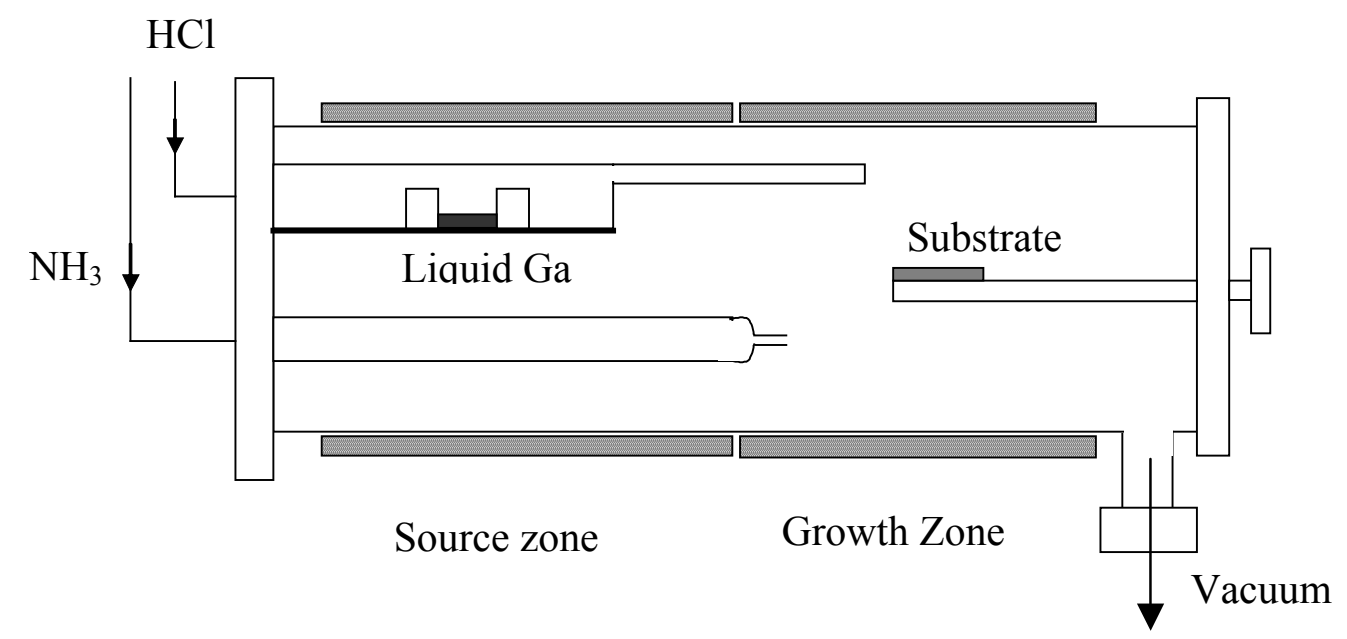

Figure 2.8 Typical reactors for HVPE GaN growth.

Unlike MOCVD and MBE growth, HVPE growth of GaN is an equilibrium process [Cadoret, R. \& Trassoudaine, A., 2000]. The vapor phase composition, the temperature of different zones determines the growth rate and solid composition of the epitaxial layer. The surface kinetics such as absorption, desorption and surface diffusion also play a role in the growth. The mechanism of HVPE growth has been systematically studied [Cadoret, 1999]. 
There are several drawbacks associated with HVPE growth. The corrosive nature of $\mathrm{HCl}$ requires special consideration of materials for the reactor chamber. Different adducts formed inside the chamber are dangerous and could clog the exhaust line. The hot wall nature of the reactor also limits the $\mathrm{p}$ type doping using metalorganic precursors. The advantages and disadvantages of all three vapor phase epitaxy growth techniques are summarized in Table 2.3.

Table 2.3 Comparisons of the three GaN vapor phase epitaxy growth techniques.

\begin{tabular}{|c|c|c|}
\hline & Advantages & Disadvantages \\
\hline \multirow{6}{*}{$\mathrm{MBE}$} & Abrupt interface & Need ultra-high vacuum \\
\hline & Good control of layer composition & Low throughput \\
\hline & Growth at a little lower temperatures & Temperature need to be closely controlled \\
\hline & In-situ RHEED characterization & Very low growth rate \\
\hline & Low contamination & Very expensive process \\
\hline & Free of hydrogen & \\
\hline \multirow{6}{*}{ MOCVD } & High growth rate & Highly toxic gases \\
\hline & High crystal quality & Consume large amount of $\mathrm{NH}_{3}$ \\
\hline & High throughput & Lack of in-situ characterization process \\
\hline & Near atmospheric pressure & Carbon contamination \\
\hline & Sharp interface & Complex and difficult for $p$-doping \\
\hline & Very high growth rate & Handling corrosive gas \\
\hline \multirow[t]{2}{*}{ HVPE } & Low manufacturing cost & Difficult in thickness control \\
\hline & Carbon free & \\
\hline
\end{tabular}




\subsubsection{Defects Engineering Techniques}

The unavailability of bulk GaN substrates necessitates that most of the GaN for device fabrication is grown on sapphire (lattice mismatch $\sim 14 \%$ ) or $\mathrm{SiC}$ (lattice mismatch $\sim 3 \%$ ) substrates. This lattice mismatch between $\mathrm{GaN}$ and the substrates causes high density of misfit dislocations in excess of $10^{10} / \mathrm{cm}^{2}$. So, there has been a search for a suitable lattice matching substrate to reduce defects in the epitaxial grown $\mathrm{GaN}$ layers. Also, there have been attempts to engineer growth processes for reducing defects densities in epitaxial grown $\mathrm{GaN}$. One of the techniques is lateral epitaxial growth (LEO) which has reduced the dislocation density down to $10^{7} / \mathrm{cm}^{2}$. The reviews about LEO of GaN have been published [Gibart, P., 2004].

In $\mathrm{LEO}$ technique, a layer of $\mathrm{GaN}$ is first deposited and followed by partially masking the $\mathrm{GaN}$ with a dielectric material such as $\mathrm{SiO}_{2}$ or $\mathrm{Si}_{3} \mathrm{~N}_{4}$. Then the regrowth starts only within the openings but not on the masks. The threading dislocations are prevented from penetrating into the overgrown layer by the dielectric mask though they are still present in the GaN grown directly above the openings. In order to get low dislocation density GaN allover the whole substrate, a second step LEO can be applied by masking the openings in the first step. The sketch of the LEO processes is shown in Fig. 2.9. 


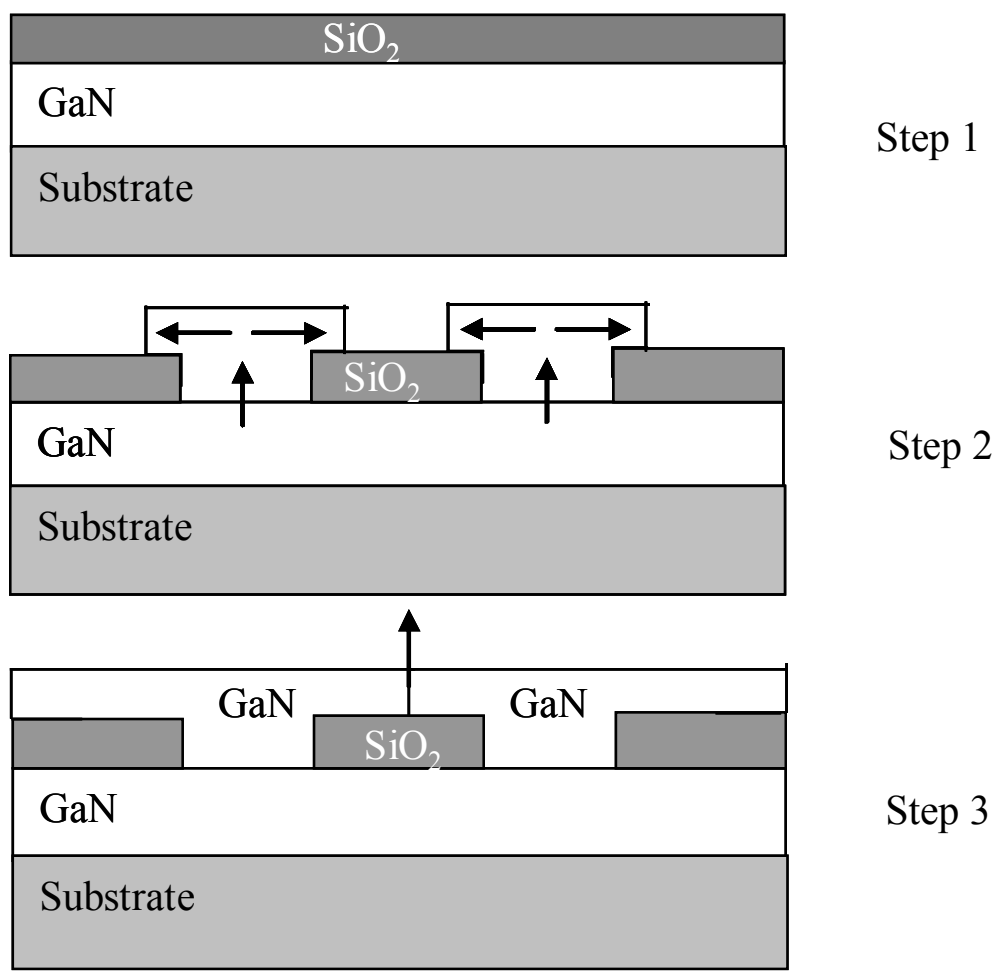

Figure 2.9 Sketch of the LEO process for GaN growth.

The success of LEO relies on the growth only in the openings but not on the masks. This could be achieved when the supersaturation of growth species on the mask is sufficiently low. The growth parameters such as temperature, pressure, and concentration of growth species have significant effect on suppression growth on the masks. The growth facets in the LEO process are also very important to get a uniform, smooth surface and the complete coalescence. The equilibrium facets of $\mathrm{GaN}$ depend on the crystallographic orientation and shapes of the openings [Nam, et al., 1997], temperature [Kitamura, et al., 1995], pressure [Akasaka, et al., 1998], carrier gas [Miyake, et al., 2002], dopants [Beaumont, et al., 1998] as well as V/III ratio [Nam, et al., 1997]. It has 
been found that the lateral growth is easier for stripe opening along $<1-100>$ rather than $<11-20>$ direction [Kapolnek, et al., 1997].

Improvements and revision of LEO technology has also been developed to control the strain and impurities in the overgrown GaN. Maskless LEO [Smart, et al., 1999], pendeo-epitaxy [Zheleva, et al., 1999] and air-bridged LEO [Kidoguchi, et al., 2000] have been reported.

\subsection{Road Map of GaN Crystal Growth}

Hetero-epitaxy directly on single crystal substrates with large lattice mismatch inherently leads to high density of misfit dislocations on the order of $10^{10}-10^{11} \mathrm{~cm}^{-2}$ [Ning, et al, 1996]. Also, the differences in the thermal expansion coefficient between $\mathrm{GaN}$ and the substrates induce thermal stresses in the GaN layer [Nikitina, et al., 1997]. The stresses and high density defects reduce the efficiency and performances of devices [Cao, Pearton, \& Ren, 2000]. Thick GaN films with very high growth rates by hydride vapor phase epitaxy (HVPE) [Morkoc, 2001] and lateral epitaxial overgrowth (LEO) [Gibart, 2004] have reduced the dislocation density down to the order of $10^{7} \mathrm{~cm}^{-2}$. High

power devices such as lasers and transistors require dislocation density less than $10^{4} \mathrm{~cm}^{-2}$ and the only solution is homo-epitaxy on bulk GaN single crystals [Leszczynski, et al., 1999]. The road map for development of GaN wafers is shown in Fig. 2.10 as a function of dislocations density. 


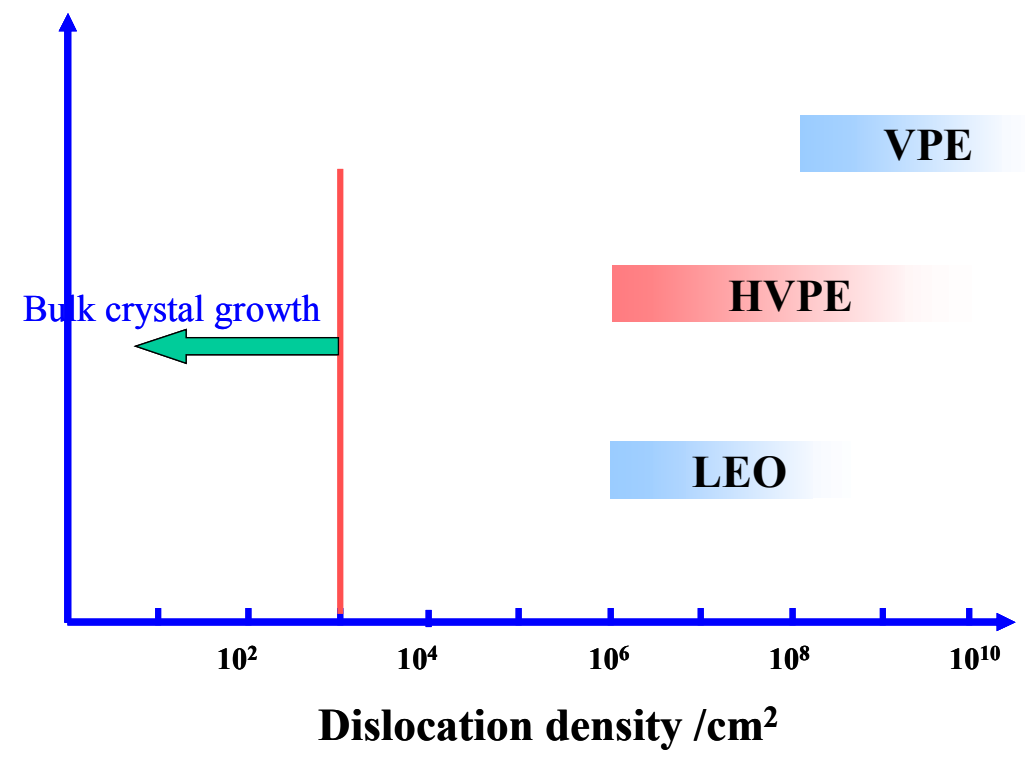

Figure 2.10 Road map showing the need for bulk growth techniques for defects reduction.

\subsubsection{Bulk Single Crystal Growth}

Research on bulk single crystal growth of gallium nitride has been carried out for over 40 years. Due to the high stability of nitrogen molecules and its low solubility in gallium, conventional crystal growth techniques such as Czochralski or Bridgman methods could not be applied for GaN growth. Several bulk GaN crystal synthesis technique have been conducted in laboratories and the size of the crystals is only on $\mathrm{mm}$ scale.

\subsubsection{High Pressure Solution Growth}

The strong bonding of GaN results in a very high melting point which is predicted theoretically to be $2500{ }^{\circ} \mathrm{C}$. [Van Vechten, 1973] The thermodynamic properties of Ga$\mathrm{N}_{2}$-GaN system had been studied by J. Karpinski [Karpinski, et al., 1984] and the phase diagram is shown in Fig. 2.11. The reaction between liquid gallium and molecular 
nitrogen requires a high temperature $\left(>1500{ }^{\circ} \mathrm{C}\right)$ and a high pressure $(>15 \mathrm{kbar})$ due to the inert nitrogen and low solubility of $\mathrm{GaN}$ in gallium.
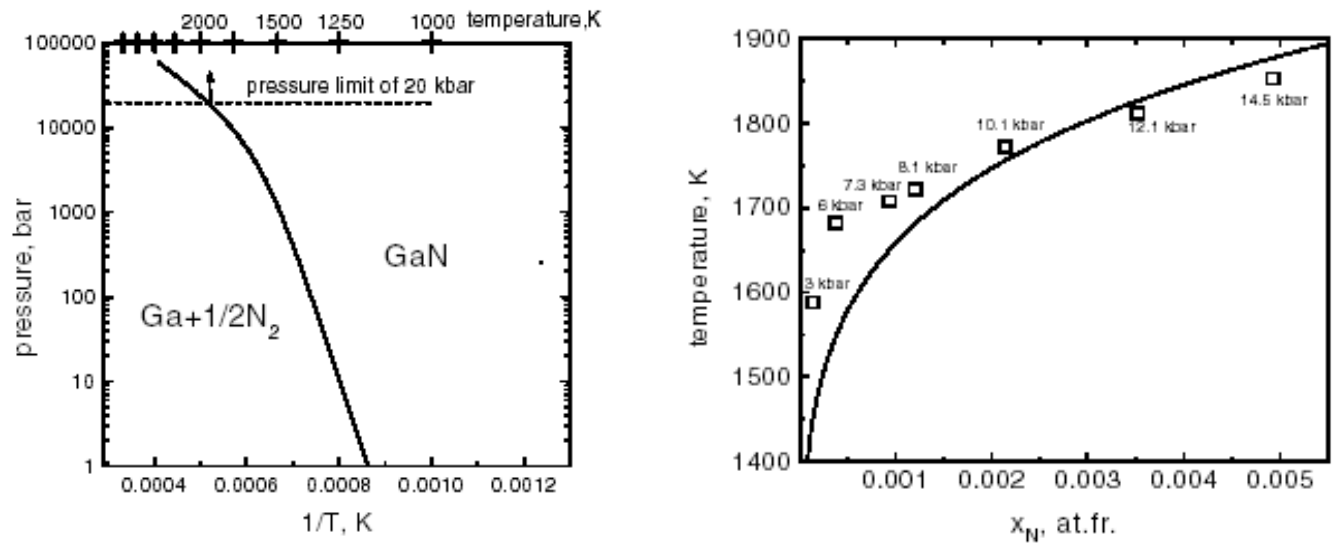

Figure 2.11 Equilibrium curves of $\mathrm{Ga}_{-} \mathrm{N}_{2}-\mathrm{GaN}$ system, adapted from [Karpinski, et al., 1984].

The mechanism of the solution growth is that the nitrogen dissolves into the liquid gallium first and supersaturates the gallium, and then the solid GaN precipitates out of the solution and grows into big crystal. It was found that molecular nitrogen disassociate into atomic nitrogen at the gallium surface [Krukowski, et al., 1999]. The growth of the crystal is anisotropic and low supersaturation favors plate-like crystals growth, while high supersaturation leads to needle-like habits. [Porowski \& Grzegory, 1997; Prywer \& Krukowski, 1998] The largest GaN single crystal achieved by this technique was $17 \mathrm{~mm}$ [Porowski \& Grzegory, 1997] and the dislocation density was found to be expectedly low. Homo-epitaxy on these GaN single crystals also produced very high quality GaN [Zauner, et al., 2002]. The problem with this technique is that the small working volume associated with the harsh conditions makes scale up very difficult. The growth rate in this process is about $0.1 \mathrm{~mm} / \mathrm{h}$, which is moderate. 
To reduce the harsh conditions in high pressure solution growth, some researchers added some alkaline metals such as $\mathrm{Na}$ [Yamane, et al., 1998], K [Yamane, et al., 2002], Li [Song, et al., 2004] to the Ga and synthesized gallium nitride single crystals under moderate conditions of $600-800{ }^{\circ} \mathrm{C}, 10-50$ bar. The nitrogen sources are $\mathrm{NaN}_{3}$ [Yano, et al., 2000], $\mathrm{N}_{2}$ [Aoki, et al., 2000] or $\mathrm{NH}_{3}$ [Iwahashi, et al., 2003]. Both hexagonal [Yamane, et al., 1998] and cubic [Yamane, et al., 2000] GaN has been synthesized.

The growth mechanisms of this process using alkaline metal are still not clear. It is suggested that alkaline metal might help the initiation of $\mathrm{GaN}$ formation [Yamane, et al., 1997]. The crystal size is still small (several $\mathrm{mm}$ ) due to uncontrolled nucleation and growth.

\subsubsection{Physical Vapor Transport Technique}

Since $\mathrm{N}_{2}$ is not an active source to react with $\mathrm{Ga}$ under normal furnace temperature, more reactive $\mathrm{NH}_{3}$ is used instead for synthesizing GaN. Different processes such as $\mathrm{NH}_{3}$ flowing over molten gallium [Ejder, 1974], reaction of gallium vapor with $\mathrm{NH}_{3}$ [Elwell, 1984], sublimation of GaN power [Kamler, et al., 2000)] had been tried to achieve free standing bulk GaN crystals. The growth takes place at 800$1200{ }^{\circ} \mathrm{C}$ and the pressure varies from tens of torr to atmosphere pressure. Of all these processes, vapor transport of $\mathrm{Ga}$ source to cooler region and reacts with $\mathrm{NH}_{3}$ shows most promising since it is very simple and growth rate of $1 \mathrm{~mm} / \mathrm{h}$ had been demonstrated [Baranov, et al., 1998].

The reaction between $\mathrm{NH}_{3}$ and gallium is rather complex. The initial stage of growth occurs at the wall of the crucible or on the substrates. Further growth includes the absorption and desorption of gallium and nitrogen source, surface diffusion and reaction. 
The nucleation can be controlled using GaN single crystal seeds [Shin, 2002] or single crystal substrates [Balkas, 2000]. The factors affecting the growth include pressure, temperature, V/III ratio, distance between source and growth zone, carrier gas, etc.

Comparing with AlN growth using vapor transport, GaN growth is more difficult since GaN start to decompose above $800{ }^{\circ} \mathrm{C}$ and $\mathrm{Ga}$ accumulates to form droplet on the surface [Koleske, 2001; L'vov, 2000]. In order to achieve larger crystal, more understanding and optimization of the process has to be done.

\subsubsection{Ammonothermal Growth}

Supercritical fluids have been widely used in industries for different processes. One example is the synthesis of single crystal quartz in supercritical water [Hosaka \& Taki, 1981]. The principle of ammonothermal growth of GaN is that the mineralizers attack the feedstock to form soluble species in supercritical $\mathrm{NH}_{3}$ that can transport through the fluid to crystallize at the cooler region. The process for $\mathrm{GaN}$ is expressed as [Ketchum \& Kolis, 2001]:

$\mathrm{GaN}+\mathrm{NH}_{2}^{-}+3 \mathrm{NH}_{3} \rightarrow$ soluble Ga complex $\rightarrow \mathrm{GaN}$ crystals $+\mathrm{NH}_{2}^{-}+3 \mathrm{NH}_{3}$

The pressure of this process ranges from 2400-5000 bar and the temperature is usually from 400 to $600{ }^{\circ} \mathrm{C}$. The feedstock includes GaN, gallium imide [Jouet, et al., 2002], $\mathrm{GaI}_{3}$ [Purdy, et al., 2003]. $\mathrm{KNH}_{2}$ \& KI [Ketchum \& Kolis, 2001], $\mathrm{LiNH}_{2}$ [Cumberland, R.W., et al., 2001], $\mathrm{KN}_{3}$ [Raghothamachar, et al., 2002], $\mathrm{NH}_{4} \mathrm{X}(\mathrm{X}=\mathrm{Cl}$, Br, I) [Purdy, et al., 2002], have been used as the mineralizers. Both cubic [Purdy, A.P., 1999] and hexagonal GaN have been synthesized.

The achievement of this process still lags behind the high pressure solution growth and vapor transport methods. The growth rate is relatively slow, less than $5 \mu \mathrm{m} / \mathrm{h}$. 


\subsubsection{Low Pressure Approach}

Activated nitrogen species such as $\mathrm{N}^{-} \mathrm{N}^{-}, \mathrm{N}_{2}^{+}$in plasma could dissolve and saturate gallium at low pressure since they are more energetic and require much lower equilibrium pressure over gallium. It was calculated that the equilibrium pressure of atomic nitrogen over $\mathrm{GaN}$ is $1.5 \times 10^{-7}$ bar at $2000 \mathrm{~K}$ [Argoitia, A. \& Angus, J.C., et al., 1997], compared with the high equilibrium pressure of $\mathrm{N}_{2}$ over GaN (20000 bar at 2000 K [Karpinski, et al., 1984]. Atomic nitrogen dissolves into and supersaturates liquid gallium and $\mathrm{GaN}$ crystals precipitate out, but at the same time $\mathrm{GaN}$ decomposes and release $\mathrm{N}_{2}$ since the $\mathrm{N}_{2}$ pressure is far less the equilibrium pressure.

Bulk nucleation and growth of polycrystalline GaN crystals from gallium melt exposed to nitrogen plasma has been demonstrated [Argoitia, et al., Angus, 1997]. The conditions were at $1000{ }^{\circ} \mathrm{C}, 0.5$ mtorr and the growth rate was estimated to be $8 \mu \mathrm{m} / \mathrm{h}$. However the spontaneous nucleation during this process only results in only micron size polycrystals.

\subsection{Summary}

In summary, there is a tremendous need for process development to grow large area, low defect density $\left(<10^{6} \mathrm{~cm}^{2}\right)$, single crystal $\mathrm{GaN}$ for achieving the full potential of III-nitrides. Bulk crystal growth techniques are promising but are presently limited to growth of smaller sizes of crystals. So, new growth processes based on bulk growth techniques are critically needed. 


\section{CHAPTER 3}

\section{PROPOSED CONCEPTS FOR GALLIUM NITRIDE CRYSTAL GROWTH AND}

\section{NANOWIRE SYNTHESIS}

\subsection{GaN Formation Using Nitrogen Plasma at Sub-atmospheric Pressures}

Nitrogen plasma which contains active nitrogen species such as $\mathrm{N}, \mathrm{N}^{-}, \mathrm{N}_{2}^{+}$and $\mathrm{N}_{2}{ }^{*}$ could react with gallium under moderate temperatures to form GaN [Argoitia, A. \& Angus, J.C., et al., 1997]. For simplicity, the process can be written as:

$$
\mathrm{Ga}(\mathrm{l})+\mathrm{N}(\mathrm{g}) \leftrightarrow \mathrm{GaN}(\mathrm{s})
$$

Thermodynamically, the Gibbs free energy of the above reaction is

$$
\Delta \mathrm{G}=-392.561 \mathrm{KJ} / \mathrm{mol}<0
$$

for a reaction temperature of $850^{\circ} \mathrm{C}$, which means that the reaction is spontaneous. The equilibrium pressure of atomic nitrogen was calculated to be around $1.5 \times 10^{-14}$ bar at 1370 K using thermodynamic data from reference [Knacke, et al, 1991] (See Appendix III). The partial pressure of atomic nitrogen in our system with total pressure of 50 mtorr, 150 $\mathrm{W}$ microwave power is calculated to be on the order of $10^{-6}$ bar using SAMPR software [Licensed software from Dr. M. Meyappan, NASA-Ames Research Center], which is much higher than the equilibrium pressure of atomic nitrogen over GaN. At the same time, the GaN decomposes since the pressure of $\mathrm{N}_{2}$ is far below the equilibrium pressure, but this decomposition process is slow and GaN crystal growth is possible and had been demonstrated [Argoitia, A. \& Angus, J.C., et al., 1997]. 
The kinetics of $\mathrm{GaN}$ formation includes dissolution of atomic nitrogen into molten gallium, supersaturation of molten gallium, nucleation and growth of $\mathrm{GaN}$ crystals. The formation of $\mathrm{GaN}$ with direct reaction between $\mathrm{Ga}$ and atomic nitrogen has also been confirmed by the study under high pressure where molecular nitrogen absorbs onto gallium and dissociates into nitrogen atoms [Krukowski, et al., 1998]. The diffusion process is not clear about whether the atomic nitrogen diffuses along or by means of cluster formation [Klemenz \& Scheel, 1998].

\subsection{Proposed Concept of Self-oriented GaN Growth on Molten Gallium}

The technique of growing GaN crystals using plasma usually results in only micron size poly crystals due to the spontaneous nucleation. However, one interesting phenomena that nitrogen dissolved gallium melts wet solid surfaces very well had been discovered by several researchers [Madar, R., et al., 1975; Elwell, D., et al., 1984]. It is also found that the gold nuclei and islands could coalesce like a liquid when they touch one another during growth on a solid substrate and the slightly misaligned nuclei tended to rotate slightly during growth into perfect alignment [Pashley, D.W., et al., 1964]. The driving force for these occurrences is believed to be the minimum interfacial energy [Pashley, D.W., 1965]. Our idea for this study is to utilize the wetting phenomena to allow growing GaN platelets crystals on top of molten gallium to self-orient with respect to each other during nucleation and growth. These GaN crystals would join together during growth to form large area single crystals since their mobility on top of molten liquid gallium is better than that on solid substrates. The proposed self-oriented growth concept is schematically illustrated in Fig. 3.1. The dislocations which are common in vapor phase grown $\mathrm{GaN}$ are not expected for the individual $\mathrm{GaN}$ crystals as they are 
grown in a Qausi-bulk fashion directly from molten gallium. The self-alignment could also be externally favored using applied forces such as substrate rotation.
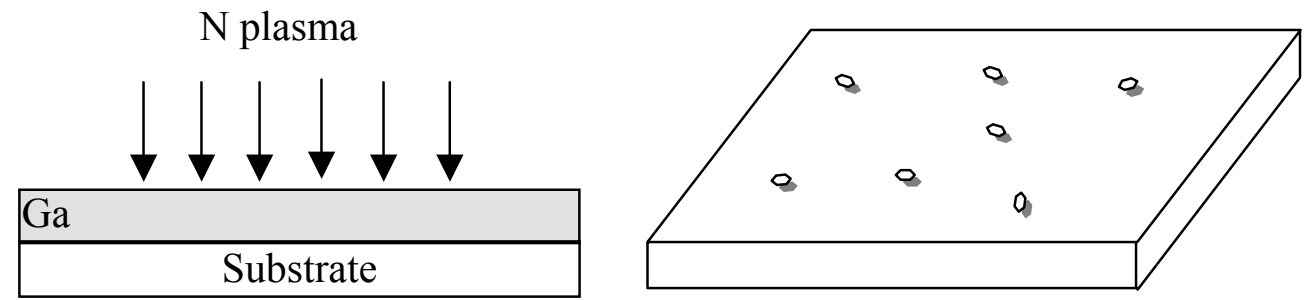

Nucleation
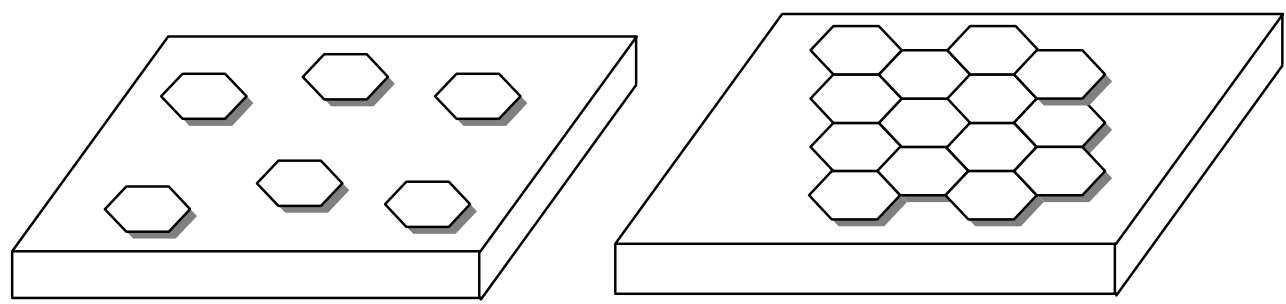

Growth \& Alignment

Joining

Figure 3.1 Sketch of self-oriented growth of GaN platelets on molten gallium to form large area GaN crystal.

With the growth of GaN platelets crystals followed by joining together to form large area $\mathrm{GaN}$ films, further dissolution of atomic nitrogen into gallium has to diffuse through the GaN layer, which is becoming more and more difficult. The thickness of $\mathrm{GaN}$ film is expected to approach a limit. To produce thicker GaN crystals, homo-epitaxy onto these self-oriented GaN film could be conducted.

\subsection{Proposed Concept for GaN Nanowires Synthesis with Control on Growth Directions}

Spontaneous nucleation and growth of GaN from molten gallium under wetting conditions promote 2-D crystal growth. Change of gas phase compositions could change 
the wetting properties of gallium and result in 1-D growth [Sharma \& Sunkara, 2002].

The anisotropic nature of wurtzitic $\mathrm{GaN}$ is hypothesized here to facilitate the growth of 1D structures with different directions under appropriate conditions.

\subsection{Proposed Concept of Homo-epitaxy onto Long GaN Nanowires for Large Single Crystal Growth}

Nanowires have been well known for their single crystalline structure, native with no dislocations and stacking faults [Sunkara, M. K., et al., 2001; Huang, Y., et al. 2002]. These nanowires could be as long as several hundreds microns [He, M., et al. 2001]. Therefore we proposed a concept of using ultralong nanowires similar to that of the carbon nanotubes [Huang, S.M., et al. 2003] as a seed for growing large area single crystals by homo-epitaxy. Therefore it is very important to synthesize long and defects free nanowires with different directions. 


\section{CHAPTER 4}

\section{EXPERIMENTAL}

\subsection{Introduction}

This chapter gives the typical nitridation procedures and the materials characterization techniques for understanding the composition, quality and orientation of the resulting oriented $\mathrm{GaN}$. The experiments were performed in a vacuum chamber using a substrate heater for controlling temperatures. The active nitrogen is provided though a high density electron cyclotron resonance microwave (ECR-MW) generated plasma. The Scanning Electron Microscopy (SEM) is used for morphology characterization. The orientation of the $\mathrm{GaN}$ films is extensively studied through X-ray diffraction (XRD) and convergent beam electron diffraction (CBED). The texture analysis using XRD is used to determine the overall orientation information about the GaN films and the Kikuchi lines analysis in CBED patterns is used to determine the orientation between individual grains. The quality of the GaN is characterized by Raman spectroscopy and photoluminescence (PL).

\subsection{Nitridation Experiments}

\subsubsection{ECR-MW Plasma Reactor}

The experiments were conducted using an 8-inch diameter, water-cooled double wall stainless steel vacuum chamber with an $\mathrm{ASTeX}^{\circledR}$ Model AX4500 electron cyclotron resonance (ECR) plasma source. The plasma source is connected to the vacuum chamber with a standard ISO-NW160 flange. A sketch of the system used for the GaN synthesis is 
shown in Fig. 4.1. The nitrogen plasma diffuses from the ECR source down to the substrates heated by a ceramic heater. The substrates were placed at the same height of the view-port of the chamber so the observation of growth process is possible. The distance between the gas inlet and the substrates is about 10 inches. The flow rate is controlled by MKS model 1179 mass flow controller (MFC). The chamber is connected with both a mechanical pump (Alcatel 2033C2, capacity $23.3 \mathrm{cfm}$ ) and a turbo pump (Alcatel 5080, capacity $80 \mathrm{l} / \mathrm{s}$ ). The pressure is measured with two different pressure sensors with different range. A HPS Pirani convection pressure gauge measures pressures up to 760 torr. A MKS Baratron type 627 pressure transducer has a range of 0.1 mtorr to 1 torr.

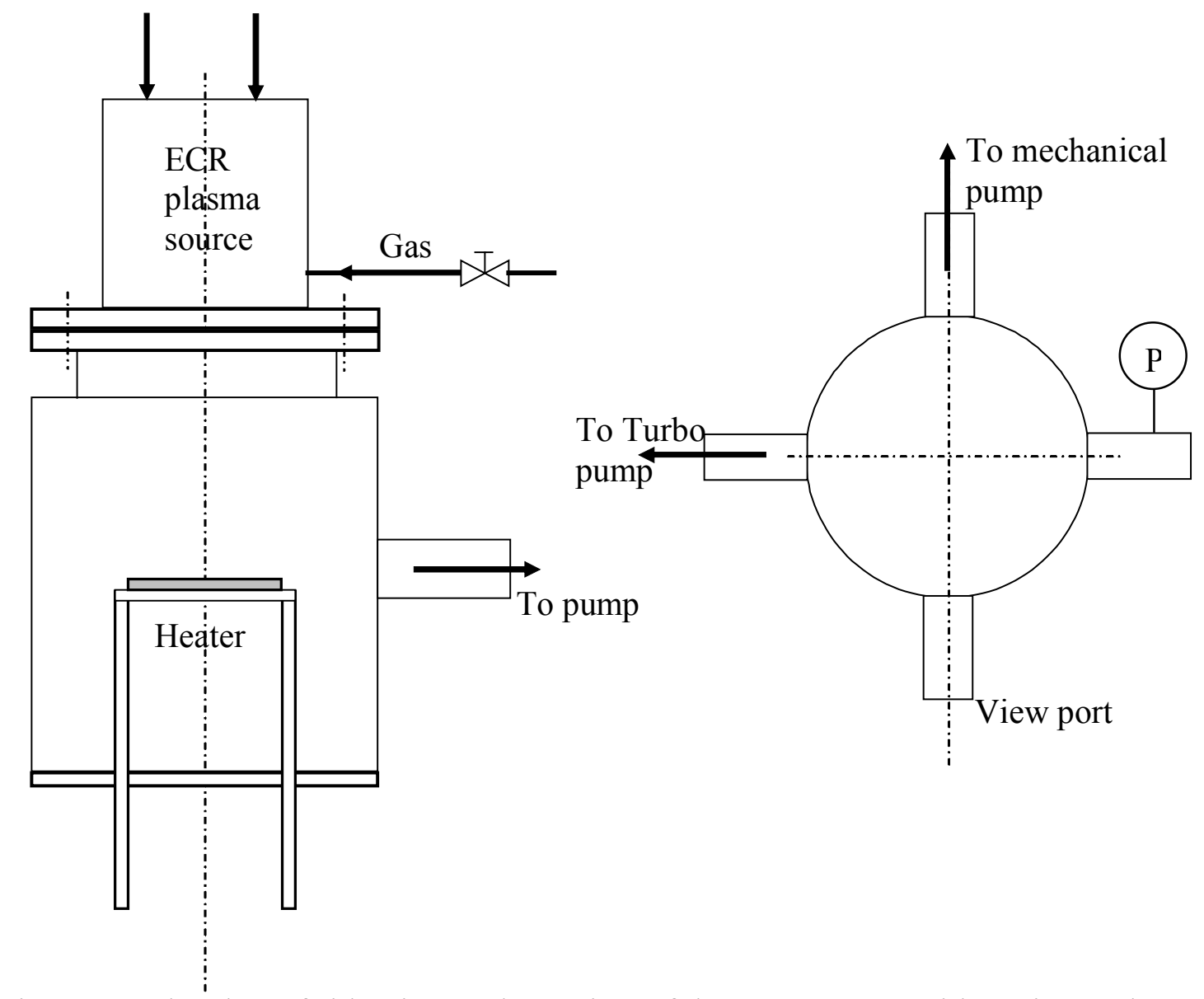

Figure 4.1 Sketches of side-view and top-view of the ECR reactor with stationary heater. 
The ASTeX ${ }^{\circledR}$ plasma source is a high density plasma source which operates at low pressures. The system consists of the AX4500 ECR plasma source, S250 microwave power generator, isolator, coaxial slugger tuner, rigid coaxial microwave transmission cable, a magnet power supply and magnet power cable. The configuration of the system is shown in Fig. 4.2. The plasma chamber, the isolator and the magnet are cooled with cooling water for proper operation.

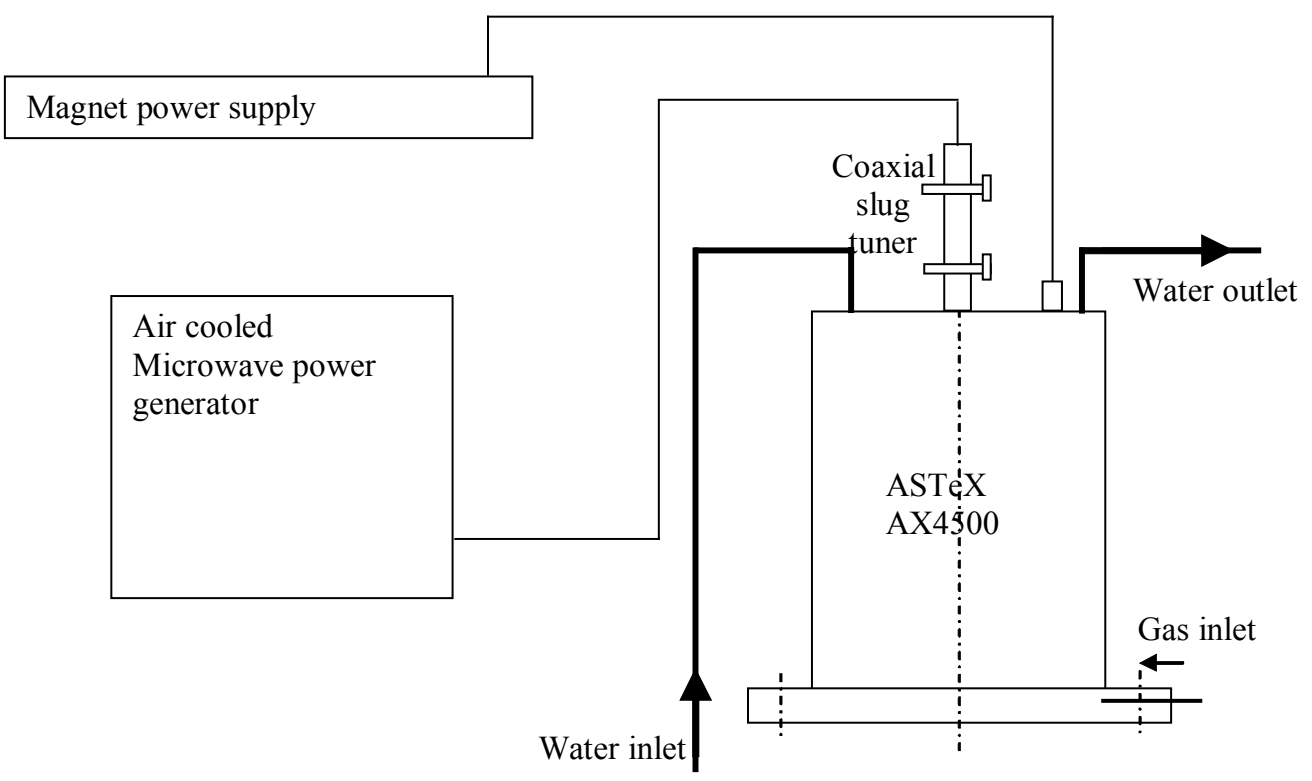

Figure 4.2 Sketch of ECR-MW systems.

The plasma in the Electron Cyclotron Resonance is confined in a quartz bell jar surrounded by the radial magnetic field produced by the magnet. The microwaves with a frequency of $2.45 \mathrm{GHz}$ from the microwave generator enters into the quartz bell jar through the cable and ionizes the gas to form a plasma. The electrons gyrate around the magnetic line due to Lorentz force with a gyration frequency called the cyclotron frequency $\omega_{\text {cyc }}$. When the microwave radiation of the same frequency propagates into this 
region, microwave energy is efficiently absorbed by these electrons and they are resonantly accelerated when the electron cyclotron resonance condition is fulfilled:

$$
\omega_{\mathrm{mv}}=\omega_{\mathrm{cyc}}=(\mathrm{e} / \mathrm{m}) \cdot \mathrm{B}
$$

Where e and $\mathrm{m}$ denote the charge and mass of the electron, respectively. B is the intensity of the magnetic field, which is 875 Gauss. (Comparing with magnetic intensity near the surface of Earth of 0.3-0.5 gauss) These electrons gain high energies and ionize plasma atoms, gas molecules, and ions into high charge states via continuous collision. Therefore the density of the plasma is greatly enhanced.

\subsubsection{Substrate Heater Set-Up}

The heating elements of the system are provided through two electrode feedthroughs from the base plate. The heaters are connected using copper and molybdenum connections. Different heaters such as crucible heaters, flat ceramic heaters with different height could be installed with maximum flexibility. Most of the experiments were done using a pyrolytic boron nitride $(\mathrm{pBN})$ heater (Model GE Advanced ceramics, HTR1002) which has a very uniform temperature distribution on the

whole substrates. The temperature was measured using C-type thermocouple inserted into the holes of the ceramic heater. Calibration of the temperature of the substrates on both sides has also been conducted for each set-up before the experiments. 

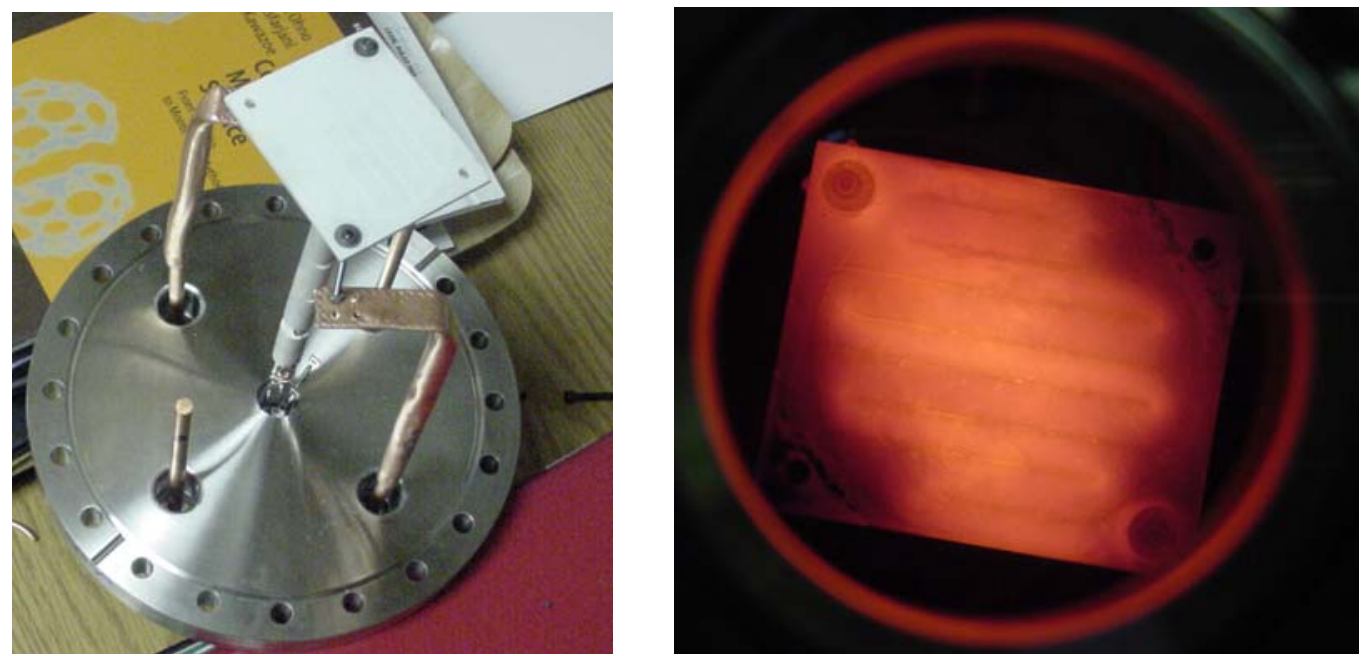

Figure 4.3 Pyrolytic boron nitride (pBN) heating set-up (a) Ceramic heater assembly (b) Heater under high temperature.

\subsection{Typical Nitridation Procedures}

Fused amorphous quartz substrates were cleaned by using hot $\mathrm{KOH}$ and acetone and then blown dry with argon. A layer of gallium was spread onto the substrates by hand. The chamber was pumped to base pressure and purged with $\mathrm{N}_{2}$ for several times to remove any possible residual oxygen. $\mathrm{H}_{2}$ plasma at room temperature was used for about 30 minutes to remove possible oxide on the surface of gallium. Subsequently, the gallium film was exposed to $\mathrm{N}_{2}$ plasma and heated to $900-1000{ }^{\circ} \mathrm{C}$ for $3-5$ hours at a pressure of 60 mTorr. The shutdown process was performed by first lowering the temperature down to $500{ }^{\circ} \mathrm{C}$ in 30 minutes with $\mathrm{N}_{2}$ plasma on, followed by turning off plasma and keeping flowing pure $\mathrm{N}_{2}$. Finally, the substrate was cooled down further under flowing nitrogen to room temperature.

The excess gallium is removed using aqua regia (a mixture of nitric and hydrochloric acids with a 1:3 volume ratio) if needed to get free standing GaN crystals. 
The samples were broken into small pieces and were characterized using different techniques.

\subsection{Materials Characterization Techniques}

The synthesized self-oriented gallium nitride films were characterized using scanning electron microscopy (SEM, model ZEISS SUPRA ${ }^{\text {TM }}$ 35VP), X-ray Diffraction (XRD, model Rigaku VS-DRX3000), transmission electron microscopy (TEM, model JEOL2000 \& 2010), Raman spectroscopy and photoluminescence (model Renishaw Invia) to study their structural and optical properties. Scanning electron microscopy is one of the main instruments for studying the morphologies of the GaN crystals and the details about the principles of SEM are referred to the textbook [Goldstein, J.I., et al. 1992]. The orientations of the large area GaN films were investigated using X-ray texture analysis to obtain the overall orientation information. To study the orientation relationship between individual crystals and the development of grain boundaries during growth, transmission electron microscopy (TEM) analysis of samples including both plain view and cross sectional samples were conducted. The optical properties of the GaN crystals were studied using Raman spectroscopy and photoluminescence (PL).

\subsubsection{X-ray Diffraction}

X-ray diffraction is a widely used technique to obtain the information of the structure, orientation, composition, and quality of crystalline materials. A beam of monochromatic X-ray with wavelength comparable to the size of the atoms is directed at a crystalline material, the scattered X-rays will reinforce with each other when it encounters a periodic array of atoms with the incident beam at certain angles. The relationship between the wavelength of X-ray beam $\lambda$, the angle between incident X-ray 
and particular crystal planes $\theta$, the distance between atomic planes of the crystal d, is given as Bragg law proposed by W. L. Bragg:

$$
\mathrm{n} \lambda=2 \mathrm{~d} \sin \theta
$$

where $\mathrm{n}$ is integer, represents the order of diffraction. Each crystalline material has a unique diffraction pattern which can be treated as "figure print" of the materials.

\subsubsection{Single Crystal Diffraction}

Powder diffraction technique is the main approach to differentiate different crystalline materials by illuminating a large number of randomly oriented small crystallites. The spectra plot the intensity of the diffracted beam with respect to the diffraction angle (angle between transmitted and diffracted beam) 20. All allowed peaks show up with intensities at certain ratios for polycrystalline materials. Reflection mode shown in Fig. 4.4 is commonly used in the powder diffractometer. However, for single crystal diffraction, only certain peaks relevant to the orientation of the crystals appear.

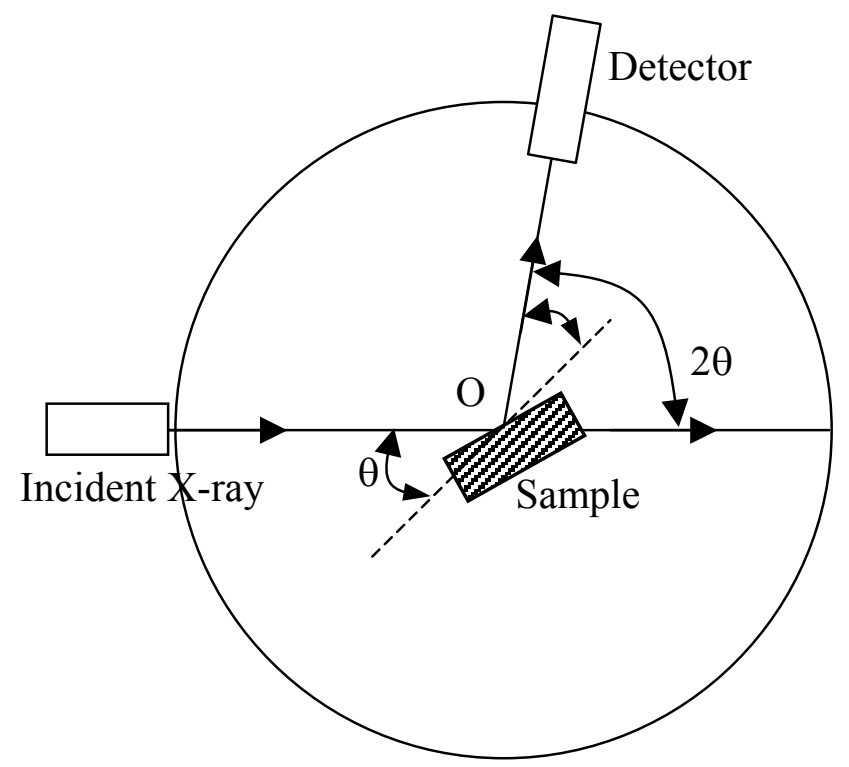

Figure 4.4 Configuration of powder XRD setup. 
Rocking curve analysis has been the main method to determine the quality of a single crystal. Contrary to the set-up for powder X-ray diffraction, the detector is set at a known Bragg angle $2 \theta$ and the crystal sample is rotated around angle $\theta$ in a rocking fashion. The full width at half maximum (FWHM) of the rocking curve is a direct measurement of the quality of the single crystal. Theoretically, the FWHM of rocking curves for a perfect crystal with no curvature could be as small as $10 \operatorname{arcsec}\left(1^{\circ}=3600\right.$ arcsec) [Cullity \& Stock, 2001]. Epitaxially grown GaN on sapphire substrates usually has a FWHM of 125 to 700 arcsec [Reifsnider, J.M. et al., 1998], while bulk grown single GaN crystal from gallium melts has shown FWHM of a rocking curve as small as $22 \operatorname{arcsec}$ [Lefeld-Sosnowska \& Frymark, 2001].

\subsubsection{Texture Analysis}

Texture, or preferred orientation, is termed for a polycrystalline sample whose grains are aligned in particular orientation or orientations. The preferred orientation has a dominant effect on the intensities of the diffracted peaks in the XRD spectra.

In thin film materials, the preferred orientation can be caused by both initial nucleation and later growth processes. The initial orientations of nuclei could be due to epitaxy on a single crystal substrate [Herres, N., et al., 1999], evolutionary selective growth on a randomly oriented polycrystalline substrate [Noda, S., et al., 2004], preferred formation of certain planes parallel to amorphous substrates [Hayamizua, S., 1996]. The orientation may also be established during growth due to the different growth rates of certain orientations [Novikov, V.Y., 1999] as well as recrystallization [Kaminski, E. \& Ribe, N.M., 2001]. Several models have been developed to explain and simulate the development of texture growth in thin films [van der Drift, A., 1967; Karpenko, O. P., 
Bilello, J. C. \& Yalisove, S. M., 1997].

The preferred orientation can be determined by studying either the orientation of selected crystal planes or the complete orientation of individual grains in the sample. It can also be studied statistically by measuring the diffraction of large volume of grains. Xray back reflection texture diffractometer (Bruker-AXS General Area Detector Diffraction System (GADDS)) shown in Fig. 4.5 is used in the experiments to determine the texture of the oriented GaN crystals. Scans are taken using the area detector by varying three different angles, which $2 \theta$ represents the Bragg angle of certain planes of the crystal, $\omega$ is rotation around the normal of the sample surface and $\chi$ is the tilt with respect to the plane represented by X-ray source and detector. Both phase and orientation information can be obtained using this type of graphical representation from a single scan.

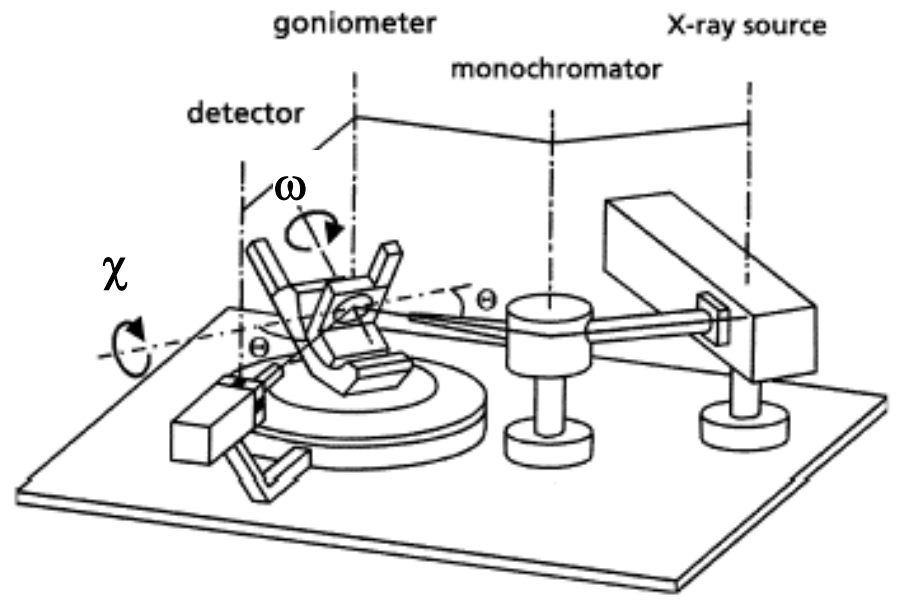

Figure 4.5 X-ray texture diffractometer for determining the orientation of large area oriented GaN crystals, adapted from [Herres, N., et al., 1999]. 


\subsubsection{Transmission Electron Microscopy}

TEM is a powerful tool to study the structure and morphologies of the materials by studying the transmitted and diffracted electron beams. The small wavelength of electrons makes it possible to get a resolution of sub-nanometer. The state-of-art of high

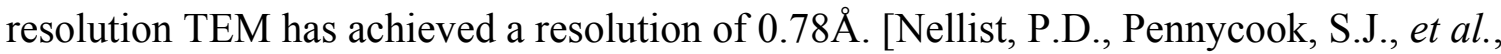
2004] Electron diffraction is quite different from X-ray diffraction since electrons are scattered more strongly by both the positively charged nucleus and negatively charged electrons in the sample. Also high index planes are readily diffracted due to the much smaller wavelength of electrons. Most importantly, the electron beams are easy to be directed and selective studies of diffracted beams are possible.

Electron diffraction from crystalline materials provides copious information about the defects, orientation as well as structure. Lattice images in HRTEM can also give details about the defects and growth orientations.

\subsubsection{Convergent Beam Electron Diffraction (CBED)}

In the normal diffraction mode in TEM, the electron beam is parallel when it shines on the sample. The diffraction spots are obtained by selecting a small area under the mode called selected area diffraction (SAD). Another diffraction technique is called convergent beam electron diffraction (CBED) where beam of electrons is converged at certain angle onto the sample.

The area for SAD pattern for general TEM is around $0.5 \mu \mathrm{m}$ compared with beam size of tens of $\mathrm{nm}$ in convergent beam conditions. Therefore CBED pattern can provide a variety of information from a miniscule region which is impossible for other techniques. 
For example, the sample thickness can be determined using CBED pattern and the lattice parameter can be precisely measured [Williams, D.B. \& Carter, C.B., 1996]. Also Kikuchi lines are readily seen in CBED patterns.

\subsubsection{Kikuchi Lines}

More features are seen in addition to the usual Bragg diffraction spots in a little thick TEM samples. The inelastic scattered electrons provide a diffuse beam to form additional information in the diffraction pattern. For example, sets of straight lines called Kikuchi lines appear in the diffraction images. They are the results of elastic Bragg diffraction of the inelastic scattered electron beam. (Fig. 4.6(b)) The inelastically scattered beams that are oriented properly for Bragg diffraction conditions by the crystal planes (hkl) are diffracted by the atoms in the planes. The lines from the beam which are close to the incident direction appears bright (the excel lines) and the other is dark (the deficit lines). The reason of appearing as lines but not spots is because the electron are inelastically scattered in all directions. The Kikuchi lines are very sensitive to the orientation of the specimen and can be used to determine the misorientation with an accuracy of $0.1^{\circ}$ [Wang, S.C. \& Starink, M.J., 2003]. 


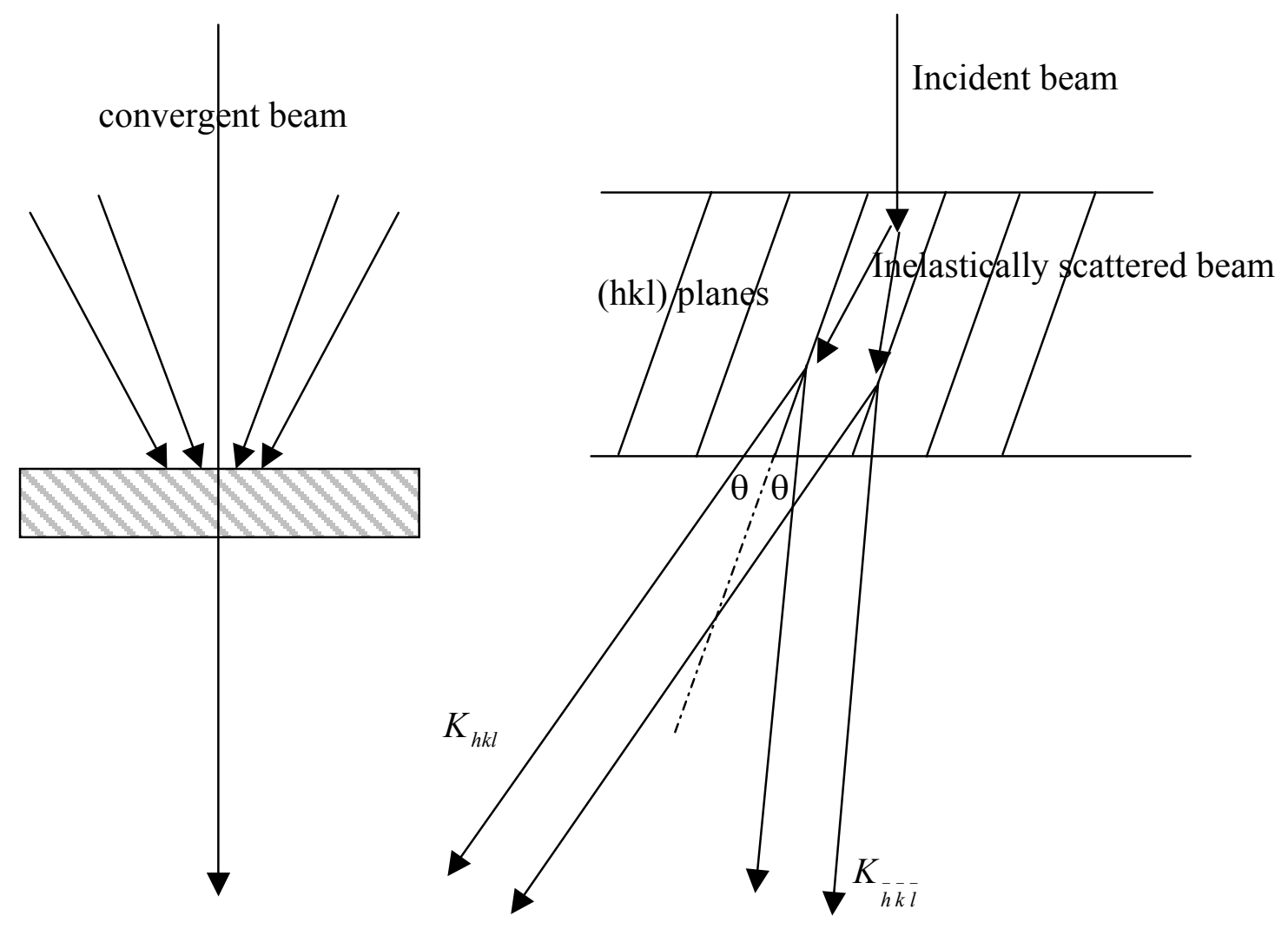

Figure 4.6 (a) Convergent beam mode (b) Sketch of ray diagram for Kikuchi lines in TEM.

Under convergent beam condition, the diffraction patterns always contain sharp Kikuchi lines since the sampling area is much smaller. The zero order Laue zone (ZOLZ) Kikuchi lines in CBED pattern in a zone axis (ZA) appear as bright bands. The elastic scattering in a CBED pattern also contribute to the Kikuchi lines when convergent angle $\alpha$ is bigger than the Bragg angle. As a result, strong Kikuchi lines can be obtained even with a relatively thin sample under convergent beam mode.

\subsubsection{Scanning Transmission Electron Microscopy (STEM)}

When the incident electron beam is allowed to deflect and the transmitted beam is detected by a scintillator rather than a photographic plate, a STEM image can be obtained 
on a CRT. The emission resulting from interaction of the electrons with specimen provides structural and chemical information of the sample. A resolution of few nanometers can be achieved for chemical mapping.

\subsubsection{Sample Preparation}

The sample for the TEM is limited to less than $3 \mathrm{~mm}$ in diameter and less than 0.2 $\mu \mathrm{m}$ in thickness. Sample preparation depends on the specific materials and what kinds of information need to be obtained. It might take several minutes for nanomaterials and several days for cross sectional bulk materials.

Many techniques have been reported for preparing cross section sample for TEM [Chu, S., Sheng, T.T., 1984; Ishitani, T., et al., 1994; McCaffrey, J.P., Barna, A., 1997]. For the GaN flakes samples, it was first sandwiched by two dummy silicon pieces using high temperature glue, then it was cut into a $3 \mathrm{~mm}$ rod using ultrasonic cutter and put into a copper tube with epoxy. The tube is cut into small disks with thickness less than $1 \mathrm{~mm}$. The specimen is then thinned sequentially using mechanical polishing, dimpling the center of the disk, ion milling to electron transparency under liquid nitrogen temperature. Caution has to be taken all the time during thinning process to keep the sample intact. The plan view samples were prepared just by sticking the GaN flakes and ion-milled to electron transparency. The available instruments for the sample preparation are: Buehler Isomet $^{\mathrm{TM}}$ low speed diamond saw, Buhler Ecomet ${ }^{\mathrm{TM}}$ grinder/polisher, Fischione Model 170 ultrasonic disk cutter, Fischione Model 160 specimen grinder, Fischione Model 150 Dimpling Grinder, Fischione Model 180 cross section TEM preparation kit, Fischione Model 1010 low angle milling system. 


\subsubsection{Raman Spectroscopy}

Raman spectroscopy is a nondestructive characterization technique to determine the intermolecular and interatomic bonds, mechanical strain in a solid, the crystal structure and degree of crystallinity of a solid, composition of materials qualitatively and quantitatively. The sample preparation is simple and the resolution can reach several microns with an optical microscope. The limitation of Raman spectroscopy is that the samples might have minor component whose fluorescence excited by the laser radiation could be sufficiently intense to mask the Raman effect.

When a monochromatic light shines on the material, there are two different scattering phenomena. One is known as Raleigh scattering, i.e., the scattered light have the same wavelength as the incoming light. The other type of scattering is that of Raman effect in which the wavelengths of scattered light are shifted from the incident light. Raman spectrum plots the intensity of the scattered light as a function of the shift in the wave number of the radiation and the peak intensities are proportional to the number of scattering molecules in the path of the light.

\subsubsection{Luminescence}

\subsubsection{Photoluminescence}

Photoluminescence (PL) spectroscopy is a powerful technique to study the electronic structure of semiconductors. PL collected at liquid helium temperature $(2 \mathrm{~K})$ has excellent information about overall crystal quality and purity. It can be used to measure the bandgap of semiconductors and identify the defects. The penetration depth for PL is usually on the order of one micron. 
When a light with photon energy greater than the band-gap energy of the semiconductor materials, the electrons from valence band are excited to the conduction band, leaving holes in the valence band. The electrons in the conduction band are not at stable state and recombine with the holes in the valence band with emitting a photon that has energy characteristic of the material and the particular radiative process. The electrons also might fall into a defect state by nonradiative recombination and then undergoes radiative recombination which results in emission of longer wavelength photons. Spectra from transition involving defects are usually broad since these defects states are localized. At room temperature, there is a nonzero occupancy of electron states near the bottom of the conduction band and holes states near the top of the valence band. Therefore, the peak is broad since the electron-hole pairs recombine and emit photons over a range of energy.

\subsubsection{Cathodoluminescence}

Cathodo luminescence is the emission of light from a material under electron beam. The process involves generation and recombination of electron-hole pairs and the spectrum is collected as the intensity of emitted light versus the wavelength. Therefore it is a powerful tool in the characterization of semiconductors.

\subsubsection{Time-resolved Cathodoluminescence}

The normal luminescence measurements using a continuous-wave (CW) light source only gives time-averaged information of the carriers, whereas a time-resolved luminescence could reveal not only time-integrated information but also the dynamical processes of the carriers in semiconductor materials. For example, the recombination rate of various transitions, exciton binding energy and quantum efficiency can be obtained 
from the time-resolved luminescence system. Therefore, a very high time resolution which is at least 10 times shorter than the studied optical transitions is necessary.

A time-resolved system usually includes a fast-pulsed light source for excitation, a monochromator for separating the emitted photons and a fast signal detector. The light source is electron burst from chevron micro-channel-plates (MCP) for time-resolved cathodoluminescence. The photon counting setup is configured around "HARP", a photon counting system of PicoQuant GMBH. The specific configuration of the system for time-resolved cathodoluminescence is referred in Appendix I.

\subsection{Summary}

A combination of characterization techniques was used to study the composition, morphology, quality and orientation of the resulting GaN materials. 


\section{CHAPTER 5}

\section{ORIENTED GALLIUM NITRIDE GROWTH ON MOLTEN GALLIUM}

\subsection{Introduction}

The concept of oriented GaN crystal growth on molten gallium necessitates the proper control of the molten gallium surface as well as the interaction between the platelet-shaped crystals. The wetting properties of gallium under growth conditions are critical for the 2-D crystal growth. The dynamic growth processes of GaN crystals are important for achieving large area oriented GaN films. This chapter presents the fundamental studies of spreading of gallium melts and dynamic growth processes of GaN crystals. The orientation of the resulting films is extensively investigated to understand the growth mechanism and the development of the grain boundaries. The quality of the oriented GaN films is also studied for optimization of the processes.

\subsection{Dynamic Growth Processes of GaN from Molten Ga}

\subsubsection{Self-spreading Phenomena of Nitrogenated Ga}

\subsubsection{Wetting}

A liquid droplet reaches an equilibrium shape when it interacts with a solid surface. This phenomenon is characterized by the contact angle formed at its edge where the liquid contacts the solid surface. (Fig. 5.1) The relationship between the contact angle of the droplet and the interfacial tensions of the three phases was first discovered by the British scientist Thomas Young in 1805 [Young, T., 1805] and expressed as:

$$
\gamma_{S V}-\gamma_{S L}=\gamma_{L V} \cos \theta
$$


Where:

$\gamma_{\mathrm{lv}}$ is the surface tension of the liquid;

$\gamma_{\mathrm{sl}}$ is the interfacial tension between the solid and liquid;

$\gamma_{\mathrm{sv}}$ is the surface tension of the solid;

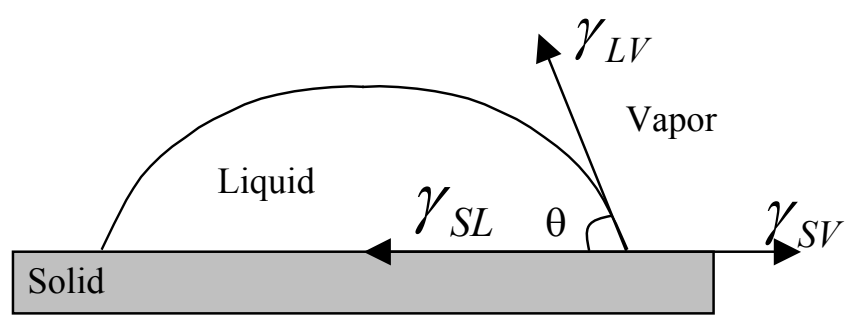

Figure 5.1 Wetting of a liquid on a solid surface.

It can be derived that the same liquid will have smaller contact angle on a surface with high surface free energy. Wetting can be categorized into three regimes based on the contact angle [Murr, L.E., 1974]:

$$
\begin{aligned}
& \theta=0^{\circ} \text { for complete wetting (spreading) } \\
& 0^{\circ}<\theta \leq 90^{\circ} \text { for partial wetting } \\
& 90^{\circ}<\theta \leq 180^{\circ} \text { for non-wetting }
\end{aligned}
$$

Since the contact angle of less than zero is not measurable, therefore a spreading coefficient $\mathrm{S}$ is introduced to characterize the driving force for complete wetting. The coefficient is defined as following:

$$
S_{L-S}=\gamma_{S V}-\gamma_{S L}-\gamma_{L V}
$$

The positive spreading coefficient means spontaneous spreading of liquid over the solid surface, the higher the value, the bigger driving force for spreading. 


\subsubsection{Contact Angle of Ga on GaN}

According to Equation on (5.1), the values for surface free energy of the liquid, the solid and the interfacial energy are necessary to obtain the value of the contact angle. Usually, the surface free energy of liquid is readily available. The surface free energy of solid is not widely known but could be found. The interfacial energy is rarely available. Some models have been proposed to try to calculate the values of the interfacial energy between a solid and a liquid [Warren, R., 1980; Miedema, A.R. \& Broeder, F.J.A., 1979; Coudurier, L., et al., 1978].

According Warren's two atomic layers interface thermodynamic analysis [Warren, R., 1980], the interfacial energy in pseudo-binary system with no liquid dissolving into the solid or low solubility of solid in liquid is given:

$$
\gamma_{s l}=\gamma_{s l}^{\prime}+\gamma_{s l}^{\prime \prime}
$$

Where $\gamma_{s l}{ }^{\prime}$ is the energy of an S-L interface between a solid and a liquid with the same composition; $\gamma_{s l}$ " is the energy of a S-L interface between two structurally similar but chemically different phases.

$$
\begin{gathered}
\gamma_{s l}^{\prime}=\frac{k T_{m}}{b\left(\frac{V}{b}\right)^{\frac{2}{3}}} \\
\gamma_{s l}^{\prime \prime}=\frac{n F}{N} \\
F=H_{m}\left(1-\frac{T}{T_{m}}\right) X^{2}+R T[X \ln X+(1-X) \ln (1-X)]-R T X(1-X) \ln X_{s} \\
X=\frac{n_{l} X_{s}+n_{s}}{n}
\end{gathered}
$$

where $\mathrm{T}_{\mathrm{m}}$ : melting point of the solid $(\mathrm{K})$;

b: number of atoms in the solid molecule; (for $\mathrm{GaN}, \mathrm{b}=2$ ) 
$\mathrm{V}$ : molar volume of the compound solid $\left(\mathrm{m}^{3}\right) ;(\mathrm{GaN})$

$\mathrm{n}$ : number of interface atoms, $\mathrm{n}_{\mathrm{s}}+\mathrm{n}_{1}$;

$\mathrm{k}$ : empirical constant, $5 \times 10^{-4}-8 \times 10^{-4}$;

$\mathrm{H}_{\mathrm{m}}$ : Latent heat of melting of the solid;

$\mathrm{X}_{\mathrm{s}}$ : molar fraction of solid atoms in liquid solution; (solubility of $\mathrm{GaN}$ in $\mathrm{Ga}$ equals to $\mathrm{N}$ solubility in $\mathrm{Ga}$ )

N: Avogadro's number;

$n_{S}=\left(\frac{N}{V_{s}}\right)^{\frac{2}{3}} ; \quad \mathrm{V}_{\mathrm{s}}$ is the average molar volume of the atoms in the solid $\left(\mathrm{m}^{3} / \mathrm{mol}\right) ;$

$V_{S}=\frac{W_{S}}{\rho_{S}} ; \mathrm{W}_{\mathrm{s}}$ is the atomic weight $(\mathrm{g} / \mathrm{mol}), \rho_{\mathrm{s}}$ is the density $\left(\mathrm{g} / \mathrm{m}^{3}\right)$;

$n_{l}=\left(\frac{N}{V_{l}}\right)^{\frac{2}{3}} ; \mathrm{V}_{1}$ is the average molar volume of the atoms in the liquid $\left(\mathrm{m}^{3} / \mathrm{mol}\right)$;

$V_{l}=\frac{W_{l}}{\rho_{l}} ; \mathrm{W}_{1}$ is the atomic weight $(\mathrm{g} / \mathrm{mol}), \rho_{1}$ is the density $\left(\mathrm{g} / \mathrm{m}^{3}\right)$;

For $\mathrm{GaN}-\mathrm{Ga}$ binary system, the melting point of $\mathrm{GaN}$ is taken as $2500^{\circ} \mathrm{C}$ [ Van Vechten, 1973], the temperature under the current experimental condition of $900^{\circ} \mathrm{C}$, the solubility of $\mathrm{GaN}$ in $\mathrm{Ga}$ is very small and equal to the solubility of $\mathrm{N}$ in molten Ga, which is $1.5 \times 10^{-5}(\mathrm{at} \%)$ at $900^{\circ} \mathrm{C}$ [Grossner, M. T., 1999]. Therefore the interfacial energy between $\mathrm{Ga}$ and $\mathrm{GaN}$ is calculated to be $0.25 \mathrm{~J} / \mathrm{m}^{2}$ using the above model. The surface free energy of $\mathrm{GaN}$ is taken as $2 \mathrm{~J} / \mathrm{m}^{2}$ [Northrup, \& Neugebauer, 1996]. The spreading coefficient $\mathrm{S}$ according to equation (5.2) is $1.05 \mathrm{~J} / \mathrm{m}^{2}$, which is bigger than 0 , meaning the self-spreading of nitrogenated Ga over GaN under experimental conditions. 


\subsubsection{Flow of Molten Gallium}

To study the spreading phenomena of gallium over the substrate during the nitridation, we conducted a series of experiments to find out the wettability of gallium over different substrates under different conditions.

First a gallium droplet was put onto an amorphous quartz substrate at room temperature in air, it stays as what it is and does not spread. But a nice gallium film could be made out of the droplet by smearing the droplet using another piece of glass. Then the substrate with gallium film was put into the vacuum chamber and the film stayed as it was at room temperature in vacuum. The film gradually broke up with the increase of the substrate temperature and agglomerated into several droplets at above $600{ }^{\circ} \mathrm{C}$ in vacuum. The droplets stayed as droplets after the temperature of the substrate cool down to room temperature in vacuum. The same phenomena happen under $\mathrm{N}_{2}$ and $\mathrm{H}_{2}$ atmosphere. All these results indicate that Ga itself does not wet quartz substrate. The reasoning of forming gallium film in air by smearing in air is suggested to be due to the native gallium oxide on gallium surface. The affinity between gallium oxide and oxide substrates promotes the formation of gallium film. This is also supported by the fact that a gallium film could not form on other non-oxide substrates such as polycrystalline AlN, pyrolytic $\mathrm{BN}$, and carbon through smearing the droplets.

Another experiment was done by putting a gallium droplet onto quartz substrate and exposed to nitrogen plasma to investigate the spreading phenomena. The optical image in Fig. 5.2(a) shows the spreading of the gallium over the substrate after nitridation experiment. A layer of gallium nitride was found directly on quartz substrate in the region outside of the original Ga droplet from the cross sectional image shown in Fig. 
5.2(b). On top of this gallium nitride layer, there was a layer of gallium with decreasing thickness away from the original gallium droplet. These phenomena indicate that a layer of gallium nitride first formed around the original gallium droplet through reaction between evaporated $\mathrm{Ga}$ and atomic nitrogen and then the gallium oozed out from the crust on top of Ga droplet through some cracks and spread over the gallium nitride. The crack could have been generated by the bubbling of nitrogen from the decomposition of GaN which has been observed during the experiments. The experiment with addition of $2 \mathrm{sccm} \mathrm{H}_{2}$ after the formation of GaN crust on top of gallium results in faster spreading of the Ga due to etching effect of atomic hydrogen on the GaN. The crust was broken due to the release of $\mathrm{H}_{2} / \mathrm{N}_{2}$ and gallium spread onto $\mathrm{GaN}$.
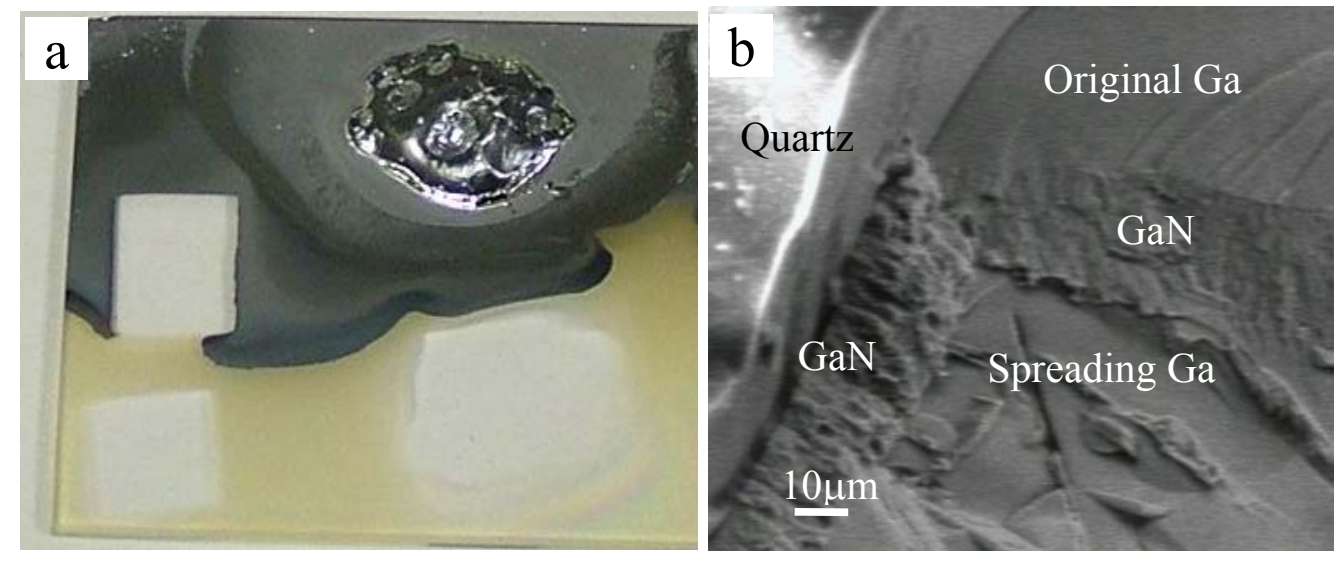

Figure 5.2 Spreading of Ga over substrates: (a) Optical photograph illustrating the spreading phenomenon of gallium over quartz substrates (b) Cross sectional SEM micrograph of the region near the original $\mathrm{Ga}$ shows the $\mathrm{GaN}$ formation and subsequent Ga film spreading onto it.

The nitridation experiment of Ga droplets with $\mathrm{NH} 3$ clearly shows the dynamic processes of GaN formation from Ga droplets. (Fig. 5.3) SEM images of different stage of nitridation processes indicate the wetting of Ga onto GaN. In the initial stages (Fig.5.3 (a)), Ga was a droplet and then it started to spread onto GaN around the droplets. With 
more GaN formation, the Ga droplet flattened further and reached equilibrium (Fig. 5.3

(b)). Ga droplets started to shrink their sizes with more Ga was converted to $\mathrm{GaN}$ and finally all the Ga was consumed and only GaN left (Fig. 5.3 (c)\&(d)).
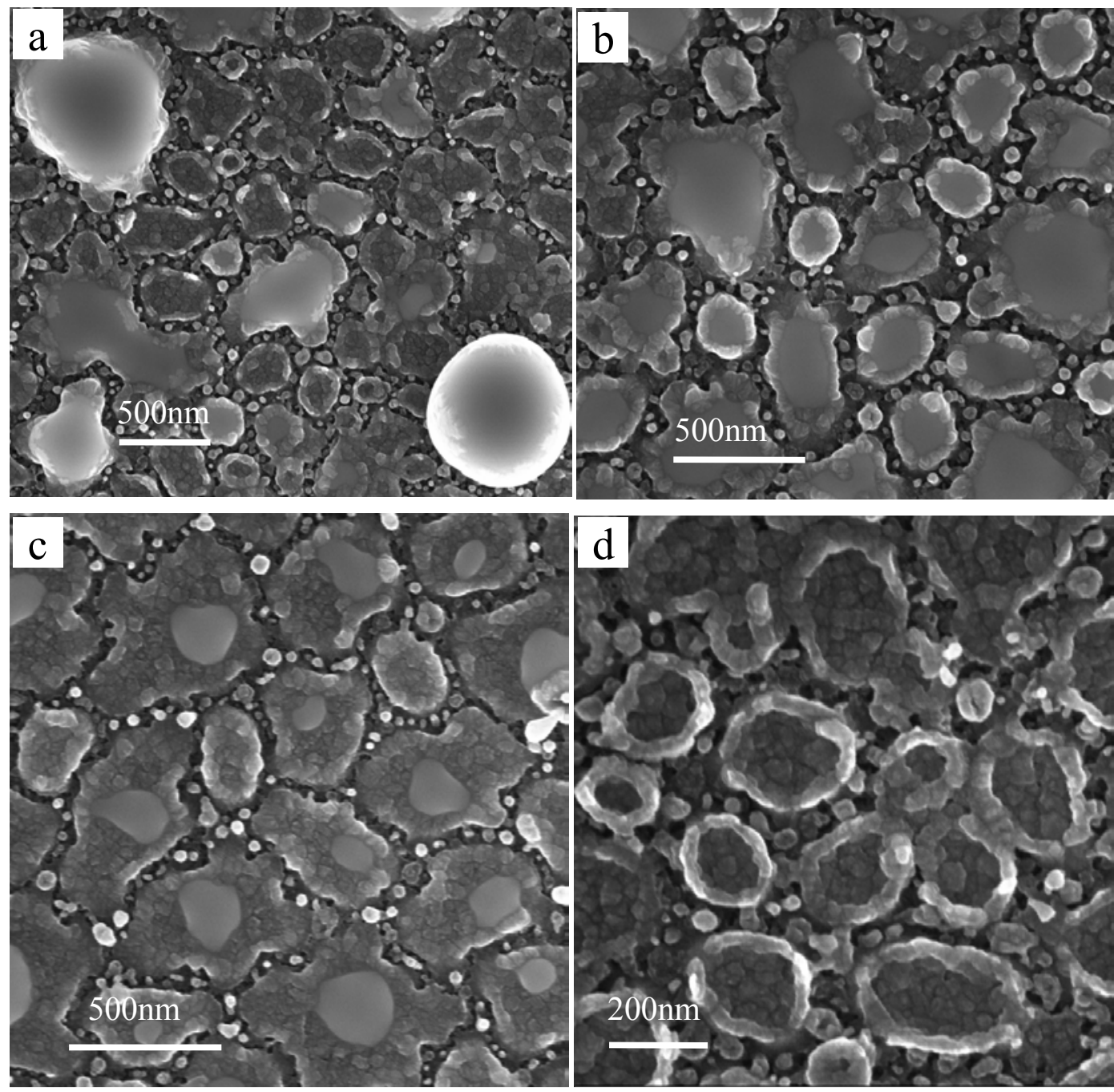

Figure 5.3 SEM photographs illustrating wetting of $\mathrm{Ga}$ on $\mathrm{GaN}$ during nitridation of $\mathrm{Ga}$ droplets using $\mathrm{NH}_{3}$.

\subsubsection{Mechanistic Studies of GaN Crystal Growth}

The understanding of the habits of GaN single crystals and their evolution is very important to understand the growth mechanism for achieving large area single crystals. 
Also, having the same morphology for GaN crystals is essential in controlling the orientation and joining of these crystals.

\subsubsection{Morphologies of GaN crystals}

\subsection{Wulff Theory of Crystal Morphology Applied to GaN Crystal Growth}

Wulff theorem, first enunciated in 1901 [Herring, C., 1951], states that a crystal grows and attains a shape with minimum total surface free energy under equilibrium conditions. It is also known that the growth rate of a surface increases with the increasing of attachment energy [Hartman, P. \& Bennema, P., 1980] which means planes with high surface energy grow faster. This is consistent with Wulff theorem since the faster growing planes eventually would disappear from the crystal and only those planes with low surface energy will be left. Hartman calculated the attachment energy of hcp crystals and found that the growth rate of some major planes has following relationship [Hartman, P. \& Bennema, P., 1980]: $R\{11 \overline{2} 0\}>R\{10 \overline{1} 1\}>R\{10 \overline{1} 0\}=R\{0001\}$. This explains why hexagonal platelets and prisms formed by $\{1010\}$ planes and $\{0001\}$ planes are mostly found in GaN crystal growth. However, if the actual growth conditions are far from the equilibrium conditions, kinetic growth may dominate and determine the final shapes of grown crystals.

\subsection{Unusual Morphologies of GaN Crystals}

Generally, plate-like habit and needle-like pyramidal prism are the most often observed morphologies of GaN single crystals. It was found that low supersaturation favors plate-like crystals growth, while high supersaturation leads to needle-like habits in high pressure solution growth [Porowski, S. \& Grzegory, I., 1997; Prywer, J. \& Krukowski, S., 1998]. Some researchers also found that high concentration of Ga 
promotes lateral growth to form plate-like crystals, while N-rich condition dominates needle-like crystal growth [Balka, C. M., et al., 2000; Callahan, M., et al., 1999; Shin, H., Z. Sitar, et al., 2002].

After the gallium was exposed to $\mathrm{N}_{2}$ plasma under high temperature, the atomic nitrogen dissolved into the gallium and the resulting melts wet the substrates very well. Spontaneous nucleation and growth formed (0002) oriented GaN layers on top of gallium. Most of the GaN crystals were micron size and plate-like (Fig. 5.4 (a)). But some standing, hollow and irregular platelets showed up in some regions when an alumina-coated tungsten coil heater was used (Fig. 5.4 (b)). A close look of these crystals was shown in Fig. 5.5 (a) \& (b). There were several narrow struts between the top layer and the bottom layer of the platelets. Some of these struts were at the edges of the platelets. A layer-by-layer growth mode was also clearly seen on the surfaces of both layers of the platelets.
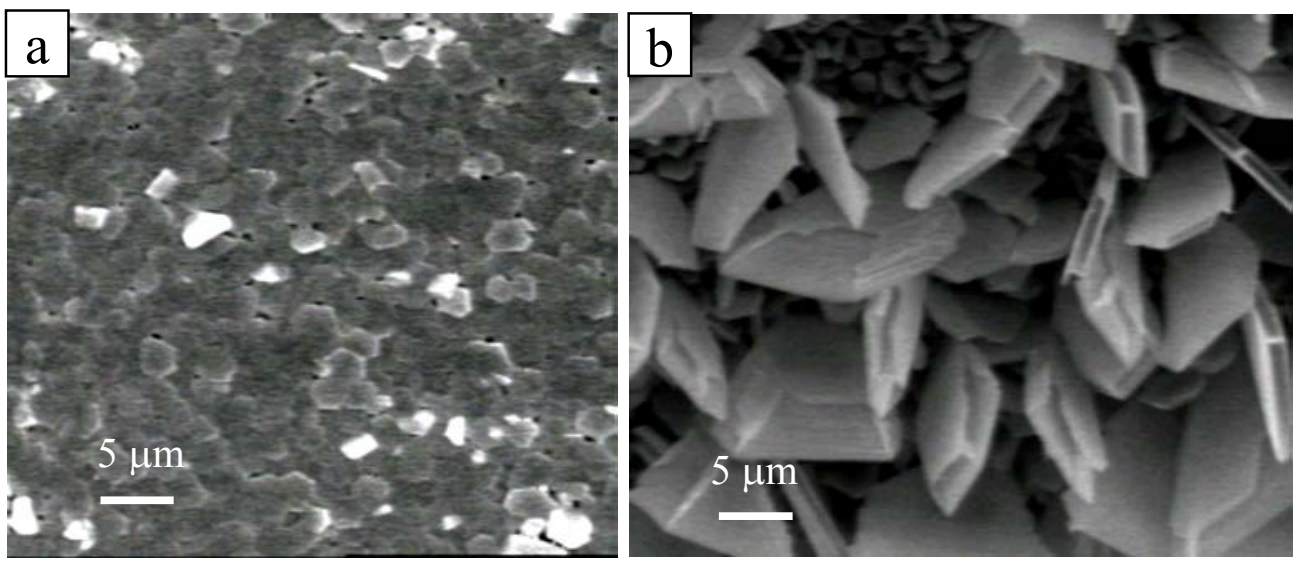

Figure 5.4 (a) GaN platelets-shaped crystals (b) Standing hollow platelets crystals. 

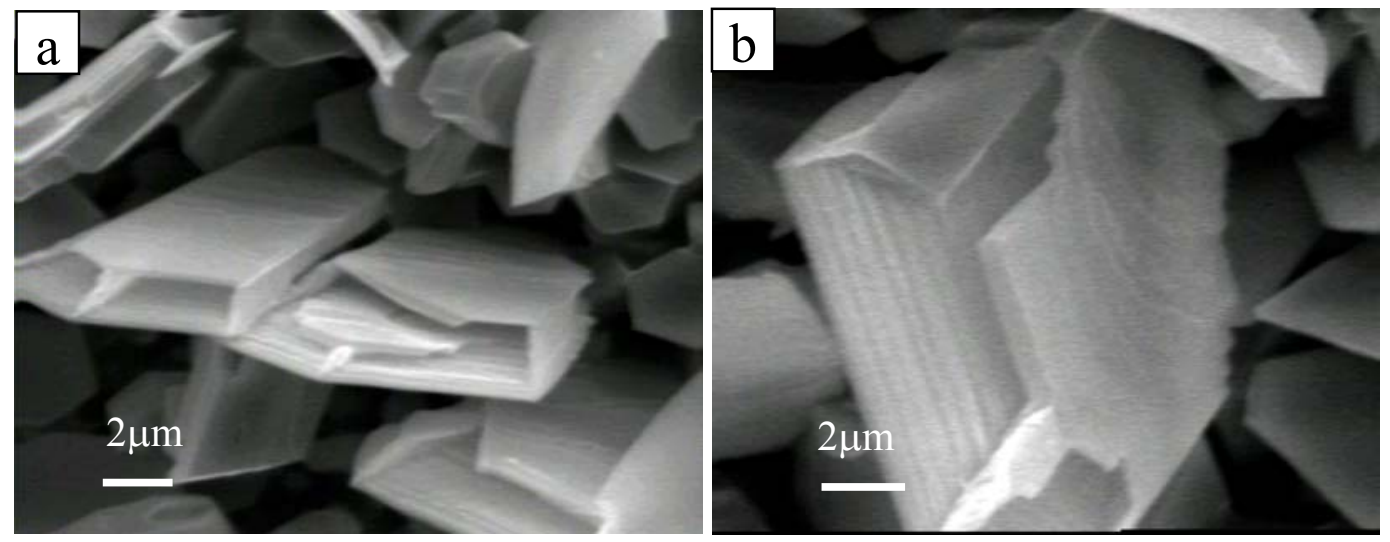

Figure 5.5 Standing GaN hollow platelets with struts between two layers indicating growth instability due to convection of gallium.

It is well known that convection in the melts influences crystal growth kinetics, compositional homogeneity, nucleation and morphological stability [Wilcox, W. R., 1983]. Preliminary analysis showed that convection does exist due to the non-uniform heating of the W-coil heater [Chandrasekaran, H., 2002]. The solute concentration on the crystal surface varies even without convection. With convection, especially strong Marangoni convection present in our experiment [Chandrasekaran, H., 2002], the platelet-shaped crystals were forced to stand in the solution firstly. Stable layer-by-layer growth can only occur if the step formation is slower than step propagation. But step generation usually happens where the supersaturation is the highest. In our experiment, the solute concentration was highest at the edge of the crystals where the flow first meets the crystals and decreases downstream due to the convection. Also the solute concentration at the two side-edges that were almost parallel to the flow was higher than that at the center since there was more bulk solution around the edges. Therefore steps formed primarily at the edge of crystals. The convection was strong enough to affect the kinetics and step propagation was slower than step generation. The formation of top layer 
was possible due to the stable growth mode when the convection was not strong. Finally these hollow platelets with narrow struts between two layers formed.

Some crystals with a depression at the center of top face were shown in Fig. 5.6. The depressions exhibit varying depth and irregular shapes for different crystals. The inset of Fig 5.6(a) shows a closed look of one such shallow depression. A layer-by-layer growth mode was clearly seen. The layers were trying to propagate to the center from the edge and the top layers stopped growing further away from the center.

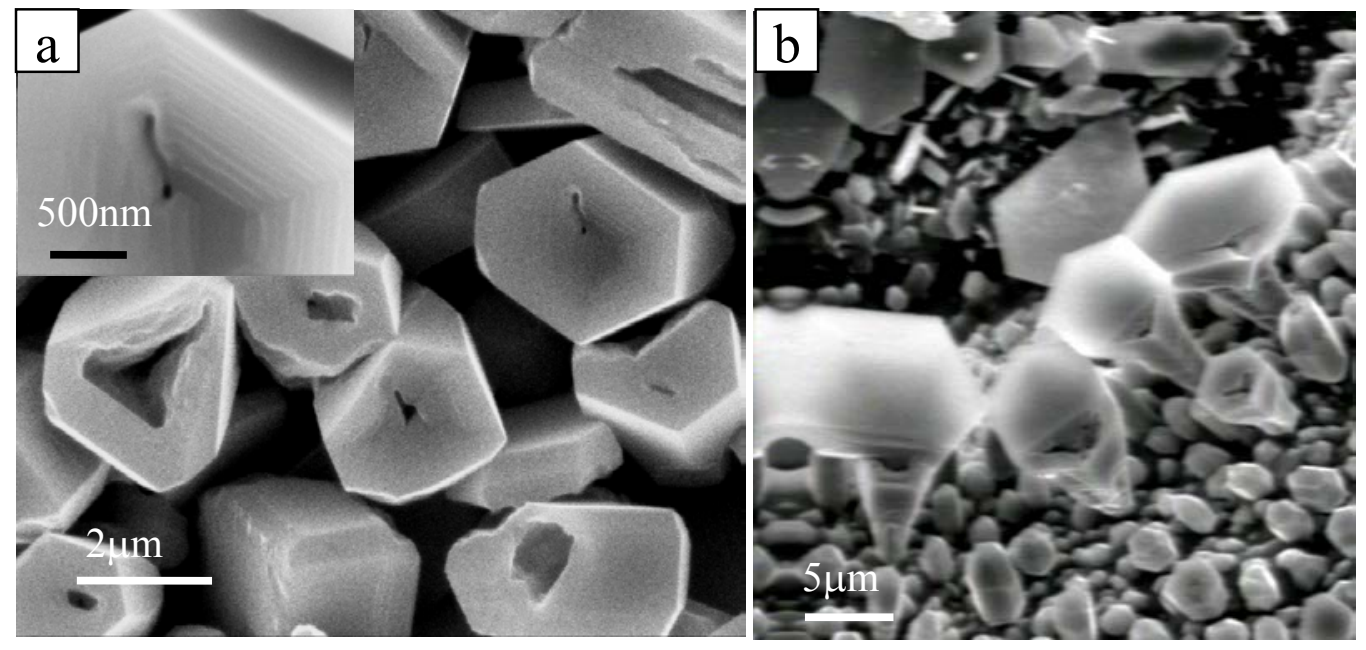

Figure 5.6 GaN crystals with variable depressions on top surfaces.

It is found that Ga wets GaN very well under high temperature [Logan, R.A. \& Thurmond, C.D., 1972] and our experiments had also showed this phenomenon. When the GaN crystals grew to certain size and they were not fully immersed into the molten gallium, Ga crept up the crystals due to spontaneous wetting and formed a thin layer gallium on the surfaces of the crystals. Simultaneously, the liquid phase epitaxy could occur through the gallium film with dissociation of nitrogen atoms from the gas phase. As the top surfaces of these crystals were further above the surface of the molten Ga due to the growth of crystals or the consumption of $\mathrm{Ga}$, it became more difficult for the gallium to creep up and spread further towards the center of top faces. Gradually, an 
inverted cone-shaped depression developed. This phenomenon is further supported by some crystals whose depressions are further away from one side which was close to a gallium pool. (See Fig. 5.6(b))

Some crystals exhibited six-arm star-shaped morphology in the experiment with 10:1 of $\mathrm{N}_{2}: \mathrm{H}_{2}$ (Fig. 5.7). Most of the star-shaped crystals have a pyramidal top (Fig. 5.7(a)), but some of them have a flat but rough top surface (Fig. 5.7(b)), or even with some pits (Fig. 5.7(c)). The side view (Fig. 5.7(d-f)) shows that the star-shaped crystals with pyramid top have a star-shaped column at the bottom. The angles of six-arms measured from the SEM images varied from around $70^{\circ}$ to $110^{\circ}$, but most of them are around $90^{\circ}$. This measurement is not accurate since crystals are three-dimensional but SEM images are only two-dimensional. However it seems that these stars-shaped crystals do not have perfect six-fold symmetry.
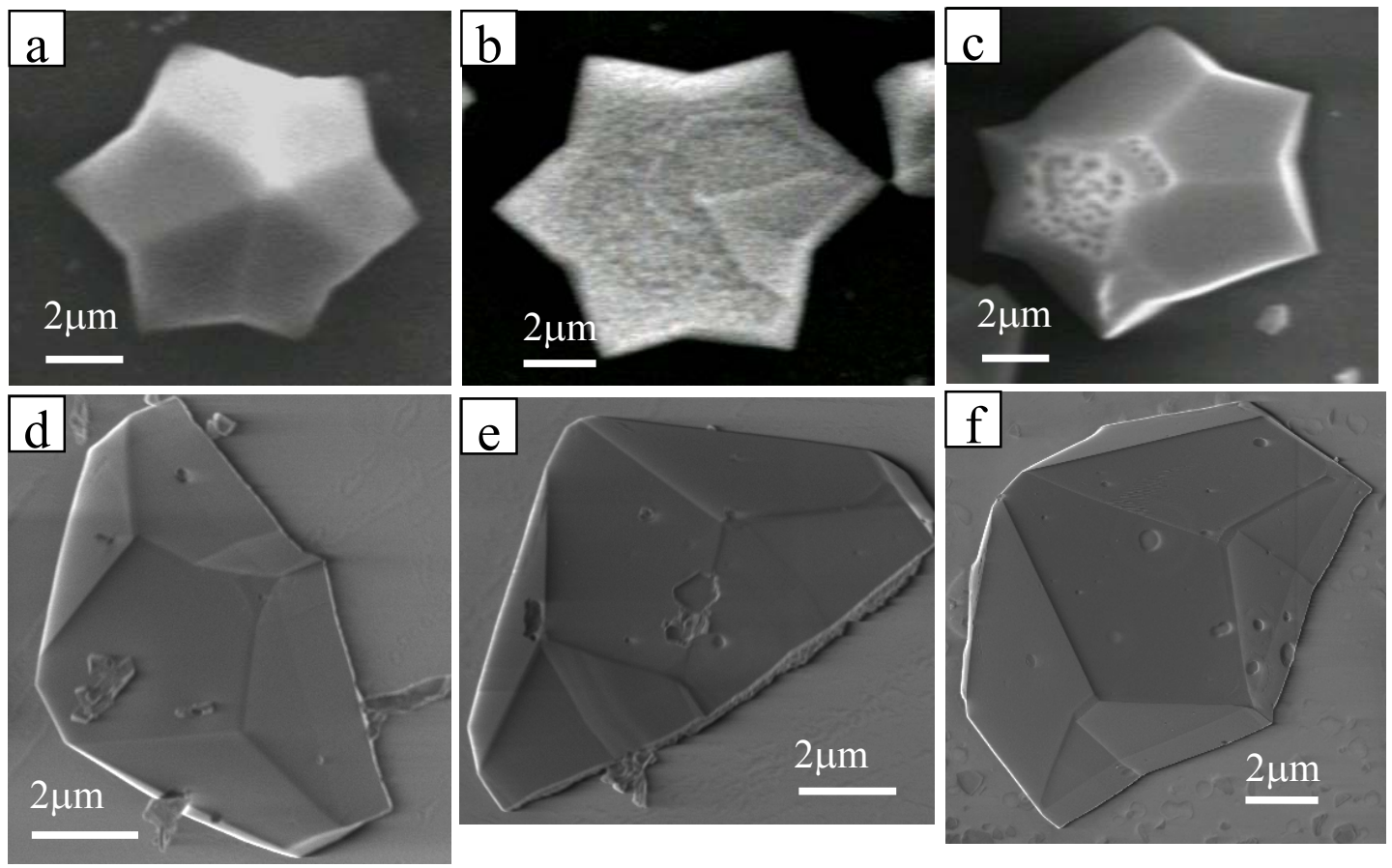

Figure 5.7 Various star-shaped GaN crystals; (a-c) Bird-view images (d-f) Side-view images. 
Geometrically, there are many kinds of stars one could draw from a hexagon. There are two basic types of equal lateral star-shaped morphologies depending where the six arms originate: edge (Fig. 5.8 (a)) or corner (Fig. 5.8 (d)). Fig. 5.8 shows four different six-fold symmetrical stars bounded by the same group low index planes. Among them, (b) and (e) with an angle of $60^{\circ}$ for the arms are bounded by $\{1120\}$ planes and $\{20 \overline{2} 0\}$ respectively, (c) and (f) are bounded by $\{2130\}$ planes but they have different arm angles of $82^{\circ}$ and $98^{\circ}$, respectively. Considering the side faces of the pyramid top, they could be $\{11 \overline{2} n\}$ for the star shown in Fig. 5.8 (a) and $\{10 \overline{1} n\}(\mathrm{n}=1,2)$ for the one shown in Fig. 5.8 (d), respectively. Comparing with the configuration of those star-shaped crystals, it was found that they are more like the star shown in Fig. 5.8(c). They should have side pyramidal faces of $\{10 \overline{1} n\}$ since $\{10 \overline{1} n\}$ planes have been found in GaN crystals grown with different techniques, but $\{11 \overline{2} n\}$ has only been seen in lateral epitaxial overgrown (LEO) GaN under certain condition [Hiramatsu, K., et al., 2000]. This is further supported by the angle between the side pyramidal face and base plane shown in Fig. 5.9. They are around $42^{\circ}$ to $46^{\circ}$, close to the $43^{\circ}$, which is the angle between $\{1012\}$ and $\{0001\}$ planes. 


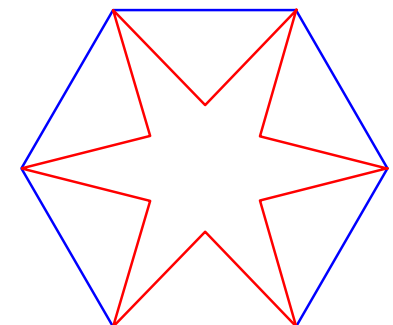

(a)

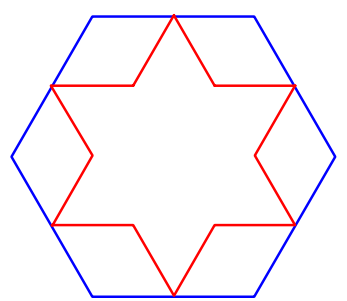

(d)

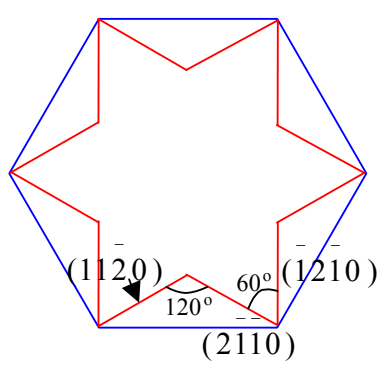

(b)

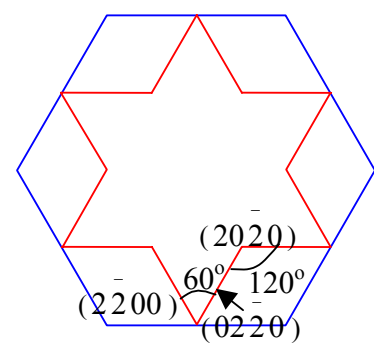

(e)

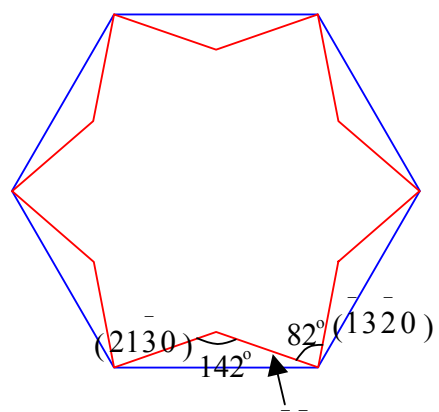

(c)

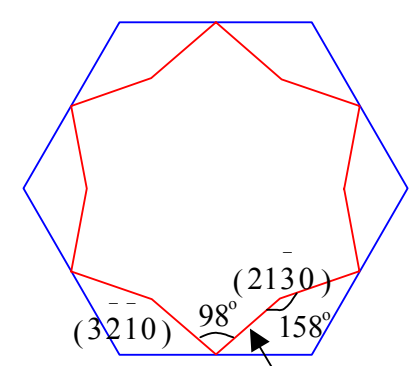

(f)

(1230)

Figure 5.8 The star morphologies with 6-fold symmetry from a hexagon: (a)\&(d) The two basic kinds of stars with 6-fold symmetry from a hexagon; (b, c, e, f) Four different kinds of six-fold symmetrical stars bound by low index planes $(\leq 3)$.
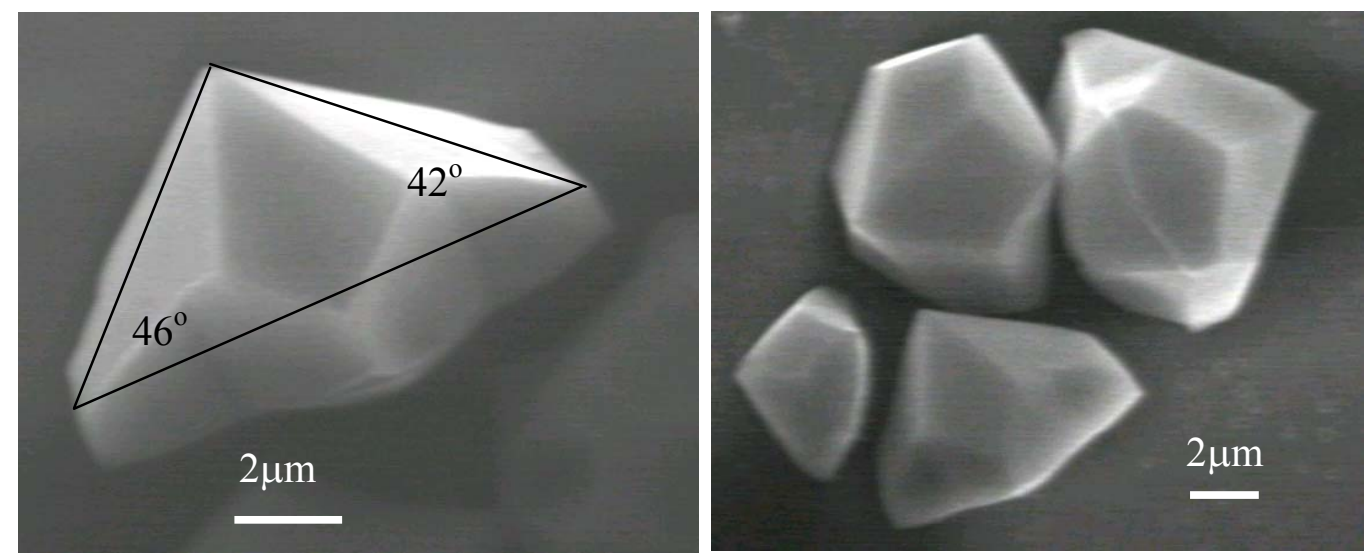

Figure 5.9 Side views of the star-shaped GaN crystals.

It has been widely known that hexagonal platelets and prisms bounded by $\{10 \overline{10}\}$ planes and $\{0001\}$ planes are the mostly often seen morphologies for GaN crystals. The 
facets of star-shaped crystals shown in Fig. 5.8 cannot be explained using the above theory. The observed facets with high index planes may have possibly occurred due to the kinetic faceting under the presence of $\mathrm{H}_{2}$. $\mathrm{GaN}$ is believed to decompose under high temperature [Shin, H., et al., 2002.] and the adsorption of active hydrogen in the plasma on the surface of $\mathrm{GaN}$ crystals promotes the decomposition of $\mathrm{GaN}$ and might stabilize those surfaces.

\subsubsection{Sequential Growth of Self-oriented GaN Film}

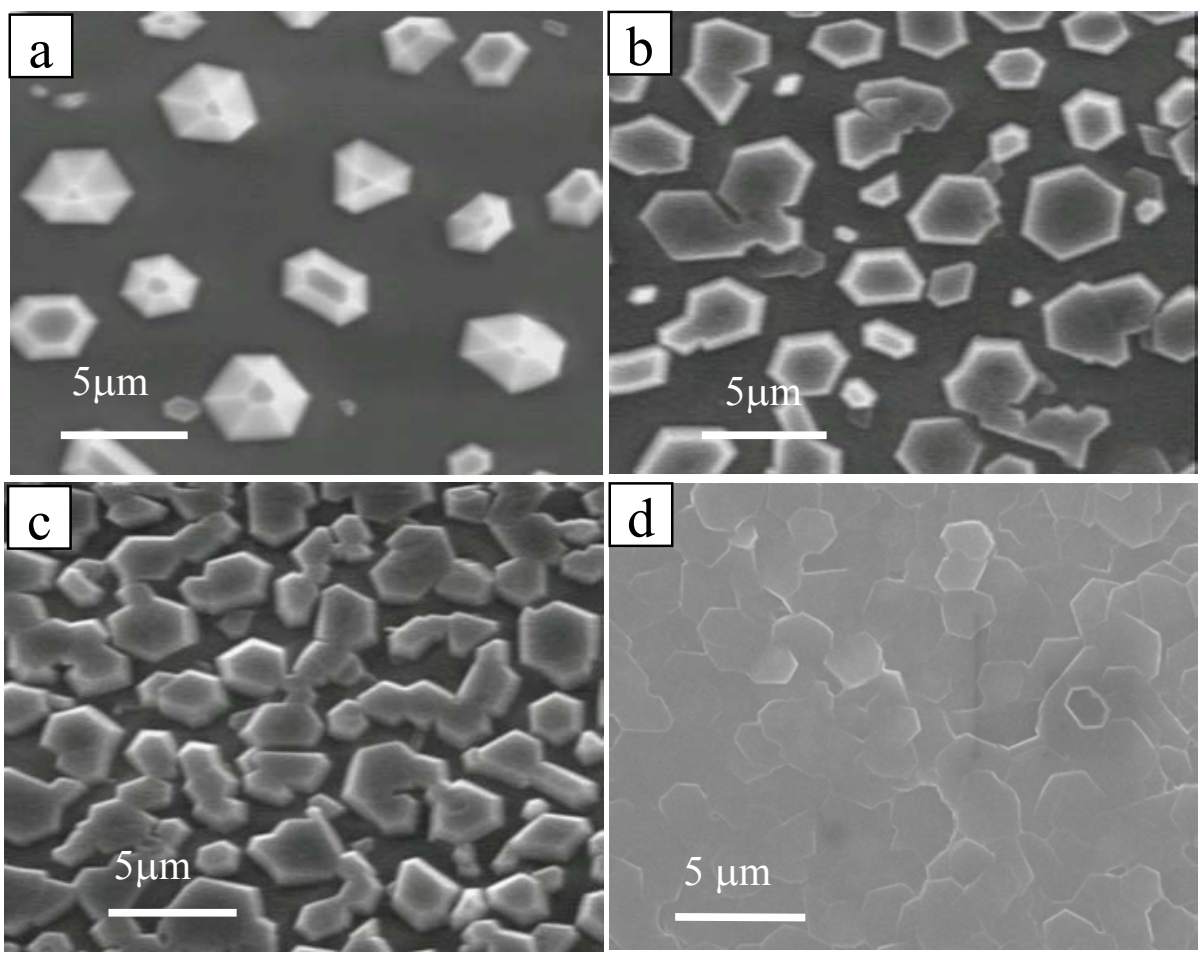

Figure 5.10 (a-d) Plan view SEM images of sequential growth of oriented $\mathrm{GaN}$ on top of gallium.

SEM images of the GaN films at different time scales during nitridation experiments in Fig. 5.10 clearly show various stages involved with coalescing of the GaN platelets growing on top of molten gallium. 
A layer of molten gallium can be seen from the cross sectional SEM micrographs in Fig. 5.11. GaN platelets grew with both basal attachment and top growth through liquid phase epitaxy before they fully coalesced to form a film. This process is justified since the atomic nitrogen could dissolve into $\mathrm{Ga}$ and diffuse to the bottom surface of $\mathrm{GaN}$ platelets. Also a layer of molten Ga could flow over the GaN platelets due to wetting and develop liquid phase epitaxy. Cross sectional TEM analysis described in the following section is also consistent with the above observations. The basal growth stopped after the coalescence since atomic nitrogen could not diffuse through the GaN layer. Further growth might still occur through liquid phase epitaxy in a layer of Ga on top of $\mathrm{GaN}$ films. The Ga layer on top of GaN films could come from the Ga flow through cracks in the GaN films.

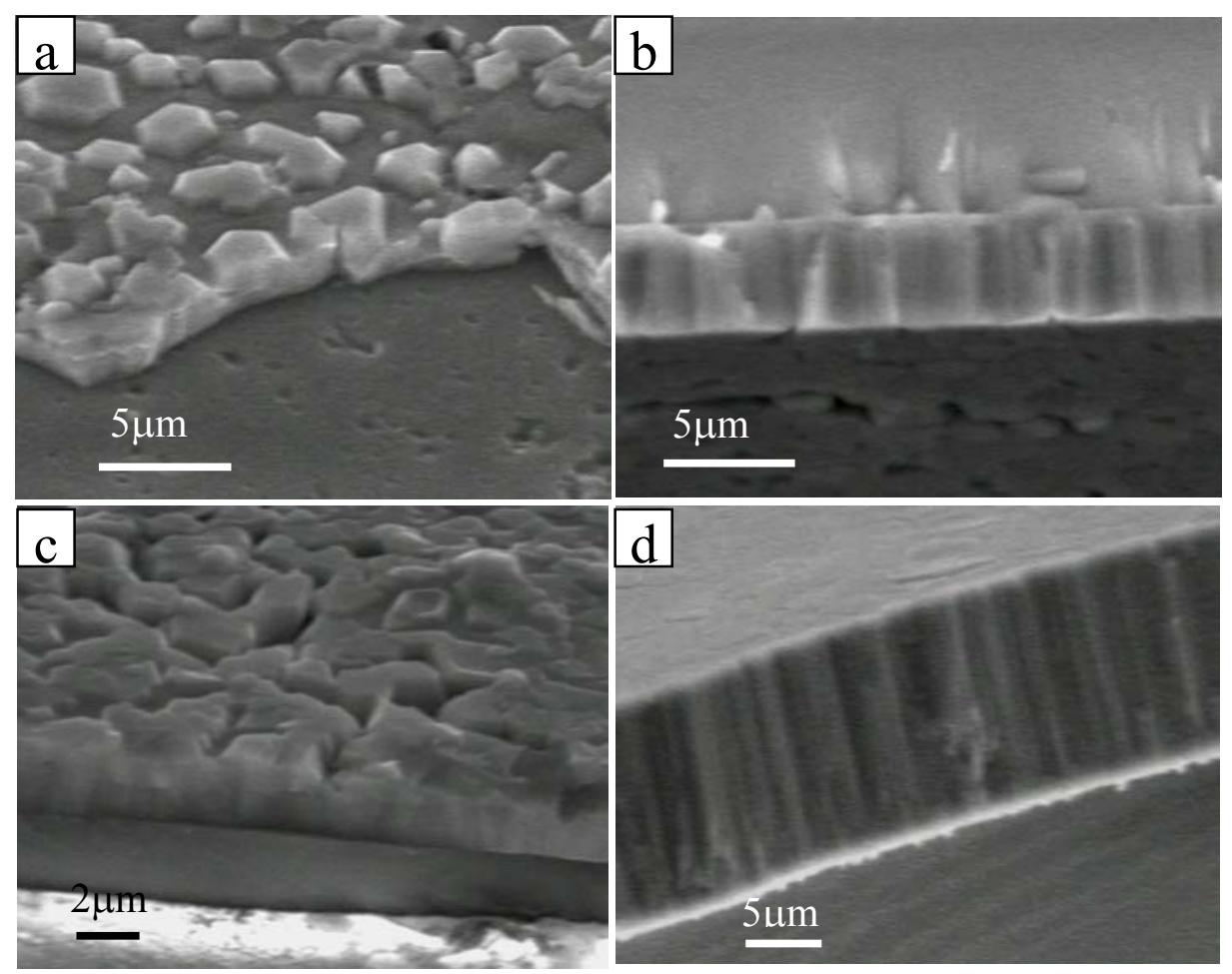

Figure 5.11 (a-d) Cross sectional SEM micrographs of sequential growth of oriented GaN on top of gallium. 


\subsection{Large Area Self-Oriented GaN Film Growth on Molten Ga}

\subsubsection{Oriented GaN on Molten Ga}

The spreading of gallium melts was found to be very critical in forming the smooth textured films as shown in Fig. 5.12. The X-ray diffraction spectrum of the GaN film formed on top of Ga from the experiment when Ga spread during nitridation showed only reflections of (0002) and (0004) planes of wurtzite GaN (Fig. 5.13(a)), indicating good c-plane orientation. The XRD spectrum of GaN crust on top of Ga melt when Ga did not exhibit spreading is shown in Fig. 5.13(b). This spectrum shows all the characteristic XRD peaks of wurtzite $\mathrm{GaN}$ indicating random orientation. The crosssectional SEM image of sample that exhibited a smooth textured GaN clearly indicates the presence of a layer of $\mathrm{Ga}$ in between the substrate and the GaN film as shown in Fig. 5.14(a). A representative image shown in Fig. 5.14(b) is for GaN films without the presence of any molten Ga buffer layer on amorphous quartz substrate. The difference between Figs. 5.14 (a) \& (b) is that the crystals grown on molten Ga buffer layer selfassembled to form an initial smooth textured film. 


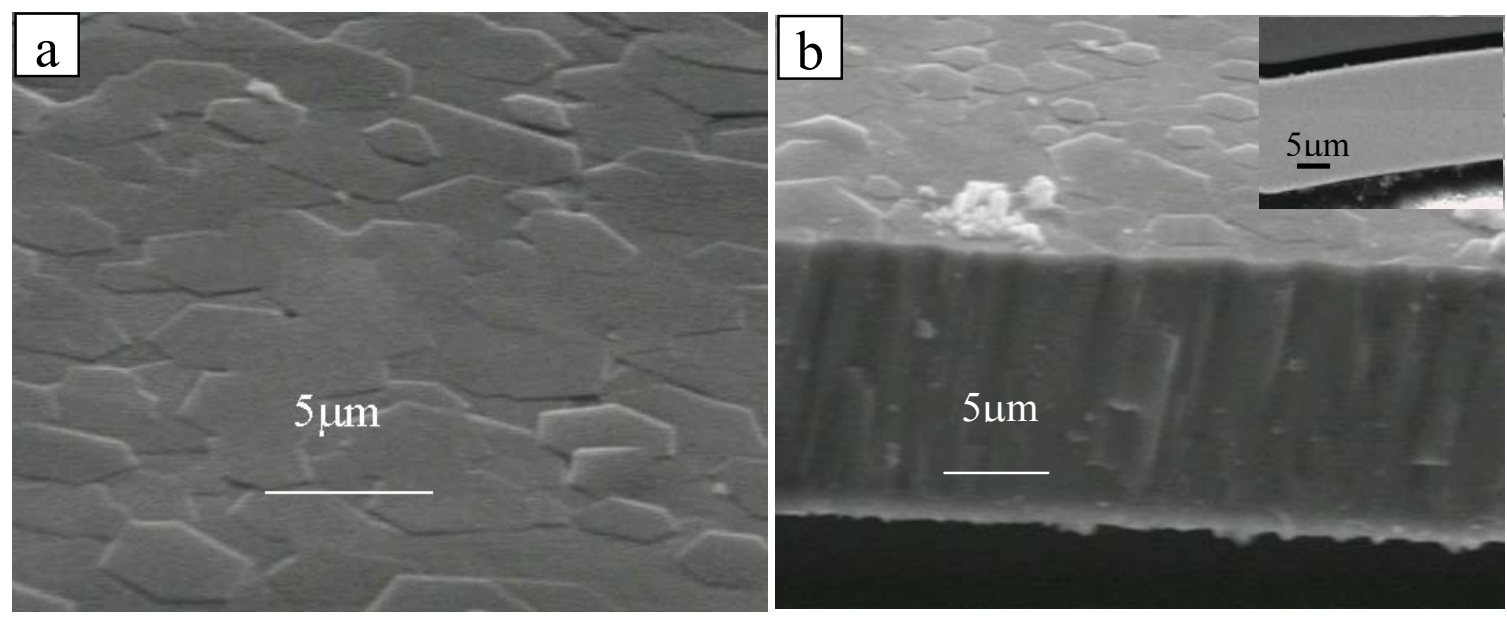

Figure 5.12 Representative SEM images of free-standing, c-plane oriented GaN films after Ga was dissolved away: (a) plan view and (b) cross section view. Inset in (b) shows SEM image of a cross sectional TEM sample after polishing.
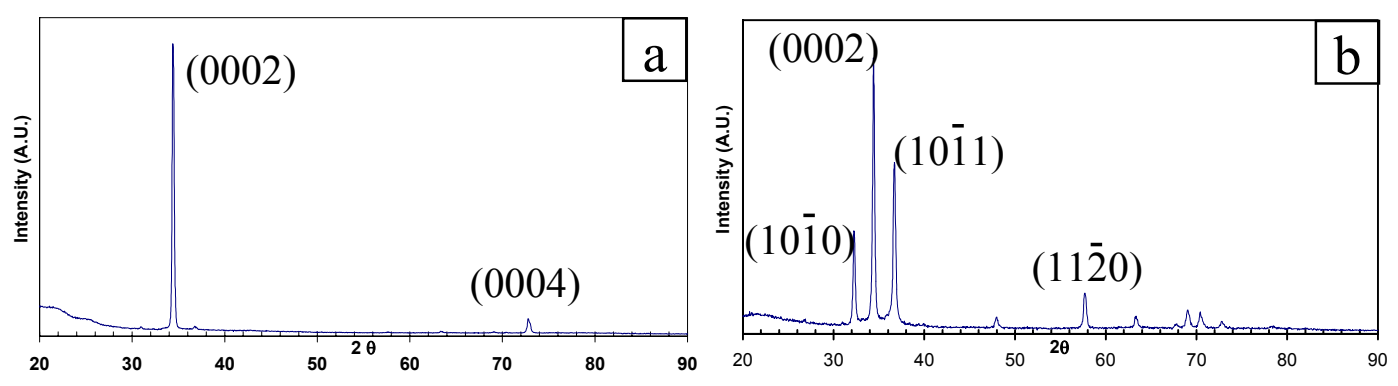

Figure 5.13 XRD spectra of GaN films obtained under two different wetting conditions of molten Ga. (a) Nitridation experiment when Ga melt spreads and (b) GaN crust on Ga when Ga does not spread. 

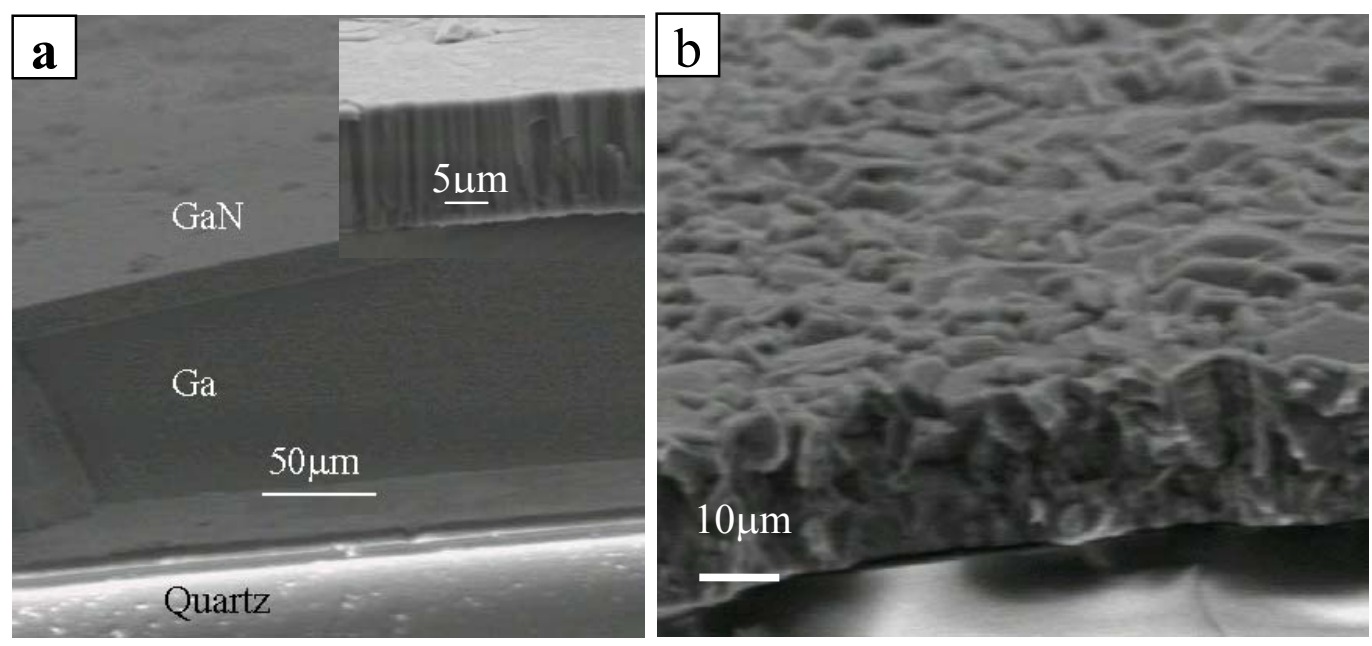

Figure 5.14 The SEM images of two different GaN films: (a) GaN grown directly on top of $\mathrm{Ga}$ and (b) GaN grown directly on the amorphous Quartz substrate without the Ga buffer layer.

\subsubsection{Polarity of the GaN Film on Ga}

It is widely known that wurtzite $\mathrm{GaN}$ is a polar material in (0001) orientation, which has Ga-terminated surface (0001) and N-terminated surface (000-1). The surface properties of GaN as well as device performance have been shown to depend upon different polarities [Bykhovski, A., Gelmont, B. \& Shur, M., 1993; Asbeck, P. M., et al., 1997; Shur, M. S., Bykhovski, A. D., Gaska, R., 1999]. Therefore it is very important to know and control the polarities of $\mathrm{GaN}$ since most $\mathrm{GaN}$ films are epitaxially grown with (0001) orientation. Several techniques have been developed to determine the polarities. These include CBED technique [Ponce, F.A., et al., 1996], Auger spectroscopy [Khan, M. A., et al., 1993], Rutherford backscattering ion channeling technique [Daudin, B., Rouviere, J. L., Arlery, M., 1996.], RHEED [Held, R., et al., 1999; Molnar, R.J., Singh, R., Moustakas, T.D., 1995] and chemical etching [Stocker, D. A, et al., 1998; Weyher, J.L., et al., 2000; Hong, S.K., et al., 2000]. 
Chemical wet etching has been used as a simple technique to study the polarities of semiconductors as well as defects in the semiconductor materials [Kern, W., 1976; Tijburg, R., 1976]. For polar GaN material, an alkaline solution (KOH or $\mathrm{NaOH})$ has been found to have different reactivities on the surfaces with different polarities. It is found that the solution will selectively attack defective sites on the Ga-terminated surface and forms pits, while etches $\mathrm{N}$-terminated surface quickly and form hexagonal hillocks on the surface [Weyher, J.L., et al. 2003]. The polarity of the oriented GaN films on Ga was determined using chemical etching in $6 \mathrm{M} \mathrm{KOH}$ for 15 minutes at $60{ }^{\circ} \mathrm{C}$. SEM images in Fig. 5.15 show that there are pits developed especially at the grain boundaries on the top surface. This indicates that it is Ga-terminated and there are defects at the boundaries due to non-perfect joining. The presence of lots of hexagonal hillocks on the bottom surfaces after etching indicates $\mathrm{N}$-terminated surface. The polarities of the GaN crystals grown in this work are the same as those grown from molten Ga solution under high pressure and high temperature [Hellman, E. S., 1998]. 

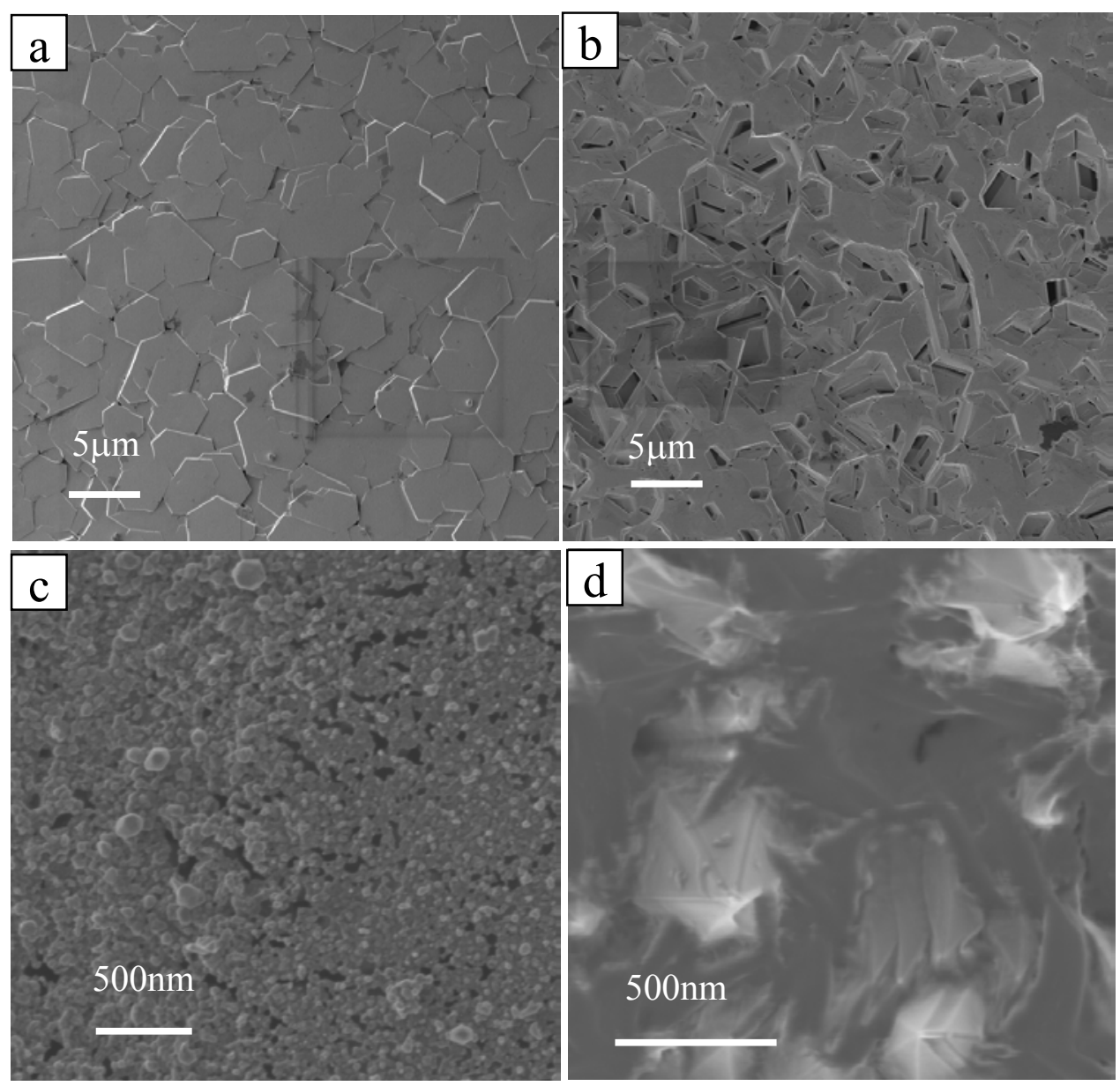

Figure 5.15 SEM images of GaN film with $\mathrm{KOH}$ etching for polarity determination. (a)

Top surface before etching; (b) Top surface after etching; (c) Bottom surface before etching; (d) Bottom surface after etching.

\subsubsection{Stresses and Native Carrier Concentration of GaN Grown on Ga}

Wurtzite GaN, which belongs to space group P6 $6_{3}$ mc, has 6 optical modes, $\mathrm{A}_{1}, \mathrm{E}_{1}$, $2 \mathrm{E}_{2}, 2 \mathrm{~B}_{1}$. Among these optical modes, $\mathrm{B}_{1}$ modes are silent, non-polar $\mathrm{E}_{2}$ modes are Raman active only, both polar modes $\mathrm{A}_{1}$ and $\mathrm{E}_{1}$ are both Raman and infrared active and they split into longitudinal (LO) and transverse (TO) components due to the anisotropy of the macroscopic electric field resulting from the strong ionic bonds in GaN. For cubic GaN, if backscattering geometry is employed with no polarization detection, only LO 
phonon is observed from (100) plane, TO phonon is observed from (110) plane. Both phonons show up from (111) plane. The allowed Raman modes and their frequencies for wurtzite GaN are summarized in Table 5.1.

Table 5.1 Raman modes and their frequencies for wurtzite GaN [Harima, H., 2002].

\begin{tabular}{|l|l|l|}
\hline Modes & Configurations for the allowed modes $*$ & Phonon frequencies $\left(\mathrm{cm}^{-1}\right)$ \\
\hline $\mathrm{E}_{2}{ }^{1}$ & $\mathrm{z}(\mathrm{x}, \mathrm{y})-\mathrm{z} ; \mathrm{z}(\mathrm{y}, \mathrm{y})-\mathrm{z} ; \mathrm{x}(\mathrm{y}, \mathrm{y})-\mathrm{x} ; \mathrm{x}(\mathrm{y}, \mathrm{y})-\mathrm{z} ;$ & $143.5 \pm 0.5$ \\
\hline $\mathrm{A}_{1}(\mathrm{TO})$ & $\mathrm{x}(\mathrm{y}, \mathrm{y})-\mathrm{x} ; \mathrm{x}(\mathrm{z}, \mathrm{z})-\mathrm{x} ;$ & $531 \pm 0.5$ \\
\hline $\mathrm{E}_{1}(\mathrm{TO})$ & $\mathrm{x}(\mathrm{y}, \mathrm{z})-\mathrm{x} ; \mathrm{x}(\mathrm{y}, \mathrm{z})-\mathrm{y} ;$ & $558.6 \pm 0.5$ \\
\hline $\mathrm{E}_{2}{ }^{2}$ & $\mathrm{z}(\mathrm{x}, \mathrm{y})-\mathrm{z} ; \mathrm{z}(\mathrm{y}, \mathrm{y})-\mathrm{z} ; \mathrm{x}(\mathrm{y}, \mathrm{y})-\mathrm{x} ; \mathrm{x}(\mathrm{y}, \mathrm{y})-\mathrm{z} ;$ & $567.5 \pm 0.5$ \\
\hline $\mathrm{A}_{1}(\mathrm{LO})$ & $\mathrm{z}(\mathrm{x}, \mathrm{x})-\mathrm{z} ;$ & $734.2 \pm 0.5$ \\
\hline $\mathrm{E}_{1}(\mathrm{LO})$ & $\mathrm{x}(\mathrm{y}, \mathrm{z})-\mathrm{y} ;$ & $741 \pm 0.5$ \\
\hline
\end{tabular}

$*_{\mathrm{z}}$ is the direction along the c-axis; $\mathrm{x}$ and $\mathrm{y}$ directions are perpendicular to the c-axis. $\mathrm{z}(\mathrm{x}, \mathrm{x})$ - $\mathrm{z}$ scattering configuration: first $\mathrm{z}$ is the direction of incident light, second $\mathrm{z}$ is the direction of scattered light. In the parentheses, left one is the polarization direction of the incident light; right one refers to the polarization direction of scattered light.

The Raman spectra were taken using $632.8 \mathrm{~nm}$ He-Ne laser at room temperature with Renishaw InVia Raman microscope. The spectrum in normal back-scattered configuration under which only $\mathrm{E}_{2}$ and $\mathrm{A}_{1}(\mathrm{LO})$ modes are allowed for hexagonal $\mathrm{GaN}$ [Tabata, A., et al., 1996] is shown as curve a in Fig. 5.16. The spectrum shows the characteristic peaks of $E_{2}^{1}$ at $143 \mathrm{~cm}^{-1}, E_{2}^{2}$ at $567 \mathrm{~cm}^{-1}$ and $A_{1}(\mathrm{LO})$ at $739 \mathrm{~cm}^{-1}$. The curve b in Fig. 5.16 is the Raman spectrum measured with the laser beam focusing on the cross section of the GaN film where the incident beam is perpendicular to the c-axis. 
The $A_{1}(T O)$ mode at $531 \mathrm{~cm}^{-1}, E_{1}(T O)$ mode at $559 \mathrm{~cm}^{-1}$ and $E_{1}(L O)$ mode at $743 \mathrm{~cm}^{-1}$ that are absent in the back scattering configuration show up.

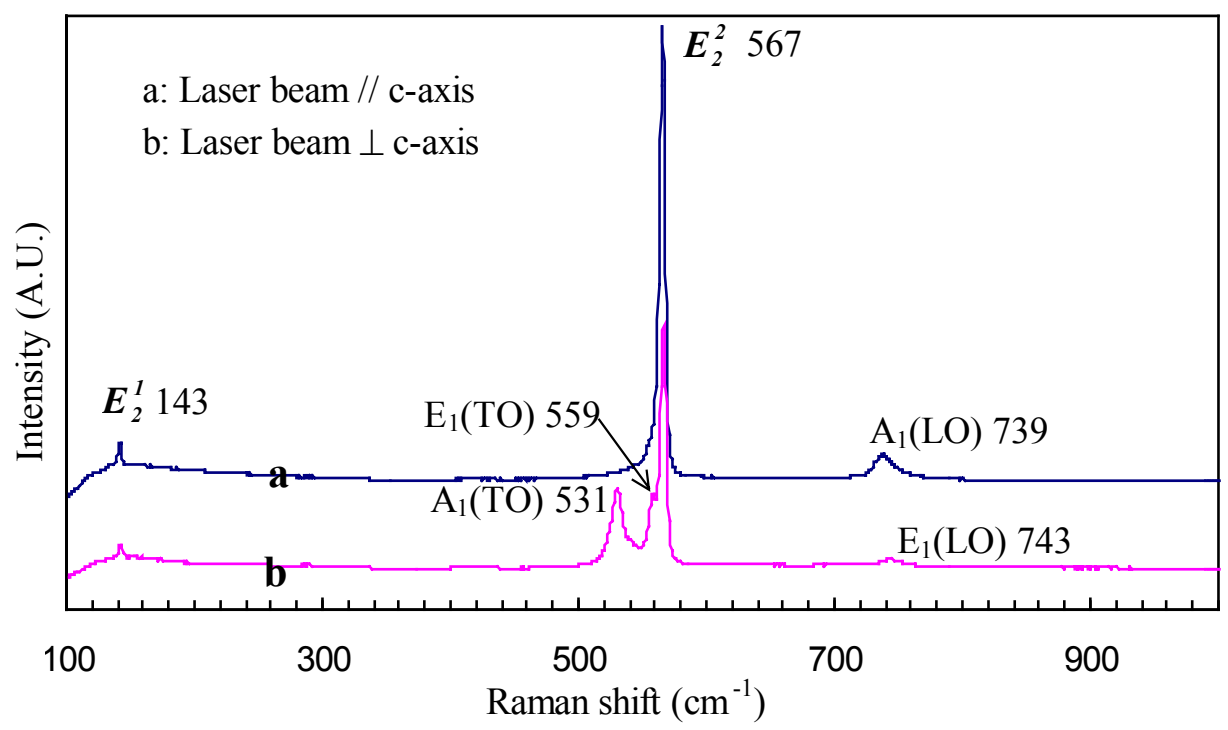

Figure 5.16 Raman spectra of oriented GaN film with laser beam parallel and perpendicular to the c-axis.

The high frequency mode, $\mathrm{E}_{2}^{2}$ originates from the vibration perpendicular to caxis. Therefore $\mathrm{E}_{2}{ }^{2}$ phonon peak is very sensitive to the biaxial strain in the c-plane and the shift of the frequency can be used to characterize the residual strain of GaN. It has been suggested that the stress-free $\mathrm{E}_{2}{ }^{2}$ phonon peak should be at $567.5 \pm 0.5 \mathrm{~cm}^{-1}$ [Harima, H., 2002]. The $\mathrm{E}_{2}{ }^{2}$ peak shifts to high frequency for $\mathrm{GaN}$ epitaxially grown on sapphire since there is compressive stress in $\mathrm{GaN}$ due to larger thermal expansion coefficient of sapphire substrates (sapphire: $7.5 \times 10^{-6} / \mathrm{K}, \mathrm{GaN}: 5.59 \times 10^{-6} / \mathrm{K}$ ). Fig. 5.17 shows the $\mathrm{E}_{2}^{2}$ phonon peaks of GaN grown on Ga, amorphous quartz and single crystalline sapphire. There is no frequency shift for GaN grown on Ga and amorphous quartz since there is no epitaxial relationship between them. But the frequency up-shifts $2 \mathrm{~cm}^{-1}$ for the $\mathrm{GaN}$ grown on sapphire substrates due to the expected compressive strain [Kisielowski, C., et al., 1996]. 
In semiconductors, the plasmons of free-electrons or free-holes are longitudinal wave-like oscillations with a frequency of $\omega_{P}$ [Colwell, P. J. \& Klein, M. V., 1972]:

$$
\omega_{P}=\sqrt{\frac{n e^{2}}{m^{*} \varepsilon_{0}}}
$$

Where $\mathrm{n}$ and $\mathrm{m}^{*}$ are the density and effective mass of the free carriers. $\varepsilon_{0}$ is the permittivity of free space. $\mathrm{e}$ is the electric charge of one electron. The damping constant of plasmons is given:

$$
\gamma_{P}=\frac{e}{\mu m^{*}}
$$

Where $\mu$ is the mobility of the carriers. The LO-phonon may couple together with plasmon oscillation when their frequencies are close and the plasmon-like component of the coupled LO-plasmon mode appears as a very broad band shifted towards high frequency for highly doped semiconductors. Under backscattered configuration, the LOphonon-plasmon coupled mode in $\mathrm{GaN}$ corresponds to the axial type of vibration that propagate along c-axis which becomes asymmetric with a longer tail at higher frequency [Perlin, P., et al., 1995]. Therefore the profile of LO-phonon-plasmon coupled modes can be used to evaluate the carriers density and mobility [Kozawa, T., et al., 1994; Harima, H., et al., 1998]. The carrier density can be approximately deduced from the frequency shift of the coupled mode (CM) [Wetzel, C., et al., 1996]:

$$
n=1.1 \times 10^{17}\left(\omega_{C M}-736\right)
$$

When the dopants concentration is very high, the LO-phonon-plasmon coupled mode is usually over-damped and could not be observed experimentally. Fig. 5.18 shows the profile of LO-phonon-plasmon coupled mode of $\mathrm{GaN}$ grown on $\mathrm{Ga}$ and amorphous 
quartz. The frequency shifted to $739 \mathrm{~cm}^{-1}$ for GaN grown on Ga indicating carrier density to be around $3 \sim 4 \times 10^{17} \mathrm{~cm}^{3}$. However, the $A_{1}$ (LO) phonon plasmon coupled mode in GaN directly grown on quartz was not observed which indicates the electron concentration is over $10^{19} \mathrm{~cm}^{-3}$ [Demangeot, F., et al., 1997]. The high electron concentration might come from the diffusion of oxygen from the quartz substrates during growth. The results clearly show that Ga buffer layer could also acts as a barrier to stop the diffusion of oxygen from the substrate.

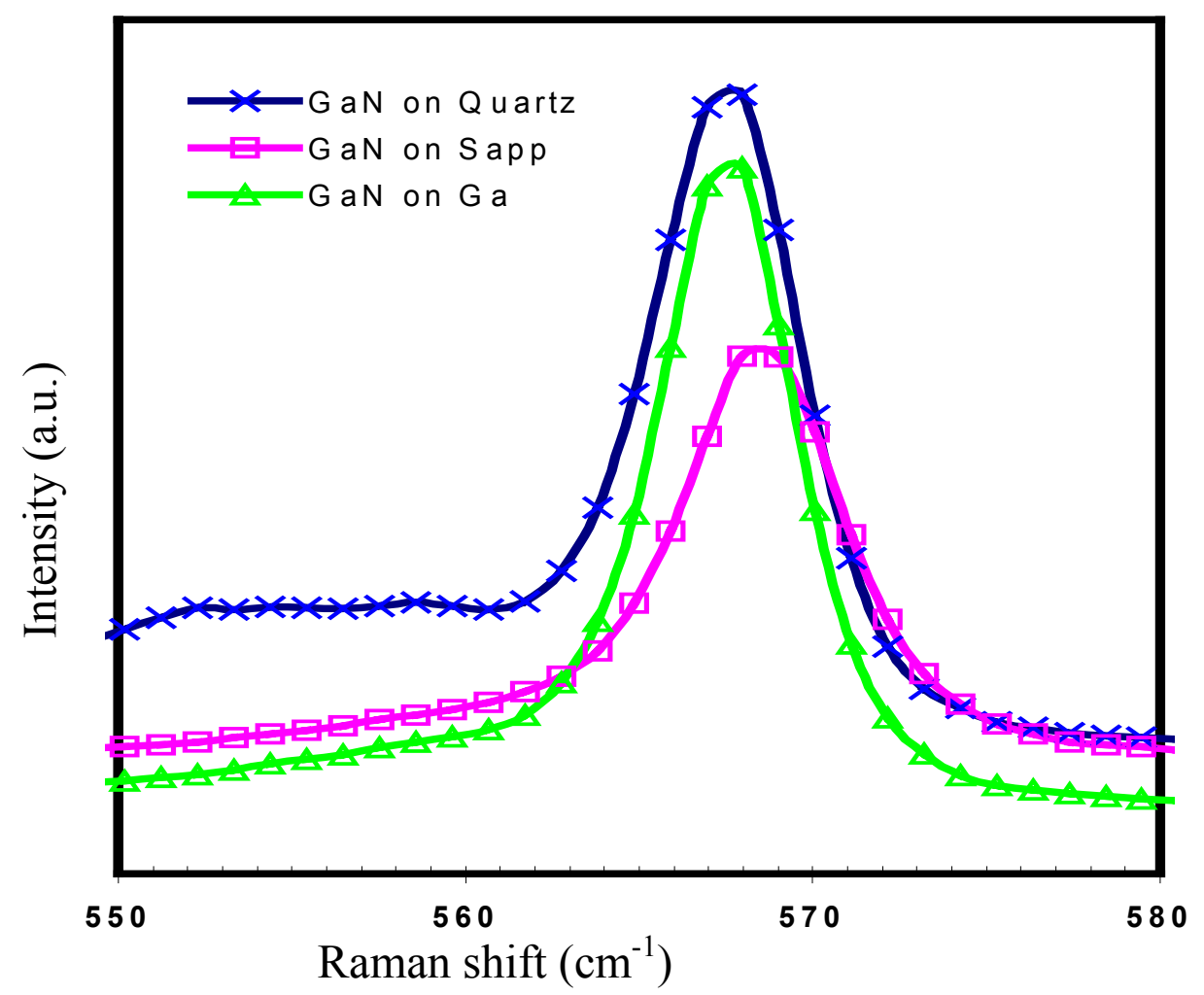

Figure $5.17 \mathrm{E}_{2}^{2}$ phonon frequencies of $\mathrm{GaN}$ films grown directly on molten $\mathrm{Ga}$; amorphous quartz; single crystalline sapphire. The results indicate that GaN grown on molten Ga is stress-free. 


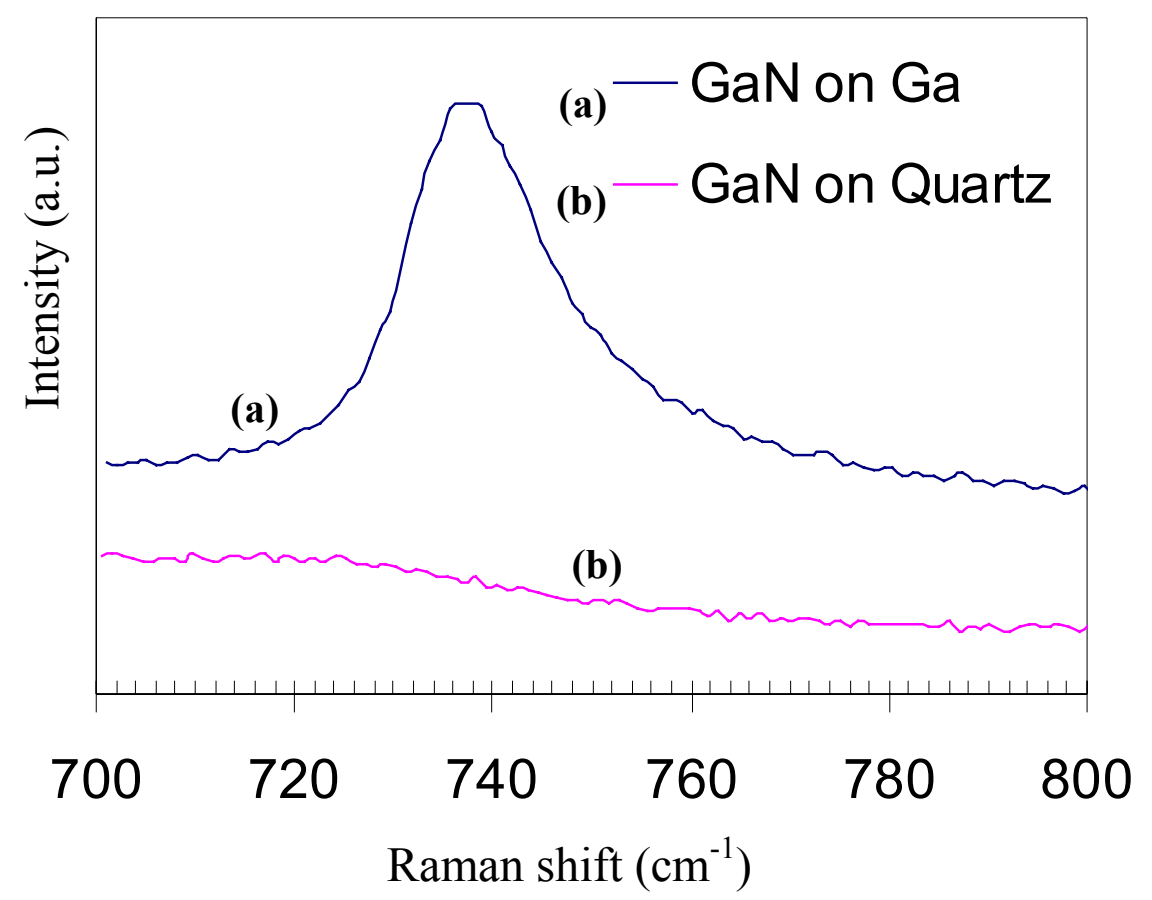

Figure 5.18 Profile of LO-phonon-plasmon coupled mode for GaN films grown directly on molten Ga and on amorphous quartz. The results indicate low native carrier concentration with GaN film grown on molten $\mathrm{Ga}$.

\subsubsection{Orientation of GaN Film on Molten Ga}

\subsubsection{XRD Texture Analysis over Large Area Film}

The XRD texture analysis was performed using a Bruker GADDS system with an area detector to determine both phase and the overall orientation information of textured GaN films. The spot size of X-ray source in the system is about few $\mathrm{mm}$ and the penetration depth is about $100 \mu \mathrm{m}$. The scans taken using the area detector are read as three-dimensional plots, where the horizontal direction in conical cross-sections represents Bragg angle $2 \theta$, the vertical direction $\chi$ is the tilt with respect to the plane represented by the X-ray source and the detector. The color contours represent the scattering intensity of the scan. Fig. 5.19(a) shows the plot centered at $2 \theta=34.6^{\circ}, \chi=$ 
$90^{\circ}$ for a $5 \mathrm{~mm} \times 5 \mathrm{~mm} \mathrm{GaN}$ free standing flake grown on top of Ga after dissolution of excess Ga. The $\chi$ integration of the (0002) reflection line from plot is shown in Fig. 5.19 (c). The presence of strong (0002) reflection indicates c-plane oriented wurtzite GaN. A small reflection of (10-11) is attributed to the presence of some skewed crystals in the films as shown in the SEM images. (See Fig. 5.19 (b)). The $2 \theta$ integration of the (0002) reflection line in Fig. 5.19 (d) indicates the tilt of the crystalline domains with respect to the normal, c-axis, is about $2.2^{\circ}$ determined from the full wave at half maximum (FWHM) of the curve. 

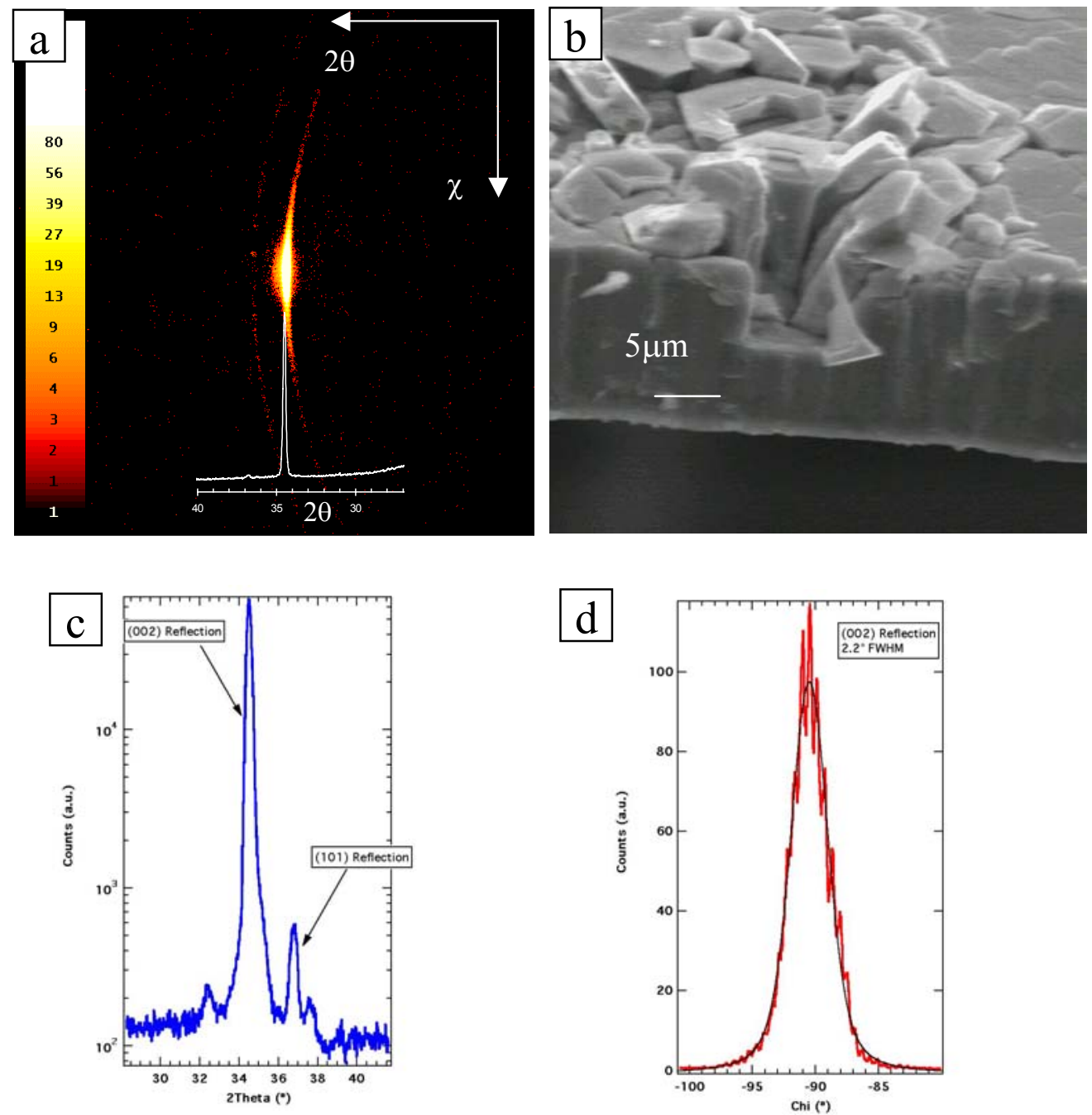

Figure 5.19 (a) XRD texture analysis of a $5 \mathrm{~mm} \times 5 \mathrm{~mm}$ free standing GaN flake; (b)

SEM image showing the presence of skewed crystals in certain regions within the film which cause the presence of (10-11) reflection in the plot showing in (a); (c) $\chi$ integration of the scan indicates preferential c-plane orientation; (d) $2 \theta$ integration of the (0002) reflection line showing a FWHM of $2.2^{\circ}$.

\subsubsection{TEM Studies to Determine Orientation between Individual Crystals}

XRD texture analysis only gives the overall orientation information of the large area $\mathrm{GaN}$ film. The detailed orientation information between neighboring crystals could not be resolved from the XRD texture analysis described in the previous section. 
Therefore cross sectional TEM analysis was done to study the orientation relationship between neighboring grains.

\subsection{Cross Sectional TEM Analysis}

Fig. 5.20 shows a bright field TEM image from a cross-section of the GaN film grown directly on Ga. The images were obtained with the $2^{\text {nd }}$ grain in zone axis of $<11 \overline{2} 0>$. The contrast observed clearly shows the existence of grain boundaries with mis-orientation between two adjacent crystals. Columnar growth of GaN crystals could be seen and no dislocation crops are found inside individual grains. However, the crosssectional image shows contrast for possibly two different growth modes: growth downwards with basal attachment and another growth mode using epitaxy from top, as indicated in Fig. 5.20. The alignment of crystals seemed to be set during film formation in the initial stages of nucleation and growth which probably occurred at region indicated by the dashed line. For growth upwards, Ga was supplied to the top through the cracks within the GaN film and spread quickly over the entire region of top surface. On the other hand, the basal growth seemed to stop after few microns of growth as evidenced in several experiments with different nitridation time scales. Note, after each of the successful experiment had Ga remaining at the bottom of the self-oriented GaN film.

Shown at right is a selected area diffraction pattern of the $2^{\text {nd }}$ grain in Fig. 5.20.

The diffraction pattern with a zone axis of [11 $\left.\overline{2}^{-} 0\right]$ indicates single crystal quality of the film. The (0001) diffraction spot is seen in the pattern due to double diffraction resulting with the particular zone axis of $\left[\begin{array}{lll}1 & \overline{2} & 0\end{array}\right]$. This was also confirmed in the dark field imaging analysis of (0001) diffraction spot. 

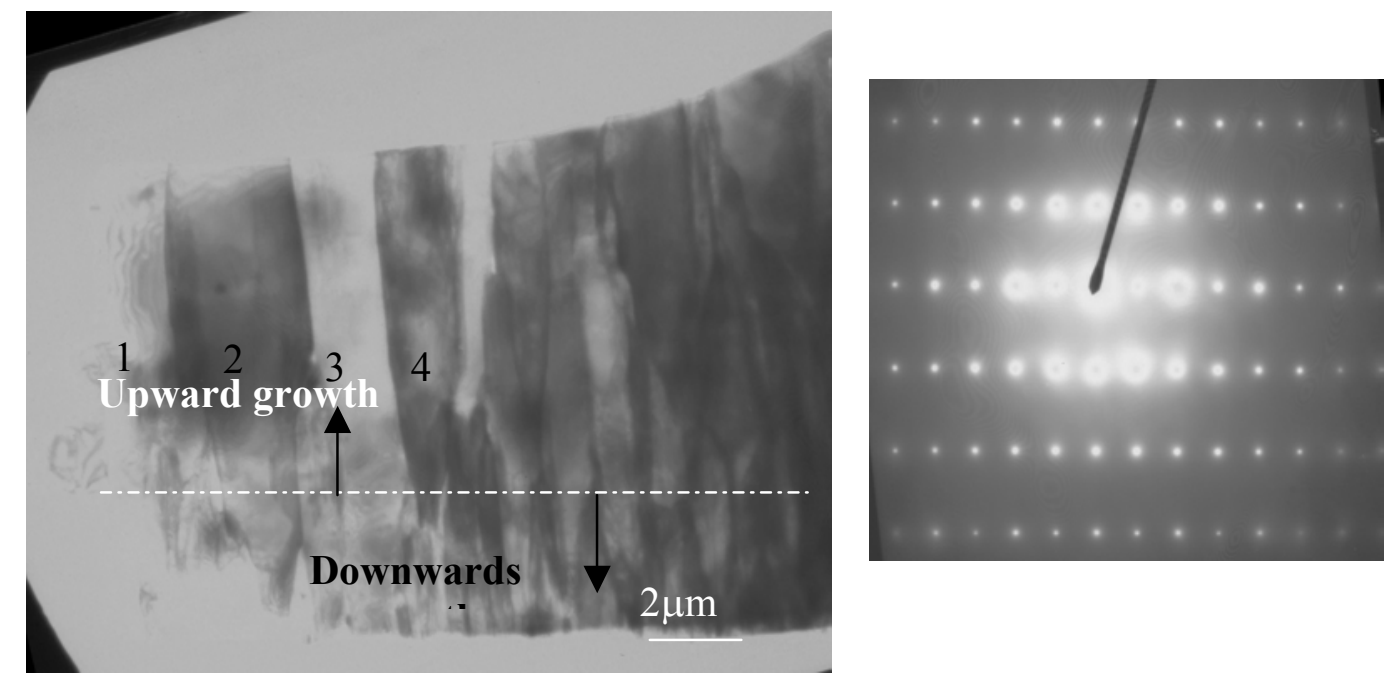

Figure 5.20 (a) TEM bright field image of a cross-section of GaN film with $2^{\text {nd }}$ grain on $\mathrm{ZA}<11 \overline{2} 0>$ showing contrast resulting from misorientations of few grains. (b) The selected area diffraction with ZA [11 $\left.2 \overline{2}^{-} 0\right]$ on the right taken from the $2^{\text {nd }}$ grain indicates single crystal of GaN.

\subsection{Kikuchi Line Analysis}

In order to quantify the misorientation between the grains, the method of Kikuchi lines using convergent beam electron diffraction (CBED) was performed since the Kikuchi lines are very sensitive to the orientation differences between grains [Yao, Y.\& Tholen, A., 2002; Gemperle, A. \& Gemperlová, J., 1995]. The Kinematical SADP Electron Diffraction Simulation program available online at WebEmaps [J. M. Zuo, J. C. Mabon, UIUC] was used to determine theoretical CBED patterns with different $\mathrm{X}$ and $\mathrm{Y}$ axis tilts of the grains. The comparison between the simulated CBED patterns using assumed tilt and twist angles and the experimentally obtained CBED patterns allowed us to determine the mis-orientation among grains within the cross-sectional sample. The CBED patterns were obtained from the top part of the grains numbered from 1 to 4 
shown in Fig. 5.20 within the cross-sectional sample and are compared with the corresponding simulated patterns. See Fig. 5.21. CBED pattern from grain 1 was taken on zone axis of [ 1120$]$ and others were taken by just moving the grain to the convergent beam. The c-plane twist angle between grain 1 and grains 2,3 and 4 are $8^{\circ}, 3^{\circ}, 9.7^{\circ}$, respectively. The tilt of c-axis for grains 2,3 and 4 with respect to grain 1 is about $0.2^{\circ}$,$0.4^{\circ}$ and $0.1^{\circ}$, which are very small and can be seen from the slightly off-symmetrical CBED pattern.
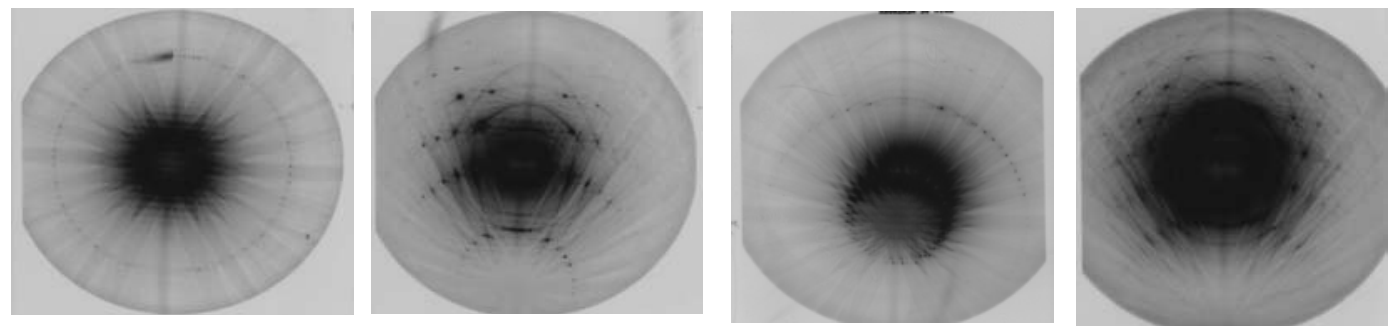

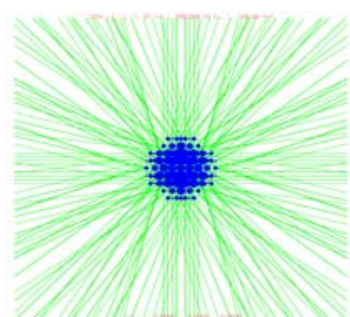

(1)

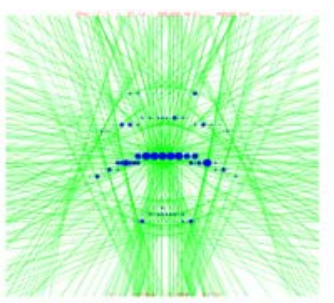

(2)

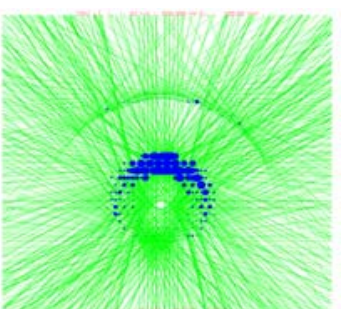

(3)

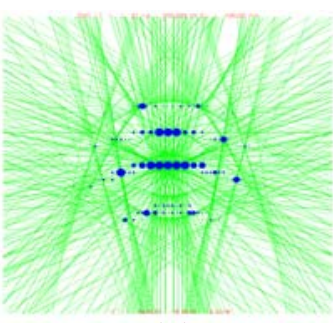

(4)

Figure 5.21 Experimental and simulated kinematical Kikuchi lines using CBED patterns from four grains with the following misorientation: (1) on ZA [11 20$]$; (2) $8^{\circ}$ twist, $0.2^{\circ}$ tilt (3) $3^{\circ}$ twist, $-0.4^{\circ}$ tilt; (4) $9.7^{\circ}$ twist, $0.1^{\circ}$ tilt; with respect to grain 1 .

\subsection{High Resolution TEM of Grain Boundaries}

HRTEM micrographs were obtained to understand the structure of the two kinds of boundaries observed. One is a boundary which has almost no misorientation as shown in Fig. 5.22(a). The CBED patterns shown in the insets for the two grains with no 
misorientation also confirm the perfect attachment. It was found that there is an intermittent layer of disordered GaN in-between the two adjacent crystals (Fig. 5.22 (b)). The width of the disordered layer ranges from $2 \mathrm{~nm}$ to $5 \mathrm{~nm}$ except at both ends of the boundary. The width of the layer at the top of the boundary is around $25 \mathrm{~nm}$ and it was not found at the bottom as shown in Fig. 5.22 (c). The EDX line scan under STEM mode cross the boundary in Fig. 5.23 shows the $\mathrm{N}$ concentration is apparently lower at the disordered layer. The variations of the growth conditions at the boundary might have caused differences in the local concentration of reactants. The disordered nitrogen deficient layer formed when there was more gallium around the boundary.
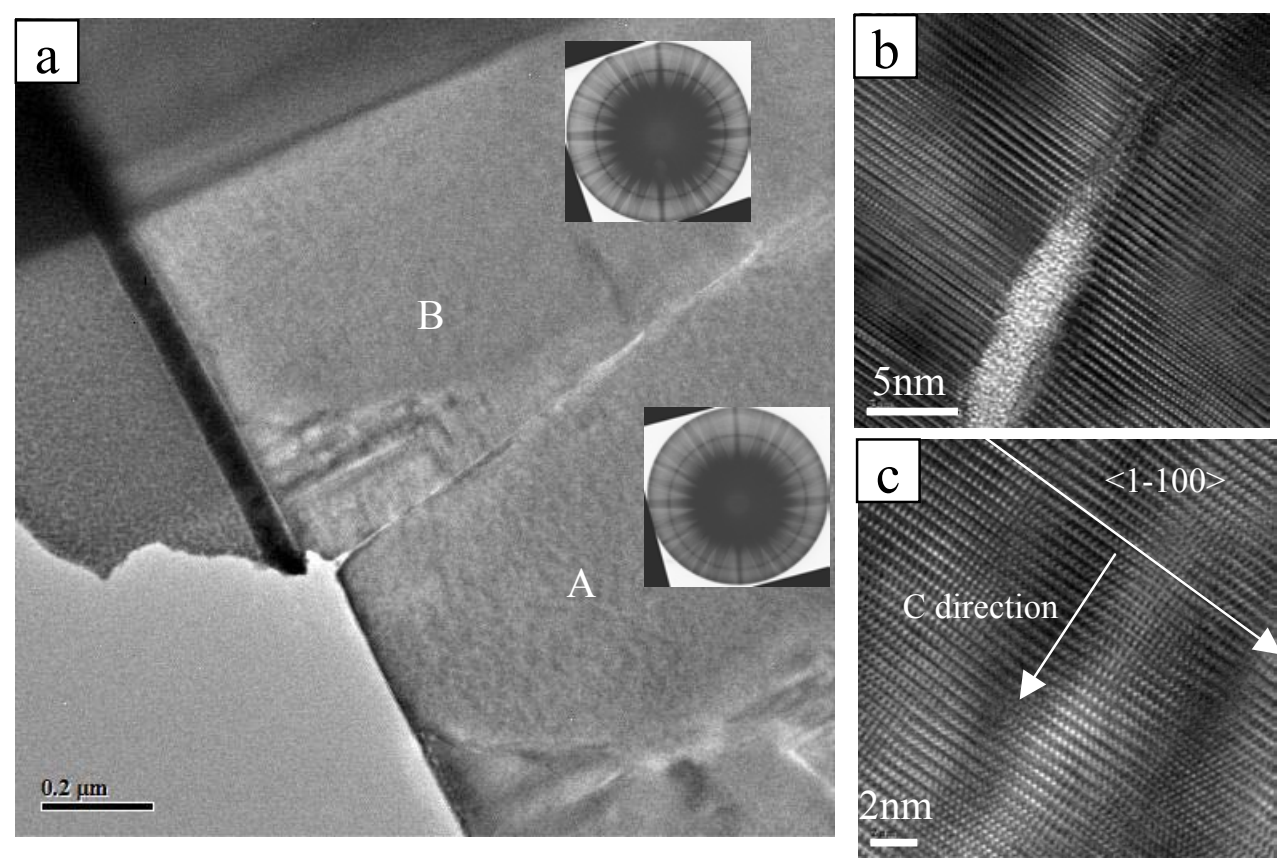

Figure 5.22 (a) A boundary with no misorientation. Insets show the corresponding CBED patterns for the two grains forming the boundary; (b) HRTEM image of the boundary shows the intermittent disordered layer at the boundary; (c) HRTEM image of the bottom part of the boundary shows no disordered layer. 


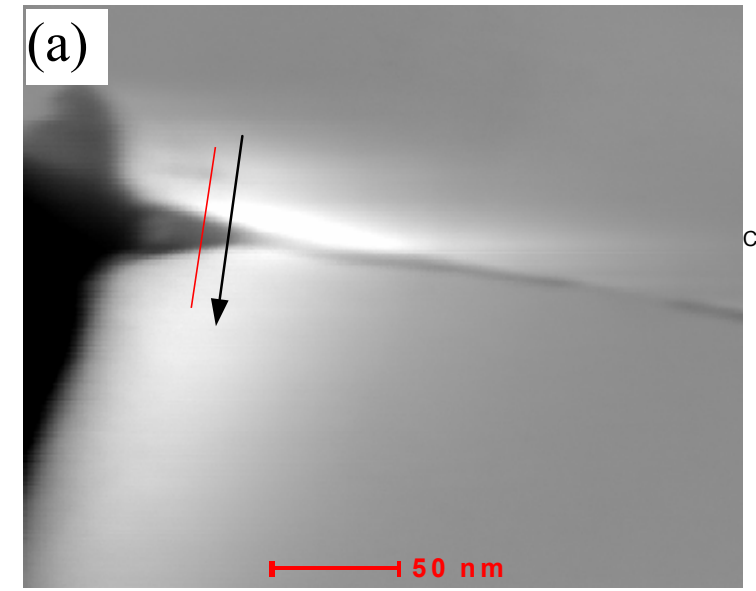

\section{(b)}

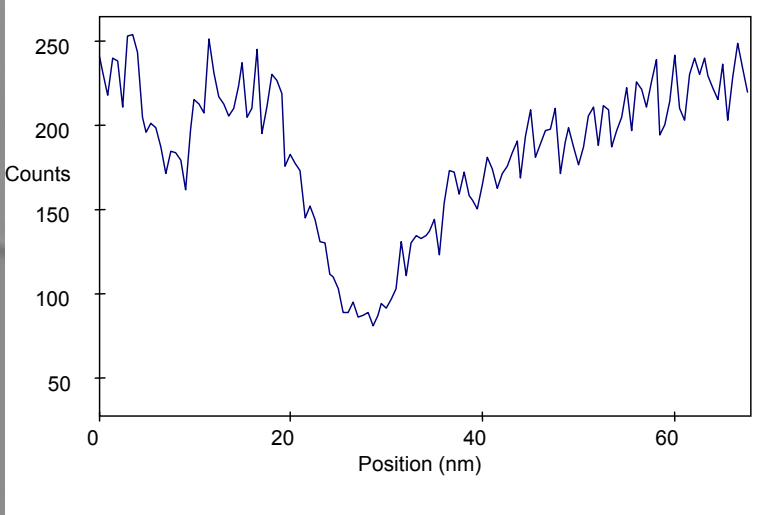

Figure 5.23 (a) STEM image of the boundary, the line indicates the region for EDX line scan; (b) N concentration profile along the boundary shown in (a), dwell time $15 \mathrm{~s}$, resolution $0.5 \mathrm{~nm}$.

Fig. 5.24 (a) shows the top part of the boundary between grains 2 and 3 shown in Fig. 5.20 that has a misorientation of $5^{\circ}$ twist in c-plane and $0.6^{\circ} \mathrm{c}$-axis tilt with respect to each other. The grain 2 is on zone axis $<11 \overline{2} 0>$ and grain 3 is off zone axis which can be seen that only 1-D lattice showed up in the HRTEM images. The boundary is straight, sharp and no disordered layer was found. Another boundary between grains 3 and 4 in Fig. 5.20 shows facets at the top region. The angle between the facet and c-plane was found to be around $61^{\circ}$, which is close to the angle between $(1 \overline{101)}$ and (0002). 


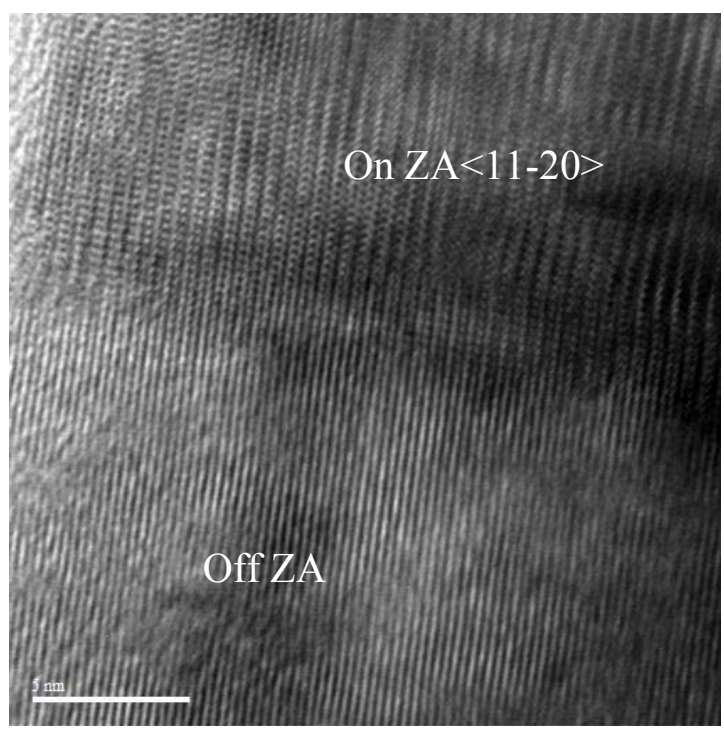

(a)

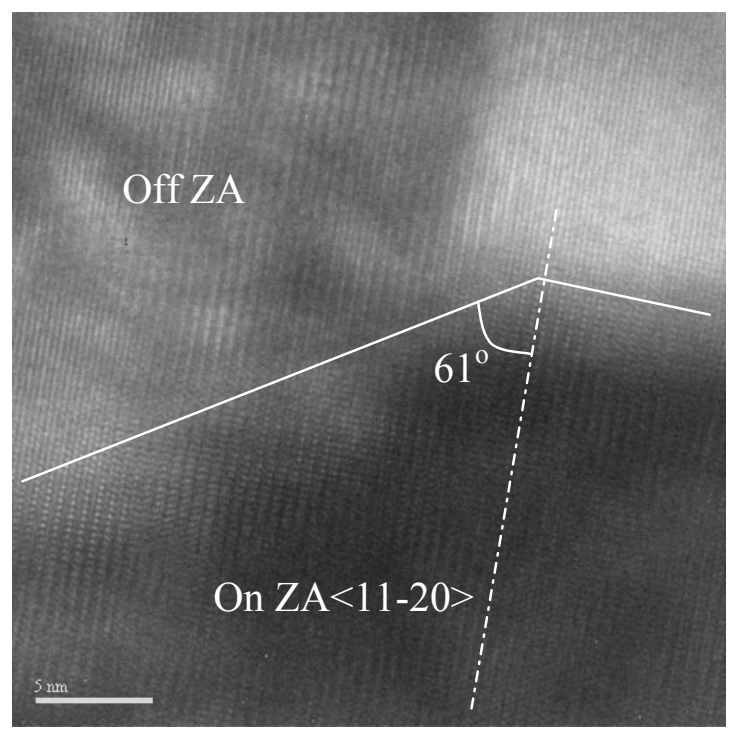

(b)

Figure 5.24 (a) HRTEM image of the straight boundary between grains 2 and 3 shown in

Fig. 5.20 with a misorientation of $5^{\circ}$ twist in c-plane and $0.6^{\circ}$ c-axis tilt; (b) HRTEM image of the (1 $\overline{1} 01)$ faceted boundary between grains 3 and 4 shown in Fig. 5.20.

In summary, the alternate grains seem to be closely oriented with respect to each other while adjacent grains seem to be mis-oriented with respect to each other by about 510 degrees of c-plane twist. At the same time, the single crystal SAD pattern indicated that there is some sort of self-orientation between crystals within these films with some imperfect attachment and rotational alignment of crystals with respect to each other during growth.

\subsubsection{Photoluminescence of Oriented GaN}

The photoluminescence spectra of the GaN crystals grown on Ga in Fig. 5.25 were obtained using $325 \mathrm{~nm} \mathrm{He}-\mathrm{Cd} \mathrm{UV}$ laser at room temperature. There is only a strong near band edge emission centered at $3.39 \mathrm{eV}$ with a FWHM of $0.13 \mathrm{eV}$ for the $\mathrm{GaN}$ directly grown on top of molten Ga. The band gap matches very well with the literature for wurtzite GaN [Levinshtein, et al. 2001]. But two peaks were found for the GaN 
grown on quartz substrate without the Ga buffer layer. One is centered at $3.32 \mathrm{eV}$, the other is centered at $3.4 \mathrm{eV}$. The split of the peaks in the $\mathrm{UV}$ region is believed to be caused by the high angle tilted boundaries or the rough surface of the $\mathrm{GaN}$ on quartz as shown in Fig. 5.14(b).

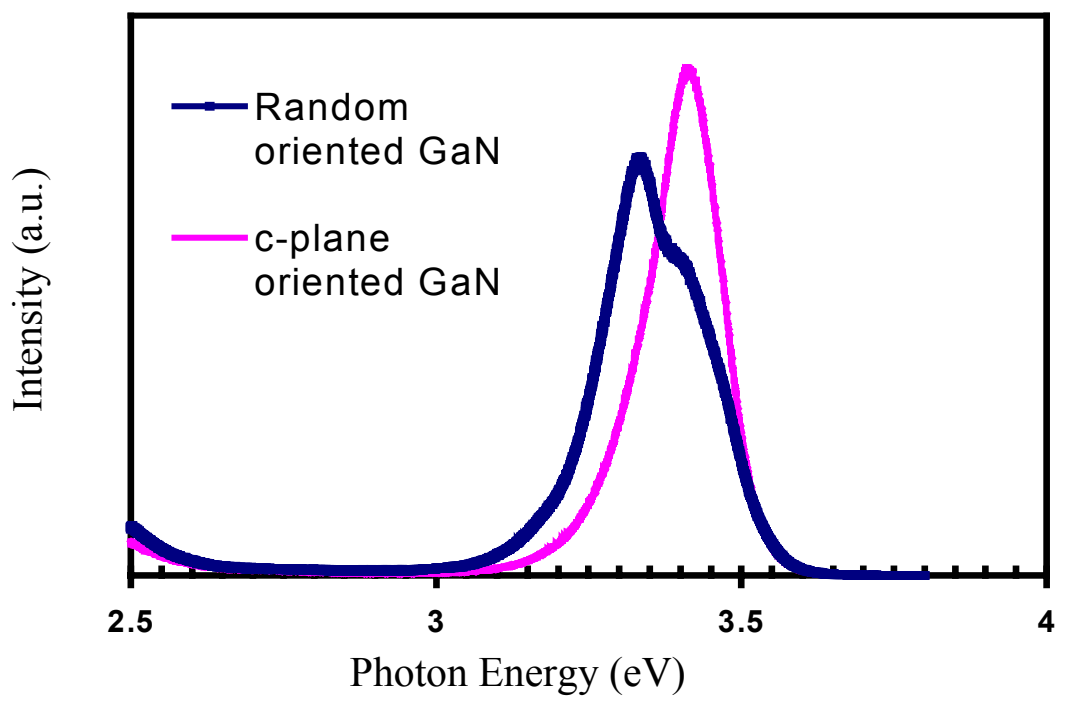

Figure 5.25 UV photoluminescence of GaN grown on $\mathrm{Ga}$ and on amorphous quartz substrates showing defects related luminescence for GaN directly grown on quartz.

The visible photoluminescence of $\mathrm{GaN}$ grown on $\mathrm{Ga}$ at room temperature was shown in Fig. 5.26. A broad peak centered at $2.2 \mathrm{eV}$ which is known as yellow luminescence appeared. The presence of yellow luminescence might be attributed to the grain boundaries between the individual GaN grains or $\mathrm{N}$ vacancy [DeMierry, P., et al., 1996]. Though the intensity of the yellow peak is about 1.5 stronger than the intensity of band edge emission at $3.4 \mathrm{eV}$, it still compares very well with the PL of GaN crystals grown under high pressure, high temperature whose yellow peak was about 2 time stronger than near band edge emission at $80 \mathrm{~K}$ [Krukowski, S., 1999]. 


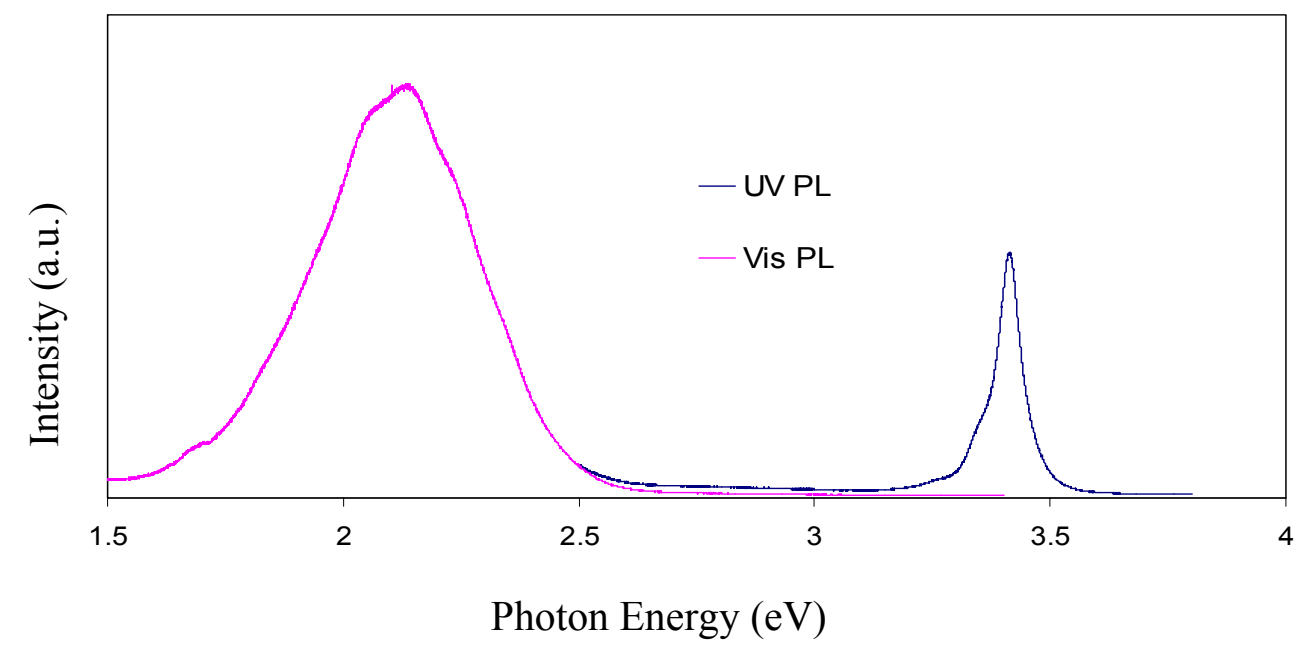

Figure 5.26 Visible and UV PL of GaN on Ga showing yellow luminescence centered at $2.2 \mathrm{eV}$ which might originates from $\mathrm{N}$ vacancies at the grain boundaries.

\subsubsection{Large Area Self-Oriented Film}

The oriented flakes as large as over $25 \mathrm{~mm}^{2}$ had been obtained from the experiments. Their optical images are shown in Fig. 5.27 (a-b). These flakes were translucent as shown in the optical images and were about $5 \mu \mathrm{m}$ thick. The power XRD spectrum and $\chi$ integration of the (0002) reflection of the flakes in Fig. 5.27 (c) \& (d) showed strong preferential orientation. 

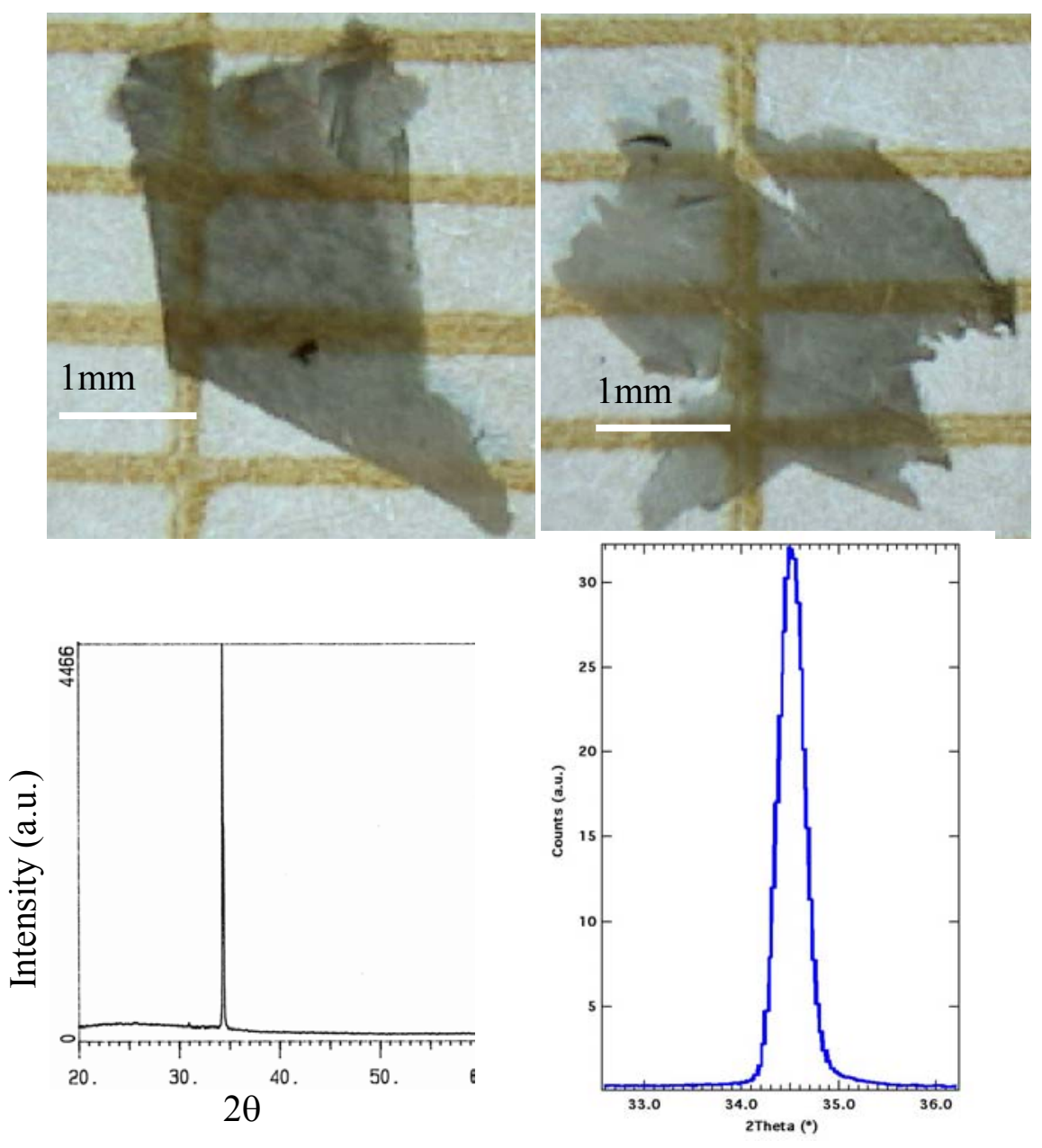

Figure 5.27 Optical images and XRD spectra of large area oriented flakes.

\subsection{Summary}

The oriented GaN flakes as large as $5 \mathrm{~mm} \times 5 \mathrm{~mm}$ has been synthesized demonstrating the concept of self-oriented growth of GaN films. The growth occurred with nitridation of molten Ga films using atomic nitrogen at low pressure. Raman spectrum indicates GaN on gallium is free from stress and has low native donor concentration on the order of $10^{17} / \mathrm{cm}^{3}$. XRD texture analysis of the over $25 \mathrm{~mm}^{2}$ free standing gallium nitride flake showing only reflections of (0002) and (0004) planes gives 
an overall c-axis tilt of $2.2^{\circ}$. Cross-sectional TEM micrographs showed that the resulting GaN film did not contain dislocation crops inside the grains but diffraction contrast due to small misorientation between them. The analysis of Kikuchi lines in convergent beam electron diffraction patterns gave misorientation of about $5-10^{\circ} \mathrm{c}$-plane twist and less than $1^{\circ} \mathrm{c}$-axis tilt between the neighboring column grains. HRTEM micrographs of one grain boundary with almost no misorientation showed an intermittent, nitrogen deficient disordered GaN layer, while the boundary with some twist and tilt showed sharp, misoriented interface. PL spectrum with $325 \mathrm{~nm} \mathrm{He}-\mathrm{Cd}$ laser showed a near band edge emission of $3.39 \mathrm{eV}$ at room temperature. 


\section{CHAPTER 6}

\section{DIRECT SYNTHESIS OF GALLIUM NITRIDE NANOWIRES WITH CONTROL ON GROWTH DIRECTIONS}

\subsection{Introduction}

Nucleation and growth of $\mathrm{GaN}$ from molten gallium with pure nitrogen plasma have produced 2-D crystal growth due to wetting properties of gallium under growth conditions as described in the previous chapters. Changing of wetting properties of $\mathrm{Ga}$ with changing gas phase environment has also been shown for favoring 1-D nanostructures growth with spontaneous nucleation from low melting melts [Sharma \& Sunkara, 2002]. GaN bulk crystals has wide applications in opto-electronics, while defect-free 1-D nanostructures are similarly of great interest for nanoelectronics. The miniaturization of electronic devices necessitates development of materials with sizes less than $50 \mathrm{~nm}$. In addition, the quantum effects with reduced dimensions for solids also give rise to new properties for materials with diameters below a certain size, such as the Bohr exciton radius [Moriarty, P., 2001]. Nanowires have been found to be promising as building blocks for nanoelectronics [Huang, Y., Lieber, C.M., et al., 2002]. Wurtzitic GaN nanowires are specifically of interest in opto-electronic applications as waveguides [Johnson, J.C. et al., 2002] and lasers [Johnson, J.C., et al., 2003].

So far, GaN nanowires have been primarily synthesized using either catalyst [Chen, C.C. \& Yeh, C.C., 2000] or oxide assisted techniques [Shi, W.S., Lee, S.T., et al., 2001]. In the case of anisotropic crystals like wurtzite GaN, GaN exhibits direction 
dependent properties [Feng, D.P., et al., 1999; Waltereit, P. et al., 2000]. So, it is important to be able to synthesize $\mathrm{GaN}$ nanowires with control on growth directions. Recently, control on the growth directions of $\mathrm{GaN}$ nanowires have been achieved using hetero-epitaxy with different single crystal templates mediated through catalyst clusters [Kuykendall, T., et al., 2004], but this has not been shown in direct nitridation schemes.

Our earlier studies showed that low-melting metal melts, such as Ga, could be used as medium for spontaneous nucleation and basal growth of one-dimensional nanostructures for Si and other materials [Sunkara, M.K., et al., 2001; Sharma \& M. K. Sunkara, 2004]. This spontaneous nucleation and basal growth for 1-D crystals also apply directly to $\mathrm{GaN}$ precipitation from Ga melts, as the solubility of nitrogen in $\mathrm{Ga}$ is quite small [Karpinski, J., et al., 1984]. In fact, the direct reaction of Ga with nitrogen plasma [Chandrasekaran \& Sunkara, 2002], or dissociated ammonia [He, M., et al., 2001] have already been shown to result in $\mathrm{GaN}$ nanowires. The growth directions of the resulting nanowires from these studies were different, i.e., $<10-10>$ in the case of nitrogen plasma at higher temperatures [Chandrasekaran \& Sunkara, 2002] and $<11-20>$ direction in the case of direct reaction with ammonia [He, M., et al., 2001]. Both these two directions are perpendicular to the non-polar surfaces of wurtzite $\mathrm{GaN}$ and the synthesis of $\mathrm{GaN}$ wires with $<0001>$ direction, i.e., the polar surface normal is still elusive. Furthermore, it is still not clear on what factors control the one-dimensional growth with spontaneous nucleation of $\mathrm{GaN}$ from Ga melts with direct reactions. This is because of our lack of basic understanding about both nucleation and growth mechanisms.

Here, we studied GaN nanowires synthesis with controlled growth directions by varying growth conditions alone, rather than relying on hetero-epitaxial growth onto 
single-crystal substrates. This is very useful in future implementation of nanowires in practical applications.

\subsection{Experimental}

Two types of nitridation experiments were performed: (i) direct nitridation of molten Ga melt in either disassociated ammonia or nitrogen/hydrogen plasma and (ii) vapor transport of $\mathrm{Ga}$ in dissociated ammonia. The experiments were performed in an 8inch diameter water-cooled double wall stainless steel vacuum chamber equipped with an ASTeX $^{\circledR}$ Model AX4500 ECR plasma source. The setups of the substrates for both types of nitridation experiments are schematically shown in Figs. 6.1 (a) and (b). Amorphous quartz substrates were used throughout the study. Prior to every experiment run, the substrates were cleaned using hot $\mathrm{KOH}$, followed by ultrasonication in acetone, and then drying using argon gas. After loading the sample, the chamber was pumped to base pressure of 2 mTorr, baked and purged with nitrogen to remove any possible residual oxygen or $\mathrm{H}_{2} \mathrm{O}$. In the first setup, the quartz substrates were coated with a thin Ga film (approximately 20 microns thick) and placed on a ceramic-coated heater (Boroelectric ${ }^{\mathrm{TM}}$ from GE Advanced Ceramics). Initially, the Ga film was subjected to hydrogen plasma for 10 minutes at $830^{\circ} \mathrm{C}$ to remove any native oxide on the Ga melt. Hydrogen plasma treatment typically converted the Ga film into macroscopic Ga droplets of sizes ranging from sub-micron to tens of microns. Then the substrates were exposed to plasma containing different mixtures of hydrogen and nitrogen at a pressure of 50 mtorr and a temperature of $830^{\circ} \mathrm{C}$. Nitridation experiments were also performed by exposing the $\mathrm{Ga}$ covered quartz substrates directly to $50 \mathrm{sccm}$ of ammonia without the plasma at 20 Torr, 
$830^{\circ} \mathrm{C}$. In this case, the Ga-film-covered-quartz substrates were placed on a four-inch $\mathrm{SiC}$ susceptor that was radiatively heated uniformly by a tungsten coil filament.

The reactive vapor transport experiments were conducted using pre-synthesized polycrystalline $\mathrm{GaN}$ powder as the source of $\mathrm{Ga}$. The source was placed on the $\mathrm{SiC}$ susceptor as shown in Fig. 6.1 (b), and the substrate was placed at a distance of $2 \mathrm{~mm}$ from the Ga source. These experiments were performed at a pressure of 20 Torr with ammonia flow rate of $50 \mathrm{sccm}$. The substrate temperature was ramped up to $700{ }^{\circ} \mathrm{C}$ and then the flow of $\mathrm{NH}_{3}$ started while continuing the temperature ramping to $900{ }^{\circ} \mathrm{C}$ in about 10 minutes. The substrates were kept at 20 Torr, $900{ }^{\circ} \mathrm{C}$ for $1-2$ hours. After the nitridation at $900{ }^{\circ} \mathrm{C}$ for $1-2$ hours, the experiment was shutdown by ramping the temperature down to $700{ }^{\circ} \mathrm{C}$ in 10 minutes, followed by replacing the $\mathrm{NH}_{3}$ flow with $\mathrm{N}_{2}$. Finally, the substrate was cooled down further under flowing nitrogen to room temperature. In all cases, the temperature was measured using a C-type thermocouple which was calibrated earlier using aluminum (melting point of $660^{\circ} \mathrm{C}$ ) and germanium (melting point of $937^{\circ} \mathrm{C}$ ).

The synthesized $\mathrm{GaN}$ samples were characterized using scanning electron microscopy (SEM, LEO SUPRA ${ }^{\mathrm{TM}}$ 35VP), x-ray Diffraction (XRD, RigakuVSDXR3000), high-resolution transmission electron microscopy (HR-TEM, JEOL 2010), Raman spectroscopy and photoluminescence (Renishaw InVia Raman-PL microscope). 

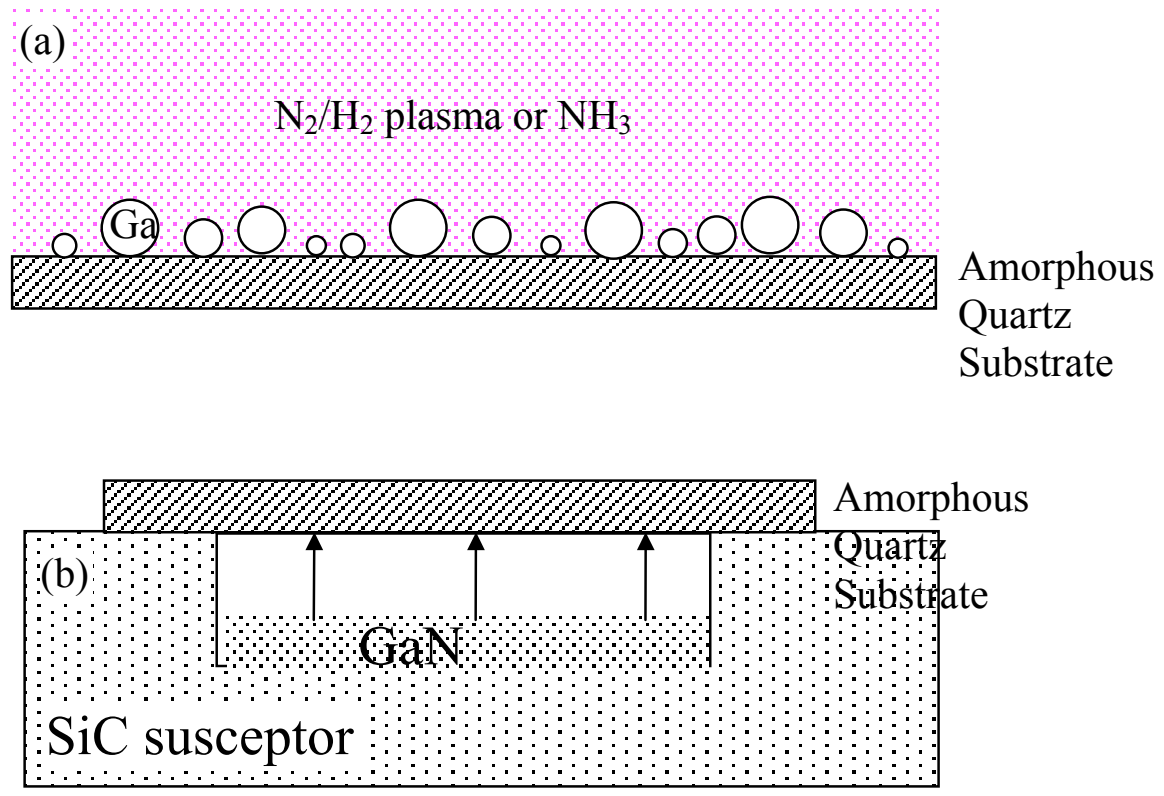

Figure 6.1 Experimental setup for (a) $<0001>\mathrm{GaN}$ nanowire synthesis from Ga droplets; (b) reactive vapor transport growth of $<10-10>\mathrm{GaN}$ nanowire in $\mathrm{NH}_{3}$ atmosphere.

\subsection{Result and Discussion}

\subsubsection{Growth of $<0001>$ GaN Nanowires}

The gallium films on the quartz substrates agglomerated into Ga droplets with a considerable size distribution during ramping up the temperature. SEM image of the gallium droplets in Fig. 6.2(a) shows sizes distribution from $150 \mathrm{~nm}$ to $50 \mu \mathrm{m}$.

Nitridation using pure nitrogen plasma resulted in the spreading of nitrogen saturated Ga droplets over the substrate and developed GaN crystalline crust on molten Ga similar to our results earlier [Li, H., et al., 2004]. However, the nitridation using plasma containing hydrogen/nitrogen at ratio (flow rate) ranging from 1:5 to 1:2 resulted in $\mathrm{GaN}$ nanowires formation from molten Ga droplets which could be clearly seen in Fig. 6.2(b). The nitridation of $\mathrm{Ga}$ droplets using ammonia, without the plasma, at $830^{\circ} \mathrm{C}$ also resulted in 
high densities of $\mathrm{GaN}$ nanowires. The diameters of the wires range from 15 to $50 \mathrm{~nm}$ and the average diameter was estimated to be $20 \mathrm{~nm}$ (See Fig.6.3). The length of the wires was about $25 \mu \mathrm{m}$ for the experiment lasting only half an hour.
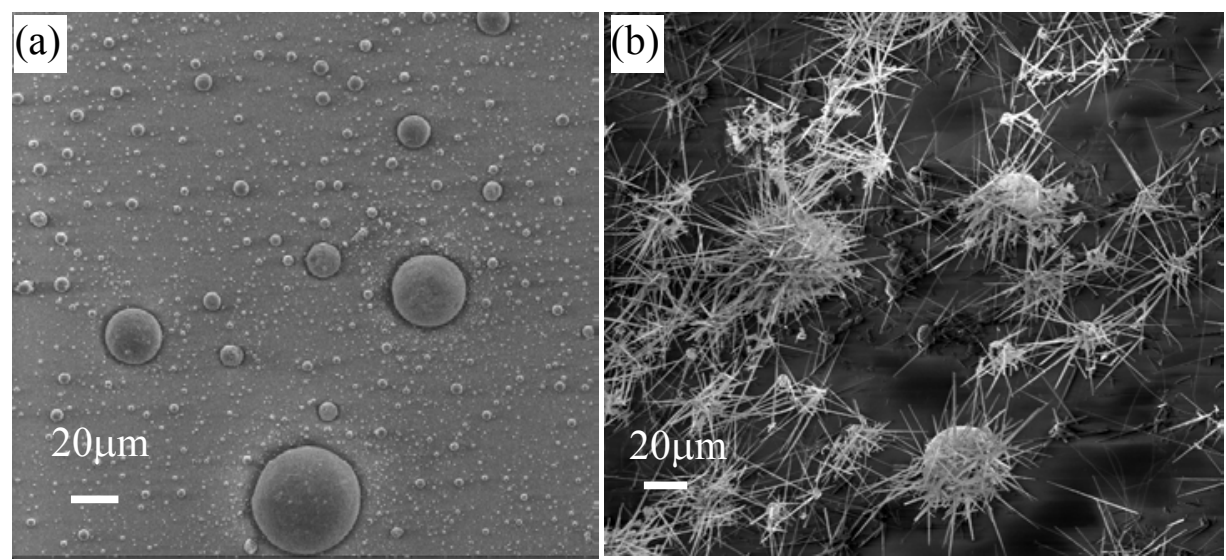

Figure 6.2 (a) Ga droplets with considerable size distribution on quartz substrates (b)

GaN wires on top of gallium droplets $\mathrm{H}_{2}$ and $\mathrm{N}_{2}$ plasma; conditions: $830{ }^{\circ} \mathrm{C}$, flow rate $\mathrm{H}_{2}: \mathrm{N}_{2}=1: 2, \mathrm{P}=50$ mtorr.

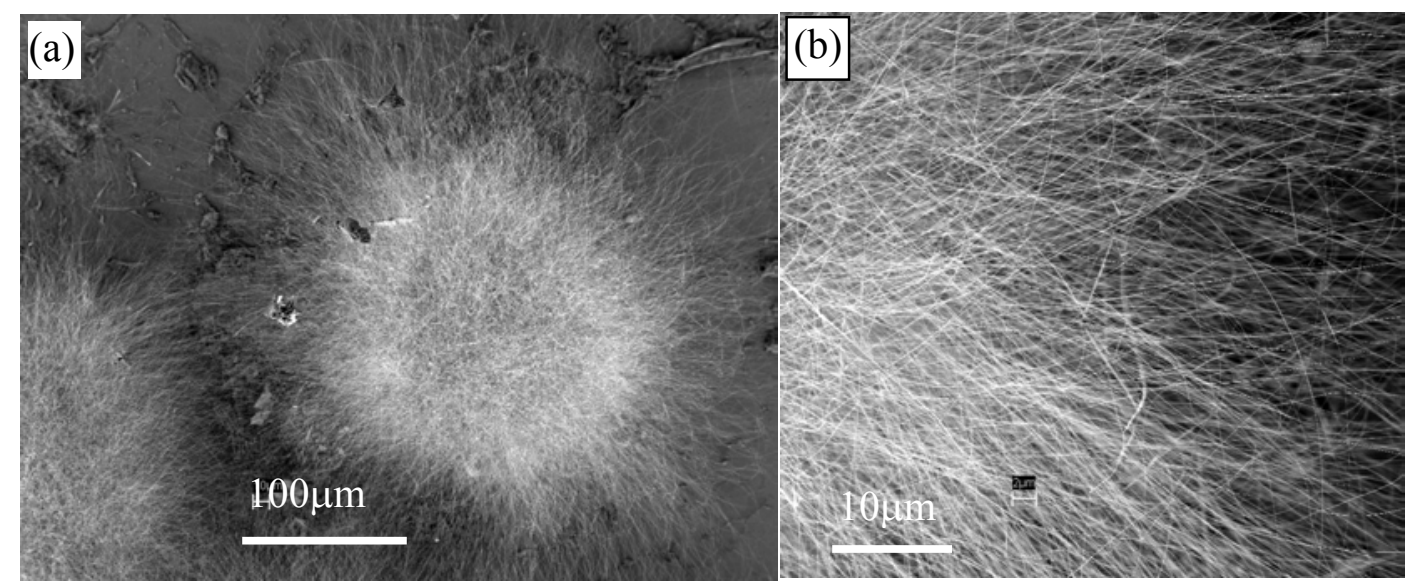

Figure 6.3 High density of GaN nanowire as thin as $15 \mathrm{~nm}$, up to hundred microns long from direct reaction between $\mathrm{Ga}$ and $\mathrm{NH}_{3}$.

TEM images of a $27 \mathrm{~nm}$ nanowire in Fig. 6.4 show that the nanowire is single crystalline with few stacking faults in (0001) planes. The growth direction of the nanowires is $<0001>$ and the distance between (0001) planes is found to be $5.2 \AA$, which 
is approximately the same as lattice constant of bulk wurtzite GaN, a=3.189 $\AA, c=5.185$ $\AA$. There is no oxide sheath at the edge of the nanowires. The FFT (fast Fourier transform) diffraction pattern of the nanowires lattice shown in the Fig. 6.4(c) shows a zone axis $(\mathrm{ZA})$ of $\langle 11-20\rangle$, indicating the direction normal to the growth direction is $<10-10>$.
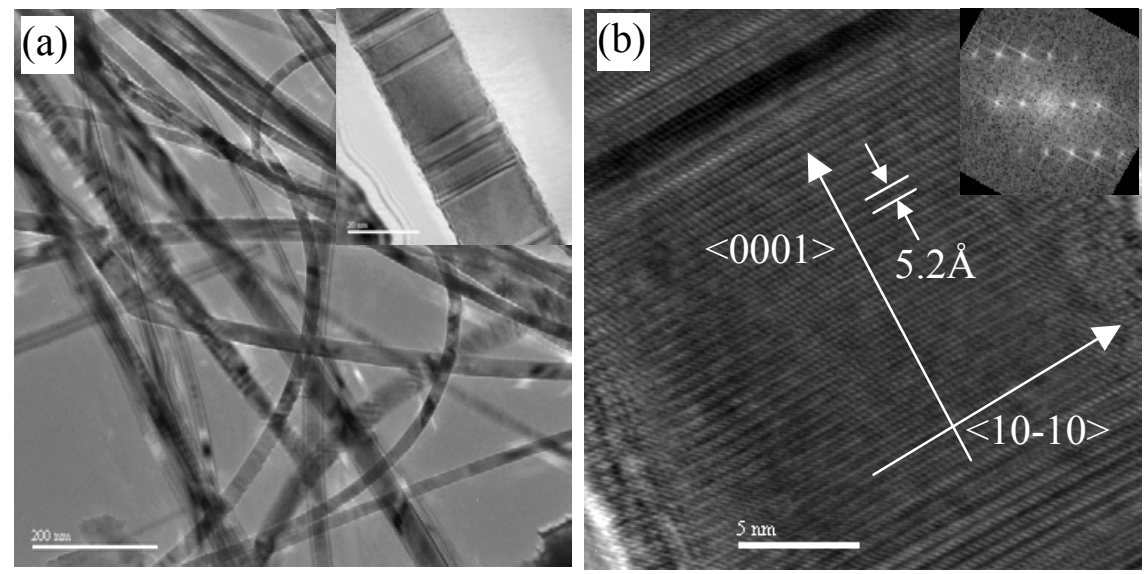

Figure 6.4 (a) TEM micrographs of $<0001>$ GaN nanowires, inserted is a $27 \mathrm{~nm}$ nanowires with stacking faults in c-plane along the wire; (b) HRTEM of GaN showing growth direction of $<0001>$ and no oxide sheath, inserted is the FFT pattern with ZA $<11-20>$.

The nitridation of Ga droplets using nitrogen plasma in the absence of hydrogen did not result in nanowire growth. Pure nitrogen plasma nitridation of molten Ga resulted in GaN crust with hexagonal platelet shaped crystals parallel to molten Ga surface. The addition of hydrogen to nitrogen plasma with a $\mathrm{H}_{2} / \mathrm{N}_{2}$ ratio of 1:5 to $1: 2$ resulted in multiple nanowires from each $\mathrm{Ga}$ droplet. The addition of hydrogen beyond a $\mathrm{H}_{2} / \mathrm{N}_{2}$ ratio of 1:1 did not even result in nitridation of $\mathrm{Ga}$ within the time period allowed. The presence of hydrogen in the gas phase increases the surface tension of the Ga surface and reduces the wetting with the GaN nuclei. Under the reduced wetting conditions, the 
lateral propagation of $\mathrm{GaN}$ nuclei is reduced, while the basal growth via dissolved nitrogen species leads to 1-D growth similar to our earlier results with Si nanowires from Ga droplet [Sunkara, M.K., et al., 2001]. The sketch of the growth processes for $<0001>$ $\mathrm{GaN}$ nanowires from Ga droplets is shown in Fig. 6.5. In this case, the nanowires are expected to grow in c-direction due to the expected alignment of basal (0001) planes of GaN crystal nuclei on molten Ga surface [Li, H., et al., 2005].
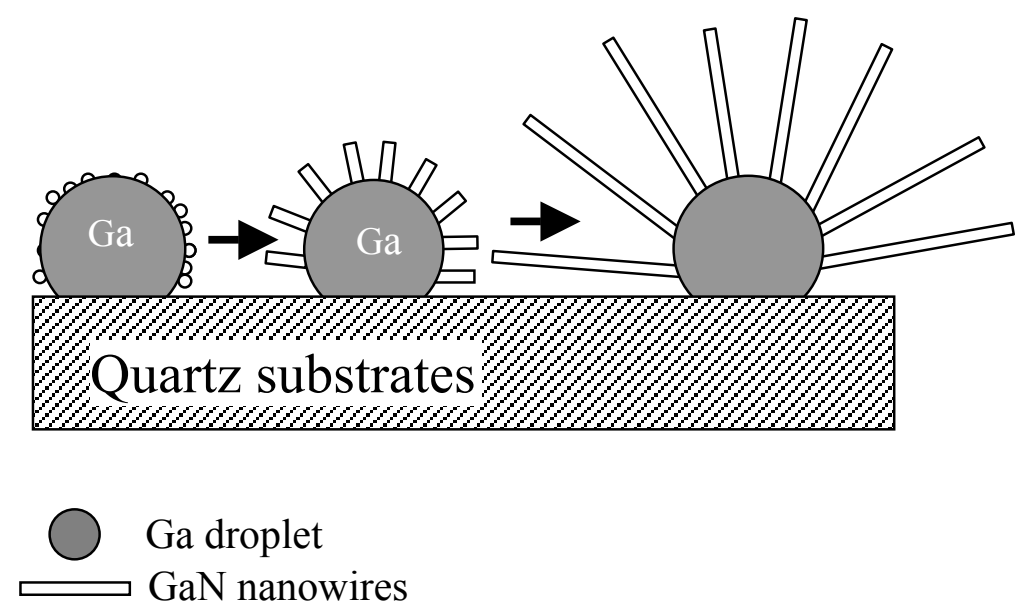

Figure 6.5 Sketch of $\mathrm{GaN}<0001>$ nanowires growth with basal growth from Ga melts.

\subsubsection{Growth of $<10-10>$ GaN Nanowires}

Experiments performed using controlled vapor transport of Ga in the presence of disassociated ammonia onto quartz substrates resulted in high density, sub $20 \mathrm{~nm}$ size GaN nanowires with lengths exceeding hundred microns. (See Fig. 6.6(a)) Here, the Ga vapor was supplied in a controlled manner by decomposing GaN powders kept on susceptor at $950{ }^{\circ} \mathrm{C}$. Major portion of the polycrystalline GaN powder used in the source still remained after the experiment. TEM images of a $17 \mathrm{~nm}$ wires is shown in Fig. 6.6 (b). The distance between the lattice planes along the growth direction in the HRTEM 
micrograph was $2.8 \AA$, which is close to the d-spacing of $\{10-10\}$ planes. The experiments using molten $\mathrm{Ga}$ as gallium vapor source led to a large amount of Ga vapor transport onto the quartz substrate and the nitridation results were similar to those obtained shown in Fig. 6.3(a).
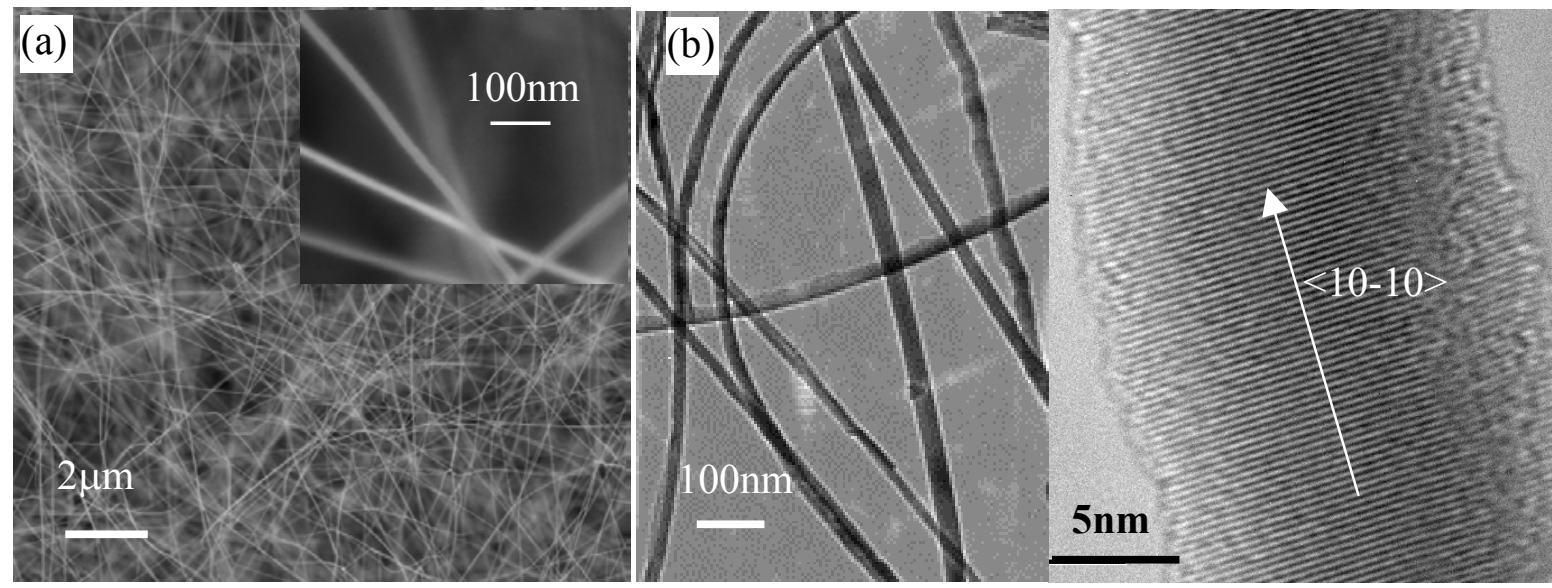

Fig. 6.6 (a) $<10-10>\mathrm{GaN}$ nanowires with average diameter of $25 \mathrm{~nm}$ using reactive vapor transport of $\mathrm{Ga}$ in the presence of $\mathrm{NH}_{3}$; (b) TEM micrographs of $<10-10>$ nanowires with no defects and oxide sheath.

In the vapor transport experiments using $\mathrm{GaN}$ as source for controlled Ga vapor, the underlying growth mechanism is quite different from the above-mentioned mechanism with high Ga amount in the initial stages. These experiments are similar to our recent work on InN nanowires using indium and ammonia [Vaddiraju, S., et al., 2005]. This study showed that InN crystal nucleation occurs first and then the 1-D growth occurs by liquid phase epitaxy through In droplets in a tip-led fashion. The schematic of the growth processes is shown in Fig. 6.7. In order to understand the interface between $\mathrm{GaN} / \mathrm{Ga}$, experiments were performed using polycrystalline $\mathrm{GaN}$ on quartz substrates. The results show preferential adsorption of Ga droplets on the edge planes of platelet 
shaped GaN crystals and nanowire growth preferentially from the edge planes of the GaN crystals. See Fig. 6.8. This preferential adsorption of Ga droplets on the sides of the hexagonal platelets will make the growth direction of the resulting nanowires to be either $<10-10>$ or $<11-20>$. Our investigations using HRTEM indicated that the most of the resulting nanowires have a growth direction of $<10-10>$ but not $<11-20>$. The Ga droplets at the tips of long and thin nanowires might have evaporated during shutdown and were not observed in our experiments.

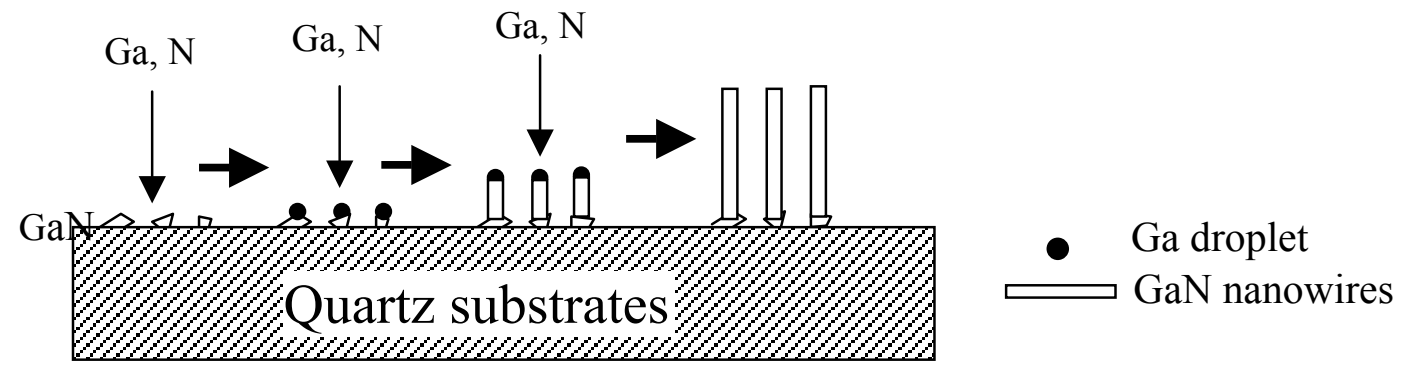

Figure 6.7 Sketch of $<10-10>\mathrm{GaN}$ nanowires growth from the GaN crystals through liquid phase epitaxy.

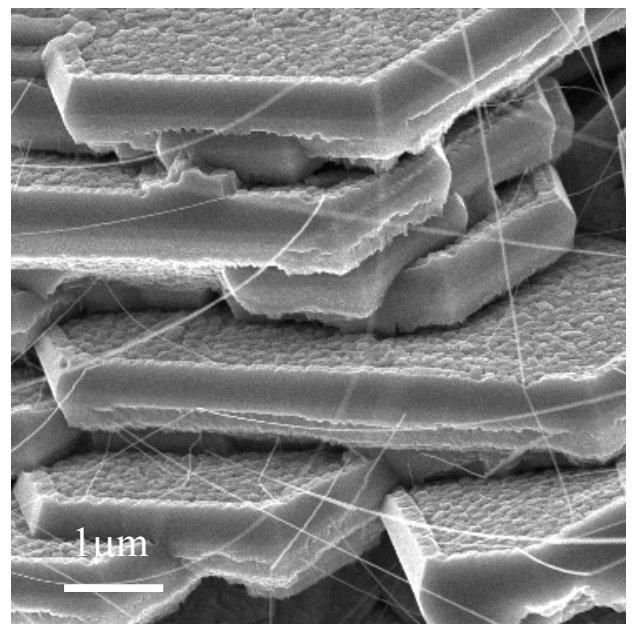

Figure $6.8 \mathrm{GaN}<10-10>$ nanowires grown from side facets of $\mathrm{GaN}$ hexagonal platelets using reactive vapor transport in the presence of $\mathrm{NH}_{3}$. 


\subsubsection{Optical Properties of GaN Nanowires}

The bandgap of GaN has been found to be dependent on the crystallographic orientation [Kuykendall, T., et al., 2004; Waltereit, P., et al., 2000]. The PL spectra of the GaN nanowires with two different growth directions, shown in Fig. 6.9, were obtained using excitation from a $325 \mathrm{~nm}$ He-Cd continuous wave (CW) UV laser with the sample at room temperature. Broad peaks centered from $3.1 \mathrm{eV}$ to $3.5 \mathrm{eV}$ were found with different laser power, and the peaks red-shift with the increasing laser power. A spectral dip is also apparent at $3.4 \mathrm{eV}$. With the small spot size involved $(\sim 10 \mu \mathrm{m} \times 10 \mu \mathrm{m})$, laserinduced heating likely occurs in the nanowires and results in the decreasing of bandgap of GaN. The bandgap variation with temperature for $\mathrm{GaN}$ follows the Varshini formula [Tiginyanu, I. M., et al., 2003]:

$$
\mathrm{E}(\mathrm{T})=\mathrm{E}_{0}-\mathrm{aT}^{2} /(\mathrm{T}+\mathrm{b})
$$

where $\mathrm{a}$ and $\mathrm{b}$ are known as Varshini's fitting parameters, $\mathrm{E}_{0}$ is the bandgap value of GaN at $0 \mathrm{~K}$. The spectral dip is suggested to come from the spectral response of the photoluminescence system. Assuming a Gaussian shape for the spectral dip, correcting the spectra, and fitting the resulting spectra to a Gaussian to obtain the peak of the spectral weight, the relationship of the bandgap of these nanowires with laser intensity is determined, as shown in Fig. 6.10. The bandgap of the $<10-10>$ nanowires blue-shifts about $50 \mathrm{meV}$ compared to the $<0001>$ nanowires, which is lower than that of $100 \mathrm{meV}$ observed in an early work [Kuykendall, T., et al., 2004]. The reasons could be due to the differences in surface facets and the diameters of the nanowires, as well as the temperature range for PL measurements. 

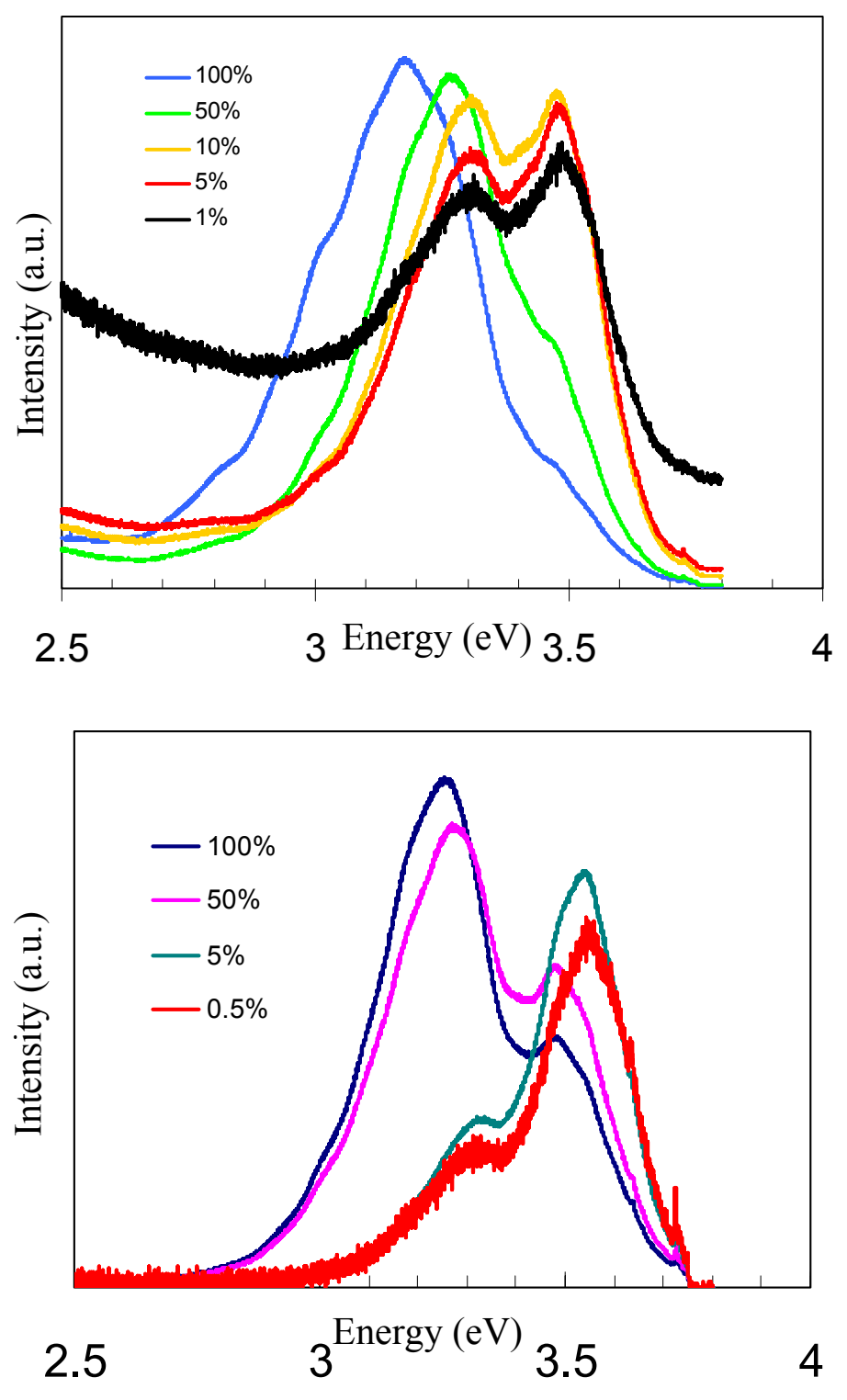

Figure 6.9 UV-PL of (a) $<0001>$ and (b) $<10-10>$ GaN nanowires with different power density showing red-shift with increasing laser power indicate laser heating effect on the GaN nanowires. (intensity in not in scale, full laser power $17 \mathrm{~mW}$, spot size $\sim 10 \times 10 \mu \mathrm{m}^{2}$ ) 

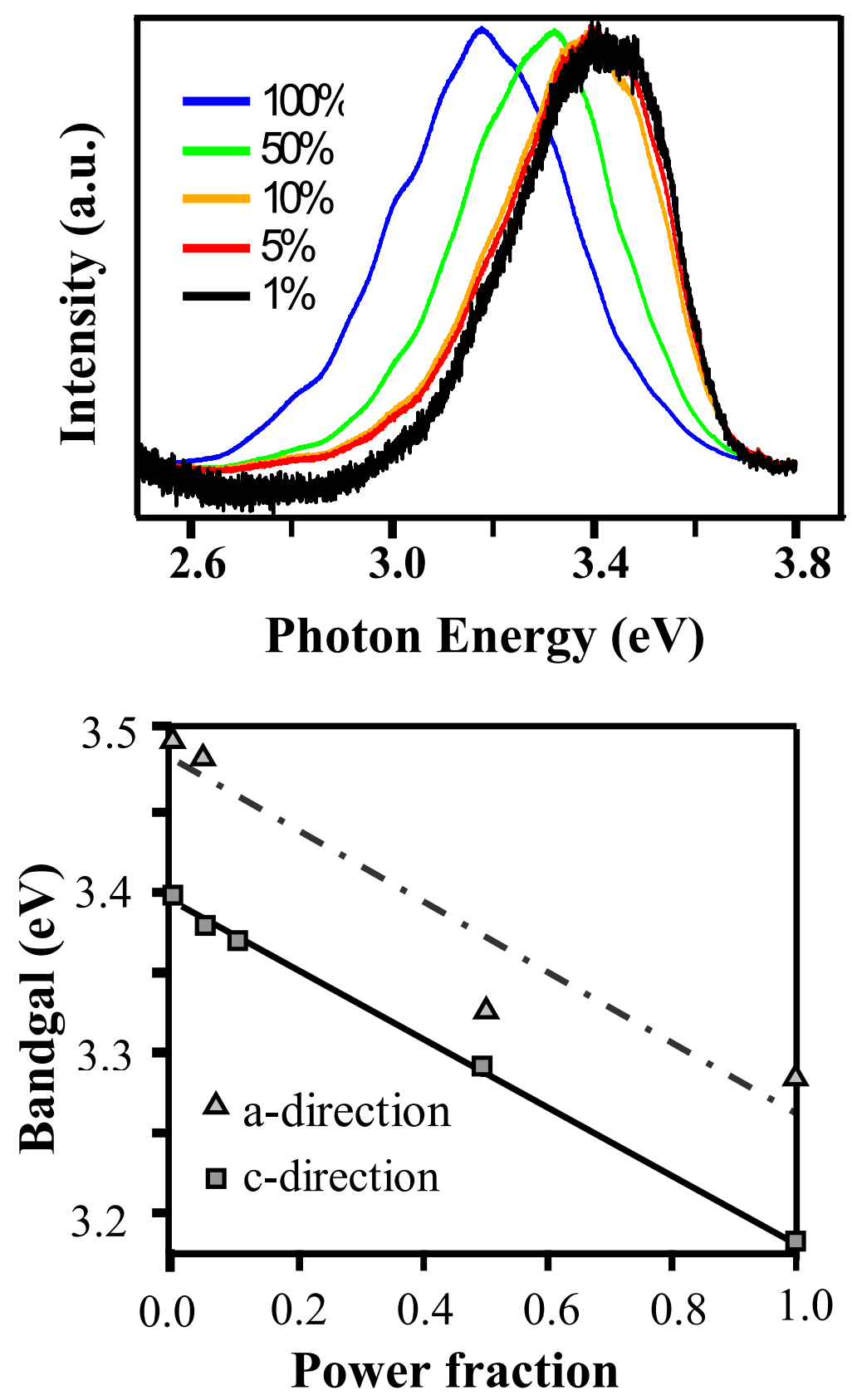

Figure 6.10 (a) UV PL of $<0001>\mathrm{GaN}$ nanowires with different laser power fraction after correction assuming a Gaussian shape for the spectral dip (b) Bandgap of GaN nanowires with $<10-10>$ direction shows blue-shift about $50 \mathrm{meV}$ compared with $<0001>$ GaN nanowires. 


\subsection{Summary}

A simple direct synthesis approach to control the growth directions in $<0001>$ and $<10-10>$ for $\mathrm{GaN}$ nanowires onto amorphous substrates is presented. This is accomplished by controlling the Ga vapor during initial stages of nitridation. The nucleation and growth mechanistic details differ in both cases allowing for growth in different directions: the spontaneous nucleation and basal growth of GaN nanowires from large Ga droplets result in growth in $<0001>$ direction; the Ga droplet-led growth on top of GaN crystals lead to nanowire growth in $<10-10>$ direction. Thus the control of the growth direction is achieved through controlling the Ga flux (the presence of Ga on substrate) in the initial stages. The presence of hydrogen is found to be necessary to help mediate the nanowire growth in both cases. 


\section{CHAPTER 7}

\section{HOMO-EPITAXIAL GROWTH OF GALLIUM NITRIDE NANOWIRES}

\subsection{Introduction}

Defects-free, high quality GaN nanowires have great potential to be a unique unit for nanoelectronic devices fabrication [Huang, Y., et al., 2002]. Ultralong carbon nanotubes up to several $\mathrm{mm}$ have been synthesized for pursuing potential space rope [Huang, S.M., et al., 2003]. Similarly, ultralong single crystal nanowires could be an ideal seed for growing large area single crystals using homo-epitaxy.

Wurtzite GaN has been widely known as an anisotropic crystal which has both polar surfaces such as (0001) and nonpolar surfaces, e.g., $\{10-10\},\{11-20\}$. The polar surface (0001) is either Ga-terminated or N-terminated. These different surfaces have huge effects in how they influence crystal growth [Gibart, P., 2004] as well as devices performance [Waltereit, P., et al., 2000]. Under equilibrium growth conditions, hexagonal platelets and columns bounded by $\{10-10\}$ planes and (0001) planes are common for GaN since these surfaces possess lower surface energy [Hartman, P. \& Bennema, P., 1980]. Most often, the GaN crystal growth occurs under non-equilibrium conditions. So, the growth kinetics may play an important role in determining the resulting crystal morphology, i.e., from hexagonal platelets to 3-D hexagonal crystals terminated with different facets including columnar shapes. The 1-D growth is special and cannot be explained by the differences in growth kinetics in different directions. However, homo-epitaxy onto 1-D structures could reveal insight into growth kinetics in 
different directions. The growth kinetics could possible explain a variety of 1-D morphologies observed with $\mathrm{GaN}$ such as nanobelts [Jian, J. K., et al., 2003] and nanosaws [Bae, S.Y., et al., 2003].

At present, the only way of determining the growth directions of sub-30 nm nanowires which lack any faceting details and appear rounded even in a field emission SEM is TEM analysis. Therefore, there is a need for a simple procedure to determine the growth directions of all nanowires in a sample with high statistical accuracy.

The availability of GaN nanowire samples with two distinct growth directions prompted our studies on homo-epitaxy for various reasons: Firstly, the homo-epitaxy onto these wires allow us to uniquely study the homo-epitaxial growth kinetics on known surface conditions and examine the surface kinetic faceting of the underlying nanowires as they grow thicker in radial direction; Secondly, the homo-epitaxial growth of longer nanowires is hypothesized here to grow larger $\mathrm{GaN}$ crystal, i.e., investigate $\mathrm{GaN}$ nanowire as possible seeds for growing large crystals. Here, a set of homo-epitaxial growth studies were done using pre-synthesized GaN nanowires to study the morphological evolution of 2-D and 3-D morphologies from 1-D structures. The results from this study are directly applicable to synthesis of nanostructures of other III-nitrides and zinc oxide $(\mathrm{ZnO})$ materials systems.

\subsection{Experimental}

The homo-epitaxial experiments were conducted using pre-synthesized polycrystalline $\mathrm{GaN}$ powder as the source of $\mathrm{Ga}$. The source was placed on the $\mathrm{SiC}$ susceptor as shown in Fig. 7.1, and the substrate was placed at a distance of $2 \mathrm{~mm}$ from the Ga source. These experiments were performed at a pressure of 20 Torr with ammonia 
flow rate of $50 \mathrm{sccm}$. The substrate temperature was ramped up to $700{ }^{\circ} \mathrm{C}$ and then the flow of $\mathrm{NH}_{3}$ started while continuing the temperature ramping to $900{ }^{\circ} \mathrm{C}$ in about 10 minutes. The substrates were kept at 20 Torr, $900{ }^{\circ} \mathrm{C}$ for $1-2$ hours. After the nitridation at $900{ }^{\circ} \mathrm{C}$ for $1-2$ hours, the experiment was shutdown by ramping the temperature down to $700{ }^{\circ} \mathrm{C}$ in 10 minutes, followed by replacing the $\mathrm{NH}_{3}$ flow with $\mathrm{N}_{2}$. Finally, the substrate was cooled down further under flowing nitrogen to room temperature. The homo-epitaxial experiments were performed using the same setup and similar conditions as reactive vapor transport except the substrates with pre-synthesized GaN nanowires were used. In all cases, the temperature was measured using a C-type thermocouple which was calibrated earlier using aluminum (melting point of $660{ }^{\circ} \mathrm{C}$ ) and germanium (melting point of $937^{\circ} \mathrm{C}$ ).

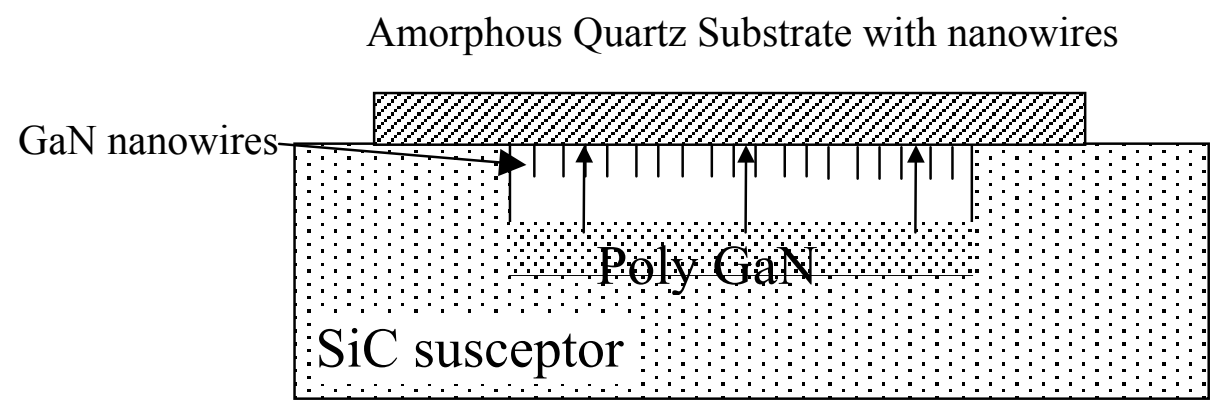

Figure 7.1 Homo-epitaxial experiments onto $\mathrm{GaN}$ nanowire in $\mathrm{NH}_{3}$ atmosphere.

\subsection{Result and Discussion}

\subsubsection{Homo-epitaxial Growth onto $<0001>$ Nanowires}

The morphologies of the homo-epitaxial growth onto the nanowires with $<0001>$ growth direction showed growth of hexagonal prisms at discrete locations of the nanowires but not uniformly over the entire length of the nanowires. (See Fig. 7.2) The 
planes of the prism belong to family $\{10-10\}$ and $\{10-11\}$. In many cases, the hexagonal prismatic growth occurred at ends of the nanowires (Fig. 7.2(b) \& (c)). In some cases, the occurrence of hexagonal prisms happened at both ends of the nanowires (Fig. 7.2(a)). In all cases, the hexagonally shaped morphological growth ended with flat hexagonal facet. The rest of the nanowire did not thicken during the homo-epitaxial growth. The results suggest that the surface transport (diffusion) lengths of adatoms on the $20 \mathrm{~nm} \mathrm{GaN}$ nanowires with $<0001>$ growth direction are quite large in excess of several tens of micrometers. All the species adsorbed onto any part of the nanowires transport to the growing end of the nanowire and get incorporated into the hexagonal prism crystal.
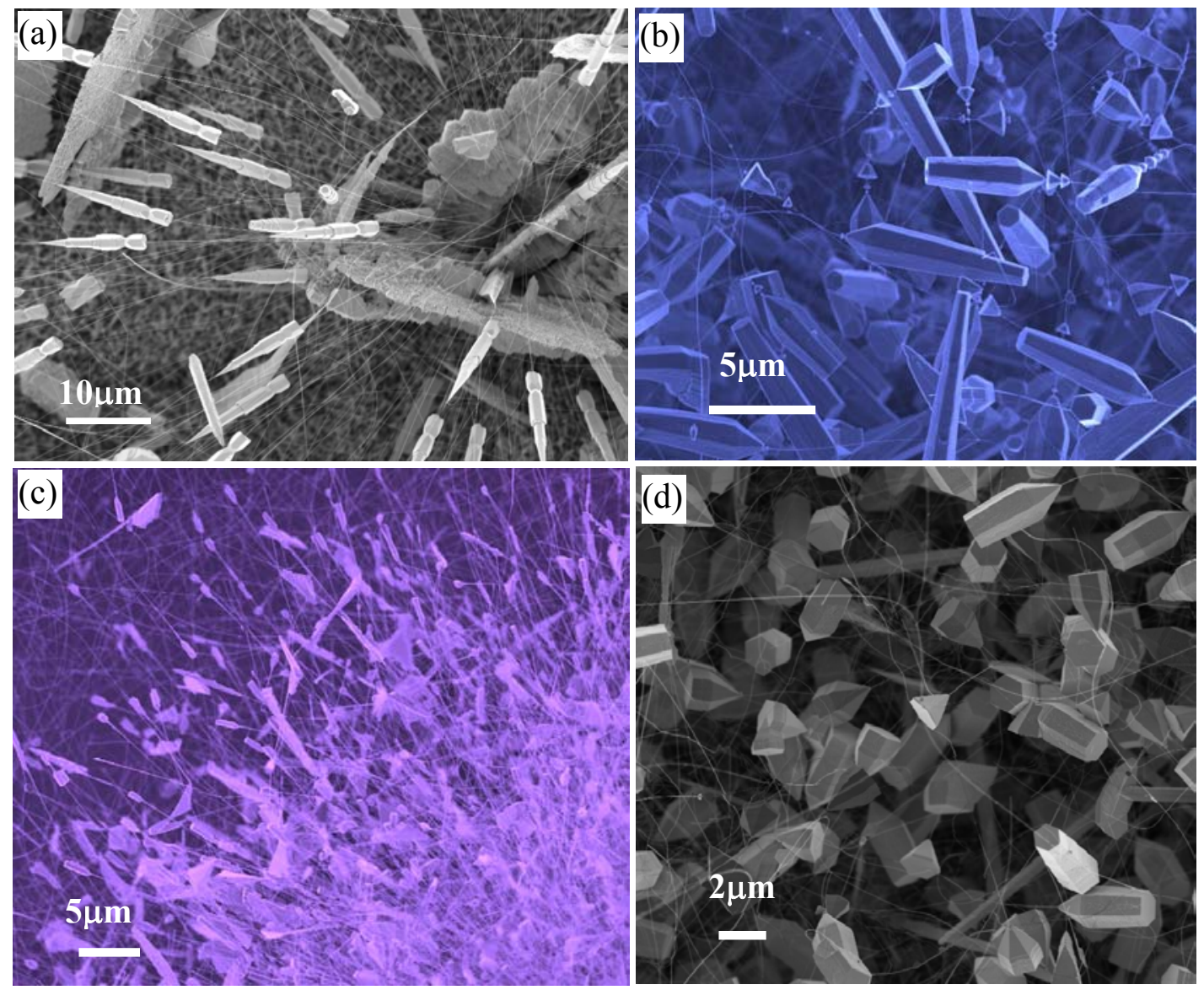

Figure 7.2 (a-d) SEM images showing the resulting morphologies with homo-epitaxial growth onto $<0001>$ GaN nanowires. 
Fig. 7.3(a) shows a low magnification TEM micrograph of the homo-grown GaN hexagonal prism from $<0001>$ direction nanowire. The hexagonal prism is as big as 500 $\mathrm{nm}$ and the wire at the tip is about $40 \mathrm{~nm}$. The growth direction of the wire with prism is $<0001>$ from the high-resolution image in Fig. 7.3(b). The presence of stacking faults on the c-plane is also seen due to stacking error.
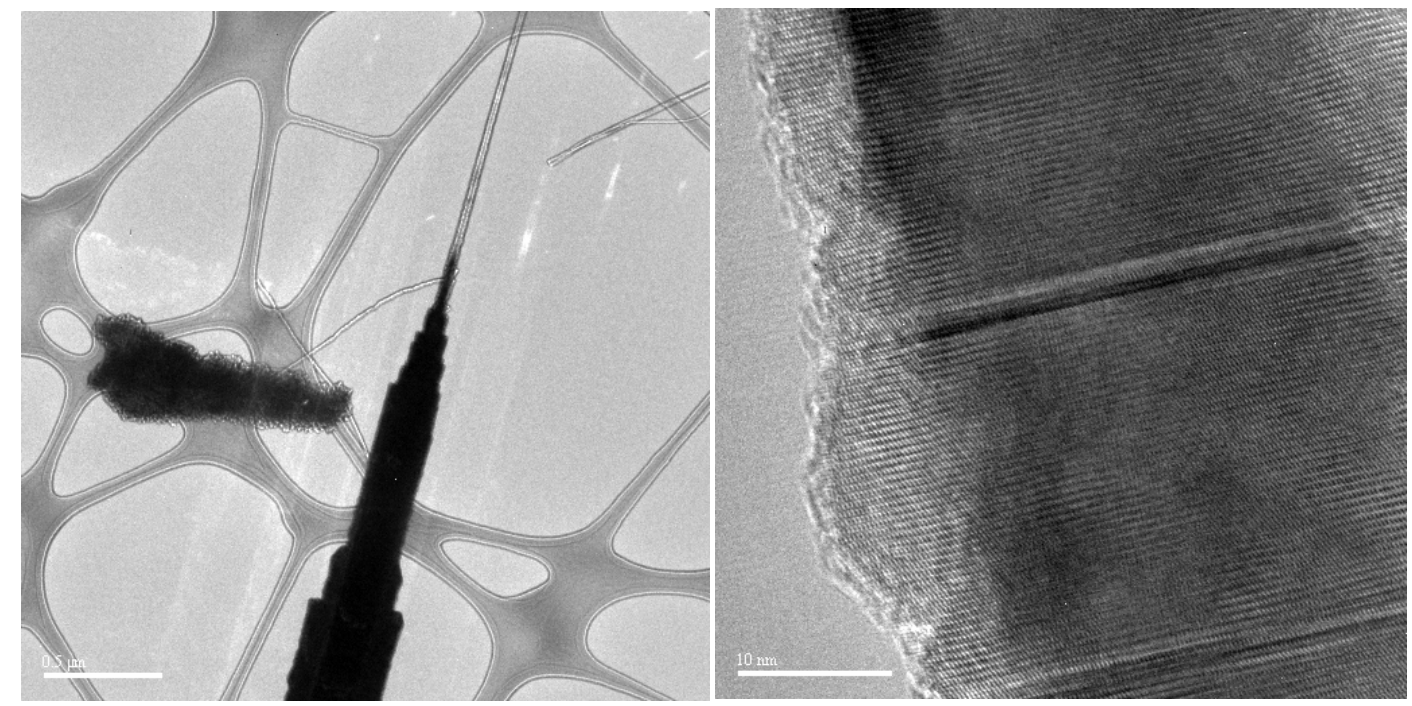

Figure 7.3 (a) Low magnification TEM image of homo-grown GaN hexagonal prisms from $<0001>$ nanowire; (b) HRTEM micrograph of the nanowire showing stacking faults in c-plane.

\subsubsection{Homo-epitaxial Growth onto $<10-10>$ Nanowires}

The homo-epitaxial growth onto GaN nanowires with $<10-10>$ growth direction resulted in belt-shaped morphology as shown in Fig. 7.4(a). A micro-belt which is about $900 \mathrm{~nm}$ wide shown in Fig. 7.4(b) clearly shows growth from one side of the nanowire. Most of the belts grew out of the nanowires all along the length of the nanowires. But there are some belts growing preferentially in an island fashion were also observed, indicating the possibility of enhanced adatom transport on these thin nanowires also. (See Fig. 7.5) A TEM image of $100 \mathrm{~nm}$ wide belt is shown in Fig. 7.6(a). One side of the belt 
is smooth while the other side is rough. The direction perpendicular to the growth direction is determined to be $<0001>$ both from the SAD pattern and the lattice spacing shown in Fig. 7.6(b). The large top surface of these microbelts is (0-110) plane and the Raman spectrum of these microbelts with only $\mathrm{E}_{1}(\mathrm{TO})$ peak showing also support the conclusion. (See the Fig. 7.7) For hexagonal GaN crystals the (0001) surface is polar, which means it has two kinds of surface along c-direction, Ga-terminated surface, (0001) and N-terminated surface, $(000-1)$. It is suggested that the smooth side is Ga-terminated surface and the rough side is $\mathrm{N}$-terminated surface.
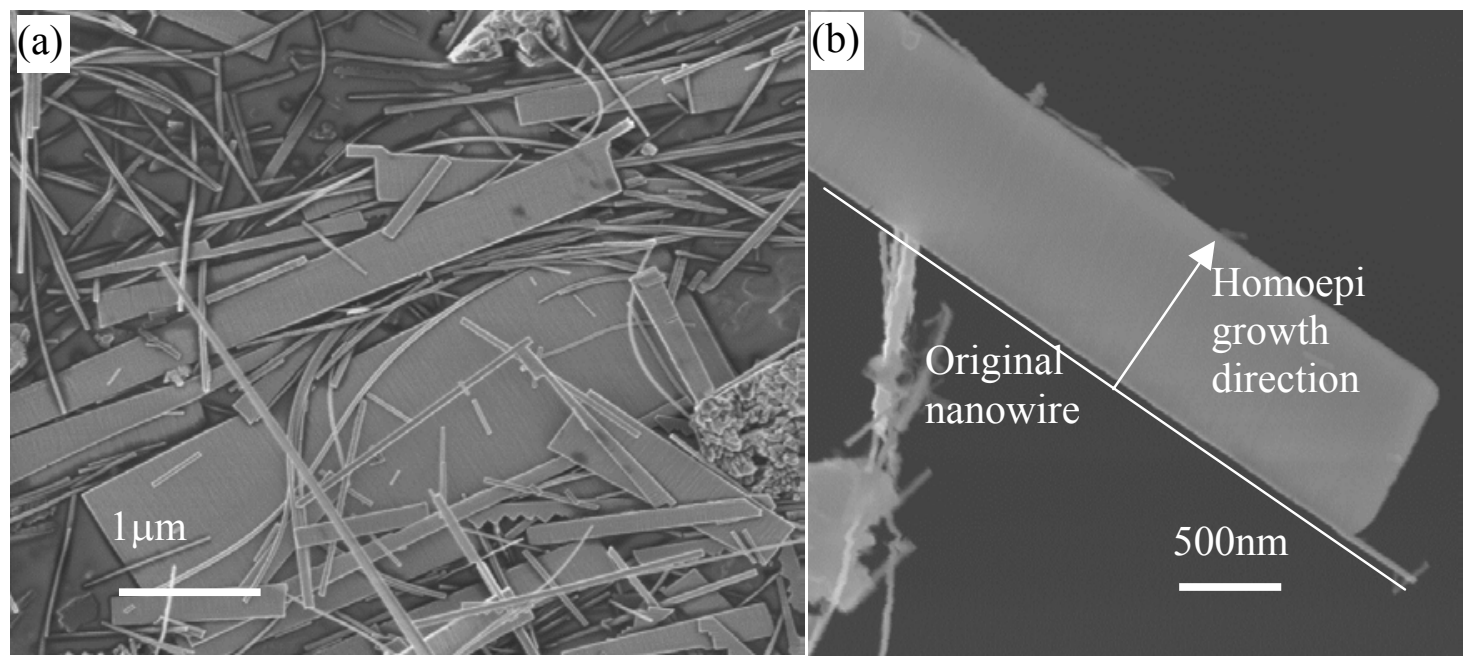

Figure 7.4 (a) SEM images showing the resulting micro-belt morphologies obtained with homo-epitaxial growth onto $<10-10>$ nanowires. (b) A $900 \mathrm{~nm}$ microbelt with preferential growth from one edge of the $<10-10>$ nanowire. 


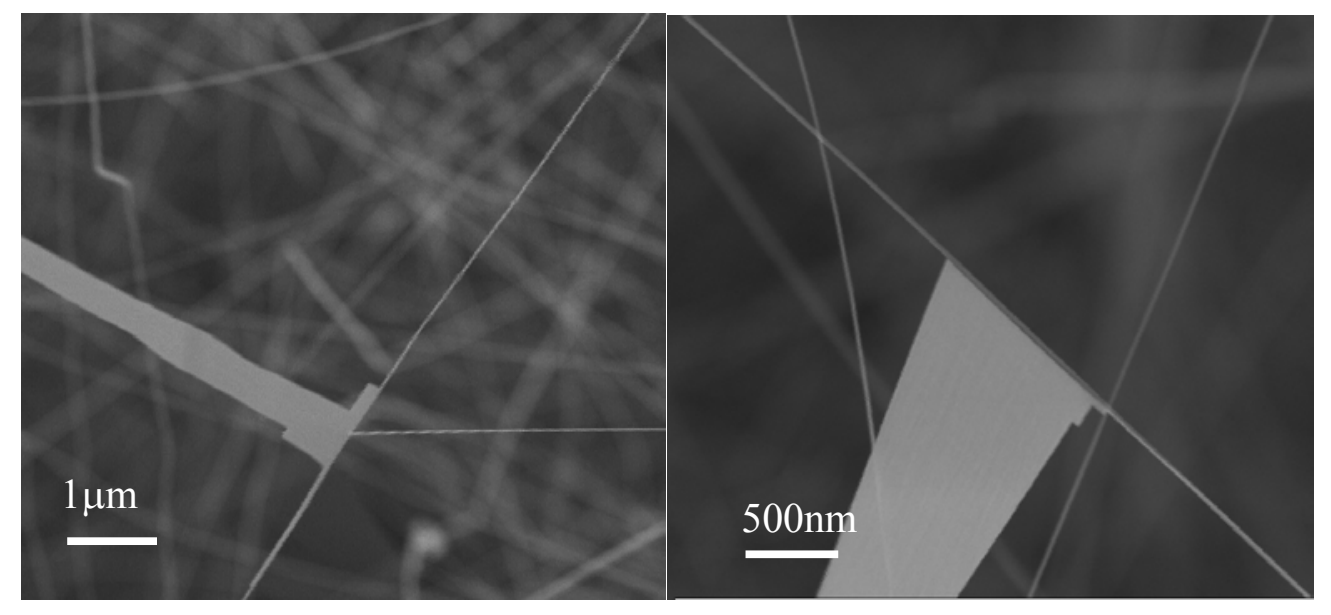

Figure 7.5 Preferential growth of microbelts from $<10-10>$ GaN nanowires with vapor transport growth in presence of $\mathrm{NH}_{3}$ in some cases.
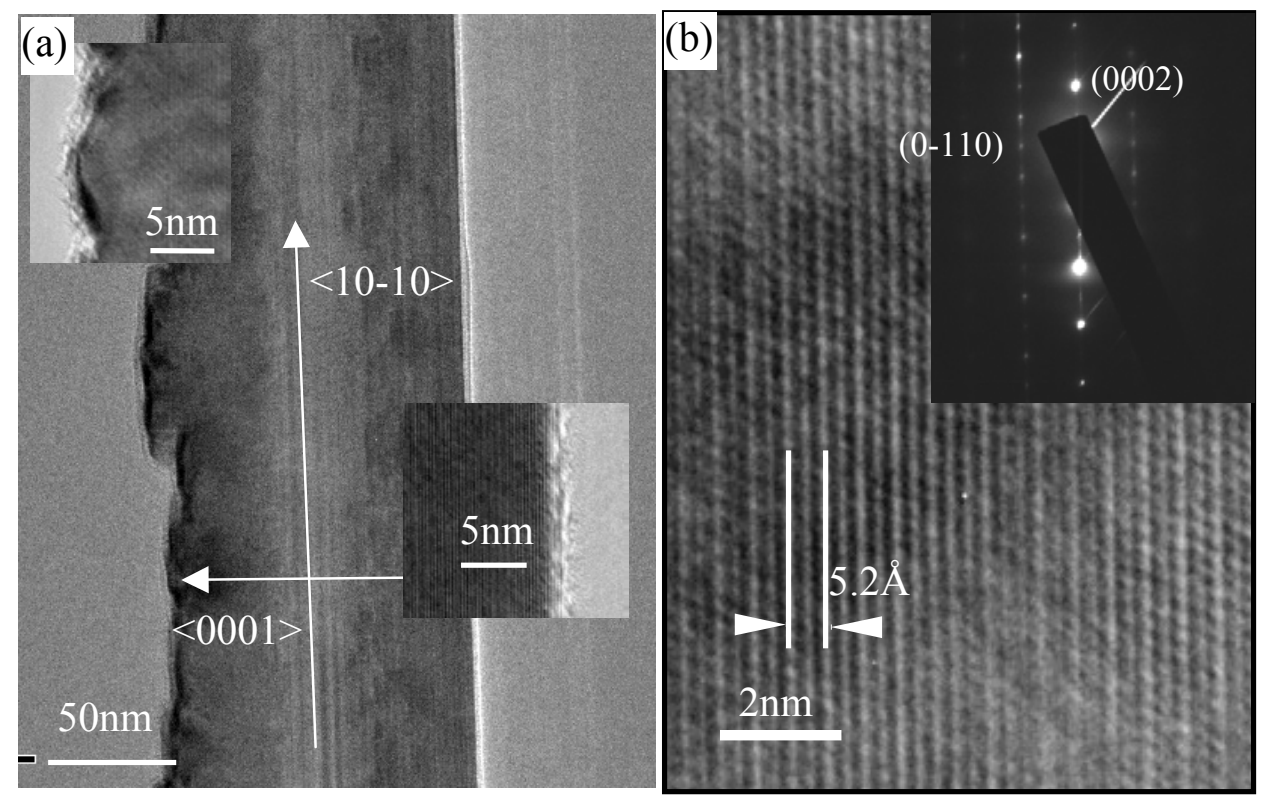

Figure 7.6 (a) TEM micrographs of a homo-epitaxial grown $100 \mathrm{~nm}$ micro belts from $<10-10>\mathrm{GaN}$ nanowire showing Ga terminated and N-terminated surfaces; (b) SAD pattern with zone axis $(\mathrm{ZA})<11-20>$ and high resolution TEM indicating that the direction perpendicular to the nanowire growth is $<0001>$. 


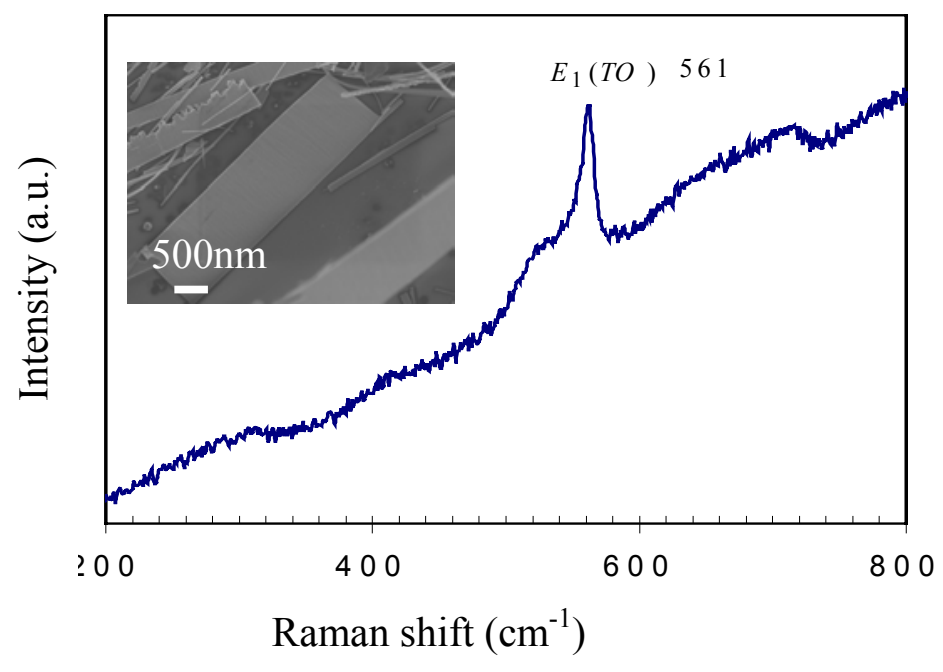

Figure 7.7 Raman spectrum of the GaN microbelts showing only $E_{1}(\mathrm{TO})$ peak at $561 \mathrm{~cm}^{-1}$ indicating the large surface of the microbelts is perpendicular to the c-axis.

\subsubsection{Kinetic Growth of GaN Nanowires}

The mean free path for $\mathrm{Ga}$ vapor, $\mathrm{NH}_{3}$, and atomic $\mathrm{N}$ is about 1-3 $\mu$ m under the growth conditions and therefore the concentrations are not expected to be much different along the length of the nanowires. The surface diffusion length, defined as the distance that adatom adsorbed onto the surfaces travels before it either desorbs or incorporates into the lattice, is given by [Feynman, R. P., et al., 1966]:

$$
\lambda_{S}=\sqrt{D_{S} \tau_{S}}=\sqrt{D_{S, 0} \exp \left(-\frac{E_{S, D}}{k_{B} T}\right) \tau_{S}}
$$

Where $\lambda_{S}$ is the surface diffusion length, $D_{S}$ is the diffusion coefficient, $\tau_{S}$ is the lifetime of the diffusion event, $D_{S, 0}$ is the $T$ independent diffusion coefficient, $E_{S, \mathrm{D}}$ is the diffusion activation energy, and $k_{B}$ is the Boltzman constant. Based on the desorption-limited lifetime, the surface diffusion length of Ga on surface of molten Ga was estimated to be 
on the order of $1 \mathrm{~nm}$ at a temperature of $900^{\circ} \mathrm{C}$ [Koleske, D. D., et al., 2000]. The surface diffusion length of Ga under MOCVD growth conditions was estimated to be on the order of $10-100 \mathrm{~nm}$ at $1050^{\circ} \mathrm{C}$ according the lattice incorporation model [Koleske, D. D., et al., 2000]. Recently, Jensen et al. [Jensen, L.E., Samuelson, L., et al., 2004] reported that the surface diffusion length of In on 40-170 nm InAs nanowires is greater than 10 $\mu \mathrm{m}$, and they also inferred that the diffusion length decreases with increasing wire diameters using experiments with different spacings between nanowires. Most relevantly, other studies have shown that the diffusivity on a liquid surface is much higher that on a solid surface [Binh, V. T., Melinon, P., 1985]. The presence of two atomic layers of In or Ga could enhance the surface transport of adatoms on GaN surfaces [Neugebauer, J., et al., 2003].
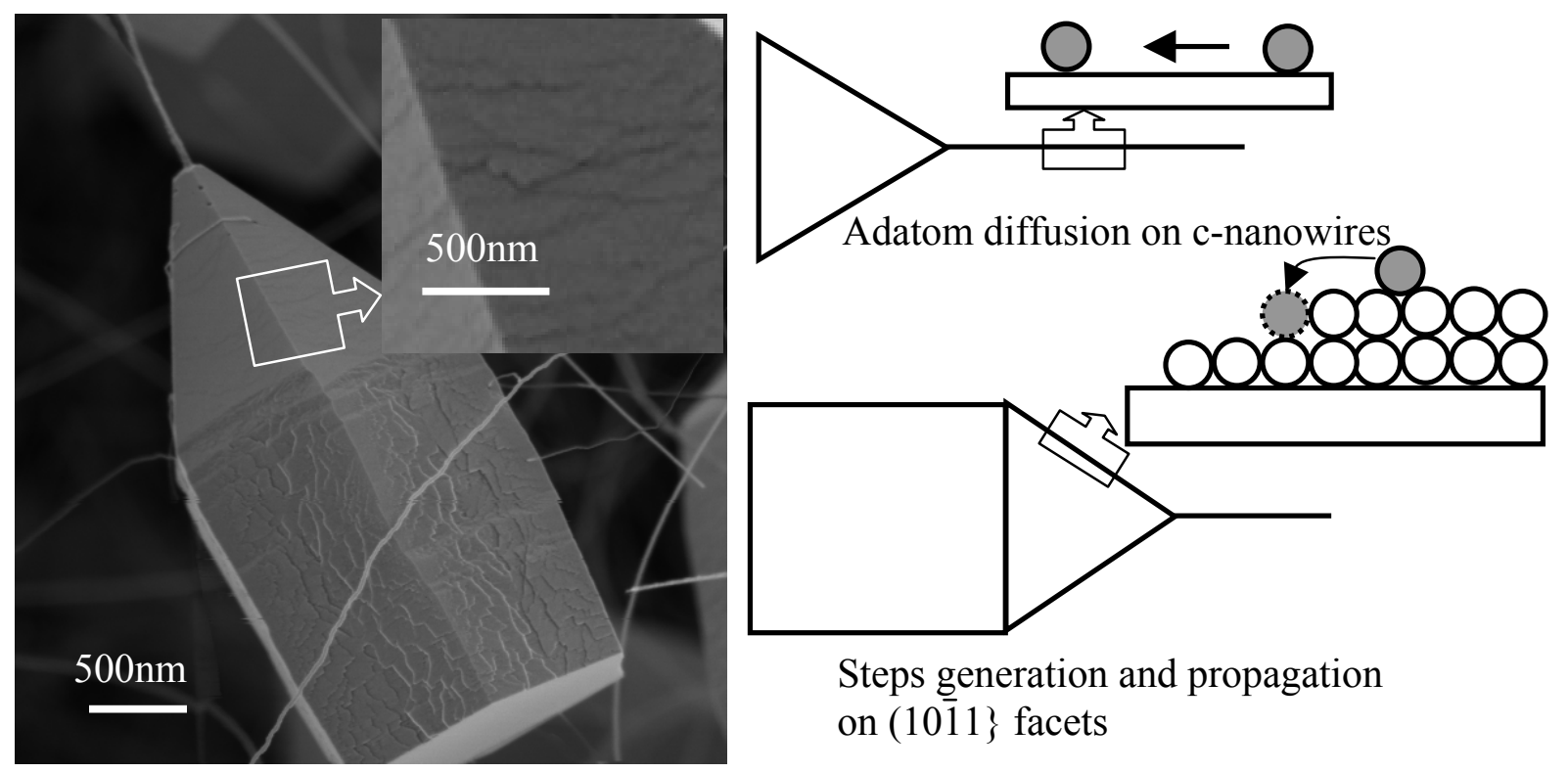

Steps generation and propagation on $(10 \overline{1} 1\}$ facets

Figure 7.8 (a) Growth steps on $\{10-11\}$ pyramidal side facets indicating growth from nanowires onto hexagonal prisms. (b) Schemes of 1-D diffusion growth processes forming hexagonal islands. 
The growth steps on the $\{10-11\}$ surfaces of the hexagonal prism can be clearly seen in Fig. 7.8(a). The steps seem to be generated at the tip of the prisms and propagate toward the hexagonal bottom, which indicates that the adatoms transported from the wires to the hexagonal islands. The schemes of growth steps of homo-epitaxial processes with their corresponding SEM images of nanowires are shown in Fig. 7.9.

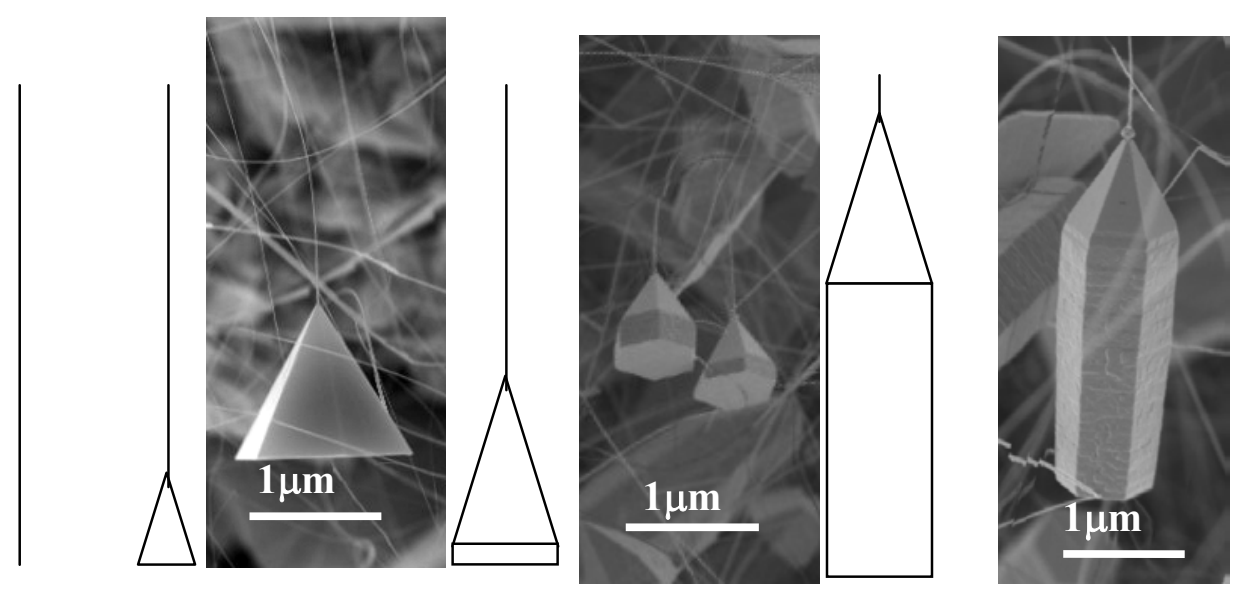

Figure 7.9 Sketch of homo-epitaxial growth processes for homo-epitaxial growth onto $<0001>$ GaN nanowires.

The homo-epitaxy of these GaN nanowires with $<10-10>$ growth direction with polar (0001) surfaces resulted preferential growth primarily on Ga terminated surface, while the growth on other side (nitrogen terminated) is completely absent. Different growth rate depending on polarity of polar crystals surfaces has been found for $\mathrm{GaN}$ [Rouviere, J.L., et al., 1996] as well as ZnS [Moore, D., et al., 2004].

Existence of a singular polar axis [0001] in wurtzite GaN results in spontaneous and piezoelectric polarization along the polar axis which is the crystallographic orientation of most grown GaN [Yu, E.T. \& Manasreh, M.O., 2002]. The presence of the polarization distorts the ideal flat-band profile of MQW structures in III-nitrides based 
devices and therefore reduces the luminous quantum efficiency [Fiorentini, V., et al., 1999]. Optoelectronic devices made out of III-nitrides with $\{10-10\}$ orientation, which is non-polar, exhibited higher efficiency and shows promise for high power LEDs [Waltereit, P., et al., 2000]. The homo-epitaxial growth studies have shown that GaN micro-belts as long as $10 \mu \mathrm{m}$ and several $\mu \mathrm{m}$ wide had been grown from $<10-10>$ nanowires (Fig. 7.10(a)). These results indicate that it is possible to grow large area $\{10$ $10\}$ oriented $\mathrm{GaN}$ single crystal out of long $\mathrm{GaN}<10-10>$ nanowires (Fig. 7.10 (b)).

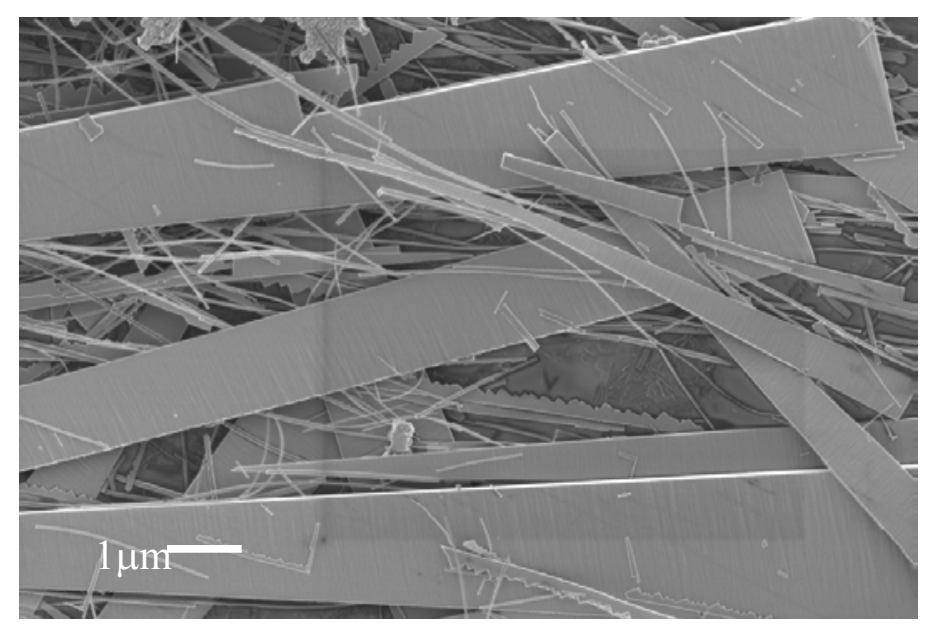

$(10-10)$
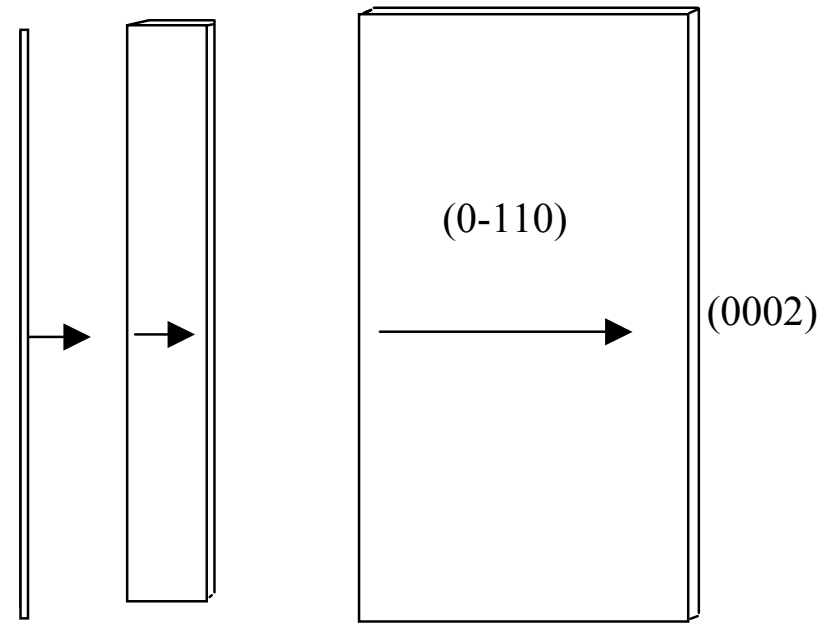

Figure 7.10 (a) $\{10-10\}$ oriented GaN crystal grown from $\mathrm{GaN}<10-10>$ nanowires (b) Schemes of microbelts formation from $<10-10>$ nanowires. 
The above results present a simple way of determining the growth directions of the nanowires. As seen in FESEM images, the $<0001>$ and $<10-10>\mathrm{GaN}$ nanowires with diameters less than $30 \mathrm{~nm}$ all have rod-like shape from SEM observation. But the homo-epitaxial growth onto these two different directional nanowires gives dramatic contrast between the morphologies: the hexagonal shaped micro crystals from $<0001>$ nanowires and flat rectangular shaped micro belts from nanowires with $<10-10>$

direction. The homo-epitaxy of nanowires could also explain the growth mechanism of all ribbon or belt shaped nanostructures. Most of the GaN nanoribbon [Li, Z. J., et al., 2001] or nanobelts [Jian, J. K., et al., 2003] have similar width along the growth direction which excludes the possibility of growth simultaneously both in radial and growth direction since this would always results in tapered width along the growth direction.

\subsection{Summary}

The homo-epitaxial growth onto nanowires with different directions resulted in distinct morphologies. Also, the observed island growth regime on both types of nanowires shows that the surface diffusion length on the surface of these nanowires is enhanced. The homo-epitaxy also proves to be a simple way of identifying the growth direction of sub-30 nm size wires through morphology of resulting structures. 


\section{CHAPTER 8}

\section{CONCLUSIONS}

A novel concept for self-oriented GaN crystal growth using direct nitridation of molten gallium had been demonstrated. The nitridation experiments were performed with Ga films covered on amorphous quartz substrates using ECR-MW generated nitrogen plasma. The main conclusions from the studies are:

- Nitrogen-dissolved molten gallium melts under high temperature wets GaN and results in spreading of gallium melts over the substrates;

- GaN crystals nucleate from nitrogen saturated gallium, the growth and the alignment of these hexagonal platelets crystals by flow of gallium melts produce highly textured GaN films over molten gallium;

- GaN films grown on top of molten gallium are stress free and have low native donor concentration on the order of $10^{17} / \mathrm{cm}^{3}$, while the donor concentration of GaN films grown directly on quartz substrates is over $10^{19} / \mathrm{cm}^{3}$ due to the oxygen diffusion from the quartz $\left(\mathrm{SiO}_{2}\right)$ substrates;

- Self-oriented GaN films as large as $5 \mathrm{~mm} \times 5 \mathrm{~mm}$ size were obtained using a stationary substrate stage. The overall misorientation was less than 2.2 degrees between GaN domains;

- The uncontrolled surface profile (wavy nature) of gallium film during nitridation confines the size of the oriented film to few $\mathrm{mm}$ in dimensions. 
One-dimensional nanostructures of $\mathrm{GaN}$ in different directions have been synthesized by controlling gallium flux. GaN nanowires as thin as $15 \mathrm{~nm}$, up to one hundred microns long with two distinct growth directions resulted from two different approaches. The studies give the following conclusions:

- Presence of atomic hydrogen reduces the wettability of gallium over $\mathrm{GaN}$ and prevents the coalesce of $\mathrm{GaN}$ nuclei;

- $\mathrm{GaN}$ nanowires with $<0001>$ growth direction (c-direction) were grown with spontaneous nucleation and basal growth from Ga melts;

- Reactive vapor transport of Ga in dissociated ammonia results in GaN crystals followed by growth of GaN nanowires through tip-led liquid phase epitaxy. Nanowires grew with $<10-10>$ direction (a-direction) due to preferential wetting of $\mathrm{Ga}$ on $\{10-10\}$ surfaces of $\mathrm{GaN}$.

Homo-epitaxial studies onto these two distinct directional GaN nanowires reveals clear differences due to kinetic growth processes. Homo-epitaxial growth onto $<10-10>$ $\mathrm{GaN}$ nanowires has shown to be a promising approach for synthesizing large area $\mathrm{GaN}$ crystals. The conclusions from these studies are:

- Homo-epitaxial experiments onto c-direction and a-direction GaN nanowires produced distinct growth morphologies, e.g., hexagonal prisms for cdirectional wires and micro-belts for a-directional nanowires;

- Kinetic growth processes of nanowires exhibited a new phenomenon of enhanced surface transport of adatoms on nanowires up to tens of microns, especially on $<0001>$ nanowires; 
- Homo-epitaxial experiments onto $<10-10>$ nanowires conclusively reveals the nucleation and growth mechanisms responsible for nano-belts or nano-ribbon type 1-D structures;

- The homo-epitaxial studies present a simple way of determining the growth directions of less than $30 \mathrm{~nm}$ nanowires for GaN over large quantity. 


\section{CHAPTER 9}

\section{PERSPECTIVES}

This dissertation explored new concepts of synthesizing large area gallium nitride bulk crystals. Nucleation and growth of GaN crystals from molten Ga produced large area, highly c-plane textured GaN films by utilizing the self-spreading phenomenon of nitrogen saturated gallium melts. Direct synthesis of GaN nanowires with control on two different growth directions without single crystal templates has also been presented. Homo-epitaxial growth onto nanowires also shows the possibility of producing large area, low defects single crystals. Based on the obtained results, the following studies could be further explored to advance these novel techniques.

\subsection{Large Area GaN Crystal Growth Using Rotating Substrate Stage}

The preliminary results had demonstrated the concept of self-oriented gallium nitride crystals growth on top of molten gallium under stationary substrate stage. The area of the oriented films is still small compared with the area of the overall substrates. One possible reason behind this is the non-uniformity of gallium film thickness and its temperature. The misorientation between individual grains is still a little larger due to uncontrolled flow pattern of the molten gallium. Therefore better control on the flow pattern of Ga and more uniform temperature distribution are necessary for improving the area and quality of self-oriented GaN crystal growth.

A customer-built rotating substrate stage for the ECR plasma (1-100 mTorr) reactor is shown in Fig. 9.1. The 4-inch diameter rotating substrate stage is radiatively 
heated by a tungsten foil heater. The capabilities of substrate stage include controllable rotation speeds in excess of $2000 \mathrm{rpm}$ at a temperature of $800-1050{ }^{\circ} \mathrm{C}$, uniform heating over the entire 2-inch substrate, easy wafer mounting, and replaceable heating elements.
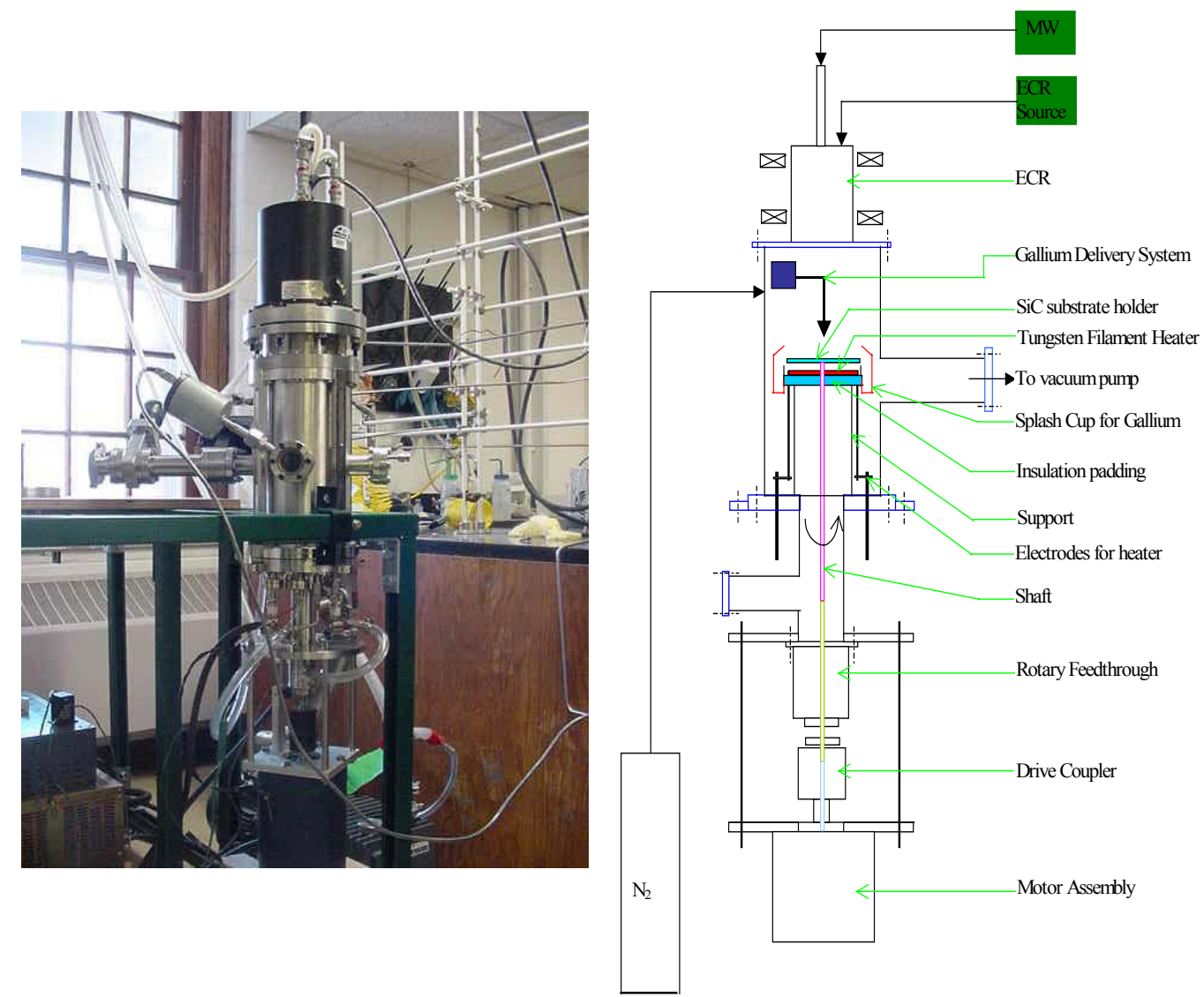

Figure 9.1 ECR-MW reactor with rotating substrates stage for controlling the flow of molten gallium for large area oriented $\mathrm{GaN}$ synthesis.

\subsection{Electronic Properties of GaN Nanowires}

Wurtzite $\mathrm{GaN}$ has been widely known as an anisotropic material. The band gap value [Kuykendall, T., et al., 2004], thermal properties [Levinshtein, et al., 2001] as well as electronic properties [Feng, D. P., et al., 1999] have been found to be dependent on crystallographic orientations. Having obtained GaN nanowires with two distinct growth 
directions, e.g., $<0001>$ and $<10-10>$, it prompts us to study their orientation dependent electronic properties such as electron mobility.

A field effect transistor (FET) shown in Fig. 9.2 (a) could be made out of a nanowire to measure the electronic properties. The modulation of the channel conductance through gate effect could tell the nanowires are either n-type or p-type doped, for example, if the conductance increases with the increasing positive gate voltage $\mathrm{V}_{\mathrm{g}}$, the materials is n-type doped. The carrier concentration (electron) can be estimated from:

$$
\begin{aligned}
& n_{e}=\frac{Q}{e \pi r^{2} L} \\
& Q=C V_{t h}=\frac{2 \pi \varepsilon \varepsilon_{0} L V_{t h}}{\ln \left(\frac{2 h}{r}\right)}
\end{aligned}
$$

where $\mathrm{n}_{\mathrm{e}}$ is the electron density, $\mathrm{cm}^{-3}$;

Q is total charge, Coulomb (C);

$\mathrm{C}$ is the nanowire capacitance, Farad, $1 \mathrm{~F}=9.65 \times 10^{4} \mathrm{C}$;

e is the charge of one electron, $1.60 \times 10^{-19} \mathrm{C}$;

$r$ is the radius of the nanowire and $\mathrm{L}$ is the length of the nanowire;

$\varepsilon$ is the dielectric constant of the oxide, for $\mathrm{SiO}_{2}, \varepsilon=3.9$;

$\varepsilon_{0}$ is the permittivity of the free space, $8.85 \times 10^{-14} \mathrm{~F} / \mathrm{cm}$;

$\mathrm{V}_{\text {th }}$ is the threshold voltage, $\mathrm{V}$;

$\mathrm{h}$ is the thickness of the oxide, $\mathrm{cm}$;

The carrier mobility $\mu$ can be determined from the transconductance of the FET using the following equation: 


$$
\frac{d I_{s d}}{d V_{g}}=\mu \frac{C}{L^{2}} V_{s d}
$$

where $I_{s d}$ is the current between the source and drain, A;

$\mathrm{V}_{\mathrm{g}}$ is the gate voltage, $\mathrm{V}$;

$\mu$ is the carrier mobility, $\mathrm{cm}^{2} / \mathrm{V} \cdot \mathrm{s}$;

$\mathrm{V}_{\mathrm{sd}}$ is the voltage between the source and drain, $\mathrm{V}$;

This work is being carried through collaboration with Prof. Alphenaar in

Electrical and Computer Engineering Department. The FET made out of GaN nanowires with $<0001>$ growth direction is shown in Fig. 9.2 (b).
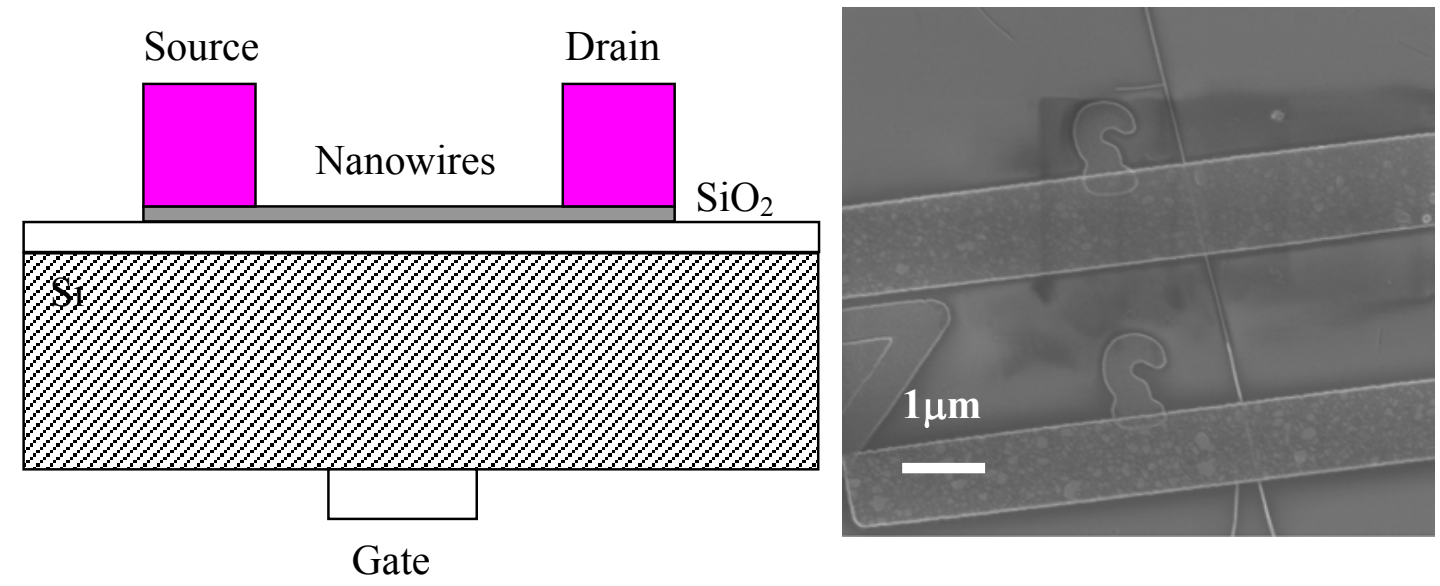

Figure 9.2 (a) Schematic of a nanowire FET and (b) FE-SEM image of FET made out of GaN nanowires for electronic properties measurement.

\subsection{III-nitrides Nanoscale Heterostructures}

Composition modulated semiconductor heterostructures have been widely used in various electronic devices such HBT, HEMT, LED, etc. Direct formation of heterostructures within nanowires provides passivated surfaces for reproducible device behavior at junctions and protective/dielectric/barrier coatings on the nano-scale electronic components for diverse functions. The further development of nanowires with 
built-in heterostructures not only opens up new industrial pathways for device design and construction but these one-dimensional structures will also provide a fertile test bed to evaluate such fundamental physical phenomena as interface electrodynamics and the nature of structural defects which are thought to play a role in charge transfer.

Shown in Fig. 9.3(a) is the SEM image of evaporation of Indium onto GaN nanowires. Indium islands with size of $60-160 \mathrm{~nm}$ formed onto these $30 \mathrm{~nm} \mathrm{GaN}$ nanowires. Nitridation of the indium with nitrogen plasma would form epitaxial InN onto GaN nanowires and then a GaN-core-InN-shell structure would be good candidate to study the electron transport properties.

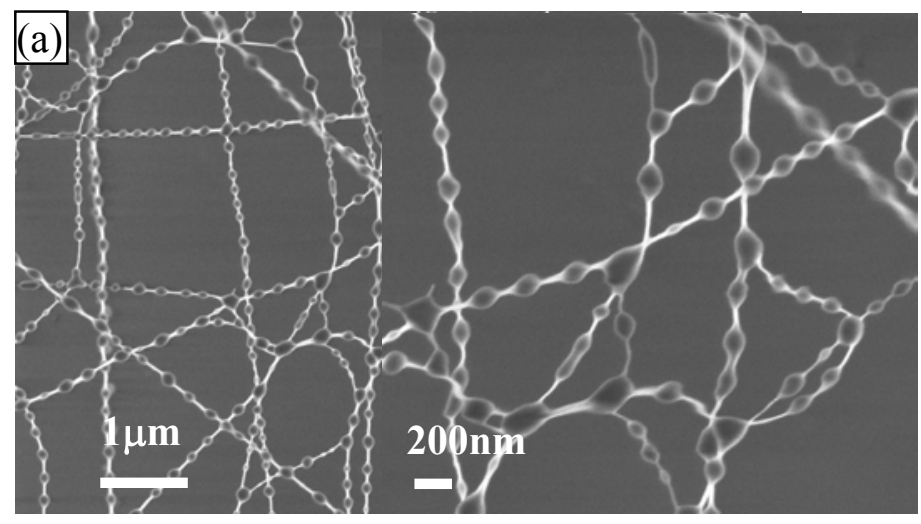
(b)
GaN nanowires

In island

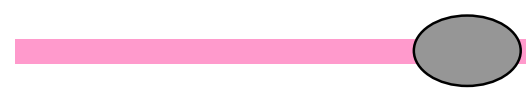

GaN core-InN shell nanostructure

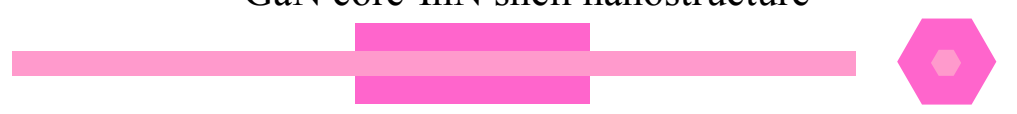

Figure 9.3 (a) Evaporated In islands onto GaN nanowires; (b) GaN-InN core-shell heterostructures. 

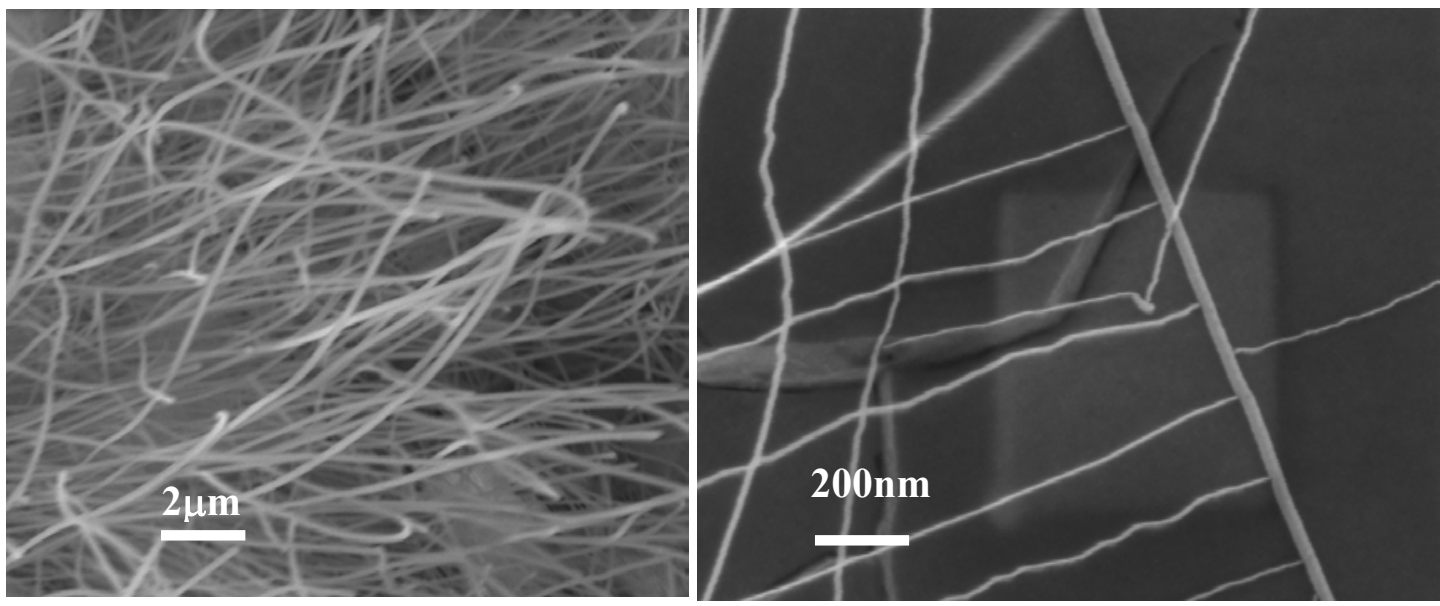

Figure 9.4 (a) GaN core-AIN sheath nanostructure; (b) GaN branched nanostructure.

Shown in Fig. 9.4 (a) are the preliminary results of GaN core -AlN sheath structure through two step processes. First, GaN nanowires was synthesized and then Al metal was evaporated onto the nanowires. The second nitridation converted the Al layer into AlN shell. Fig. 9.4(b) is the branched GaN nanostructures which has potential as sensing network or unit for integrated circuits.
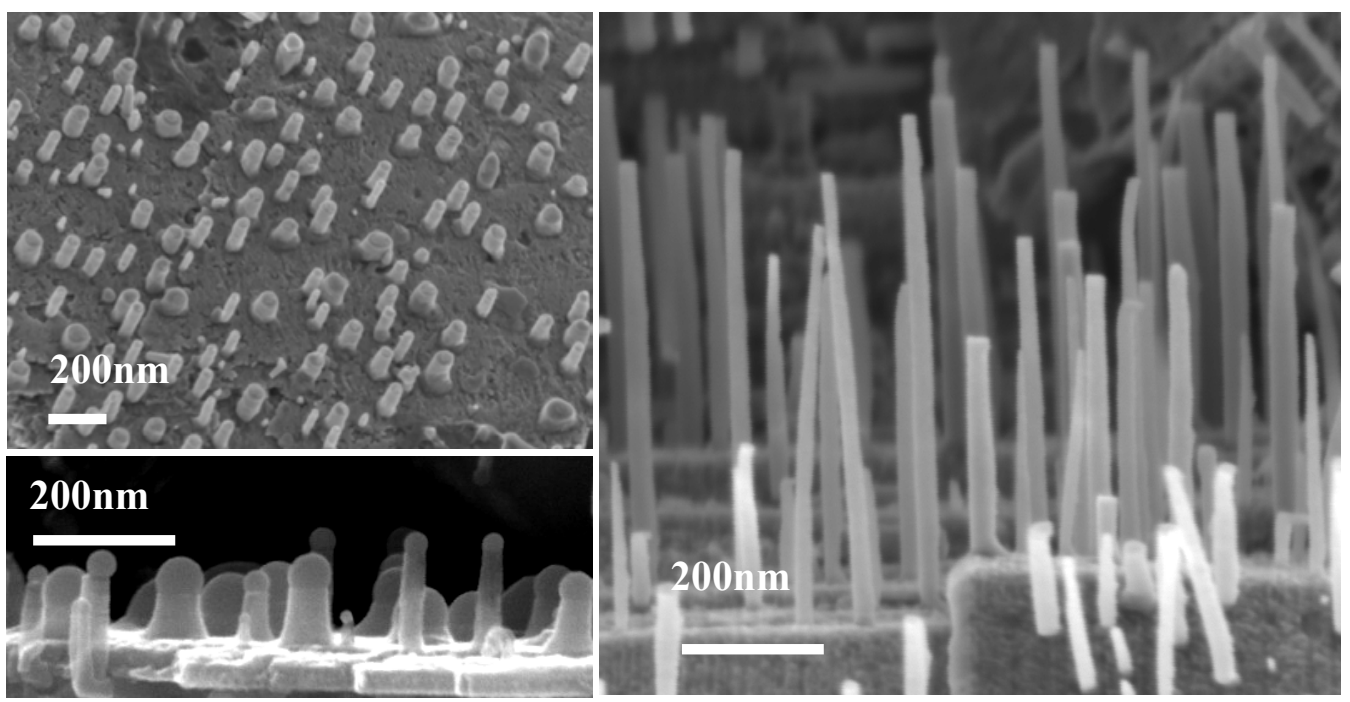

Figure 9.5 Aligned III-nitride nanostructures

Fig. 9.5 shows the aligned III-nitride nanostructures using vapor transport of AlGa-Sn alloy in $\mathrm{NH}_{3}$ atmosphere. A typical vapor-liquid-solid (VLS) growth process is 
clearly seen in these nanostructures. The aligned nanowires are very attractive for practical integration with current IC technology and devices fabrication. 


\section{REFERENCES}

Abernathy, C.R. (1995). Compound semiconductor growth by metallorganic molecular beam epitaxy (MOMBE). Materials Science and Engineering $R$ 14(5), 203-253.

Ahn, S.H., Hong, M.H., et al. (2002). Catalytic growth of high quality GaN microcrystals. Journal of Crystal Growth 234(1), 70-76.

Akasaka, T., et al. (1998). Selective MOVPE of $\mathrm{GaN}$ and $\mathrm{Al}_{x} \mathrm{Ga}_{1-x} \mathrm{~N}$ with smooth vertical facets. Journal of Crystal Growth 189-190, 72-77.

Amano, H., et al. (1986). Metalorganic vapor phase epitaxial growth of a high quality GaN film using an AlN buffer layer. Applied Physics Letters 48(5), 353-355.

Amano, H., et al. (1989). P-type conduction in Mg-doped GaN treated with low-energy electron beam irradiation (LEEBI). Japanese Journal of Applied Physics 28(12), L2112-2114.

Aoki, M., et al. (2000). Growth of GaN single crystals from a Na-Ga melt at $750^{\circ} \mathrm{C}$ and 5 $\mathrm{MPa}$ of $\mathrm{N}_{2}$. Journal of Crystal Growth 218(1), 7-12.

Argoitia, A., Angus, J.C., et al. (1997). Low pressure synthesis of bulk, polycrystalline gallium nitride. Applied Physics Letters 70(2), 179-181.

Asbeck, P.M., Redwing, J., et al. (1997). Piezoelectric charge densities in AlGaN/GaN HFETs. Electronics Letters 33(14), 1230-1231.

Bae, S.Y., et al. (2003). Large-scale synthesis of gallium nitride nanosaws using a chemical vapor deposition method. Chemical Physics Letters 373(5-6), 620-625.

Balka, C.M., et al. (2000). Growth and characterization of GaN single crystals, Journal of Crystal Growth 208(1-4), 100-106.

Baranov, P.G., et al. (1998). Current status of GaN crystal growth by sublimation sandwich technique. MRS Internet Journal of Nitride Semiconductor Research 3, 50.

Basak, B., Lachab, M., Nakanishi, T., Sakai, S. (1999). Effect of reactive ion etching on the yellow luminescence of GaN. Applied Physics Letters 75(23), 3710-3712.

Beaumont, B., Haffouz, S. \& Gibart, P. (1998). Magnesium induced changes in the selective growth of GaN by metalorganic vapor phase epitaxy. Applied Physics Letters 72(8), 921-923. 
Binh, V.T. \& Melinon, P. (1985). On viscous mechanism for surface diffusion at high temperatures $(\mathrm{T} / \mathrm{Tm}>0.75)$ due to formation of a $2 \mathrm{D}$ dense fluid on metallic surfaces. Surface Science 161(1), 234-244.

Boris V. L'vov (2000). Kinetics and mechanism of thermal decomposition of GaN. Thermochimica Acta 360(1), 85-91.

Brown, P.J. \& Forsyth, J.B. (1973). The Crystal Structure of Solids. Edward Arnold, London.

Bykhovski, A., Gelmont, B. \& Shur, M. (1993). The influence of the strain-induced electric field on the charge distribution in GaN-AlN-GaN structure. Journal of Applied Physics 74(11), 6734-6739.

Cadoret, R. \& Trassoudaine, A. (2001). Growth of gallium nitride by HVPE. Journal of Physics-Condensed Matter13(32), 6893-6905.

Cadoret, R. (1999). Growth mechanisms of ( $\left.\begin{array}{lll}0 & 0 & 1\end{array}\right)$ GaN substrates in the hydride vapourphase method: Surface diffusion, spiral growth, $\mathrm{H}_{2}$ and $\mathrm{GaCl}_{3}$ mechanisms. Journal of Crystal Growth 205(1-2), 123-135.

Callahan, M., et al. (1999). Synthesis and growth of gallium nitride by the chemical vapor reaction process (CVRP). MRS Internet Journal of Nitride Semiconductor Research 4, No.10.

Cao, X.A., Pearton, S.J. \& Ren, F. (2000). Advanced processing of GaN for electronic devices. Critical Reviews in Solid State and Materials Sciences 25(4), 279-390.

Cazzanelli, M., et al. (1998). Photoluminescence of localized excitons in pulsed-laserdeposited GaN. Applied Physics Letters 73(23), 3390-3392.

Chandrasekaran, H., \& Sunkara, M.K (2002). Growth of gallium nitride textured films and nanowires on polycrystalline substrates at sub-atmospheric pressures. $M R S$ Symposium Proceeding 693, 159-164.

Chandrasekaran, H. (2002). Substrate independent oriented crystal growth of gallium nitride. M.S. thesis, University of Louisville.

Chen, C.C. \& Yeh, C.C. (2000). Large-scale catalytic synthesis of crystalline gallium nitride nanowires. Advanced Materials 12(10), 738-741.

Chen, Z., et al. (2000). Microstructure and optical properties of $\mathrm{GaN}$ films on sapphire substrates. Materials and Design 21(6), 579-582.

Cherns, D., Henley, S.J. \& Ponce, F.A. (2001). Edge and screw dislocations as nonradiative centers in InGaN/GaN quantum well luminescence. Applied Physics Letters 78(18), 2691-2693. 
Colwell, P.J. \& Klein, M.V. (1972). Raman scattering from electronic excitations in $n$ type silicon carbide. Physical Review B 6(2), 498-515.

Coudurier, L., Eustathopoulos, N., Desre, P., Passerone, A. (1978). Atomic roughness and chemical adsorption in solid-liquid interfaces of binary metallic systems. Acta Metallurgica 26(3), 465-475.

Cumberland, R.W., Blair, R.G., Wallace, C.H., Reynolds, T.K., Kaner, R.B. (2001). Thermal control of metathesis reactions producing GaN and InN. Journal Of Physical Chemistry B 105(47), 11922-11927.

Dassonneville, S., et al. (2001). Luminescence of epitaxial GaN laterally overgrown on (0001) sapphire substrate: Spectroscopic characterization and dislocation contrasts. Journal of Applied Physics 89(7), 3736-3743.

Daudin, B., Rouviere, J.L. \& Arlery, M. (1996). Polarity determination of GaN films by ion channeling and convergent beam electron diffraction. Applied Physics Letters 69(17), 2480-2482

Davis, R. F., et al. (1997). Gas-source molecular beam epitaxy of III-V nitrides. Journal of Crystal Growth 178(1-2), 87-101.

Demangeot, F., et al. (1997). Interplay of electrons and phonons in heavily doped GaN epilayers. Journal of Applied Physics 82(3), 1305-1309.

Derenzo, S.E., Weber, M.J., Moses, W.W., Dujardin, C. (2002a). Measurements of the intrinsic rise times of common inorganic scintillators. IEEE Transactions on Nuclear Science 47(3), 860-864.

Derenzo, S.E., Weber, M.J. \& Klintenberg, M.K. (2002b). Temperature dependence of the fast, near-band-edge scintillation from $\mathrm{CuI}, \mathrm{HgI} 2, \mathrm{PbI} 2, \mathrm{ZnO}: \mathrm{Ga}$ and $\mathrm{CdS}$ :In. Nuclear Instruments and Methods in Physics Research A 486(1-2), 214-219.

Derenzo, S.E., Weber, M.J. Bourret-Courchesne, E. Klintenberg, M.K. (2003). The quest for the ideal inorganic scintillator. Nuclear Instruments and Methods in Physics Research A 505(1-2), 111-117.

Doverspike, K. \& Pankove J.I. (1998). Doping in the III-nitrides, in Gallium Nitride (GaN) I, Academic Press, Eds. J. Pankove \& T. Moustakas, in the Semiconductors and Semimetals Series 50, 259-277. Series Eds. R.K. Willardson \& E.R. Weber.

Ejder, E. (1974). Growth and morphology of GaN. Journal of Crystal Growth 22(1), 4446.

Elwell, D., et al. (1984). Crystal growth of $\mathrm{GaN}$ by the reaction between gallium and ammonia. Journal of Crystal Growth 66(1), 45-54.

Feng, D.P., Zhao, Y. \& Zhang, G.Y. (1999). Anisotropy in electron mobility and 
microstructure of GaN grown by metalorganic vapor phase epitaxy. Physica Status Solidi A 176(2), 1003-1008.

Feynman, R.P., Leighton, R.B., \& Sands, M. (1966). The Feynman Lectures on Physics, Vol. 1, Addison-Wesley, Reading, MA, 41-49.

Florescu, D.I., et al. (2000). Thermal conductivity of fully and partially coalesced lateral epitaxial overgrown GaN/sapphire (0001) by scanning thermal microscopy. Applied Physics Letters 77(10), 1464-1466.

Freitas, J.A., et al. (1998). Optical characterization of lateral epitaxial overgrown GaN layers. Applied Physics Letters 72(23), 2990-2992.

Gemperle, A. \& Gemperlová, J. (1995). Practical accuracy of grain misorientation measurements by Kikuchi line technique. Ultramicroscopy 60(2), 207-218.

Gibart, P. (2004). Metal organic vapour phase epitaxy of $\mathrm{GaN}$ and lateral overgrowth. Reports on Progress in Physics 67(5), 667-715.

Glaser, E. R., et al. (1995). Optically detected magnetic resonance of GaN films grown by organometallic chemical-vapor deposition. Physical Review B 51(19), 1332613336.

Greiner, E. S., Gutowski, J. A. \& Ellis, W. C. (1961). Preparation of silicon ribbons. Journal of Applied Physics 32(11), 2489-2490.

Goldstein, J. I., et al. (1992) Scanning Electron Microscopy and X-Ray Microanalysis. 2nd ed., Plenum Press.

Grossner, M.T. (1999). Low pressure growth of bulk, polycrystalline gallium nitride from gallium/indium alloys. M.S. Thesis, Case Western Reserve University.

Harima, H. (2002). Characterization of GaN and related nitrides by Raman scattering, in III- Nitride Semiconductors Optical Properties I, edited by M .O. Manasreh \& H. X. Jiang, Taylor \& Francis Books, Inc, NY, London.

Harima, H., Sakashita, H., Inoue, T., Nakashima, S. (1998). Electronic properties in doped GaN studied by Raman scattering. Journal of Crystal Growth 189-190, 672676.

Hartman, P. \& Bennema, P. (1980). The attachment energy as a habit controlling factor .1. Theoretical considerations. Journal of Crystal Growth 49(1), 145-156.

Haus, E., Mishra, U.K., Speck, J.S., et al. (2002). The Role of growth conditions on the P-doping of GaN by plasma-assisted molecular beam epitaxy. Journal of Crystal Growth 246(1-2), 55-63.

He, M., Mohammad, S.N., et al. (2001). Growth of GaN nanowires by direct reaction of 
Ga with $\mathrm{NH}_{3}$. Journal of Crystal Growth 231(3), 357-365.

Held, R., et al. (1998). N-limited versus Ga-limited growth on GaN(0001) by MBE using $\mathrm{NH}_{3}$. Surface Review and Letters 5(3-4), 913-934.

Held, R., et al. (1999). Structure and composition of GaN(0001) A and B surfaces. Journal of Applied Physics 85(11), 7697-7704.

Hellman, E.S. (1998). The polarity of GaN: A critical review. MRS Internet Journal of Nitride Semiconductor Research 3, No.11.

Herring, C. (1951). Some theorems on the free energies of crystal surfaces. Physical Review 82, 87-93.

Heying, B., Speck, J.S., et al. (1996). Role of threading dislocation structure on the x-ray diffraction peak widths in epitaxial GaN films. Applied Physics Letters 68(5), 643645 .

Heying, B., Speck, J.S., et al. (2000). Control of GaN surface morphologies using plasma-assisted molecular beam epitaxy. Journal of Applied Physics 88(4), 18551860.

Hirako, A., et al. (2002). GaN-MOVPE growth and its microscopic chemistry of gaseous phase by computational thermodynamic analysis. Journal of Crystal Growth 237239(2), 931-935.

Hiramatsu, K., et al. (2000). Fabrication and characterization of low defect density GaN using facet-controlled epitaxial lateral overgrowth (FACELO). Journal of Crystal Growth 221(1-4), 316-326.

Hong, S.K., et al. (2000). Origin of hexagonal-shaped etch pits formed in (0001) GaN films. Applied Physics Letters 77(1), 82-84.

Hordon, M.J. \& Averbach, B.L. (1961). X-ray measurements of dislocation density in deformed copper and aluminum single crystals. Acta Metallurgica 9(3), 237-246.

Hosaka, M. \& Taki, S. (1981). Hydrothermal growth of quartz crystals in pure water. Journal of Crystal Growth 51(3), 640-642.

Huang, Y., Lieber, C.M., et al. (2002). Gallium nitride nanowire nanodevices. Nano Letters 2 (2), 101-104.

Im, J. S., et al. (1997). Radiative carrier lifetime, momentum matrix element, and hole effective mass in GaN. Applied Physics Letters 70(5), 631-633.

Iwahashi, T., et al. (2003). Effects of ammonia gas on threshold pressure and seed growth for bulk GaN single crystals by Na flux method. Journal of Crystal Growth 253 (14), 1-5. 
Izumi, T., Nakamura, S., et al. (2000). Time-resolved photoluminescence spectroscopy in GaN-based semiconductors with micron spatial resolution. Journal of Luminescence 87-89, 1196-1198.

Jensen, L.E., Samuelson, L., et al. (2004). Role of surface diffusion in chemical beam epitaxy of InAs nanowires. Nano Letters 4(10), 1961-1964.

Jian, J.K., et al. (2003). Large-scale GaN nanobelts and nanowires grown from milled $\mathrm{Ga}_{2} \mathrm{O}_{3}$ powders. Chemical Physics Letters 368(3-4), 416-420.

Johnson, J.C. et al. (2002). Single gallium nitride nanowire lasers. Nature Materials 1(2), $106-110$.

Johnson, J.C., Yan, H.Q., Yang, P.D. \& Saykally, R.J. (2003). Optical cavity effects in $\mathrm{ZnO}$ nanowire lasers and waveguides. Journal of Physical Chemistry B 107(34), 8816-8828.

Jouet, R.J., et al. (2002). Preparation of phase pure cubic gallium nitride, c-GaN, by ammonothermal conversion of gallium imide, $\{\mathrm{Ga}(\mathrm{NH})(3 / 2)\}(\mathrm{n})$. Journal of Cluster Science13(4), 469-486.

Kadinski, L., et al. (2004). Computational analysis of GaN/InGaN deposition in MOCVD vertical rotating disk reactors. Journal of Crystal Growth 261(2-3),175-181.

Kamler, G., et al. (2000). Bulk GaN single-crystals growth. Journal of Crystal Growth 212(1-2), 39-48.

Kapolnek, D., Mishra, U. K., et al. (1997). Anisotropic epitaxial lateral growth in GaN selective area epitaxy. Applied Physics Letters 71(9), 1204-1206.

Karpinski, J., et al. (1984). Equilibrium pressure of $\mathrm{N}_{2}$ over $\mathrm{GaN}$ and high pressure solution growth of GaN. Journal of Crystal Growth 66(1), 1-10.

Karpinski, J. \& Porowski, S. (1984). High pressure thermodynamics of GaN. Journal of Crystal Growth 66(1), 11-20.

Kern, W. \& Deckert, C.A. (1976). Chemical Etching, Thin Film Processes. in Handbook of semiconductor wafer cleaning techniques. Ed. J. Vossen \& W. Kern, Academic Press, NY, 401-496.

Ketchum, D.R. \& Kolis, J.W. (2001). Crystal growth of gallium nitride in supercritical ammonia. Journal of Crystal Growth 222(3), 431-434.

Khan, M.A. \& Shur, M.S. (1997). GaN based transistors for high temperature applications. Materials Science and Engineering B 46(1-3), 69-73.

Khan, M.A., Kuznia, J.N., Olson, D.T., Kaplan R. (1993). Deposition and surface characterization of high quality single crystal GaN layers. Journal of Applied 
Physics 73(6), 3108-3110.

Kidoguchi, I., et al. (2000). Improvement of crystalline quality in $\mathrm{GaN}$ films by airbridged lateral epitaxial growth. Japanese Journal of Applied Physics 39(5B), L453-L456.

Kim, S.T., et al. (1998). Preparation and properties of free-standing HVPE grown GaN substrates. Journal of Crystal Growth 194(1), 37-42.

Kisielowski, C., et al. (1996). Strain-related phenomena in GaN thin films. Physical Review B 54(24), 17745-17753.

Kitamura, S., Hiramatsu, K., Sawaki, N. (1995). Fabrication of GaN hexagonal pyramids on dot-patterned $\mathrm{GaN} /$ sapphire substrates via selective metalorganic vapor phase epitaxy. Japanese Journal of Applied Physics Part 2. 34(9B), L1184-L1186.

Klemenz, C. \& Scheel, H.J. (1998). Crystal growth and epitaxy from solutions I: Fundamentals of growth from solutions. Materials Science Forum 276-277, 175190.

Knacke, O., Kubaschewski, O. \& Hesselmann, K. (1991). Thermochemical properties of inorganic substances. Springer, Berlin.

Kocha, S.S., Turner, J.A., et al. (1995). Electrochemical investigation of the gallium nitride-aqueous electrolyte interface. Journal Of The Electrochemical Society 142(12), L238-L240.

Koleske, D.D., et al. (1998). Growth model for GaN with comparison to structural, optical, and electrical properties. Journal of Applied Physics 84(4), 1998-2010

Koleske, D.D., Wickenden, A.E., Henry, R.L. (2000). GaN decomposition in ammonia. MRS Internet Journal of Nitride Semiconductor Research 5S1, Art. No.W3.64.

Koleske, D.D., et al. (2001). GaN decomposition in $\mathrm{H}_{2}$ and $\mathrm{N}_{2}$ at MOVPE temperatures and pressures. Journal of Crystal Growth 223(4), 466-483.

Korona, K. P., et al. (1999). Excitonic thermalization and recombination in homoepitaxial gallium nitride. Physica Status Solidi (b) 216(1), 85-89.

Kozawa, T., et al. (1994). Raman scattering from LO phonon-plasmon coupled modes in gallium nitride. Journal of Applied Physics 75(2), 1098-1101.

Krukowski, S., Romanowski, Z., Grzegory, I., Porowski, S. (1998). Interaction of $\mathrm{N}_{2}$ molecule with liquid Ga surface - quantum mechanical calculations (DFT). Journal of Crystal Growth 189/190, 159-162.

Krukowski, S., Leszcynski, M. \& Porowski, S. (1999). In: Properties, Processing and Applications of Gallium Nitride and Related Semiconductors, Eds. J.H. Edgar, S.S. 
Strite, I. Akasaki, H. Amano. INSPEC, The Institution of Electrical Engineers, Stevenage, UK, 21.

Krukowski, S., (1999). Growth of GaN single crystals under high nitrogen pressures and their characterization. Crystal Res. Technol. 34(5-6), 785-795.

Kucheyev, S.O., et al. (2000). Nanoindentation of epitaxial GaN films. Applied Physics Letters 77(21), 3373-3375.

Kuykendall, T., Yang, P.D., et al. (2004). Crystallographic alignment of high-density gallium nitride nanowire arrays. Nature Materials 3(8), 524-528.

Kwon, H.K., et al. (2000). Time-resolved photoluminescence study of GaN grown by metalorganic chemical vapor deposition. Journal of Crystal Growth 221(1-4), 240245.

Kwon, Y., et al. (2000). Time-resolved study of yellow and blue luminescence in Si- and Mg-doped GaN. Applied Physics Letters 76(7), 840-842.

Leszczynski, M., et al. (1999). GaN homoepitaxial layers grown by metalorganic chemical vapor deposition. Applied Physics Letters 75(9), 1276-1278.

Levinshtein, M.E., Rumyantsev, S.L. \& Shur, M.S. (2001). Properties of Advanced Semiconductor Materials: GaN, AlN, InN, BN, and SiGe, John Wiley and Sons, New York.

Li, H. \& Sunkara, M.K. (2003). Growth of oriented gallium nitride films on amorphous substrates by self-assembly. MRS Symposium Proceedings 743, L3.11.

Li, H., et al. (2005). Self-oriented growth of GaN films on molten gallium. MRS Symposium Proceedings 831, E11.34.

Li, Z.J., et al. (2001). Synthesis and raman scattering of GaN nanorings, nanoribbons and nanowires. Applied Physics A-Materials Science \& Processing 72(5), 629-632.

Lin, T.Y., Yang, H.C., Chen, Y.F. (2000). Optical quenching of the photoconductivity in n-type GaN. Journal of Applied Physics 87(7), 3404-3408.

Lintenberg, M.K., Weber, M.J. \& Derenzo, S.E. (2003). Luminescence and scintillation of $\mathrm{PbI}_{2}$ and $\mathrm{HgI}_{2}$. Journal of Luminescence 102-103, 287-290.

Liu, L. \& Edgar, J.H. (2002). Substrates for gallium nitride epitaxy. Materials Science and Engineering $R$ 37(3), 61-127.

Logan, R.A. \& Thurmond, C.D. (1972). Heteroepitaxial thermal gradient solution growth of GaN. The Journal of The Electrochemical Society 119(12), 1727-1735.

Madar, R., et al. (1975). High pressure solution growth of GaN. Journal of Crystal 
Growth 31, 197-203.

Marchand, H., et al. (1999). Structural and optical properties of GaN laterally overgrown on $\mathrm{Si}(111)$ by metalorganic chemical vapor deposition using an AlN buffer layer. MRS Internet Journal of Nitride Semiconductor Research 4, 2.

Maruska, H.P., \& Tietjen, J.J. (1965). The preparation and properties of vapor-deposited single-crystal-line GaN. Applied Physics Letters 15(10), 327-329.

McGregor, D.S. \& Hermon, H. (1997). Room-temperature compound semiconductor radiation detectors. Nuclear Instruments and Methods in Physics Research A 395(1), 101-124.

Miedema, A.R. \& Broeder, F.J.A. (1979). Interfacial energy in solid-liquid and solidsolid metal combinations. Zeitschrift Fur Metallkunde 70(1), 14-20.

Mingo, N. (2004). Thermoelectric figure of merit of II-VI semiconductor nanowires. Applied Physics Letters 85(24), 5986-5988

Miyake, H., et al. (2002). Carrier-gas dependence of ELO GaN grown by hydride VPE. Journal of Crystal Growth 237-239, 1055-1059.

Mohammad, S. N., Salvador, A.A \& Morkoc, H. (1995). Emerging gallium nitride based devices. Proceedings of The IEEE 83(10), 1306-1355.

Molnar, R.J., Singh, R., Moustakas, T.D. (1995). Operation of a compact electron cyclotron resonance source for the growth of $\mathrm{GaN}$ by MBE. Journal of Electronic Materials 24(4), 275-281.

Monemar, B., et al. (1997). Optical characterization of GaN and related materials. Solid State Electronics 41(2), 181-184.

Moore, D., Wang, Z.L., et al. (2004). Wurtzite ZnS nanosaws produced by polar surfaces. Chemical Physics Letters 385 (1-2), 8-11.

Morkoc, H. (2001). Comprehensive characterization of hydride VPE grown GaN layers and templates. Materials Science and Engineering R 33(5-6), 135-207.

Moriarty, P. (2001). Nanostructured materials. Reports on Progress in Physics 64(3), 297-381.

Motoki, K., et al. (2002). Growth and characterization of freestanding GaN substrates. Journal of Crystal Growth 237-239(2), 912-921.

Murr, L.E. (1974). Interfacial Phenomena in Metals and Alloys. Addison Wesley Publication Company, New York.

Nakamura, S., et al. (1991) Novel metalorganic chemical vapor deposition system for 
GaN growth. Applied Physics Letters 58(18), 2021-2023.

Nakamura, S. (1991). GaN growth using GaN buffer layer. Japanese Journal of Applied Physics Part 2-Letters 30(10A), L1705-L1707.

Nakamura, S., et al. (1992). Thermal annealing effects on P-type Mg-doped GaN films. Japanese Journal of Applied Physics 31(2B), L139-142.

Nakamura, S. \& Fasol, G. (1997). The blue laser diode: GaN based light emitters and lasers. Springer-Verlag, Heidelberg.

Nam, O., Davis, R.F., et al. (1997). Lateral epitaxy of low defect density GaN layers via organometallic vapor phase epitaxy. Applied Physics Letters 71(18), 2638-2640.

Neugebauer, J., Feenstra, R.M., et al. (2003). Adatom kinetics on and below the surface: the existence of a new diffusion channel. Physical Review Letters 90(5), Art. No. 056101 .

Nikitina, I.P., Dmitriev, V.A., et al. (1997). Residual strains in GaN grown on 6H-SiC. Diamond and Related Materials 6(10), 1524-1527.

Ning, X. J., et al. (1996). Growth defects in GaN films on sapphire: The probable origin of threading dislocations. Journal of Materials Research 11(3), 580-592.

Northrup J.E. \& Neugebauer J. (1996). Theory of GaN(10-10) and (11-20) surfaces. Physical Review B 53 (16), 10477-10480.

Oberman, D. B. (1995). Molecular beam epitaxy of gallium nitride by electron cyclotron resonance plasma and hydrogen azide. Journal of Crystal Growth 150(2), 912-915.

Ogino, T. \& Aoki, M. (1980). Mechanism of yellow luminescence in GaN. Japanese Journal of Applied Physics 19(12), 2395-2405.

Ohba, Y. \& Hatano, A. (1994). H-atom incorporation in Mg-doped GaN grown by metalorganic chemical-vapor-deposition. Japanese Journal of Applied Physics Part 2-Letters 33(10A), L1367-L1369.

Ohshima, E., et al. (2003). Growth of the 2-in-size bulk $\mathrm{ZnO}$ single crystals by the hydrothermal method. Journal of Crystal Growth 260(1-2), 166-170.

Okada, K. \& Ozone, H. (1992). Experimental heat transfer rates of natural convection of molten gallium suppressed under an external magnetic field in either $\mathrm{X}, \mathrm{Y}$ or $\mathrm{Z}$ direction. Journal of Heat Transfer 114(1), 107-114.

Pakula, K., Porowski, S., et al. (1996). Luminescence and reflectivity in the exciton region of homoepitaxial GaN layers grown on GaN substrates. Solid State Communications 97(11), 919-922. 
Pankove, J.I. (1971). Optical Processes in Semiconductors, Prentice-Hall Inc., Englewood, NJ.

Pashley, D.W., et al (1964). The growth and structure of gold and silver deposits formed by evaporation inside an electron microscope. Philosophical Magazine 10, 127-158.

Pashley, D.W. (1965). The nucleation, growth, structure and epitaxy of thin surface films. Advances in physics 14, 327-410.

Pavlovska, A. \& Bauer, E. (2001). Wetting and reactive thin film growth. Surface Science 480(3), 128-136.

Pawlowski, R.P., et al. (2000). Fundamental models of the metalorganic vapor-phase epitaxy of gallium nitride and their use in reactor design. Journal of Crystal Growth 221(1-4), 622-628.

Pearson, W.B. (1967). A handbook of Lattice Spacings and Structures of Metals and Alloys, Vol. 2, Pergamon Press, Oxford, London, 1.

Perlin, P., Porowski, S., et al. (1995). Investigation of longitudinal-optical phononplasmon coupled modes in highly conducting bulk GaN. Applied Physics Letters 67(17), 2524-2526.

Perry, W. D., Bremser, M. B., Davis, R. F. (1998). Cathodoluminescence studies of the deep level emission bands of $\mathrm{Al}_{x} \mathrm{Ga}_{1-} \mathrm{N}$ films deposited on $6 \mathrm{H}-\mathrm{SiC}(0001)$. Journal of Applied Physics 83(1), 469-475.

Pezzagna, S., et al. (2004). Polarity inversion of $\mathrm{GaN}(0001)$ by a high $\mathrm{Mg}$ doping. Journal of Crystal Growth 269(2-4), 249-256.

Ponce, F.A., Bour, D.P., Young, W.T., Saunders, M., Steeds, J.W. (1996). Determination of lattice polarity for growth of GaN bulk single crystals and epitaxial layers. Applied Physics Letters 69(3), 337-339.

Porowski, S. (1997). Growth and properties of single crystalline GaN substrates and homoepitaxial layers. Materials Science and Engineering B 44(1-3), 407-413.

Porowski, S. \& Grzegory, I. (1997). Thermodynamical properties of III-V nitrides and crystal growth of $\mathrm{GaN}$ at high $\mathrm{N}_{2}$ pressure. Journal of Crystal Growth 178(1-2), 174-188.

Prywer, J. \& Krukowski, S. (1998). GaN single crystal habits and their relation to gan growth under high pressure of nitrogen. MRS Internet Journal of Nitride Semiconductor Research 3, 47.

Purdy, A.P., Case, S., \& Muratore, N. (2003). Synthesis of GaN by high-pressure ammonolysis of gallium triiodide. Journal of Crystal Growth 252(1-3), 136-143. 
Purdy, A.P., Jouet, R.J., George, C.F. (2002). Ammonothermal recrystallization of gallium nitride with acidic mineralizers. Crystal Growth \& Design 2(2), 141-145.

Purdy, A.P. (1999) Ammonothermal synthesis of cubic gallium nitride. Chemistry of Materials 11(7), 1648-1651.

Raghothamachar, B., et al. (2002). Synchrotron white beam topography characterization of physical vapor transport grown AlN and ammonothermal GaN. Journal of Crystal Growth 246(3-4), 271-280.

Ramachandran, V., Feenstra, R.M., et al. (2000). Optimized structural properties of wurtzite $\mathrm{GaN}$ on $\mathrm{SiC}(0001)$ grown by molecular beam epitaxy. Journal of Vacuum Science \& Technology A18(4), 1915-1918.

Rodina, A.V., Meyer, B.K., et al. (2001). Free excitons in wurtzite GaN. Physical Review B 64(11), 115204-115208.

Rouviere, J.L., et al. (1996). Correlation between surface morphologies and crystallographic structures of GaN layers grown by MOCVD on sapphire. MRS Internet Journal of Nitride Semiconductor Research 1, Art. No. 33.

Ruterana, P., et al. (1997). The structure of GaN layers grown on $\mathrm{SiC}$ and sapphire by molecular beam epitaxy. Materials Science and Engineering B 50(1-3), 72-75.

Scheiber, C. \& Giakos, G.C. (2001). Medical applications of CdTe and CdZnTe detectors. Nuclear Instruments and Methods in Physics Research A 458(1-2), 12-25.

Schotanus, P., Dorenbos, P.\& Ryzhikov, V.D. (1992). Detection of CdS(Te) and $\mathrm{ZnSe}(\mathrm{Te})$ scintillation light with silicon photodiodes. IEEE Transactions on Nuclear Science NS 39(4), 546-550.

Sharma, S. \& Sunkara, M.K. (2004). Direct synthesis of single-crystalline silicon nanowires using molten gallium and silane plasma. Nanotechnology 15(1), 130134.

Sheka, I.A., Chaus, I.S. \& Mityureva, T.T. (1966). Chemistry of Gallium. Elsevier Publishing Company, London.

Shi, W.S., Lee, S.T., et al. (2001). Microstructures of gallium nitride nanowires synthesized by oxide-assisted method. Chemical Physics Letters 345(5-6), 377-380.

Shin, H., Z. Sitar, et al. (2002) High temperature nucleation and growth of GaN crystals from the vapor phase. Journal of Crystal Growth 241(4), 404-415.

Shin, H., Sitar, Z., Davis, R.F., et al. (2002). Growth and decomposition of bulk GaN: role of the ammonia/nitrogen ratio. Journal of Crystal Growth 236(4), 529-537.

Shur, M.S., Bykhovski, A.D., Gaska, R. (1999). Pyroelectric and piezoelectric properties 
of GaN-based materials. MRS Internet Journal of Nitride Semiconductor Research 4S1, No. G1.6.

Smart, J.A., et al. (1999). Single step process for epitaxial lateral overgrowth of GaN on SiC and sapphire substrates. Applied Physics Letters 75(24), 3820-3822.

Song, Y.T., et al. (2004). Preparation and characterizations of bulk GaN crystals, Journal of Crystal Growth 260(3-4), 327-330.

Steranka, F.M., et al. (2002). High power LEDs - technology status and market applications. Physica Status Solidi (a) 194(2), 380-388.

Stocker, D.A, Schubert, E.F., Redwing, J.M. (1998). Crystallographic wet chemical etching of GaN. Applied Physics Letters 73(18), 2654-2656.

Strategies Unlimited Report. (2005) Gallium Nitride 2005 - Technology Status, Applications, and Market Forecasts.

Sunkara, M.K., et al., (2001). Bulk synthesis of silicon nanowires using low temperature vapor-liquid-solid method. Applied Physics Letters 79(10), 1546-1548.

Tabata, A., et al. (1996). Comparative Raman studies of cubic and hexagonal GaN epitaxial layers. Journal of Applied Physics 79(8), 4137-4140.

Tiginyanu, I.M., et al. (2003). Luminescence of $\mathrm{GaN}$ nanocolumns obtained by photonassisted anodic etching. Applied Physics Letters 83(8), 1551-1553.

Tsang, W.T. (1984). Chemical beam epitaxy of InP and GaAs. Applied Physics Letters 45(11), 1234-1236.

Tijburg, R. (1976). Advances in etching of semiconductor devices, Physics in Technology, 202-207.

Trivedi, M. \& Shenai, K. (1999). Performance evaluation of high-power wide band-GaP semiconductor rectifiers. Journal of Applied Physics 85(9), 6889-6897.

Vaddiraju, S., et al. (2005). Mechanisms of 1D crystal growth in reactive vapor transport: Indium nitride nanowires. Nano Letters, in press.

Van Vechten, J.A. (1973). Quantum dielectric theory of electronegativity in covalent systems III: pressure-temperature phase diagrams, heats of mixing, and distribution coefficients. Physical Review B 7(4), 1479-1507.

Wade, K. \& Banister, A.J. (1975). The Chemistry of Aluminium, Gallium, Indium and Thallium. Pergamon Press, Oxford, New York.

Waltereit, P. (2000). Nitride semiconductors free of electrostatic fields for efficient white light-emitting diodes. Nature 406(6798), 865-868. 
Wang, H.X., et al. (2000). Growth of GaN layer by metal-organic chemical vapor deposition system with a novel three-flow reactor. Journal of Crystal Growth 218(2-4), 148-154.

Warren, R. (1980). Solid-liquid interfacial energies in binary and pseudo-binary systems. Journal of Materials Science 15(10), 2489-2496.

Wetzel, C., et al. (1996). Carrier localization of as-grown n-type gallium nitride under large hydrostatic pressure. Physical Review B 53(3), P1322-1326.

Weyher, J.L., Grzegory, I., et al. (2000). Recent advances in defect-selective etching of GaN. Journal of Crystal Growth 210(1-3), 151-156.

Weyher, J.L., Grzegory, I., et al. (2003). Characterization of GaN single crystals by defect-selective etching. Physica Status Solidi (c) vol.0(3), 821-826.

White, C.E.T. \& Okamoto, H. (1992). Phase Diagrams of Indium Alloys and their Engineering Applications, Monograph Series on Alloy Phase Diagrams, 1st edition, ASM International, Materials Park, $\mathrm{OH}$.

Widmann, F., et al. (1999). Low temperature sapphire nitridation: a clue to optimize GaN layers grown by molecular beam epitaxy. Journal of Applied Physics 85(3), 15501555.

Wilcox, W.R. (1983). Influence of convection on the growth of crystals from solution. Journal of Crystal Growth 65(1-3), 133-142.

Wilson W.L., Szajowski P.F. \& Brus L.E. (1993). Quantum confinement in size-selected, surface-oxidized silicon nanocrystals. Science 262 (5137), 1242-1244.

Xu, H.Z., Foxon, C.T., et al. (2001). Competition between band gap and yellow luminescence in undoped GaN grown by MOVPE on sapphire substrate. Journal of Crystal Growth 222(1-2), 96-103.

Yamane, H., et al. (1997). Preparation of GaN single crystals using a Na flux. Chemistry of Materials 9(2), 413-416.

Yamane, H., et al. (1998). Morphology and characterization of GaN single crystals grown in a Na flux. Journal of Crystal Growth 186(1-2), 8-12.

Yamane, H., et al. (2000). Growth of zinc-blend-type structure GaN from a Na-Ga melt. Materials Letters 42(1-2), 66-70.

Yamane, H., et al. (2002). Fabrication of cubic gallium nitride single crystal using a KGa melt. Journal of the Ceramic Society of Japan 110(4), 289-292.

Yamanaka, Y., Kakimoto, K., Ozoe, H. \& Churchill, S.W. (1998). Rayleigh-benard oscillatory natural convection of liquid gallium heated from below. Chemical 
Engineering Journal 71(3), 201-205.

Yano, M., et al. (2000). Growth of nitride crystals, BN, AlN and GaN by using a Na flux. Diamond and Related Materials 9(3-6), 512-515.

Yao, Y.M. \& Tholen, A. (2002). Characterization of contacting boundaries between small particles with microdiffraction. Nanotechnology 13(2), 169-174.

Young, T. (1805). An essay on the cohesion of fluids. Philosophical Transactions of the Royal Society of London Series A 95, 65-87.

Yu, G., et al. (1997). Optical properties of wurtzite structure GaN on sapphire around fundamental absorption edge $(0.78-4.77 \mathrm{eV})$ by spectroscopic ellipsometry and the optical transmission method. Applied Physics Letters 70(24), 3209-3211.

Zauner, A.R.A., et al. (2002). Homo-epitaxial growth on the $\mathrm{n}$-face of $\mathrm{GaN}$ single crystals: The influence of the misorientation on the surface morphology. Journal of Crystal Growth 240(1-2), 14-21.

Zheleva, T.S., Davis, R.F., et al. (1999). Thermal mismatch stress relaxation via lateral epitaxy in selectively grown GaN structures. Applied Physics Letters 74(17), 24922494.

Zuo, J. M. \& J. C. Mabon, WebEMAPS software, University of Illinois at UrbanaChampaign; URL: http://emaps.mrl.uiuc.edu/

http://www.ioffe.ru/SVA/NSM/Semicond/GaN/

http://education.jlab.org/itselemental/ele031.html 


\section{APPENDIX I}

\section{GALLIUM NITRIDE ON SINGLE CRYSTALLINE SAPPHIRE SUBSTRATES}

\section{AND ITS SCITILLATING PROPERTIES}

\section{A1.1 Introduction}

Self-oriented GaN crystals have been grown on molten gallium with amorphous quartz substrates [Li \& Sunkara, 2003]. As mentioned earlier, this technique is independent of substrates since the GaN crystals are formed on top of molten gallium. Therefore we introduce single crystal sapphire substrates to demonstrate this point by nitridation of molten gallium films using atomic nitrogen.

\section{A1.2 Experimental Results}

\section{A1.2.1 GaN on Ga VS epitaxial GaN directly on Sapphire}

The experimental procedures and conditions for growth with sapphire substrates are the same as those of synthesis on amorphous quartz substrates. The initial gallium films on sapphire substrates agglomerated into droplets with considerable size distribution and then spread after exposed to nitrogen plasma at high temperatures. The surface profile of the spread gallium was not uniform due to the uncontrolled flow pattern. Two different zones of gallium nitride formation were found after the experiments, one is the self-oriented $\mathrm{GaN}$ on top of molten gallium, and the other is $\mathrm{GaN}$ directly on sapphire substrates without Ga buffer layer. The SEM images of these two different zones are shown in Fig. A1.1. 


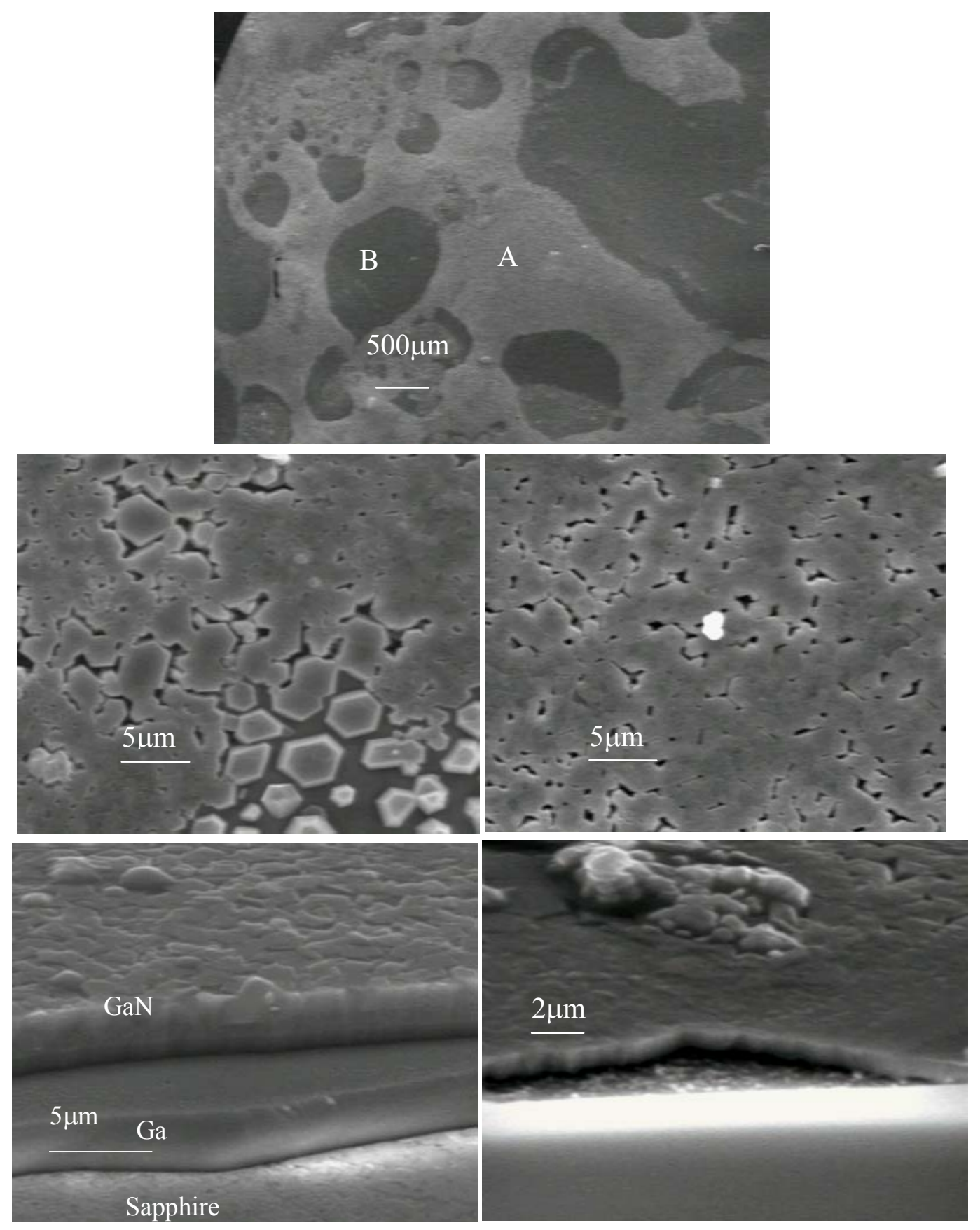

Figure A1.1 Plan view and cross sectional view of two different zones of $\mathrm{GaN}$ on sapphire substrates. (a) $\mathrm{GaN}$ on $\mathrm{Ga}$ (b) $\mathrm{GaN}$ directly on sapphire. 


\section{A1.2.2 Sequential Growth of GaN on Ga}

The growing and coalescing of the GaN platelets on top of molten gallium are clearly shown in Fig. A1.2.

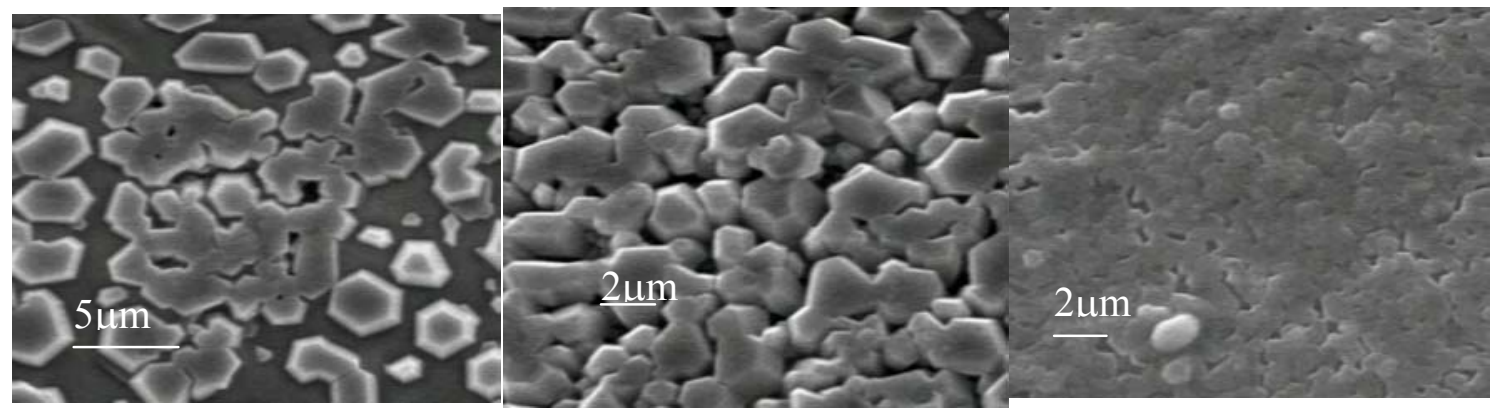

Figure A1.2 Growing and coalescing of GaN crystals on Ga with sapphire substrates.

\section{A1.3 Structure Properties}

\section{A1.3.1 X-ray Diffraction}

Powder XRD analysis was performed using a Rigaku x-ray diffractometer (Model VS-DXR3000) with standard $\mathrm{Cu} \operatorname{K} \alpha$ radiation $(\lambda=1.54 \AA)$. The $\theta-2 \theta$ scan of XRD shown in Fig. A1.3 indicates the synthesized GaN with no post treatment is c-plane oriented. The split of peaks is due to diffractions from copper $K \alpha_{1}$ and $K \alpha_{2}$ radiations since the intensity is very strong. The peak at $2 \theta=31.5$ is tentatively attributed to $\mathrm{Ga}_{2} \mathrm{O}_{3}$. However, in the absence of other $\mathrm{Ga}_{2} \mathrm{O}_{3}$ peaks we couldn't make a conclusive remark, other possible complex phases are possible. 


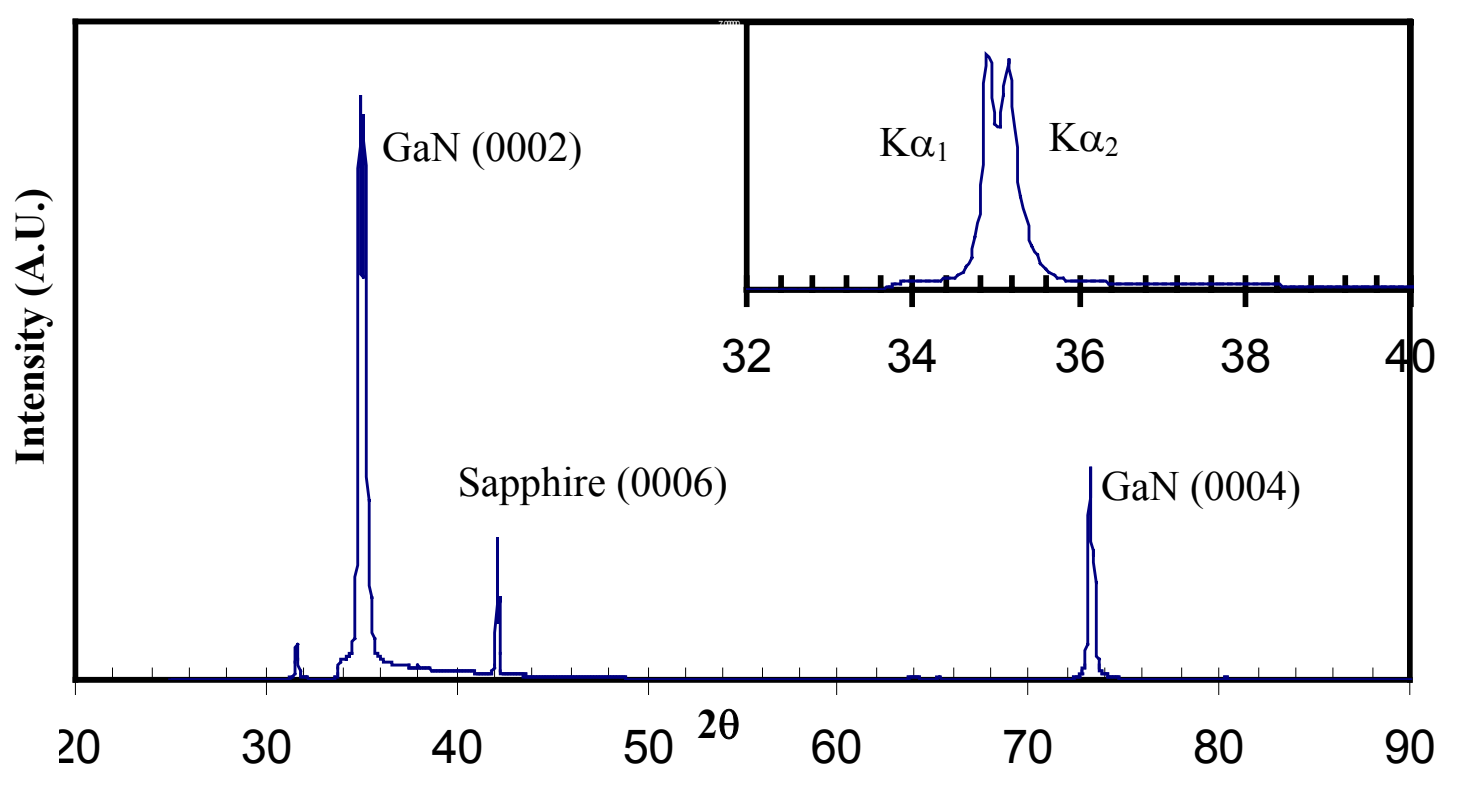

Figure A1.3 XRD spectra of GaN with sapphire substrates indicating c-plane orientation.

\section{A1.3.2 Synchrotron Radiation Tomography of GaN directly on Sapphire}

The synchrotron radiation tomography experiments were carried out at the

Synchrotron Radiation Tomography experimental station 2.2 at the Stanford Synchrotron Radiation Laboratory (SSRL), Stanford, CA. Laue diffraction technique using white beam radiation was employed to obtain the transmission tomographs of the synthesized $\mathrm{GaN}$ after dissolution of excess $\mathrm{Ga}$ with aqua regia. The incident beam was aligned parallel to the [0001] direction of the GaN crystals and the images were recorded on high-resolution Kodak SR1 type films for all topographic work. A completely automated Philips high-resolution diffractometer with incident beam optics consisting of fourbounce Ge (220) Bartels monochromator was used to study the X-ray rocking curves of (0002) and $(10 \overline{1} 2)$ planes to evaluate the overall quality of the crystal.

The composite Laue diffraction pattern using the Synchrotron white beam x-ray (SWBX) from the GaN film on sapphire substrate is shown in Fig. A1.4(a). The data in 
Fig. A1.4(a) indicate that GaN film is single crystalline and it is in epitaxial orientation relationship with the substrate. This is expected since the GaN on top of Ga with no epitaxial relationship with sapphire was washed off after the Ga was dissolved away. The orientation relationship between the $\mathrm{GaN}$ and the sapphire substrate is found to be (0001) $\mathrm{GaN} / /(0001)$ sapphire and $(1 \overline{1} \overline{2} 0) \mathrm{GaN} / /(1 \overline{1} 00)$ sapphire. The simulated Laue diffraction patterns of (0001) GaN and (0001) sapphire are also shown in Fig. A1.4 (c) and (d) for comparison. Shown in Fig. A1.4(b) is the enlarged image of one of the GaN spots in Fig. A1.4(a) and indicates cellular structures of GaN crystals. The FWHM of the $\mathrm{x}$-ray rocking curve of (1012) reflection from this sample shows the best results of 949 arcsec. The results on four different samples are listed in Table A1.1. In order to capture the effect of all type of dislocations (screw, edge and mixed) the (10 $\overline{1} 2)$ reflecting plane, which is at an angle of approximately $43.8^{\circ}$ with respect to the (0001) growth plane, was chosen for x-ray rocking curve analysis [Heying, B., Speck, J.S., et al., 1996]. A rough estimate of dislocation density, D, was obtained from the full width at half maximum (FWHM) of the rocking curve of the (1012) reflection and using the following equation [Hordon \& Averbach, 1961]:

$$
D=(F W H M / \sqrt{\pi})^{2} /\left(4.36 b^{2}\right)
$$

Where $\mathrm{b}$ is the burgers vector along the $[11 \overline{2} 0]$ direction. The FWHM of the $\mathrm{x}$-ray rocking curve of $(10 \overline{12})$ reflection from the sample shows 949 arcsec which corresponds to a dislocation density of $6 \times 10^{9} / \mathrm{cm}^{2}$. The high density of dislocation is expected since no buffer layer was intentionally applied. 
Table A1.1 Rocking curve of 4 different samples.

\begin{tabular}{|l|l|l|}
\hline \multirow{2}{*}{ Sample \# } & \multicolumn{2}{|l|}{$\begin{array}{l}\text { Full Width at Half Maximum (FWHM) } \\
\text { at different reflection }(\operatorname{arcsec})\end{array}$} \\
\cline { 2 - 3 } & $(0002)$ & - \\
& & $(1012)$ \\
\hline No.1 & 1106 & 1171 \\
\hline No.2 & 955 & 1090 \\
\hline No.3 & 648 & 949 \\
\hline No.4 & 1570 & 1799 \\
\hline
\end{tabular}

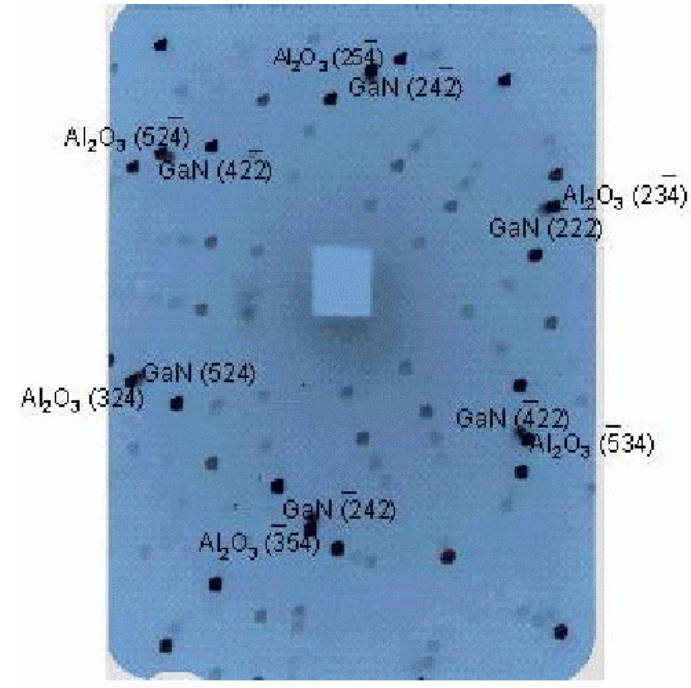

(a)

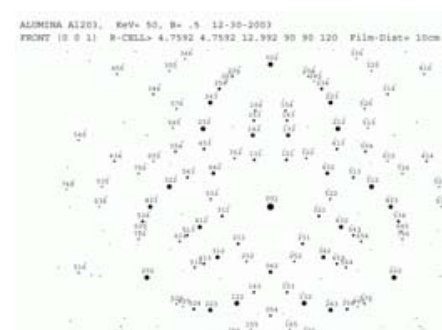

(c)

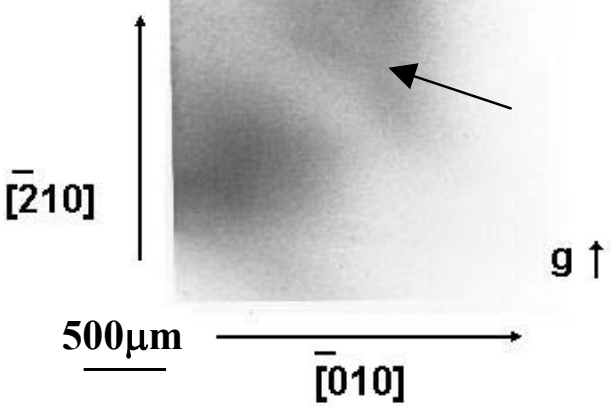

(b)

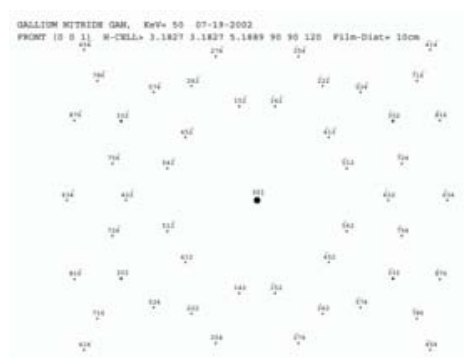

(d)

Figure A1.4 (a) Synchrotron white beam x-ray transmission Laue diffraction pattern from GaN film on sapphire, exposure time $10 \mathrm{sec}$ and specimen to film distance $10 \mathrm{~cm}$. (b) Enlarged one of the diffraction spot in (b), $g=24 . \overline{2}$, wavelength $=0.2657 \AA, \mathrm{A}=$ cellular structure of GaN crystals. (c) Simulated Laue DP of GaN, (0001) face, $50 \mathrm{KeV}$, 
film distance $=10 \mathrm{~cm}$. (d) Simulated Laue DP of Sapphire, (0001) face, $50 \mathrm{KeV}$, film distance $=10 \mathrm{~cm}$.

\section{A1.4 Optical Properties}

\section{A1.4.1 Raman Spectroscopy}

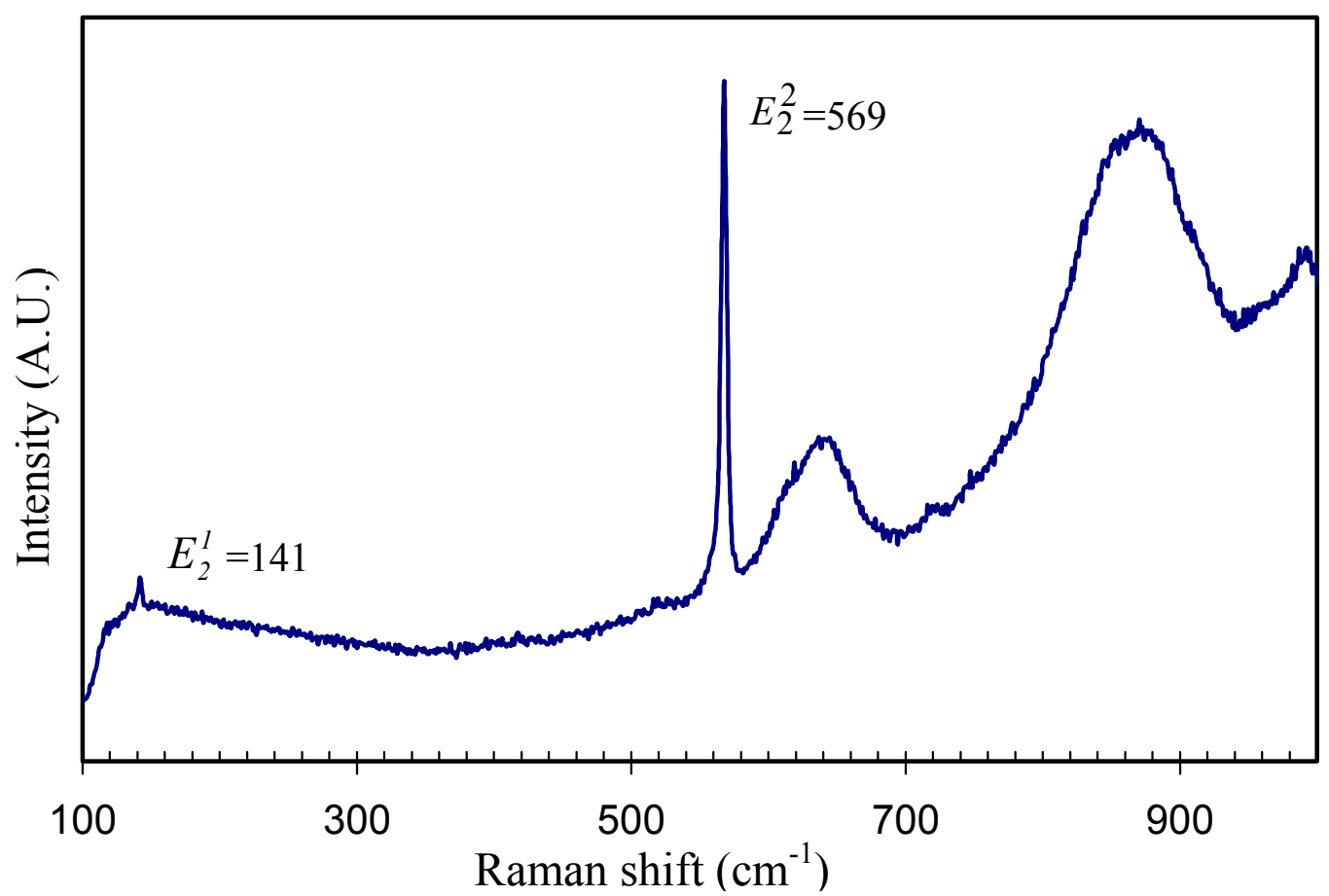

Figure A1.5 Raman spectrum of GaN on sapphire in back scattering configuration indicates wurtzite phase. The two broad bumps on the right side are from the sapphire substrates.

Raman spectrum in back-scattered configuration under which only $E_{2}$ and $A_{1}$ (LO) modes are allowed for hexagonal GaN [Tabata, A., et al., 1996] is shown in Fig. A1.5. The shift of $\mathrm{E}_{2}^{2}$ mode from defect-free position of $567 \mathrm{~cm}^{-1}$ to high frequency of $569 \mathrm{~cm}^{-1}$ indicate that it is in compressive stress since sapphire has a higher thermal expansion coefficient than that of GaN [Kisielowski, C., et al., 1996]. The disappearance of $A_{1}(\mathrm{LO})$ mode is believed to the results of plasmon-phonon interactions [Demangeot, 
F., et al., 1997] with electron concentration exceeding $10^{19} \mathrm{~cm}^{-3}$ which is confirmed by Hall-effect measurement. The high donor concentration might come from the diffusion of oxygen from the sapphire substrates during the growth.

\section{A1.4.2 Catholuminescence and Scintillation of GaN}

Direct wide-bandgap semiconductors such as ZnO [Derenzo, S. E., et al., 2002], CdS [Schotanus, P., et al., 1992], CdTe [Scheiber \& Giakos, 2001], $\mathrm{HgI}_{2}$ and $\mathrm{PbI}_{2}$ [Lintenberg, M. K., et al., 2003] have been found to be useful as scintillating materials due to their ultra-fast (sub-nanosecond) decay time and relatively high luminosity. Conductivity radiation detectors have been made out of these compound semiconductors [McGregor \& Hermon, 1997]. An excellent review on the development of inorganic scintillators and understandings of their scintillating mechanism has been published by a group at Lawrence Berkeley National Laboratory [Derenzo, S. E., et al., 2003]. Here, we investigated the scintillating properties of low-pressure, bulk synthesized gallium nitride on sapphire substrates.

\section{A1.4.2.1 Scintillation Properties Measurement Setup}

The cathodoluminescence studies were performed at El-Mul Technologies, Ltd and the efficiency was examined using an electron gun with electron energy of $8 \mathrm{KeV}$. The cathodoluminescence gain, defined as the ratio of photo-multiplier tube (PMT) signal to the electron gun current, was compared to a calibrated standard scintillator of El-Mul Technologies Ltd., placed adjacent to the samples under test. The CL PMT signal was measured in a backscattered configuration with a UV pass filter and without the filter. The time response properties of the samples were measured using electron bursts from chevron micro-channel-plates (MCP) and photon counting system shown in Fig. A1.6. 
The photon counting setup is configured around "HARP", a photon counting system of PicoQuant GMBH. Each electron burst from the MCPs produces a multi-photon avalanche in PMT - 1 and its rise time onset is registered by the HARP relative to the rise time onset produced by single photon received by PMT-2. HARP provides the time histogram of the single photons received by PMT-2 as a function of the registered time difference from the pulse received at PMT-1. HARP is providing a minimum histogram channel width of $40 \mathrm{ps}$. Although the specified response (FWHM) of the chevron MCPs is $\sim 450 \mathrm{ps}$, the time accuracy of the histogram is determined by the jitter of the produced pulse after being processed by a constant fraction discriminator and is better than $75 \mathrm{ps}$. It should be noted that while the area of the e-beam excitation for the measurements of CL spectra is about $0.3 \mathrm{~mm}$ by $0.3 \mathrm{~mm}$, the excitation area for the time response measurement is typically over a larger area of $\sim 1 \mathrm{~cm} \times 1 \mathrm{~cm}$.

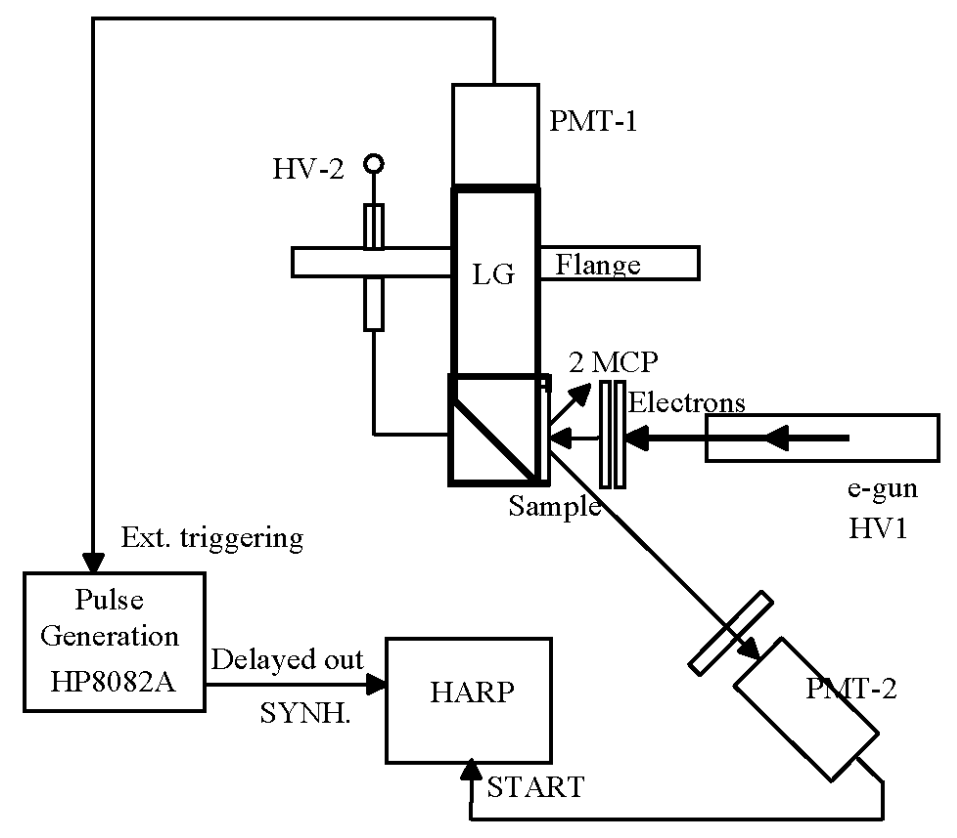

Figure A1.6 Photon counting setup for time-resolved CL measurement. HV-High Vacuum, LG-Light guide, MCP-micro-channel plate, PMT-photo multiplier. 


\section{A1.4.2.2 Cathodoluminescence}

The sample with strong UV emission shows an integrated intensity ratio of 2.9:1 between the UV and whole wavelength emission. The integrated intensity of whole wavelength emission for the sample with dominating yellow luminescence is about 7 times higher than that for UV emission only. The CL UV efficiency of the sample with dominating UV emission was estimated to be about 950 photons/ $\mathrm{MeV}$ based on the calibration of the standard scintillator of El-Mul Technologies placed close to the sample.

The spatial maps of the cathodoluminescence PMT intensity taken with a UV band pass filter positioned at the PMT light input port for the samples are shown in Fig. A1.7. Spatial non-uniformity of the UV luminescence is attributed to the non-uniform gallium film during growth process [Li \& Sunkara, 2003]. The CL spectra from two different areas in the sample with dominating UV emission indicated as " 1 " and " 2 " in Fig. A1.7(a) are shown in Fig. A1.8. The spectra show a small yellow luminescence around $2.35 \mathrm{eV}$ and a relatively intense band centered at $3.38 \sim 3.39 \mathrm{eV}$. The FWHM of the near band edge emission is about $120 \mathrm{meV}$ for curve " 1 " and $140 \mathrm{meV}$ for curve " 2 ", respectively. The CL spectrum of region " 3 " in Fig. A1.7(b) in the sample with dominating yellow luminescence is also shown in Fig. A1.8. The intensity of the yellow luminescence is about 10 times higher than that of near band-edge emission. 


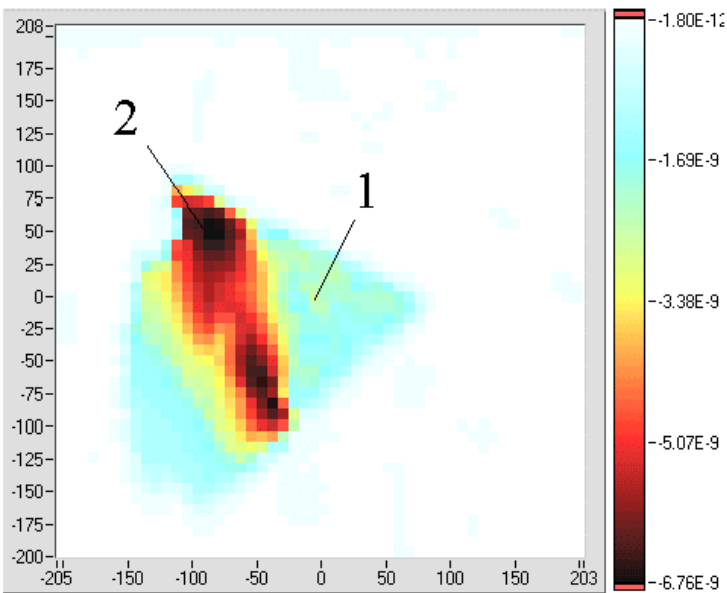

(a)

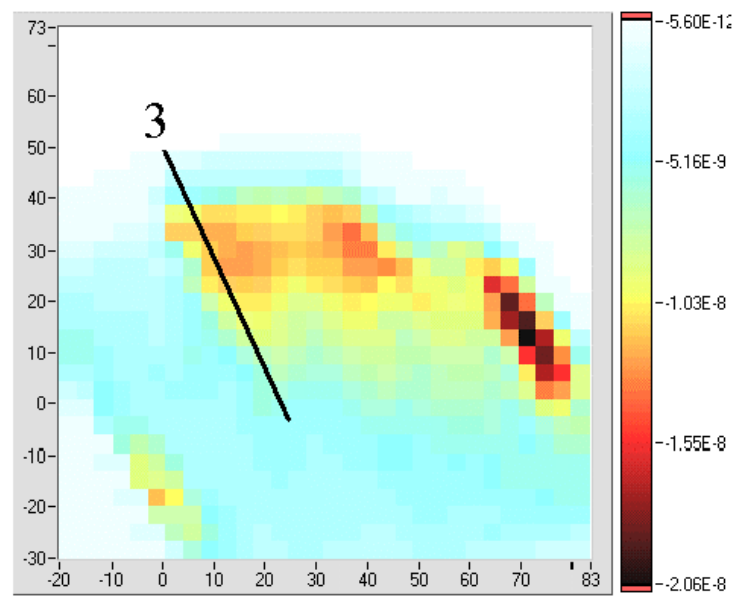

(b)

Figure A1.7 The CL intensity map of the GaN samples with UV filters at room temperature. (a) UV photoluminescence dominating sample (b) Yellow luminescence dominating sample. The bar on the right side denotes the PMT current.

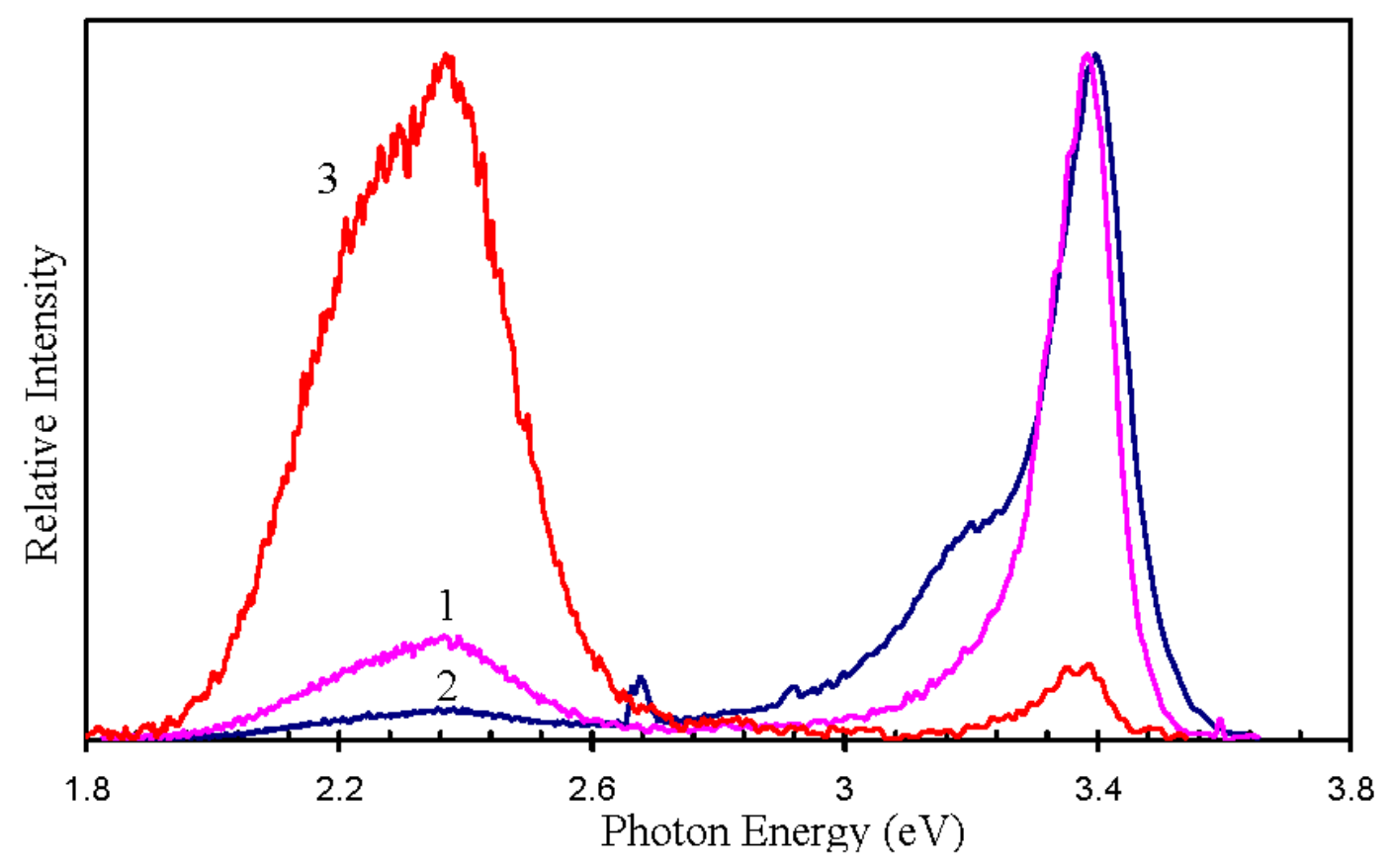

Figure A1.8 Normalized CL spectra of GaN from different regions shown in Fig. A1.7; (1) and (2): with dominating near band edge emission; (3) with dominating yellow luminescence. 


\section{A1.4.2.3 Time-resolved Cathodoluminescence}

Fig. A1.9 shows the overall response time of GaN on sapphire with dominating UV emission measured with and without UV filter at room temperature. To determine the exponential decay time and fractions, the function of time response is convolved with a sum of three components according to the method in Ref. [Schotanus, P., et al., 1992].

$$
I=\frac{W_{1}}{\tau_{1}} \exp \left(-\frac{t}{\tau_{1}}\right)+\frac{W_{2}}{\tau_{2}} \exp \left(-\frac{t}{\tau_{2}}\right)+\frac{W_{3}}{\tau_{3}} \exp \left(-\frac{t}{\tau_{3}}\right)
$$

Where, $\tau$ is the decay time of each component, $\mathrm{W}$ is the weight of each component; The region with dominant $\mathrm{UV}$ band emission has a rise time of $0.3 \mathrm{~ns}$, which is slower than the reported rise time for similar wide bandgap semiconductor $\mathrm{ZnO}$ :Ga powder sample [Derenzo, S. E., et al., 2002]. This might indicates a morphological impact on the scintillation mechanism which is not clear at present. The decay time for the three components are $0.33 \mathrm{~ns}, 2.5 \mathrm{~ns}, 50 \mathrm{~ns}$ and their weights are $0.31,0.125$, and 0.25 , without filter respectively for the spectrum from the sample with dominating UV emission without filter. The fitting curve is also shown in Fig. A1.9. The reports on the decay time of $\mathrm{GaN}$ at room temperature have been few and the values of fastest decay components range from 22 ps to 150 ps [Ahn, S.H., et al., 2002; Kwon, H. K., et al., 2000]. These data are hard to compare with each other since these materials are from different processes and the dopants are varying. The fastest decay time of $0.33 \mathrm{~ns}$ is the longest ever reported for GaN under room temperature at author's knowledge. The fastest component is believed to be the band-to-band transition and the longest decay component is related to the remains of the yellow band recombination centers. The second part might come from the bound defects related with donor since the sample has a higher donor concentration on the order of $10^{19} / \mathrm{cm}^{3}$. The temperature dependent decay time studies are 
currently being conducted to further understand the scintillation mechanism and the results will be presented in another paper.

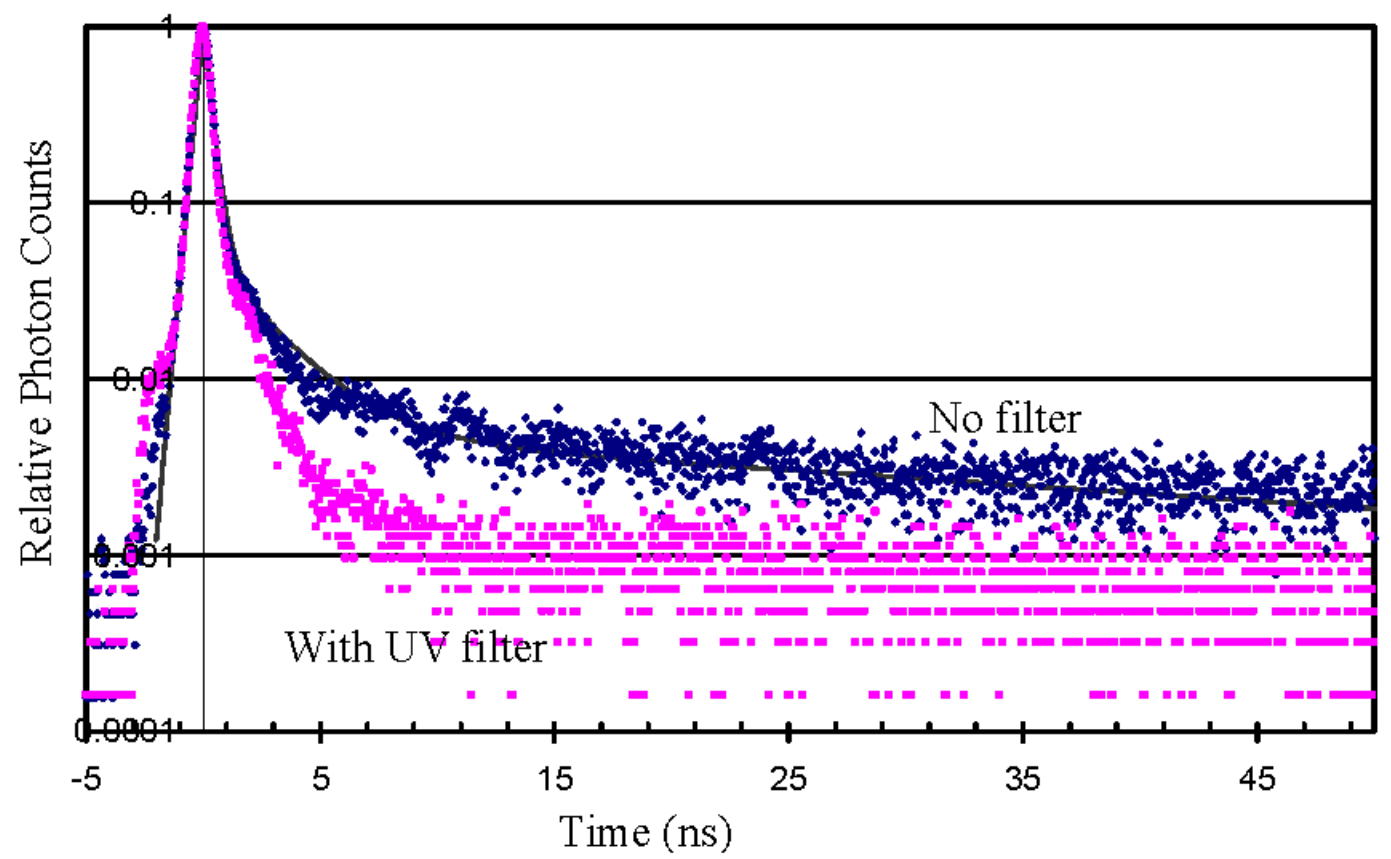

Figure A1.9 Response time of GaN on sapphire with dominating UV emission measured with and without UV filter at room temperature, the line is the fitted curve of data.

The band-edge emission of $\mathrm{GaN}$ is related to radiative recombination of bound and free excitons [Pakula, K., et al., 1996; Korona, K. P., et al., 1999; Rodina, A.V., et al., 2001]. The dominating radiative process at room temperature is believed to be free exciton recombination [Monemar, B., et al., 1997]. The low luminosity of the sample at room temperature means the nonradiative recombination process is dominating [Izumi, T., et al., 2000] and dislocations have been confirmed to act as nonradiative recombination centers [Dassonneville, S., et al., 2001; Cherns, D., et al., 2001]. The luminosity of the GaN has to be improved at least one order of magnitude for practical applications. The high density of dislocations can be reduced to decrease the nonradiative recombination centers at room temperature by engineering the growth processes. 
There are both radiative and nonradiative recombination processes in the decay of excited carriers. The effective recombination time of excited carriers in semiconductors is governed by:

$$
\frac{1}{\tau}=\frac{1}{\tau_{r}}+\frac{1}{\tau_{n r}}
$$

Where, $\tau$ is the effective (i.e. measured) recombination time, $\tau_{r}$ is the radiative decay time and $\tau_{n r}$ is called the non-radiative decay time. The quantum efficiency is given by [Pankove, J. I., 1971]:

$$
\eta=\frac{W_{r}}{W_{r}+W_{n r}}=\frac{1 / \tau_{r}}{1 / \tau_{r}+1 / \tau_{n r}}
$$

Where $W_{r}$ and $W_{n r}$ are radiative recombination rate and non-radiative recombination rate, respectively. By combining equations (A1.3) and (A1.4), there is:

$$
\eta=\frac{\tau}{\tau_{r}}
$$

In our case, the radiative decay time could be directly deduced since we have a direct measurement of the quantum efficiency. Near band edge emission dominates in our sample and $\eta$ is estimated to be around 0.003 using the standard scintillator and $\tau$ is between $0.33 \mathrm{~ns}$ and $2.5 \mathrm{~ns}$. The estimation of the efficiency is based on the assumption that the light from $\mathrm{GaN}$ has the same form as that of standard scintillator and is a lower limited value. Therefore the deduced radiative decay time at room temperature is on the order of tens of ns and compares well with the reported value [Im, J. S., et al., 1997; Cazzanelli, M., et al., 1998].

The extrinsic yellow luminescence (YL) $(\sim 2.3 \mathrm{eV})$ is found in $\mathrm{GaN}$ samples grown by different techniques. The presence of the YL band is not well understood and is 
attributed to various sources such as $\mathrm{N}$ vacancy [Glaser, E. R., et al., 1995], N antisite [Lin, T. Y., et al., 2000], Ga vacancy [Basak, B., et al., 1999], Ga interstitial [Kwon, Y., et al., 2000], impurities [Ogino \& Aoki, 1980], or crystal defects [Freitas, J. A., et al., 1998; Marchand, H., et al., 1999; Chen, Z., et al., 2000]. The cellular structures of the film and the dislocations in the sample might be the origin of the yellow luminescence. Different recombination mechanism of YL has also been proposed, including shallow donors to deep acceptors [Perry, W. D., et al., 1998], deep intrinsic double donor to shallow acceptors [Glaser, E. R., et al., 1995], shallow donors to deep donors [Kwon, Y., et al., 2000]. Recently, a more deep investigation of recombination mechanism of YL has also been presented [Xu, H. Z., et al., 2001]. Time resolved photoluminescence (PL) of yellow luminescence showed a decay time up of 50ns for a heavily Si-doped sample at room temperature [Kwon, Y., et al., 2000], which is comparable with our data and much longer than that of band edge emission.

\section{A1.5 Summary}

Direct nitridation of thin film gallium over single crystal sapphire substrates resulted in self-oriented films which are partially in epitaxy with underlying substrates. The overall film exhibited the same orientation with gallium buffer layer between GaN and substrates in certain regions. The cathodoluminescence efficiency and response time of oriented GaN crystals on sapphire has been investigated. The near band-edge emission gives a conversion efficiency of about 950 photons per MeV electrons. Three decay time components are found, of which the faster components are due to band-to-band transition and the longest decay component is related to the remains of the yellow band recombination centers. The radiative decay time at room temperature is estimated on the 
order of tens of ns. GaN with cellular structures might have caused extrinsic yellow luminescence. 


\section{APPENDIX II}

\section{NANOCRYSTALLINE GALLIUM NITRIDE FILMS ON AMORPHOUS QUARTZ SUBSTRATES}

\section{A2.1 Introduction}

Large area oriented GaN crystals had been synthesized using Ga films on amorphous quartz substrates. The thicknesses of Ga films for these experiments are usually more than $100 \mu \mathrm{m}$. Preliminary results on studying relationship of nucleation density and Ga thickness have shown that nucleation density is higher with decreasing Ga thickness. Here we studied the nitridation of Ga film less than $2 \mu \mathrm{m}$ on quartz substrates. Spin-coating has been widely used to make thin film such as photoresist in microfabrication process. High surface tension liquid metal such as gallium (melting point $29.3{ }^{\circ} \mathrm{C}, 700 \mathrm{mN} / \mathrm{m}$ at $25^{\circ} \mathrm{C}$ ) hasn't been reported to form thin films using spincoating. Amorphous quartz was chosen as the substrates since they are relatively cheap, transparent and have good mechanical strength. Also, dislocations are not expected due to the amorphous nature of the substrates. The using of nitrogen plasma is also environmental friendly and safe for experimental operation.

\section{A2.2 Experimental}

Liquid gallium was found that it could stick to the glass substrates and forms a film by pressing using external force at room temperature in air. Utilizing this property, less than 2 microns thick gallium was spin-coated on 2-inch quartz wafers. The nitridation experiments were performed under same conditions as oriented GaN growth. 
The synthesized gallium nitride films were characterized using X-ray Diffraction (XRD), high-resolution transmission electron microscopy (HR-TEM) and reflective UVVis spectroscopy. XRD was performed using a Rigaku Model VS-DXR3000 X-ray diffractometer with standard $\mathrm{Cu} \mathrm{K} \alpha$ radiation $(\lambda=1.54056 \AA)$. High resolution TEM analysis was done on a JEOL 2010 operated at $200 \mathrm{kV}$. UV-Vis spectrometer was used to study the band gap of the GaN film.

\section{A2.3 Result and Discussion}

Less than $2 \mu \mathrm{m}$ thick gallium was coated on a 2 -inch quartz wafer by spinning (Shown in Fig. A2.1(a)). A yellowish thin film formed on the substrate after nitridation in ECR-MW generated nitrogen plasma (Shown in Fig. A2.1 (b)). SEM pictures in Fig. A2.1(c) showed the size of the crystallites in the film was around $85 \mathrm{~nm}$. X-ray diffraction in Fig. A2.2 indicates the film is textured. The possible reason of forming nanocrystalline $\mathrm{GaN}$ is the simultaneous, high-density nucleation due to thinness of molten Ga film.
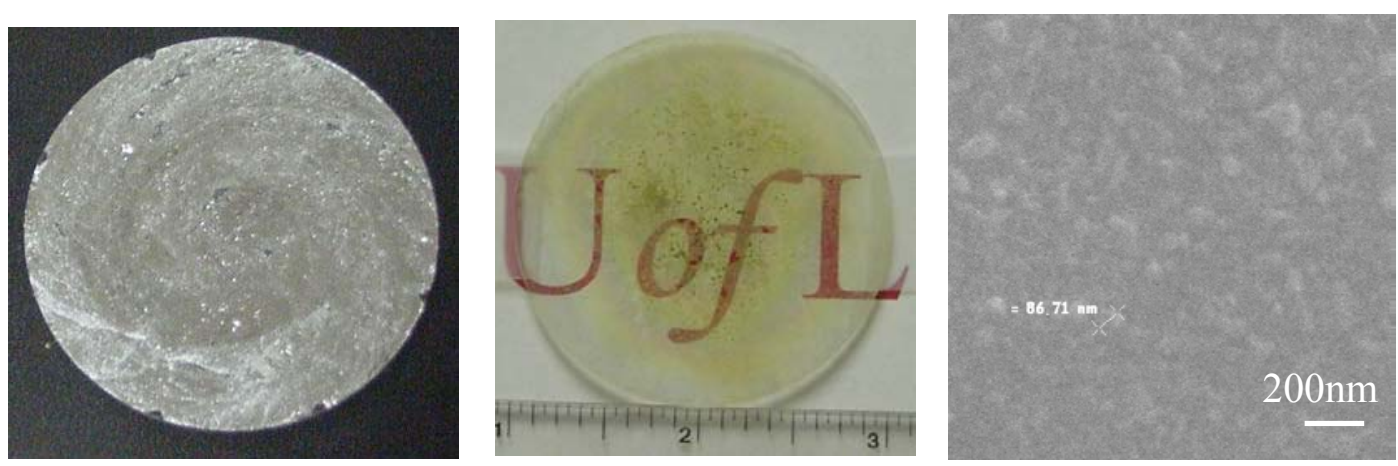

Figure A2.1 (a) Optical micrograph of spun coated gallium film on 2-inch quartz substrate. (b) Optical micrograph of the as-nitrided substrate. (c) SEM image of nanocrystalline GaN film showing crystallite size of $85 \mathrm{~nm}$. 


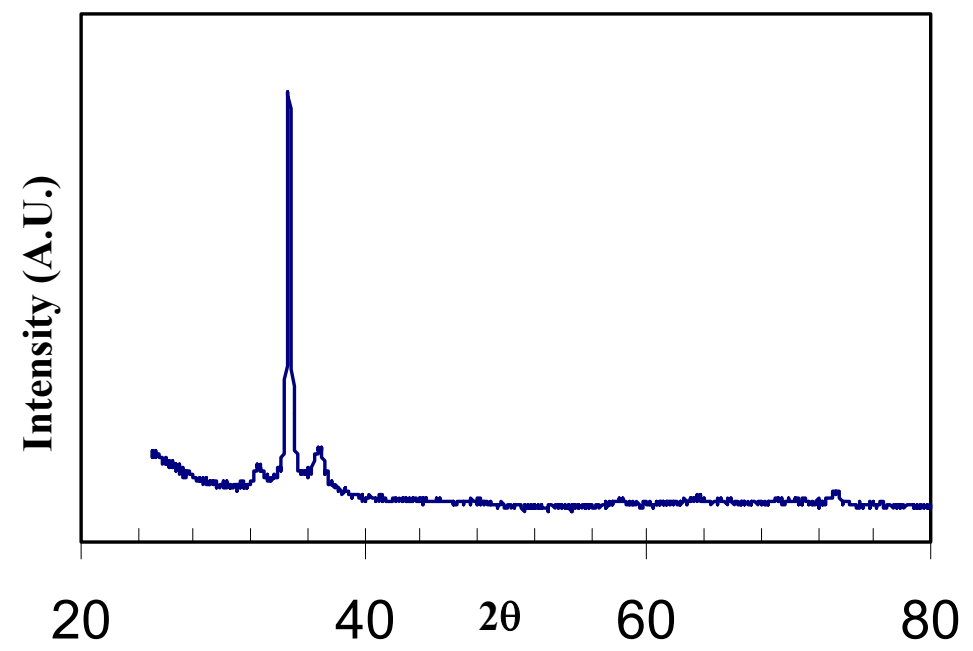

Figure A2.2 XRD of textured nanocrystalline GaN showing dominate (0002) peak of wurtzite phase.

Cross sectional TEM images of the film were shown in Fig. A2.3 (a). The thickness of the film was about $500 \mathrm{~nm}$. The high-resolution TEM image of the quartzGaN sharp interface shown in Fig. A2.3 (b) indicates no epitaxial relationship with the substrates.

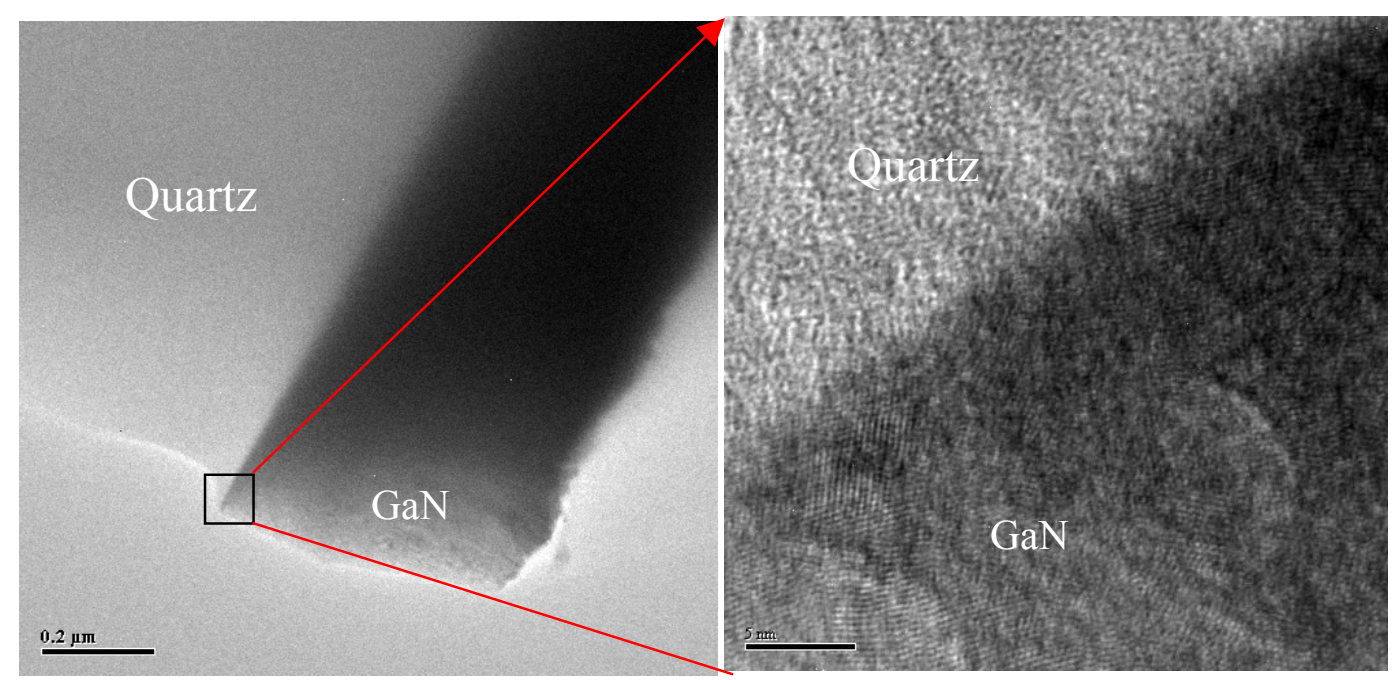

Figure A2.3 Cross sectional TEM images of nanocrystalline $\mathrm{GaN}$ film on quartz substrate showing sharp interface of amorphous quartz and GaN. 
TEM micrographs and their corresponding selected area diffraction (SAD)

patterns of the film with different growth recycle were shown in Fig. A2.4. Fig. A2.4(a) is the film with one cycle of growth whose SAD pattern showing almost polycrystalline of the grains. While SAD pattern of the film with four cycles of growth in Fig. A2.4(b) indicates that the grains mainly have several orientations. These results indicate that the GaN grain size increased and the films became textured after further growth.

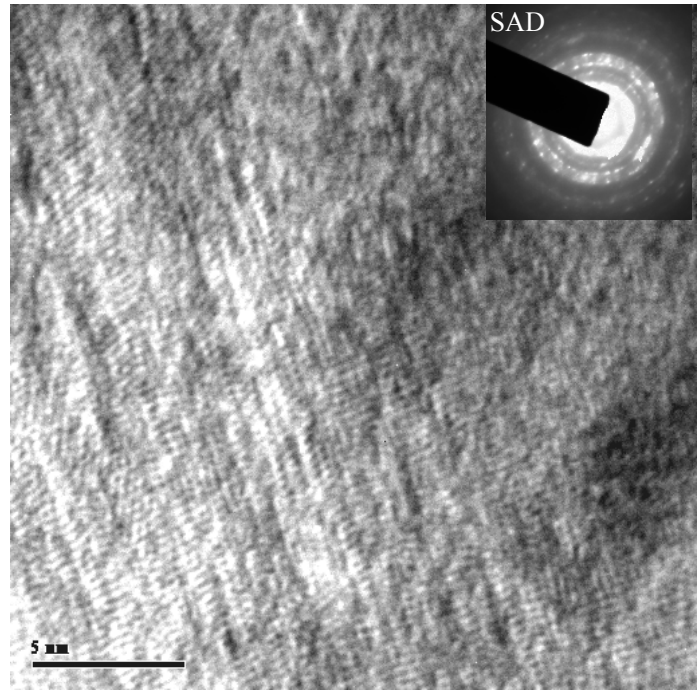

(a)

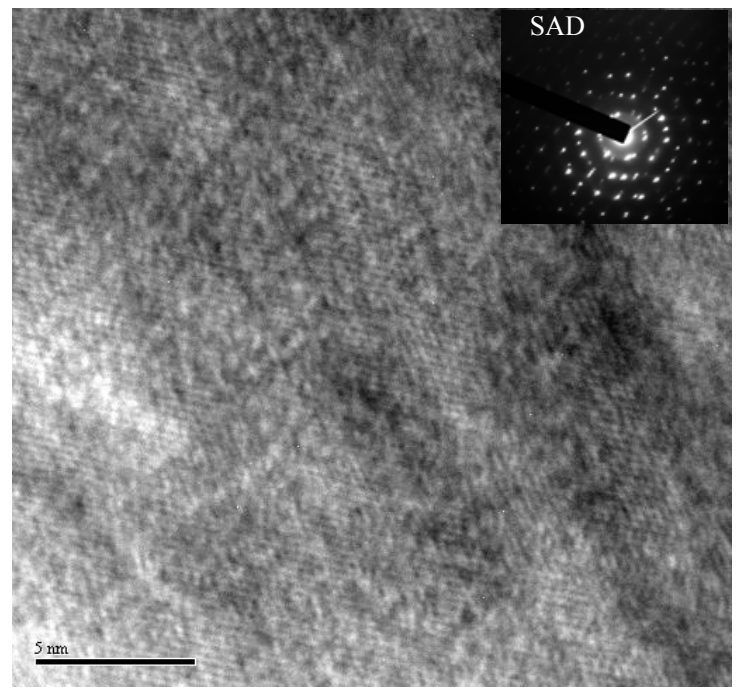

(b)

Figure A2.4 Cross section TEM micrographs of nanocrystalline GaN with regrowth show increased grain size (a) one cycle of growth (b) four cycles of growth.

The optical transmission spectrum of GaN nanocrystalline thin film on quartz at room temperature was shown in Fig. A2.5. The spectra were obtained with background scan using blank quartz substrates. The spectrum in Fig. A2.5(a) is the absorbance of the nanocrystalline film with respect to the wavelength of the light. Fig. A2.5(b) shows the plot of transmittance versus wavelength of light. The transmission is shown to increase above wavelength of $340 \mathrm{~nm}$. The periodic modulation shown in transmittance plot is due to the interference within the GaN film. 
The band gap of materials can be derived by using the relationship between absorption coefficient $\alpha$ and photon energy (hv) of the incident light, i.e. the Tauc equation [Pankove, 1971]:

$$
\alpha^{2}=\left(h v-E_{g}\right) /(h v)^{2}
$$

Where the absorption coefficient $\alpha$ can be obtained by:

$$
\alpha=-d^{-1} \ln \left\{<A+\left[A^{2}+2 B T\left(1-R_{2} R_{3}\right)\right]^{0.5}>/ B\right\}
$$

Where $A=-\left(1-R_{1}\right)\left(1-R_{2}\right)\left(1+R_{3}\right), B=2 T\left(R_{1} R_{2}+R_{1} R_{3}-2 R_{1} R_{2} R_{3}\right), d$ is the thickness of the film, $T$ is the transmittance, $R_{1}, R_{2}, R_{3}$ are reflectances of the air-film, film-substrate, and substrate-air interfaces, respectively. The reflectances from a boundary between two media 1 and 2 may be calculated using the equation:

$$
R_{12}=\left[\left(n_{2}-n_{1}\right) /\left(n_{2}+n_{1}\right)\right]^{2}
$$

Where $\mathrm{n}_{1}, \mathrm{n}_{2}$ are the refractive indices of media 1 and 2 , respectively. The values of $\mathrm{n}$ were taken as 2.33 for GaN [Yu, G., et al., 1997] and 1.54 for quartz substrates. The plot of $(\alpha \times h \gamma)^{2}$ to $h \gamma$ is shown in Fig. A2.5(b). The band gap $E_{g}$ for the GaN film was extrapolated to be $3.45 \mathrm{eV}$. 


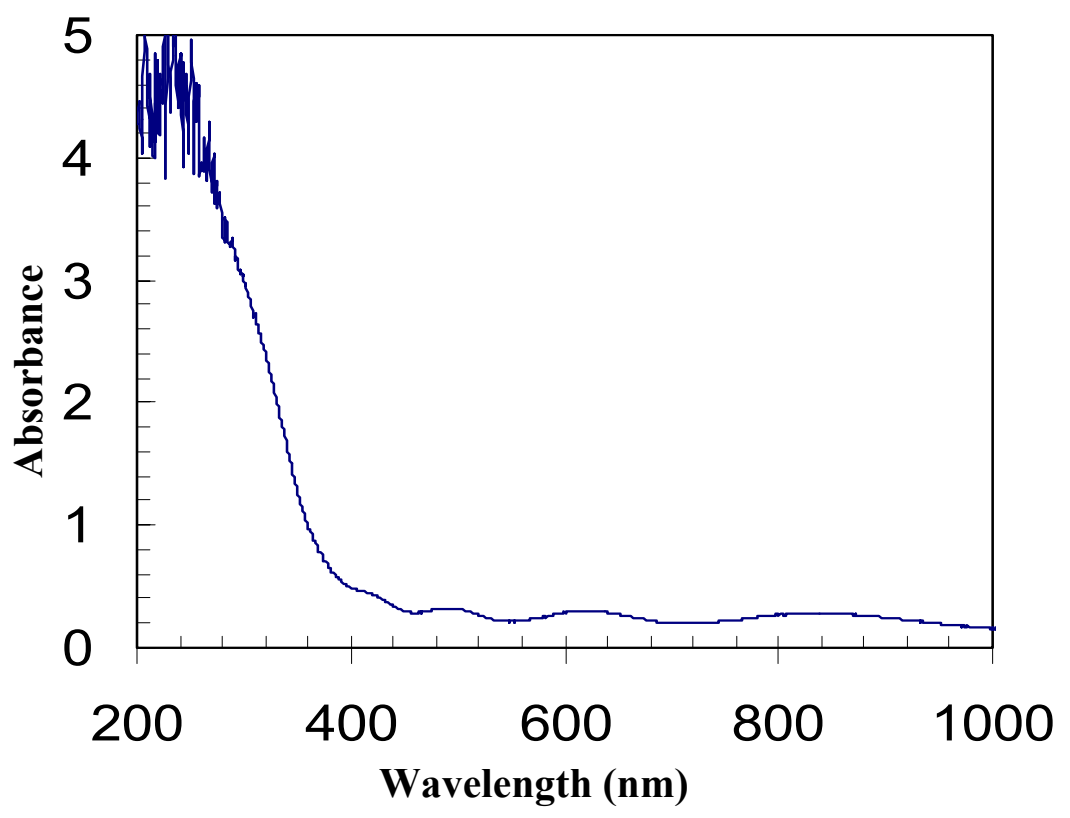

(a)

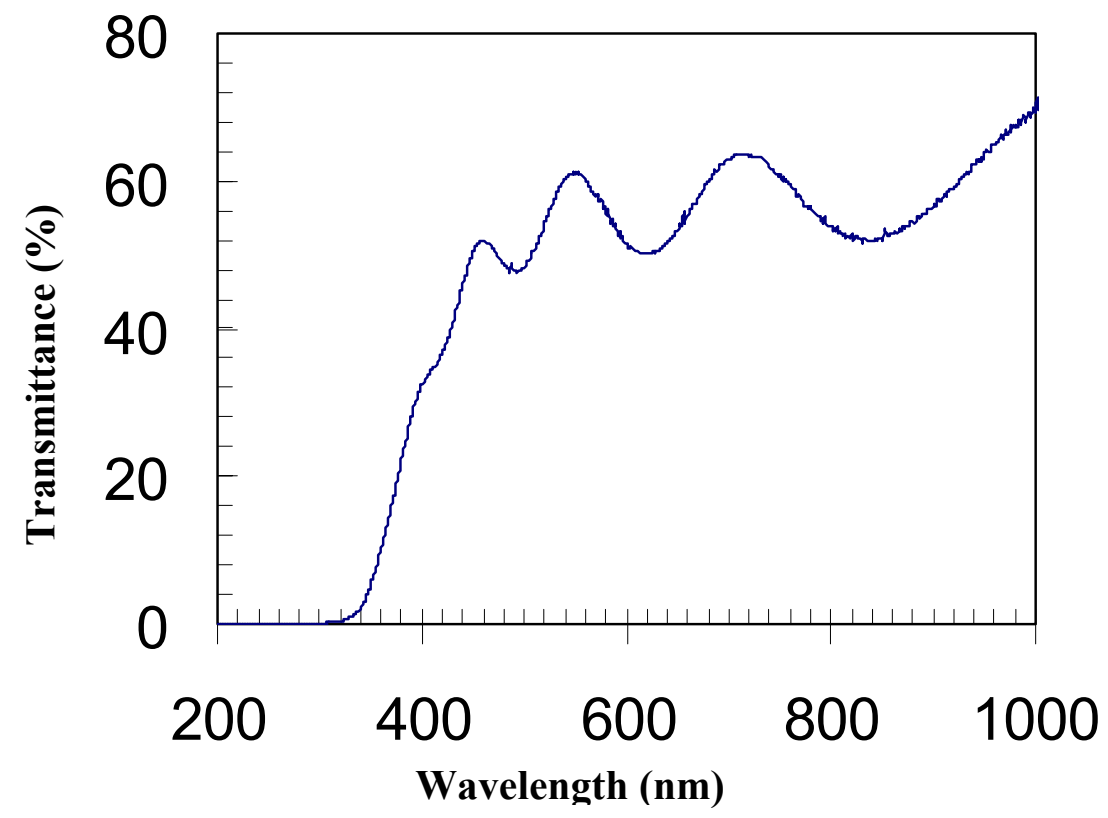

(b)

Figure A2.5 UV-Vis spectra of nanocrystalline GaN on amorphous quartz. 


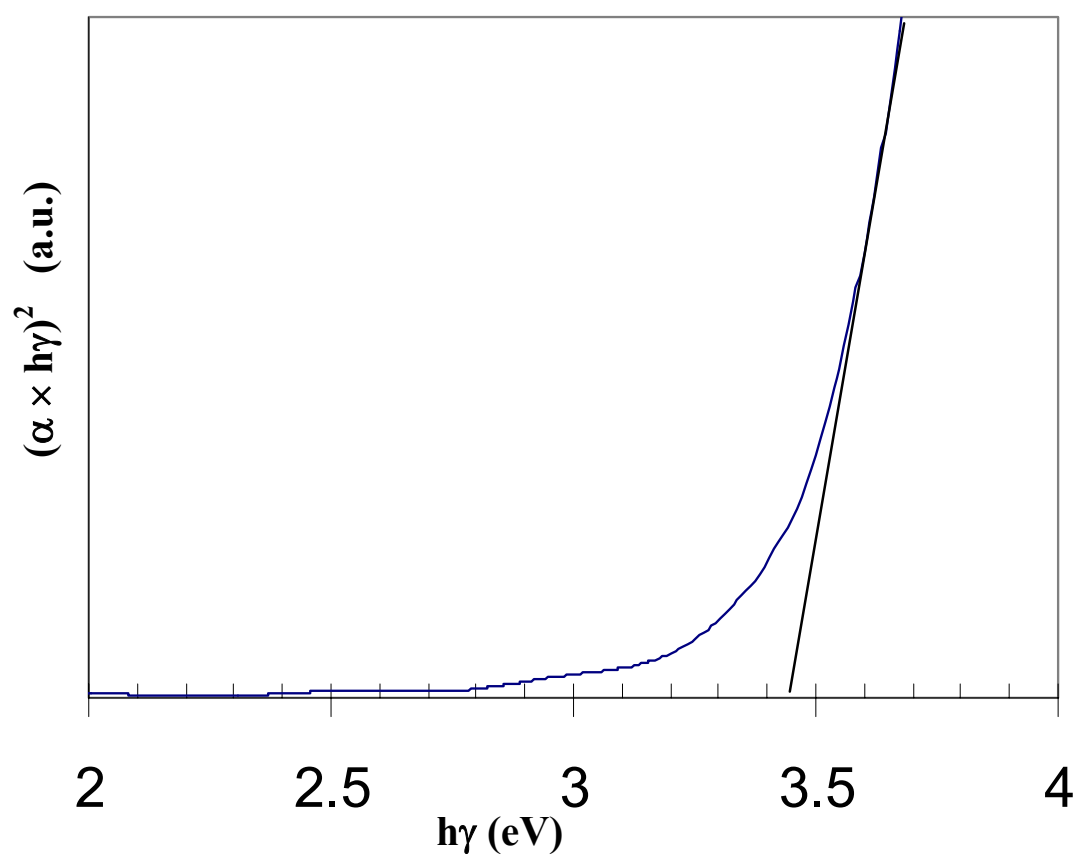

Figure A2.6 Tauc plot of the nanocrystalline GaN on quartz shows bandgap of $3.45 \mathrm{eV}$.

\section{A2.4 Summary}

Nanocrystalline gallium nitride thin film on amorphous quartz substrates had been synthesized by direct nitridation of spin-coated gallium thin film of less than $2 \mu \mathrm{m}$ under nitrogen plasma. The films have a thickness of around $0.5 \mu \mathrm{m}$ and the crystallites have an average size of around $85 \mathrm{~nm}$. Cross section TEM showed a sharp interface between GaN and quartz substrates. The gallium nitride has a polycrystalline nature and the sizes of crystallites increased with more cycles of growth. A band-gap of $3.45 \mathrm{eV}$ was determined from UV-Vis spectroscopy for the GaN films on amorphous quartz substrates. 


\section{APPENDIX III \\ PHYSICAL AND THERMODYNAMIC DATA OF GaN-N-Ga SYSTEM}

\section{A3.1 Physical Properties of Gallium}

Gallium is a group III element that was discovered by French chemist Paul-Émile Lecoq de Boisbaudran in 1875 and named from the Latin word for France, Gallia*. The most interesting properties of gallium are its low melting point $\left(\sim 30^{\circ} \mathrm{C}\right)$ and low vapor pressure $\left(8.3 \times 10^{-4}\right.$ torr at $\left.1000^{\circ} \mathrm{C}\right)$. Gallium alloys with most of the other metals and forms eutectic with much lower melting points. For example, the eutectic of Ga-In (78.6wt $\%$ of Ga, $21.4 \mathrm{wt} \% \mathrm{In}$ ) has a melting point of $15.3{ }^{\circ} \mathrm{C}$ [White, \& Okamoto, 1992]. The properties of gallium are list in Table A3.1.

The applications of gallium include coating materials for mirrors, high temperature thermometers, alloying materials for welding, source materials for solid-state semiconductors such as $\mathrm{GaAs}, \mathrm{GaN}, \mathrm{GaP}$, etc. which are widely used in electronic devices such as FETs, LEDs and Lasers.

* http://education.jlab.org/itselemental/ele031.html 
Table A3.1 Properties of gallium.

\begin{tabular}{|c|c|c|}
\hline Properties & Values \& Equations & Ref. \\
\hline Meting point & $303 \mathrm{~K}$ & $\begin{array}{l}\text { Sheka, I. A., et al., } \\
1966\end{array}$ \\
\hline Boiling point & $2676 \mathrm{~K}$ & $\begin{array}{c}\text { Sheka, I. A., et al., } \\
1966\end{array}$ \\
\hline Crystal structure & Tetragonal & $\begin{array}{c}\text { Sheka, I. A., et al., } \\
1966\end{array}$ \\
\hline Density & $\rho=6330-0.771 T\left(\mathrm{~kg} / \mathrm{m}^{3}\right)$ & $\begin{array}{c}\text { Okada \& Ozone, } \\
1992\end{array}$ \\
\hline Viscosity & $\begin{array}{l}\mu=0.01207-5.754 \times 10^{-5} T \\
+7.891 \times 10^{-8} T^{2}(\mathrm{~kg} / \mathrm{m} \cdot \mathrm{s})\end{array}$ & $\begin{array}{c}\text { Okada \& Ozone, } \\
1992\end{array}$ \\
\hline $\begin{array}{l}\text { Volumetric coefficient of } \\
\text { expansion }\end{array}$ & $\beta=-\frac{\partial \rho / \partial T}{\rho}=\frac{0.7717}{\rho}(1 / K)$ & $\begin{array}{c}\text { Okada \& Ozone, } \\
1992\end{array}$ \\
\hline Thermal conductivity & $\kappa=-7.448+0.1256 T(\mathrm{~W} / \mathrm{m} \cdot \mathrm{K})$ & $\begin{array}{l}\text { Okada \& Ozone, } \\
1992\end{array}$ \\
\hline Specific heat & $\mathrm{C}=397.6(\mathrm{~J} / \mathrm{kg} \cdot \mathrm{K})$ & $\begin{array}{l}\text { Okada \& Ozone, } \\
1992\end{array}$ \\
\hline $\begin{array}{l}\text { Electrical conductivity } \\
(298 \mathrm{~K})\end{array}$ & $6.781 \times 10^{6} /(\mathrm{Ohm} \cdot \mathrm{m})$ & $\begin{array}{c}\text { Sheka, I. A., et al., } \\
1966\end{array}$ \\
\hline Surface tension & $\begin{array}{c}\gamma=0.718 \mathrm{~N} / \mathrm{m} \\
\frac{d \gamma}{d T}=-1 \times 10^{-4} \mathrm{~N} /(\mathrm{m} \cdot \mathrm{K})\end{array}$ & \\
\hline Vapor pressure & $\begin{array}{l}\log P(\mathrm{~atm})= \\
7.37-\frac{14900}{T}-0.515 \log T\end{array}$ & $\begin{array}{l}\text { Wade \& Banister, } \\
\qquad 1975\end{array}$ \\
\hline
\end{tabular}

\section{A3.2 Thermodynamic Data Ga-N System}

$$
\begin{aligned}
& { }_{\Delta R} H_{T}^{0}={ }_{\Delta R} H_{298}^{0}+\int_{298}^{T} C_{p}^{0} d T \\
& S_{T}^{0}=S_{298}^{0}+\int_{298}^{T} C_{p}^{0} \frac{d T}{T} \\
& { }_{\Delta} G^{0}={ }_{\Delta} H^{0}-T_{\Delta} S^{0} \\
& G(P, T)=G^{o}\left(P_{0}, T\right)+\int_{P_{0}}^{P}\left(\frac{\partial G}{\partial P}\right)_{T} d P \quad \mathrm{P}_{0}=1 \mathrm{tam}
\end{aligned}
$$




$$
\begin{aligned}
& G^{o}\left(P_{0}, T\right)=H(298 K)-T \cdot \Phi(x) \quad x=\frac{T}{10000} \\
& \Phi(x)=\Psi \ln x+\sum_{k=-2}^{3} \psi_{k} x^{k} \quad \\
& H(T)=H(298 K)+T \cdot\left(\psi+\sum_{k=-2}^{3} k \psi_{k} x^{k}\right) \\
& S(T)=\psi(1+\ln x)+\sum_{k=-2}^{3}(k+1) \psi_{k} x^{k} \\
& C_{P}(T)=\psi+\sum_{k=-2}^{3} k(k+1) \psi_{k} x^{k}
\end{aligned}
$$

\begin{tabular}{|l|c|c|l|l|l|l|l|l|l|}
\hline Species & $\mathrm{T}(\mathrm{K})$ & $\begin{array}{c}\mathrm{H}(298 \mathrm{~K}) \\
\mathrm{J} / \mathrm{mol}\end{array}$ & $\psi$ & $\psi_{-2}$ & $\psi_{-1}$ & $\psi_{0}$ & $\psi_{1}$ & $\psi_{2}$ & $\psi_{3}$ \\
\hline $\mathrm{N}(\mathrm{g})$ & $\begin{array}{c}298.15- \\
3000\end{array}$ & 472680 & 20.71 & $2.073 \mathrm{E}-5$ & 0.6168 & 205.2 & 0.9006 & -2.3927 & 3.0475 \\
\hline $\mathrm{Ga}(\mathrm{l})$ & $\begin{array}{c}302.91- \\
3000\end{array}$ & 0 & 26.25 & $1.124 \mathrm{E}-3$ & 0.14777 & 126.59 & -3.3068 & 7.9605 & -4.118 \\
\hline $\mathrm{GaN}(\mathrm{s})$ & $\begin{array}{c}298.15- \\
2000\end{array}$ & -114000 & 52.86 & $-7.99 \mathrm{E}-3$ & 2.1134 & 160.26 & 1.3134 & -2.4411 & 1.9457 \\
\hline
\end{tabular}




\section{CURRICULUM VITAE}

NAME: Hongwei Li

ADDRESS: Ernst Hall, Rm 106

Department of Chemical Engineering

University of Louisville

Louisville, KY 40292

DOB: Junan County, Shandong Province, P. R. China - Jan 29, 1975

EDUCATION:

B. S., Mechanical Engineering Shandong University of Technology (now Shandong University), Jinan, China 1993-1997

M. S., Chemical Engineering Beijing Univ. of Chemical Technology, Beijing, China 1997-2000

Ph.D., Chemical Engineering Univ. of Louisville, Louisville, KY, USA 2000-2005

AWARDS:

Inducted into Who's Who Among Students in American Universities and Colleges

Awarded travel grant from ECS Electronics \& Photonics Division for 206th ECS meeting

Excellent colleges graduates (Top 5\%) in Shandong Province

Scholarships (Top 10\%) in Shandong University

PROFESSIONAL AFFILIATIONS:

Materials Research Society (MRS)

American Institute of Chemical Engineers (AIChE)

The Electrochemical Society (ECS) 


\section{PUBLICATIONS:}

1. H. Li, H. Chandrasekaran, M. K Sunkara, "Growth of Oriented Gallium Nitride Films Directly on Molten Gallium”, in preparation.

2. H. Li, M. K Sunkara, A. Chin, "Direction-dependent homo-epitaxial growth of GaN nanowires", Advanced Materials, in press, 2005.

3. H. Li, M. K. Sunkara, A. Berezin, S. I. Borenstain, "Structures and Scintillation Properties of GaN on Sapphire", submitted to Nuclear Instruments and Methods in Physics Research B.

4. H. Li, H. Chandrasekaran, M. K Sunkara, R. Collazo, Z. Sitar, M. Stukowski, K. Rajan, "Self-Oriented Growth of GaN Films on Molten Gallium”, MRS Symposium Proceedings 831, E11.34, 2004.

5. M. K. Sunkara, S. Vaddiraju, G. Bhimarasetti, H. Chandrasekaran, H. Li., R. Bogale, T. Powers, R. Thurman, "Chemical Design of Inorganic Nanowires, Nanotubes, and Nanowires Networks", in "Nanosensing: Materials and Devices", Edited by M. Saif Islam, A. K. Dutta, Proceedings of SPIE, vol. 5593, 173, Bellingham, WA, 2004.

6. H. Li, H. Chandrasekaran, M. K. Sunkara, M. Stukowski, K. Rajan, "Self Oriented Growth of GaN Films on Molten Gallium", in "State -of-the-Art Program on Compound Semiconductors XLI -and-Nitride and Wide Bandgap Semiconductors for Sensors, Photonics, and Electronics V', Edited by H. Ng and A. G. Baca, The ECS Inc., NJ, 2004.

7. S. Sharma, H. Li, H. Chandrasekaran, R. C. Mani, M. K. Sunkara, "Synthesis of Inorganic Nanowires and Nanotubes", in Encylcopedia of Nanoscience and Nanotechnology; Edited by H. Nalwa, American Scientific Publishers, Los Angeles, CA, 2003.

8. H. Li, M. K. Sunkara, "Growth of Oriented Gallium Nitride Films on Amorphous Substrates by Self-Assembly", MRS Symposium Proceedings 743, L3.11, 2003.

9. H. Li, M. K. Sunkara, "Self-oriented Growth of Gallium Nitride Films on Amorphous Substrates", $4^{\text {th }}$ Symposium on Non-Stoichiometric III-V Compounds, 105, Pacific Grove, CA, USA, 2002.

\section{PRESENTATIONS:}

1. H. Li, M. K. Sunkara, "Direct-dependent Homo-epitaxy Growth of GaN Nanowires", AIChE Annual Meeting, Cincinnati, OH, Oct. 2005. 
2. H. Li, M. K. Sunkara, H. Chandrasekaran, R. Collazo, Z. Sitar, K. Rajan, "Self-oriented GaN Film on Molten Gallium", MRS Annual Fall Meeting, Boston, MA, Dec. 2004.

3. H. Li, M. K. Sunkara, H. Chandrasekaran, S. Vaddiraju, "Self-oriented gallium nitride film growth", 8th Wide-Bandgap III-Nitride Workshop, Richmond, VA, Sep. 2003.

4. H. Li, S. Vaddiraju, H. Chandrasekaran, M. K. Sunkara, "Direct Synthesis of Gallium Nitride Nanowires and Micro Tubes", KY Nano Materials 2nd Meeting, Louisville, KY, Sep. 2003.

5. H. Li, H. Chandrasekaran, M. K. Sunkara, "Self-oriented GaN Crystal Growth”, MRS Annual Fall Meeting, Boston, MA, Dec. 2002.

6. H. Li, H. Chandrasekaran, M. K. Sunkara, "Self-oriented GaN Thin Film Growth on Amorphous and Crystalline Substrates", AIChE Annual Meeting, Indianapolis, Nov. 2002. 